\title{
Tuning Supramolecular Polymer Assembly Through Stereoelectronic Interactions
}

Will R. Henderson, Guancen Liu, Khalil A. Abboud, Ronald K. Castellano*

George \& Josephine Butler Polymer Research Laboratory, Center for Macromolecular Science \&

Engineering, Department of Chemistry, University of Florida, PO Box 117200 Gainesville, FL 326117200, USA 


\section{Table of Contents}

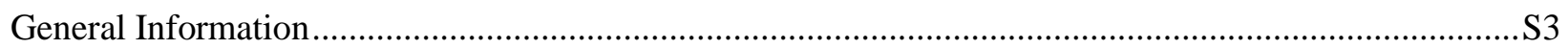

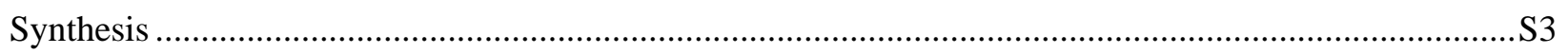

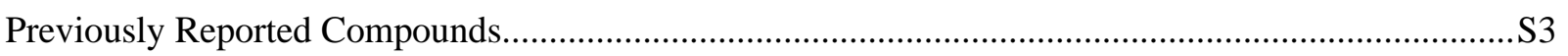

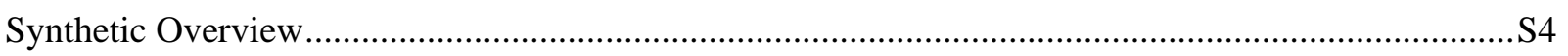

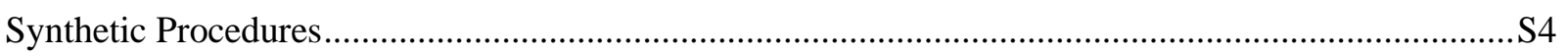

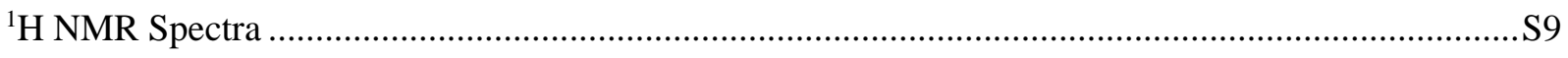

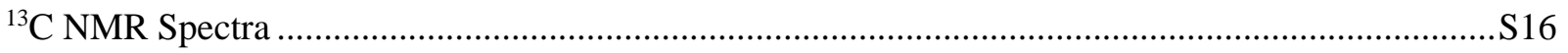

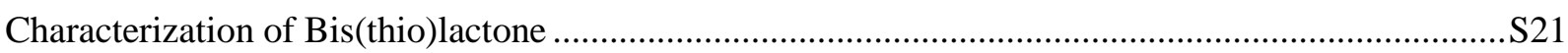

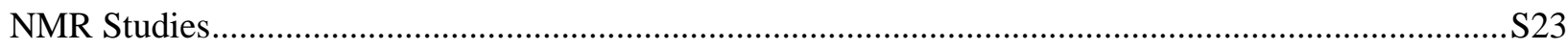

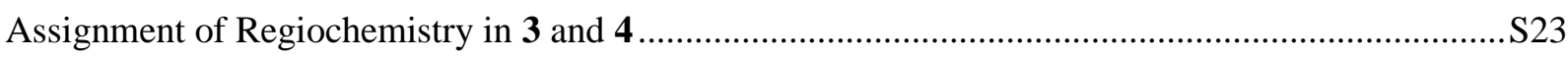

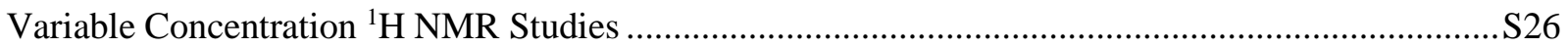

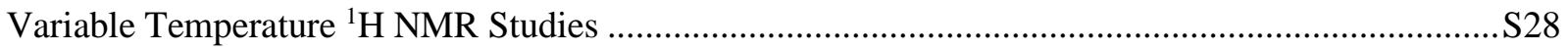

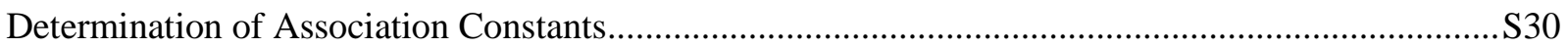

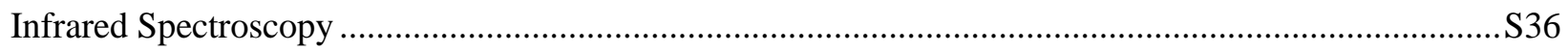

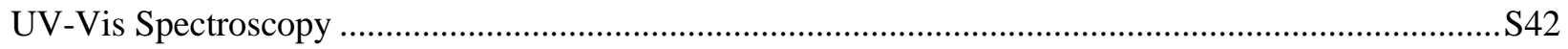

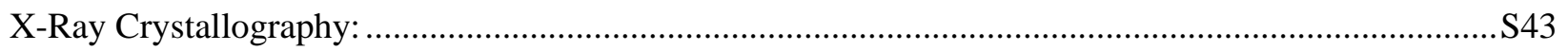

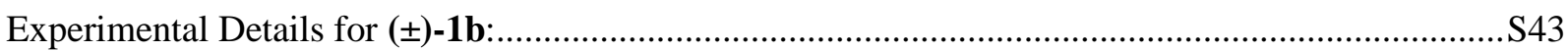

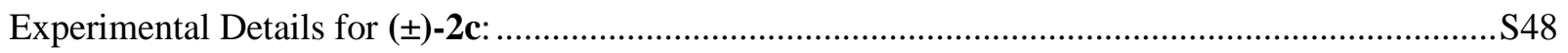

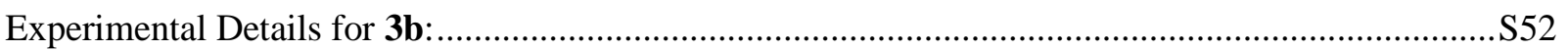

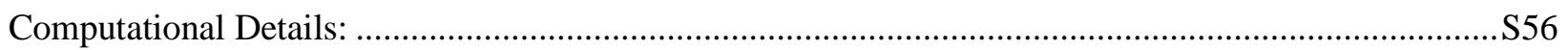

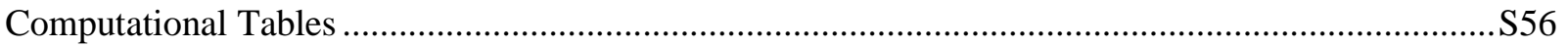

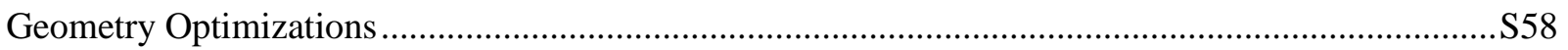

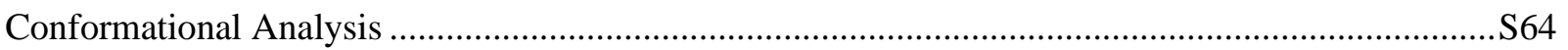

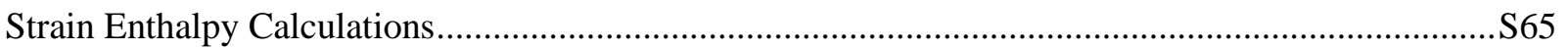

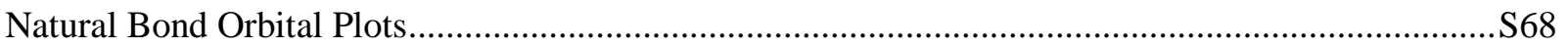

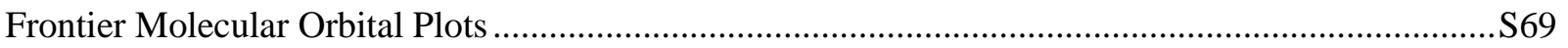

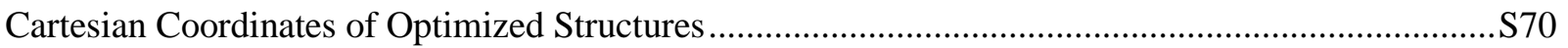

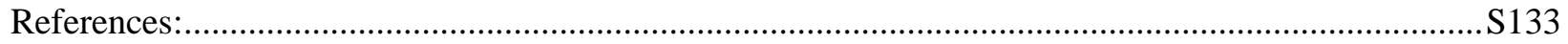




\section{General Information}

Reagents and solvents were purchased from commercial sources and used without further purification unless otherwise specified. THF, and $\mathrm{CH}_{2} \mathrm{Cl}_{2}$, were degassed in $20 \mathrm{~L}$ drums and passed through two sequential activated alumina purification columns under a positive argon atmosphere. Toluene was dried with $4 \AA$ molecular sieves and degassed by bubbling argon for 30 minutes. Thin layer chromatography (TLC) was performed on $\mathrm{SiO}_{2}-60 \mathrm{~F}_{254}$ aluminum plates with visualization by UV light or staining. Flash column chromatography was performed using silica gel technical grade, pore size $60 \AA$, 230-400 mesh particle size, $40-63 \mu \mathrm{m}$ particle size from Sigma-Aldrich. 500 (125) $\mathrm{MHz}{ }^{1} \mathrm{H}\left({ }^{13} \mathrm{C}\right) \mathrm{NMR}$ were recorded on an INOVA 500 spectrometer, and $600(150){ }^{1} \mathrm{H}\left({ }^{13} \mathrm{C}\right) \mathrm{NMR}$ were recorded on a Bruker 600 spectrometer. Chemical shifts $(\delta)$ are given in parts per million $(\mathrm{ppm})$ relative to TMS and referenced to residual protonated solvent purchased from Cambridge Isotope Laboratories, Inc. or Sigma Aldrich $\left(\mathrm{CDCl}_{3}: \delta_{\mathrm{H}} 7.26\right.$ ppm, $\delta_{\mathrm{C}} 77.16 \mathrm{ppm}$; MeOD- $d_{4}: \delta_{\mathrm{H}} 3.31 \mathrm{ppm}, \delta_{\mathrm{C}} 49.00 \mathrm{ppm}$; DMSO- $\left.d_{6}: \delta_{\mathrm{H}} 2.50 \mathrm{ppm}, \delta_{\mathrm{C}} 39.52 \mathrm{ppm}\right)$. Solvent mixtures are referenced to MeOD- $d_{4}$. Abbreviations used are s (singlet), $\mathrm{d}$ (doublet), $\mathrm{t}$ (triplet), q (quartet), quin (quintet), hp (heptet), b (broad), and m (multiplet). ESI-TOF-, and DART-TOF-MS spectra were recorded on an Agilent 6210 TOF spectrometer with MassHunter software.

\section{Synthesis}
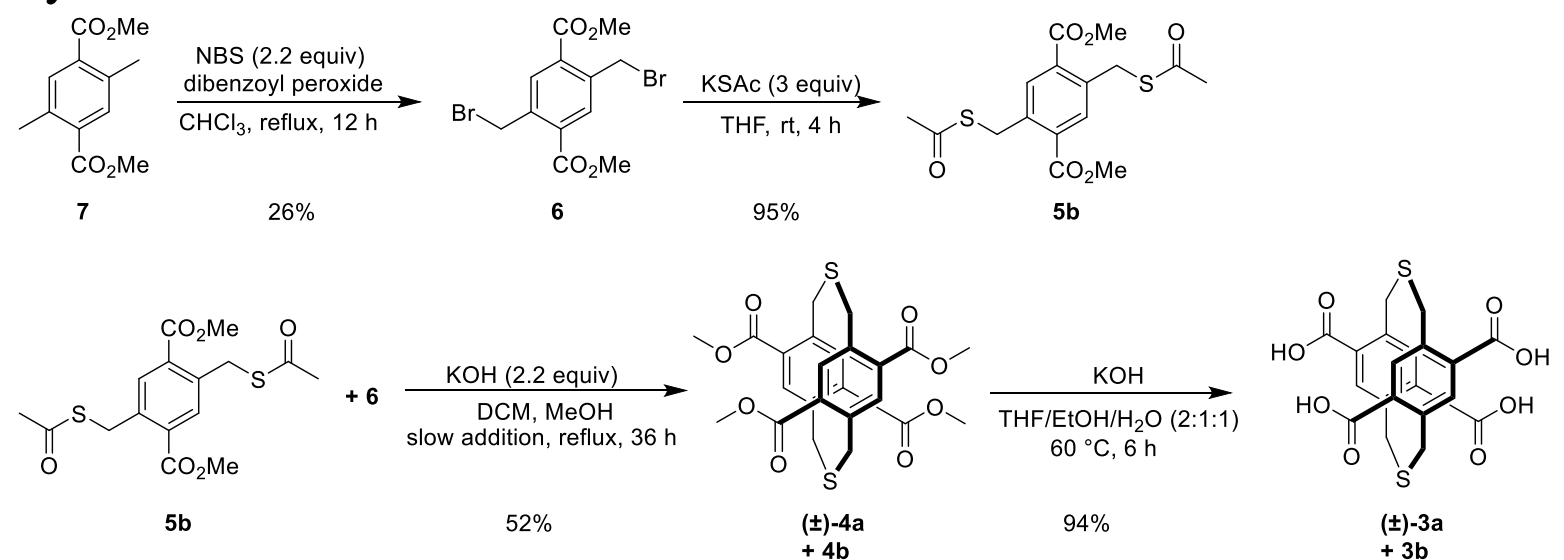

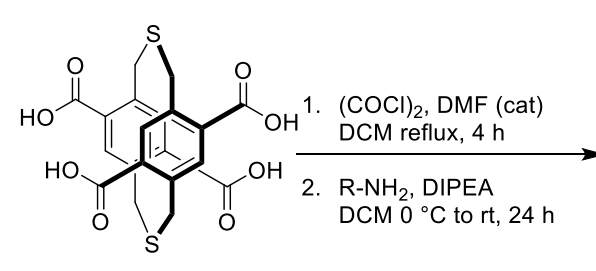

$( \pm)-3 a$
$+3 b$

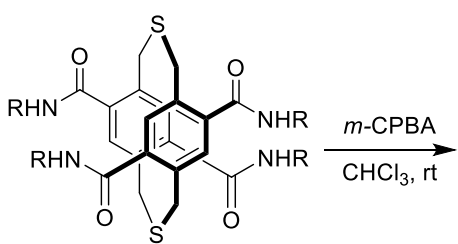

(士)-1a, R $=n$-hexyl $23 \%$ ( $\mathbf{\pm})-1 \mathbf{b}, \mathrm{R}=$ benzyl $16 \%$ (士)-1c, $\mathrm{R}=n$-propyl $17 \%$

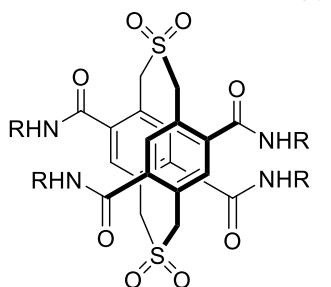

(士)-2a, $\mathrm{R}=n$-hexyl $75 \%$ (士)-2b, R = benzyl N.D.

(士)-2c, $\mathrm{R}=n$-propyl $75 \%$

Scheme S1. The optimized synthesis of dt[3.3]pCpTAs $( \pm)-1$ and $( \pm)-2$.

\section{Previously Reported Compounds}

Compounds $\mathbf{6}$ and 7 were prepared according to literature procedures. ${ }^{1-2}$ Compound 5a was prepared according to a modification of a literature procedure using TMSCHN 2 instead of $\mathrm{CH}_{2} \mathrm{~N}_{2}$ for safety reasons. $^{3}$ 


\section{Synthetic Overview}

The optimized synthesis of dithia[3.3]paracyclophane-tetracarboxamides begins with a double benzylic bromination of 2,5-dimethylterephthalate (7) to yield 1,4-bis(bromomethyl)-2,5-dimethylterephthalate (6), which can be converted to the corresponding bisthioacetate-2,5-dimethylterephthalate $(\mathbf{5 b})$ with potassium thioacetate in THF. Macrocyclization of $\mathbf{6}$ and $\mathbf{5 b}$ through slow addition of a 1:1 mixture of $\mathbf{6}$ and $\mathbf{5 b}$ in DCM to a refluxing solution of $\mathrm{KOH}$ in $\mathrm{MeOH}$ yields dithia[3.3]paracyclophane-tetraester $(( \pm)-\mathbf{4 a}$ and a mixture with the pseudo-gem isomer ( \pm )-4b). Hydrolysis of ester $( \pm)-4 a$ to acid ( \pm )-3a (along with pseudogem isomer ( \pm -3b) is accomplished with $\mathrm{KOH}$ in $\mathrm{THF} / \mathrm{H}_{2} \mathrm{O}$. Amide formation to target dt[3.3]pCpTAs $( \pm)-1$ proceeds through the acyl chloride intermediate formed with oxalyl chloride and catalytic DMF. Oxidation of the dt[3.3]pCpTAs $( \pm)-1$ with sulfide bridge to sulfone bridge ( \pm )-2 occurred smoothly with $m$-CPBA in $\mathrm{CHCl}_{3}$ at room temperature.

Synthetic Procedures<smiles>COC(=O)c1cc(CBr)c(C(=O)OC)cc1CBr</smiles>

\section{dimethyl-2,5-bis(bromomethyl)terephthalate (6)}

Compound $7^{1}$ (7.8 g, $35 \mathrm{mmol}$ ), $N$-bromosuccinimide (16 g, $87 \mathrm{mmol}$ ), and dibenzoyl peroxide (100 $\mathrm{mg}$ ) were placed in a $500 \mathrm{~mL} 3 \mathrm{~N} \mathrm{RBF}$ with a reflux condenser. Degassed $\mathrm{CHCl}_{3}(250 \mathrm{~mL})$ was added via canula and the solution was brought to reflux for 10 hours. The solution was cooled to $0{ }^{\circ} \mathrm{C}$ with an ice bath, and the resulting succinimide was removed by filtration. The solution was washed with $1 \mathrm{M} \mathrm{NaOH}$ and dried with $\mathrm{MgSO}_{4}$. The solvent was removed in vacuo and the solid was purified by successive recrystallizations from $\mathrm{CHCl}_{3}: \mathrm{EtOH}\left(\mathrm{ca} .3: 1 \mathrm{v} / \mathrm{v}\right.$ ) to yield $3.4 \mathrm{~g}$ (26\% yield) of 6 as white needles. ${ }^{1} \mathrm{H}$ NMR matched reported values. ${ }^{1}$<smiles>COC(=O)c1cc(CS)c(C(=O)OC)cc1CS</smiles>

\section{dimethyl-2,5-bis(thiomethyl)terephthalate (5a)}

Compound 6 (1.21 g, $3.18 \mathrm{mmol})$ and thiourea $(1.21 \mathrm{~g}, 15.9 \mathrm{mmol})$ were placed in a $100 \mathrm{~mL}$ RBF and 50 $\mathrm{mL}$ EtOH was added. The solution was brought to reflux for 4 hours. After 4 hours the solution was cooled to room temperature and the precipitate was collected by filtration. The solid was then redissolved in degassed $2 \mathrm{M} \mathrm{NaOH}$ solution $(20 \mathrm{~mL})$ and heated to reflux overnight. The reaction mixture was cooled to room temperature and acidified with $2 \mathrm{M} \mathrm{HCl}$. The precipitate was collected by filtration to give $470 \mathrm{mg}$, $57 \%$ yield of 2,5-bis(thiomethyl)terephthalic acid as a yellow/white solid. The 2,5bis(thiomethyl)terephthalic acid (500 mg, $1.94 \mathrm{mmol}$ ) was suspended in $25 \mathrm{~mL}$ THF and $5 \mathrm{~mL} \mathrm{MeOH}$ and stirred at room temperature. To this solution was added $\mathrm{TMSCHN}_{2}$ as a $2 \mathrm{M}$ solution in hexanes $(2.2 \mathrm{~mL}$, $4.4 \mathrm{mmol}$ ). The solution was stirred overnight before being quenched with $2 \mathrm{~mL}$ of AcOH. The reaction mixture was rotovapped onto silica gel and purified by silica gel chromatography (100\% DCM) to give 5a as a white solid (62 mg, $11 \%$ yield). ${ }^{1} \mathrm{H}$ NMR matched literature values. ${ }^{3}$ 
<smiles>COCc1cc(C(C)=O)c(CSC(C)=O)cc1C(=O)OC</smiles>

\section{dimethyl-2,5-bis((acetylthio)methyl)terephthalate (5b)}

Compound 6 (460 mg, $1.21 \mathrm{mmol}$ ) and potassium thioacetate $(415 \mathrm{mg}, 3.63 \mathrm{mmol})$ were placed in a $25 \mathrm{~mL}$ round bottom flask and dissolved in $6 \mathrm{~mL}$ of dry THF. The reaction mixture was stirred at room temperature for 4 hours. The reaction mixture was diluted with DCM $(15 \mathrm{~mL})$ and the residual salts removed by filtration. The solvent was removed in vacuo to give $\mathbf{5 b}$ as an off-white solid (427 $\mathrm{mg}, 95 \%$ ). ${ }^{1} \mathrm{H}$ NMR (500 MHz, Chloroform- $d$ ) $\delta 8.05(\mathrm{~s}, 2 \mathrm{H}), 4.44(\mathrm{~s}, 4 \mathrm{H}), 3.93(\mathrm{~s}, 6 \mathrm{H}), 2.30(\mathrm{~s}, 6 \mathrm{H}) .{ }^{13} \mathrm{C}$ NMR $(125 \mathrm{MHz}$, Chloroform- $d$ ) $\delta 195.58,166.58,139.21,134.18,132.05,52.70,31.84,30.32$. HRMS (ESI) $[\mathrm{M}+\mathrm{H}]+$ calc'd for $\mathrm{C}_{16} \mathrm{H}_{18} \mathrm{O}_{6} \mathrm{~S}_{2}: 371.0618$ found: 371.0605 .

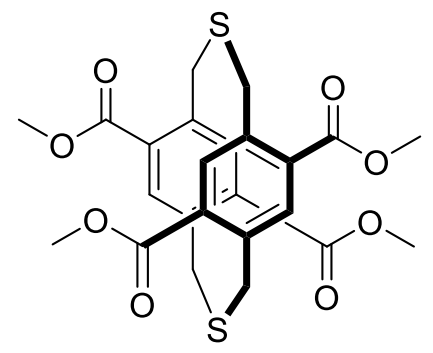

\section{2,11-dithia[3.3]paracyclophane-5,8,14,17-tetramethyl ester (( \pm$)-4)$}

Compounds 6 (1.55 g, $4.08 \mathrm{mmol})$ and $\mathbf{5 b}(1.51 \mathrm{~g}, 4.08 \mathrm{mmol})$ were dissolved in THF (200 mL) and added dropwise to a refluxing solution of $\mathrm{KOH}(978 \mathrm{mg}, 12.2 \mathrm{mmol})$ in $\mathrm{MeOH}(1 \mathrm{~L})$ over the course of 24 hours. After addition was complete the solution was heated to reflux for an additional 12 hours. The solution was cooled to room temperature and solvent removed in vacuo. The residue was purified by silica gel chromatography (DCM, $\left.R_{\mathrm{f}}=0.1\right)$ to yield $( \pm)-\mathbf{4 a}$ as a yellowish solid $(1.07 \mathrm{~g}, 52 \%$ yield) along with a percentage of the pseudo-gem isomer $4 \mathbf{b}$ (estimated as $\sim 30 \%$ from crude ${ }^{1} \mathrm{H}$ NMR). The desired product could be further purified on an analytical scale by fractional recrystallization from $\mathrm{DCM} / \mathrm{MeOH}$ to yield $( \pm)-4 a$ as colorless crystals. The regioisomeric mixture could also be used in subsequent steps and purified away from the undesired regioisomer of $( \pm)-\mathbf{1}$ as the tetra-amide. It was later found that THF could be exchanged for DCM with no noticeable changes in yield.

(士)-4a: ${ }^{1} \mathrm{H}$ NMR (500 MHz, Chloroform- $d$ ) $\delta 7.55$ (s, 4H), 4.72 (d, J = $\left.14.7 \mathrm{~Hz}, 4 \mathrm{H}\right), 3.96$ (s, 12H), 3.68 $(\mathrm{d}, \mathrm{J}=14.7 \mathrm{~Hz}, 4 \mathrm{H}) .{ }^{13} \mathrm{C}$ NMR $(125 \mathrm{MHz}$, Chloroform- $d$ ) $\delta 167.30,138.11,133.44,132.49,52.46,34.84$. HRMS (ESI) $[\mathrm{M}+\mathrm{H}]^{+}$calc'd for $\mathrm{C}_{24} \mathrm{H}_{25} \mathrm{O}_{8} \mathrm{~S}_{2}: 505.0985$ found: 505.0971.

4b: ${ }^{1} \mathrm{H}$ NMR (500 MHz, Chloroform- $d$ ) $\delta 7.62$ (s, 4H), 4.77 (d, $\left.J=15.3 \mathrm{~Hz}, 4 \mathrm{H}\right), 3.89$ (s, 12H), $3.63(\mathrm{~d}, J$ $=15.3 \mathrm{~Hz}, 4 \mathrm{H})$. 
<smiles>O=C(O)c1cc2c(C(=O)O)c(C(=O)O)c1CSC2</smiles>

2,11-dithia[3.3]paracyclophane-5,8,14,17-tetracarboxylic acid (( \pm )-3a) A mixture of compounds ( \pm )-4a and $4 \mathrm{~b}(1.07 \mathrm{~g}, 2.99 \mathrm{mmol})$ and $\mathrm{KOH}(6.8 \mathrm{~g}, 60 \mathrm{mmol})$ were heated to $60{ }^{\circ} \mathrm{C}$ in a mixture of $\mathrm{THF}(20 \mathrm{~mL})$, EtOH $(10 \mathrm{~mL})$, and $\mathrm{H}_{2} \mathrm{O}(10 \mathrm{~mL})$. After 6 hours, the solution was cooled to room temperature, acidified with $2 \mathrm{M} \mathrm{HCl}$, and extracted with EtOAc $(3 \times 50 \mathrm{~mL})$. The organic layer was dried with $\mathrm{Na}_{2} \mathrm{SO}_{4}$ and the solvent removed in vacuo to yield a mixture of ( \pm )-3a and $\mathbf{3 b}$ as an off-white solid (894 $\mathrm{mg}, 94 \%$ yield).

A pure sample of compound $\mathbf{3 b}$ could be obtained by slow diffusion of $\mathrm{H}_{2} \mathrm{O}$ into a mixture of ( \pm )-3a and 3b in DMSO. ${ }^{1} \mathrm{H}$ NMR (600 MHz, DMSO- $\left.d_{6}\right) \delta 12.80$ (br s, 4H), 7.59 (s, 4H), 4.70 (d, $\left.J=14.8 \mathrm{~Hz}, 4 \mathrm{H}\right)$, $3.72(\mathrm{~d}, J=14.8 \mathrm{~Hz}, 4 \mathrm{H}) .{ }^{13} \mathrm{C}$ NMR $\left(150 \mathrm{MHz}\right.$, DMSO- $\left.d_{6}\right) \delta 167.24,137.97,133.21,132.15,34.02$. HRMS (ESI) $[\mathrm{M}-\mathrm{H}]^{-}$calc'd for $\mathrm{C}_{20} \mathrm{H}_{15} \mathrm{O}_{8} \mathrm{~S}_{2}: 447.0214$ found 447.0230

Pure ( \pm )-3a could be obtained by hydrolysis of an analytically pure sample of $( \pm)$-4a purified by fractional crystallization following the procedure above: ${ }^{1} \mathrm{H}$ NMR (600 MHz, DMSO) $\delta 12.98$ (br s, 4H), 7.52 (s, 4H), $4.67(\mathrm{~d}, J=14.5 \mathrm{~Hz}, 4 \mathrm{H}), 3.70(\mathrm{~d}, J=14.5 \mathrm{~Hz}, 4 \mathrm{H}) .{ }^{13} \mathrm{C}$ NMR $\left(150 \mathrm{MHz}, \mathrm{DMSO}-d_{6}\right) \delta 167.71,137.53$, 133.04, 132.49, 33.98. HRMS (ESI) $[\mathrm{M}+\mathrm{Na}]^{+}$calc'd for $\mathrm{C}_{20} \mathrm{H}_{16} \mathrm{O}_{8} \mathrm{~S}_{2} \mathrm{Na}: 471.0179$ found: 471.0190 .

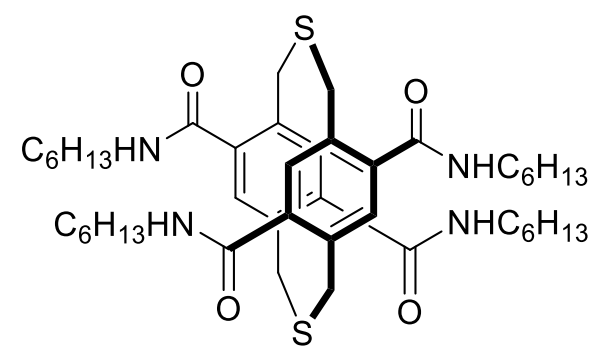

\section{2,11-dithia[3.3]paracyclophane-5,8,14,17-tetra(n-hexyl)carboxamide (( \pm )-1a)}

A mixture of compounds ( \pm )-3a and ( \pm )-3b $(237 \mathrm{mg}, 0.528 \mathrm{mmol})$ was placed in a $50 \mathrm{~mL}$ flame-dried 3neck round bottom flask with a magnetic stir bar, equipped with a reflux condenser. The solid was suspended in DCM (10 mL). Oxalyl chloride $(1.0 \mathrm{~mL}, 12 \mathrm{mmol})$ was added along with three drops of DMF. The solution was brought to reflux for 4 hours. The solvent and residual reagent were removed in vacuo and the reaction mixture redissolved in DCM $(10 \mathrm{~mL})$. The solution was cooled to $0{ }^{\circ} \mathrm{C}$ in an ice bath and hexylamine $(2.0 \mathrm{~mL}, 15 \mathrm{mmol})$ and triethylamine $(2.0 \mathrm{~mL}, 14 \mathrm{mmol})$ were added by syringe. The reaction mixture was warmed to room temperature and stirred for 12 hours. The reaction mixture was diluted with DCM $(20 \mathrm{~mL})$ and washed with $2 \mathrm{M} \mathrm{HCl}(20 \mathrm{~mL})$. The organic layer was dried with $\mathrm{MgSO}_{4}$ and the solvent was removed in vacuo. The product was purified by silica gel chromatography (20-30\% EtOAc in hexanes) to yield ( \pm )-1a as a sticky white solid (96 mg, 23\% yield). The impure fractions could be further purified by crystallization from EtOAc. ${ }^{1} \mathrm{H}$ NMR $(500 \mathrm{MHz}$, Chloroform- $d) \delta 8.40$ (br s, 4H), $6.81(\mathrm{~s}, 4 \mathrm{H}), 4.03$ (d, $\mathrm{J}=15.2 \mathrm{~Hz}, 4 \mathrm{H}), 3.47-3.18(\mathrm{~m}, 12 \mathrm{H}), 1.65(\mathrm{p}, 8 \mathrm{H}), 1.35(\mathrm{~m}, \mathrm{~J}=7.5,3.7 \mathrm{~Hz}, 24 \mathrm{H}), 0.91(\mathrm{t}, 12 \mathrm{H}){ }^{13} \mathrm{C} \mathrm{NMR}$ $\left(125 \mathrm{MHz}\right.$, Chloroform- $d /$ Methanol- $\left.d_{4}\right) \delta 169.86,136.92,134.79,129.56,40.16,35.71,31.49,29.06,26.85$, 22.56, 13.91. HRMS (ESI) $[\mathrm{M}+\mathrm{H}]^{+}$calc'd for $\mathrm{C}_{44} \mathrm{H}_{69} \mathrm{~N}_{4} \mathrm{O}_{4} \mathrm{~S}_{2}: 781.4755$ found: 781.4729 . 
<smiles>CCSCc1c(C(=O)NCc2ccccc2)cc(C(N)=O)c(C(=O)NCc2ccccc2)c1C(=O)NCc1ccccc1</smiles>

\section{2,11-dithia[3.3]paracyclophane-5,8,14,17-tetrabenzylcarboxamide (( \pm$)$-1b)}

This compound was synthesized in the same way as ( \pm )-1a with $( \pm)-\mathbf{3}(68 \mathrm{mg}, 0.15 \mathrm{mmol})$, oxalyl chloride $(0.6 \mathrm{~mL}, 7 \mathrm{mmol})$, benzylamine $(2.0 \mathrm{~mL}, 18 \mathrm{mmol})$, and triethylamine $(0.6 \mathrm{~mL}, 4 \mathrm{mmol})$. The product was further purified by silica gel chromatography (30-50\% EtOAc in hexanes) to yield ( \pm )-1b as a white solid (19 mg, 16\% yield). Slow evaporation from EtOH/EtOAc yielded single crystals suitable for X-ray diffraction. ${ }^{1} \mathrm{H}$ NMR (500 MHz, Chloroform- $d$ ) $\delta 8.33$ (br s, 4H), $7.47-7.23$ (m, 20H), 6.87 (br s, 4H), 4.56 $(\mathrm{dd}, J=14.3,6.0 \mathrm{~Hz}, 4 \mathrm{H}), 4.46(\mathrm{dd}, J=14.3,5.2 \mathrm{~Hz}, 4 \mathrm{H}), 3.84(\mathrm{~d}, J=15.3 \mathrm{~Hz}, 4 \mathrm{H}), 3.11(\mathrm{~d}, J=15.4 \mathrm{~Hz}$, $4 \mathrm{H}) .{ }^{13} \mathrm{C}$ NMR (125 MHz, Chloroform- $d$ ) $\delta 169.1,138.5,136.6,134.9,128.8,128.8,128.5,127.6,44.2$, 35.6. HRMS (ESI) $[\mathrm{M}+\mathrm{H}]^{+}$calc'd for $\mathrm{C}_{48} \mathrm{H}_{45} \mathrm{~N}_{4} \mathrm{O}_{4} \mathrm{~S}_{2}: 805.2877$ found: 805.2838.

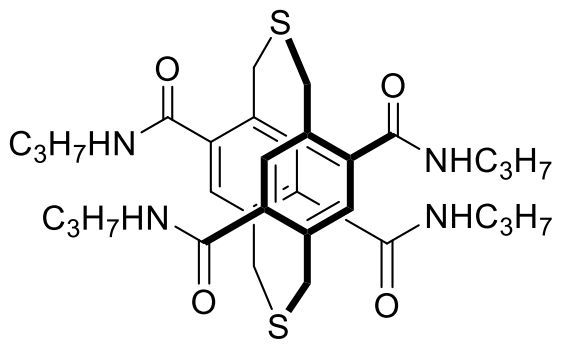

\section{2,11-dithia[3.3]paracyclophane-5,8,14,17-tetra(n-propyl)carboxamide (( \pm$)$-1c)}

This compound was synthesized in the same way as $( \pm)-\mathbf{1 a}$ with $( \pm)-3(257 \mathrm{mg}, 0.573 \mathrm{mmol})$, oxalyl chloride $(1.0 \mathrm{~mL}, 12 \mathrm{mmol})$, propylamine $(2.0 \mathrm{~mL}, 24 \mathrm{mmol})$, and triethylamine $(1.0 \mathrm{~mL}, 7.1 \mathrm{mmol})$. The product was purified by silica gel chromatography (50\% EtOAc in Hexanes) to yield ( \pm )-1c as a white powder (58 mg, $17 \%$ yield). ${ }^{1} \mathrm{H}$ NMR $\left(600 \mathrm{MHz}\right.$, Methanol- $\left.d_{4}\right) \delta 7.23(\mathrm{~s}, 4 \mathrm{H}), 4.23(\mathrm{~d}, J=15.3 \mathrm{~Hz}, 4 \mathrm{H})$, $3.67(\mathrm{~d}, J=15.3 \mathrm{~Hz}, 4 \mathrm{H}), 3.40$ (hept, $J=6.6,6.2 \mathrm{~Hz}, 8 \mathrm{H}), 1.74$ (qd, $J=7.3,1.5 \mathrm{~Hz}, 8 \mathrm{H}), 1.06$ (t, $J=7.4$ $\mathrm{Hz}, 12 \mathrm{H}) .{ }^{13} \mathrm{C} \mathrm{NMR}\left(150 \mathrm{MHz}\right.$, Methanol- $\left.d_{4}\right) \delta 171.47,138.46,136.65,130.99,42.83,36.69,23.54,12.15$. HRMS (ESI) $[\mathrm{M}+\mathrm{H}]^{+}$calc'd for $\mathrm{C}_{32} \mathrm{H}_{45} \mathrm{~N}_{4} \mathrm{O}_{4} \mathrm{~S}_{2}: 613.2877$ found: 613.2864 .

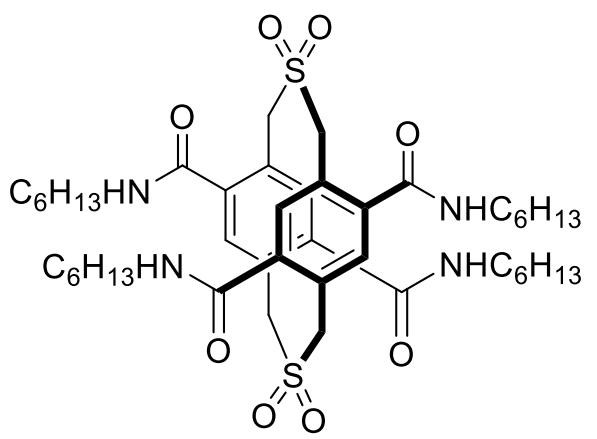

2,11-dithia[3.3]paracyclophane-5,8,14,17-tetra(n-hexyl)carboxamide tetraoxide (( \pm$)$-2a) 
Compound ( \pm -1a $(19 \mathrm{mg}, 0.025 \mathrm{mmol})$ was placed in a vial with $m$-CPBA (43 $\mathrm{mg}, 0.25 \mathrm{mmol})$ and dissolved in $2 \mathrm{~mL} \mathrm{CHCl}_{3}$. The reaction mixture was stirred at room temperature for 3 days, quenched with $\mathrm{NaHSO}_{3}$, and purified by silica gel chromatography (20-50\% EtOAc in hexanes) to yield ( \pm )-2a as a white solid (16 mg, 75\% yield). Further purification could be performed by recrystallization from EtOAc.

${ }^{1} \mathrm{H}$ NMR $(500 \mathrm{MHz}$, Chloroform- $d$ ) $\delta 7.87$ (br s, 4H), $7.52(\mathrm{~s}, 4 \mathrm{H}), 4.79(\mathrm{~d}, J=14.6 \mathrm{~Hz}, 4 \mathrm{H}), 3.99(\mathrm{~d}, J=$ $14.8 \mathrm{~Hz}, 4 \mathrm{H}), 3.40-3.21(\mathrm{~m}, 8 \mathrm{H}), 1.58(\mathrm{q}, J=7.3 \mathrm{~Hz}, 8 \mathrm{H}), 1.46-1.16(\mathrm{~m}, 24 \mathrm{H}), 0.90(\mathrm{t}, J=7.2 \mathrm{~Hz}, 12 \mathrm{H})$.

${ }^{13} \mathrm{C}$ NMR (125 MHz, Chloroform- $d$ ) $\delta$ 168.2, 137.8, 131.0, 128.0, 58.5, 40.6, 31.7, 29.2, 27.0, 22.8, 14.2. HRMS (ESI) $[\mathrm{M}+\mathrm{H}]^{+}$calc'd for $\mathrm{C}_{44} \mathrm{H}_{68} \mathrm{~N}_{4} \mathrm{O}_{8} \mathrm{~S}_{2}: 845.4551$ found: 845.4585 .

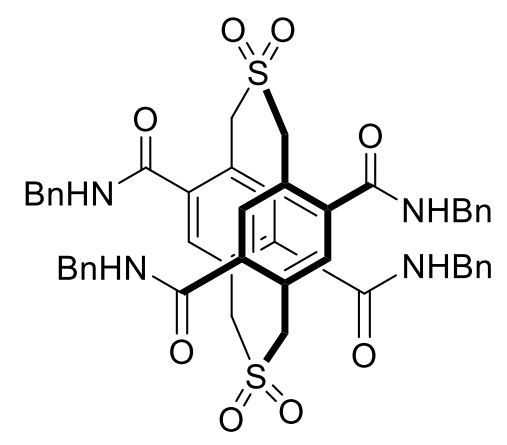

\section{2,11-dithia[3.3] paracyclophane-5,8,14,17-tetrabenzylcarboxamide tetraoxide (( \pm$)-2 b)$}

Compound ( \pm )-2b could be synthesized in the same way as $( \pm)-\mathbf{2 a}$, but full characterization could not be accomplished due to significant insolubility. ${ }^{1} \mathrm{H}$ NMR $\left(600 \mathrm{MHz}\right.$, Methanol- $\left.d_{4} / \mathrm{CDCl}_{3}\right) \delta 7.73(\mathrm{~s}, 4 \mathrm{H}), 7.40$ $(\mathrm{d}, J=7.5 \mathrm{~Hz}, 8 \mathrm{H}), 7.34(\mathrm{t}, J=7.6 \mathrm{~Hz}, 8 \mathrm{H}), 7.26(\mathrm{t}, J=7.4 \mathrm{~Hz}, 4 \mathrm{H}), 4.99(\mathrm{~d}, J=14.9 \mathrm{~Hz}, 4 \mathrm{H}), 4.51(\mathrm{td}, J$ $=14.9,3.5 \mathrm{~Hz}, 8 \mathrm{H}), 4.32(\mathrm{~d}, J=14.8 \mathrm{~Hz}, 5 \mathrm{H})$. HRMS (DART) $[\mathrm{M}+\mathrm{H}]^{+}$calc'd for $\mathrm{C}_{48} \mathrm{H}_{45} \mathrm{~N}_{4} \mathrm{O}_{8} \mathrm{~S}_{2}: 869.2673$ found: 869.2704 .

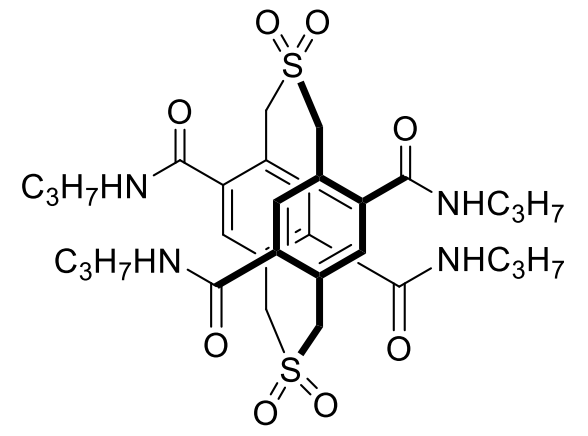

\section{2,11-dithia[3.3]paracyclophane-5,8,14,17-tetra(n-propyl)carboxamide tetraoxide (( \pm$)-2 c)$}

This compound was synthesized in the same way as ( \pm )-2a with ( \pm )-1c (28 mg, $0.046 \mathrm{mmol})$, and $m$-CPBA (47 mg, $0.27 \mathrm{mmol}$ ). The product was purified by silica gel chromatography (70\% EtOAc in Hexanes) and was further purified by recrystallization from methanol. ${ }^{1} \mathrm{H}$ NMR $\left(600 \mathrm{MHz}\right.$, Methanol- $\left.d_{4}\right) \delta 7.73(\mathrm{~s}, 4 \mathrm{H})$, 4.98 (br s, 4H), $3.44-3.34(\mathrm{~m}, 8 \mathrm{H}), 1.73$ (qd, $J=7.3,1.3 \mathrm{~Hz}, 8 \mathrm{H}), 1.05(\mathrm{t}, J=7.4 \mathrm{~Hz}, 12 \mathrm{H})$ One benzylic doublet suspected to be overlapping with solvent. ${ }^{13} \mathrm{C}$ NMR (150 MHz, Methanol- $\left.d_{4}\right) \delta 170.32$, 139.22, 132.87, 129.64, 58.30, 43.02, 23.43, 12.08. HRMS (ESI) $[\mathrm{M}+\mathrm{H}]^{+}$calc'd for $\mathrm{C}_{32} \mathrm{H}_{45} \mathrm{~N}_{4} \mathrm{O}_{8} \mathrm{~S}_{2}: 677.2673$ found: 677.2681 . 

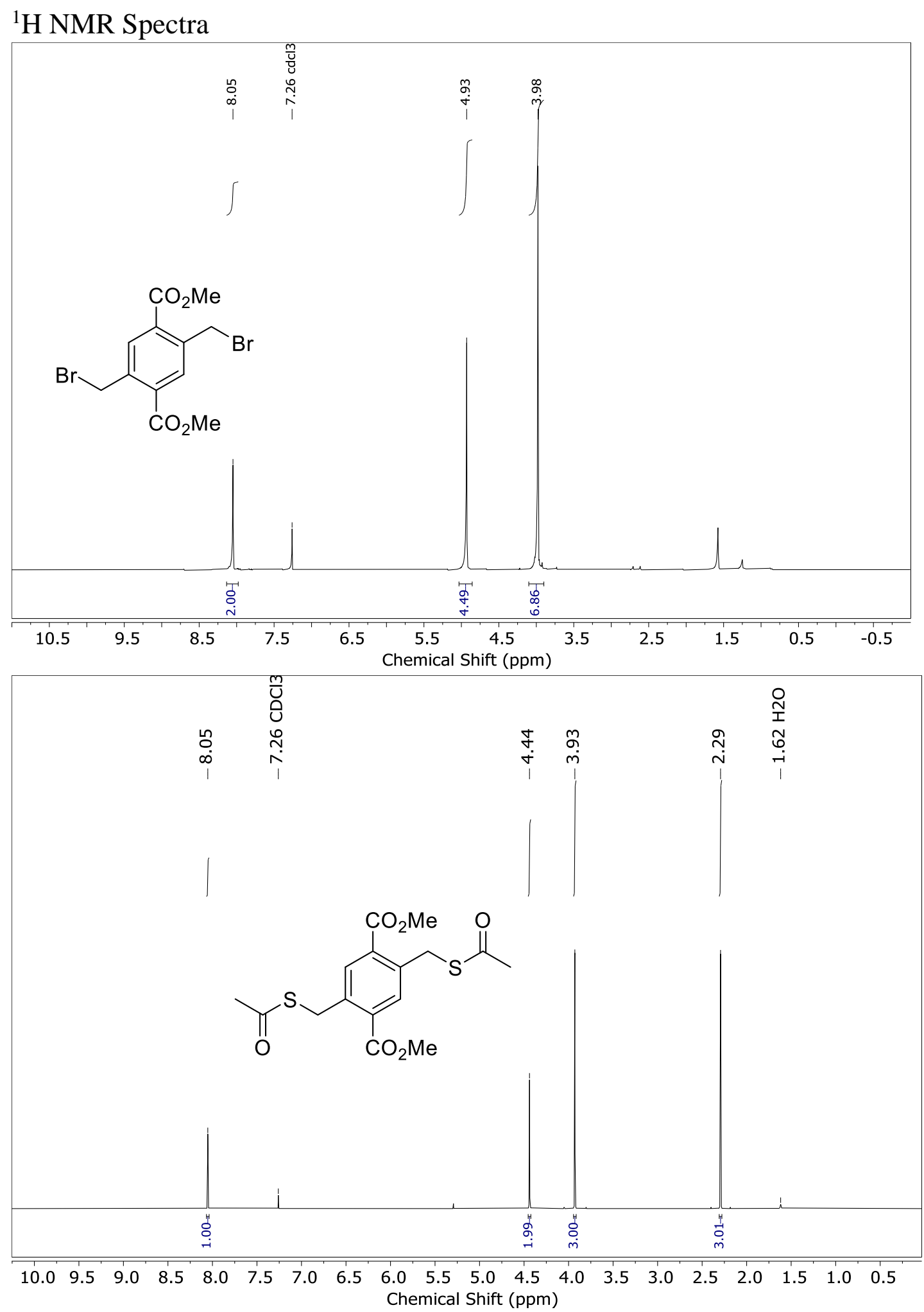

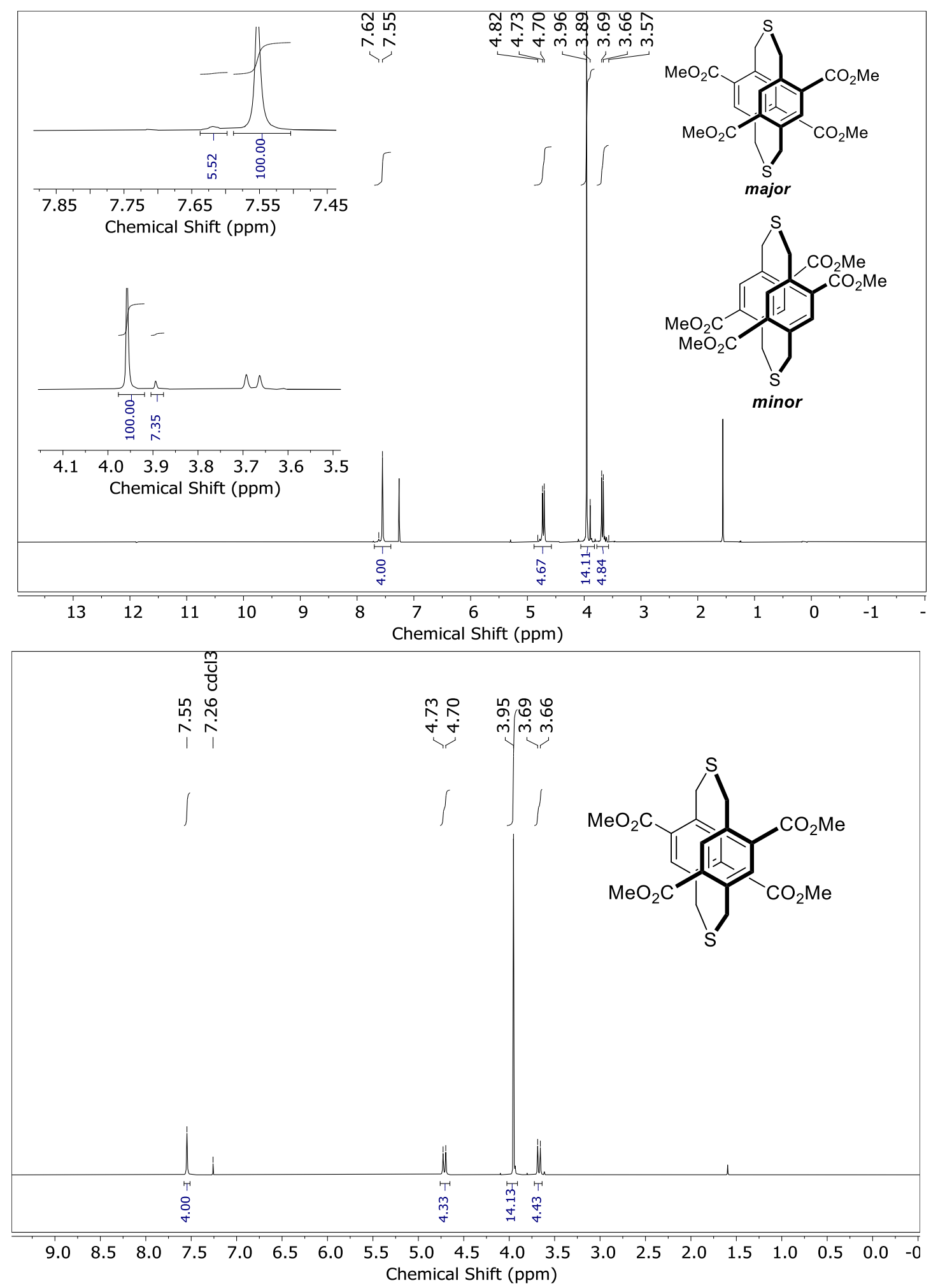

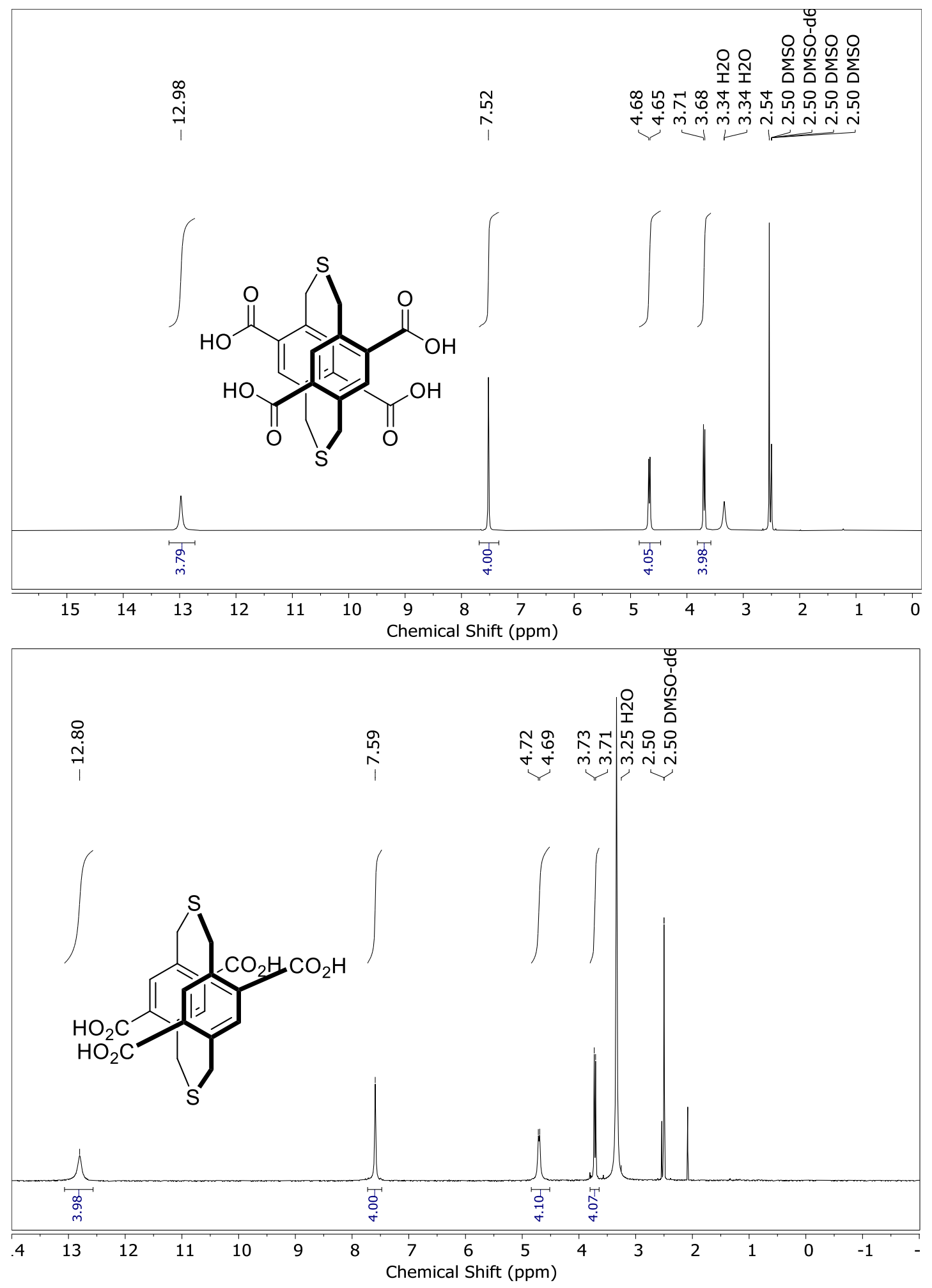

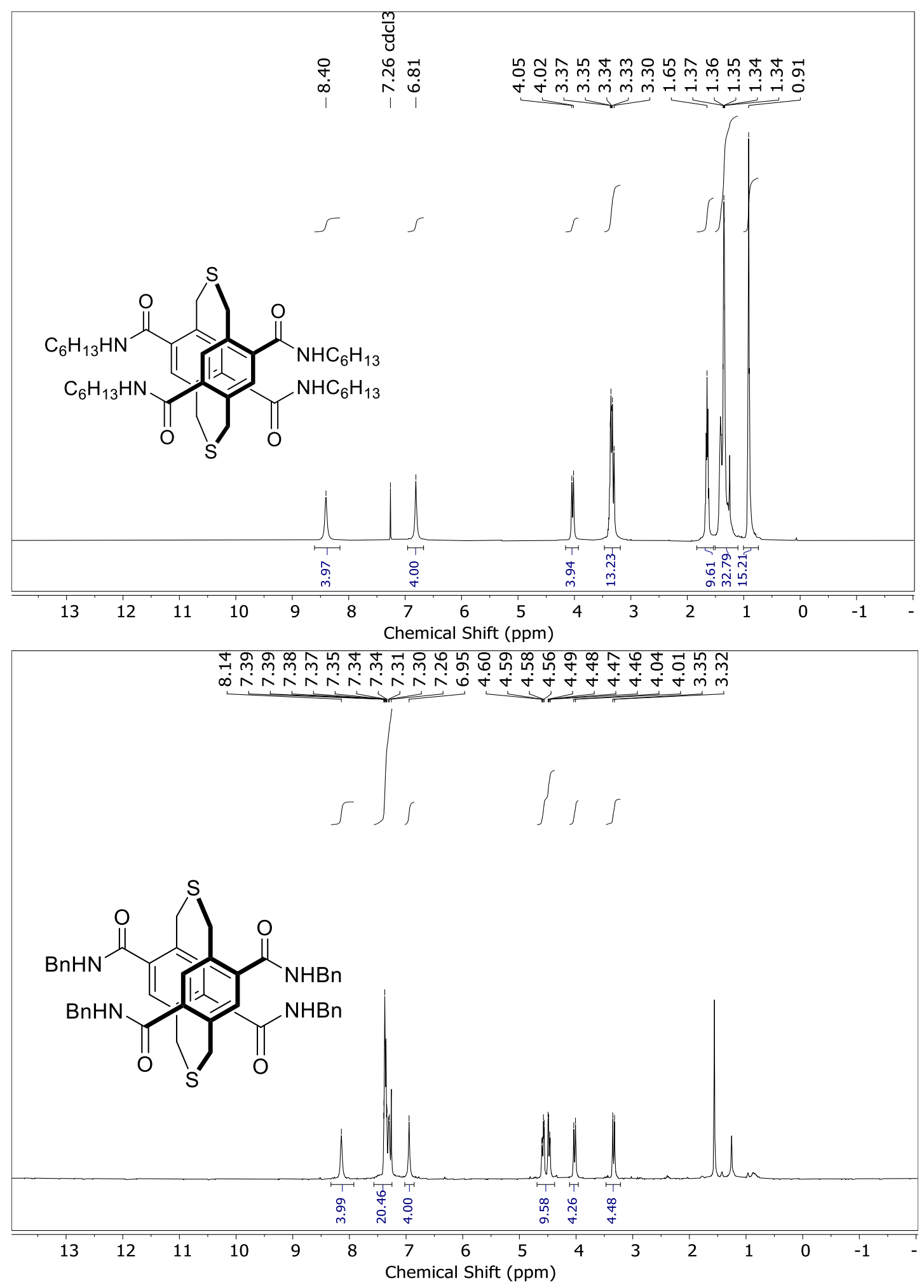

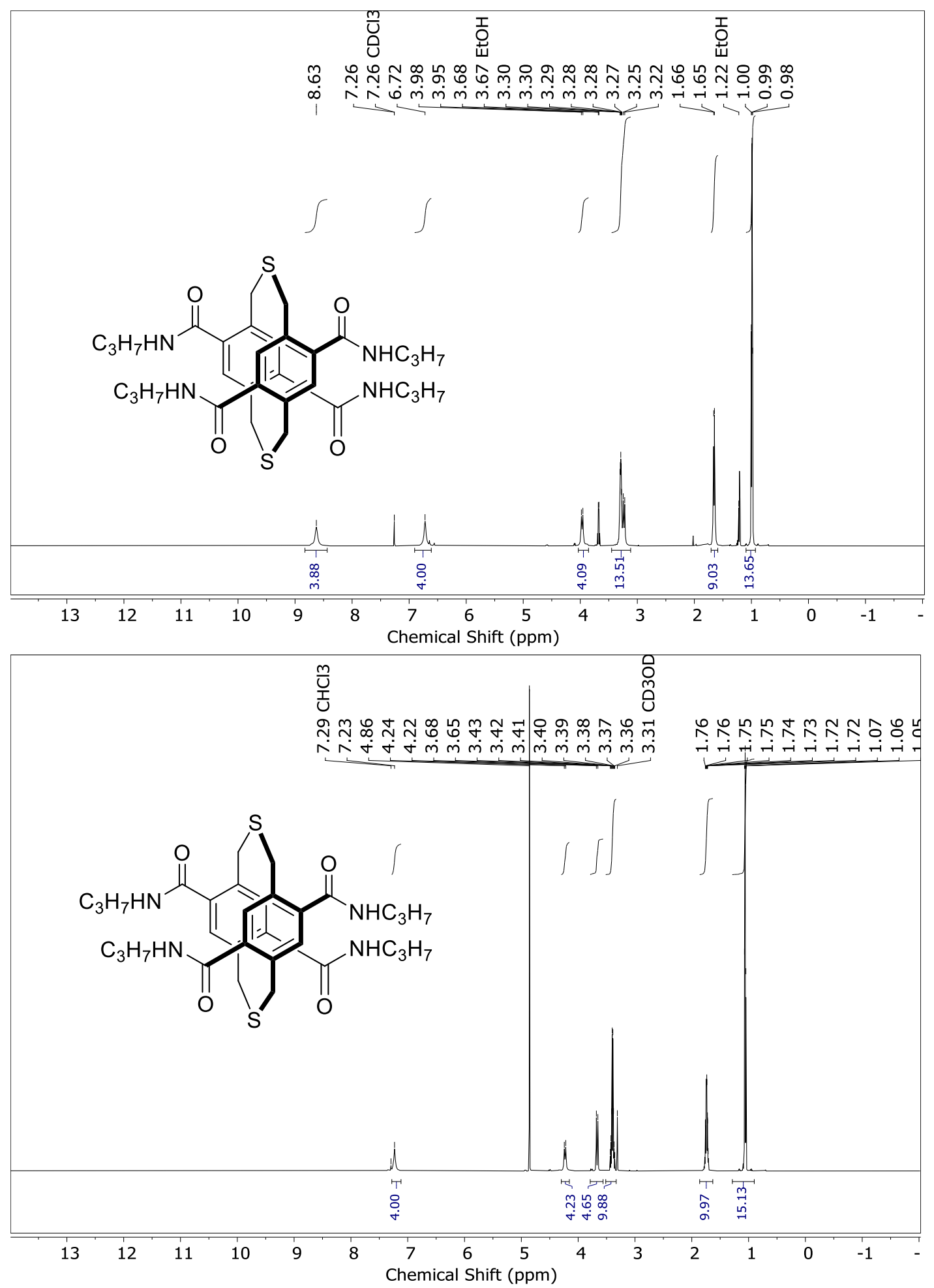

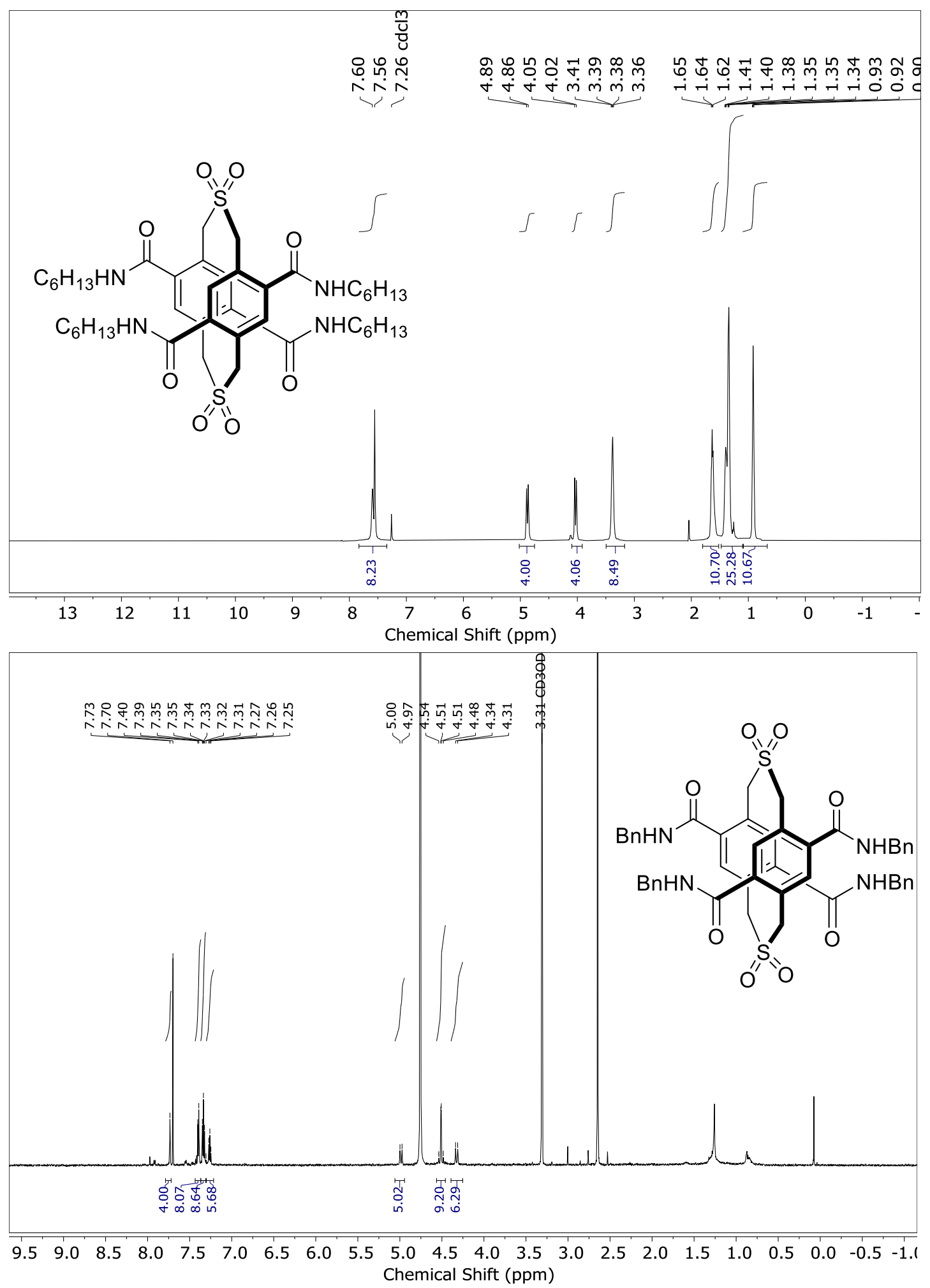


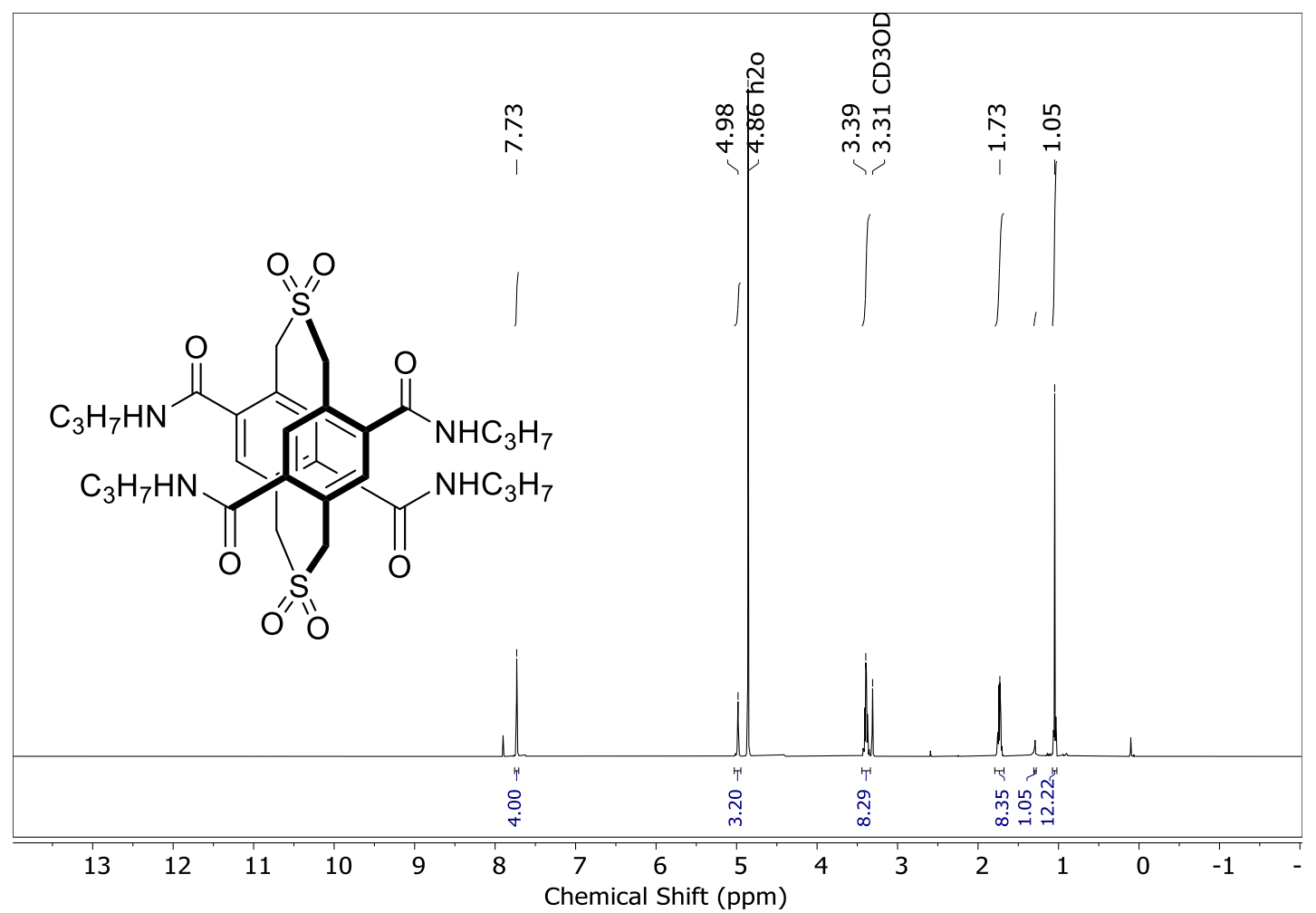




\section{${ }^{13} \mathrm{C}$ NMR Spectra}
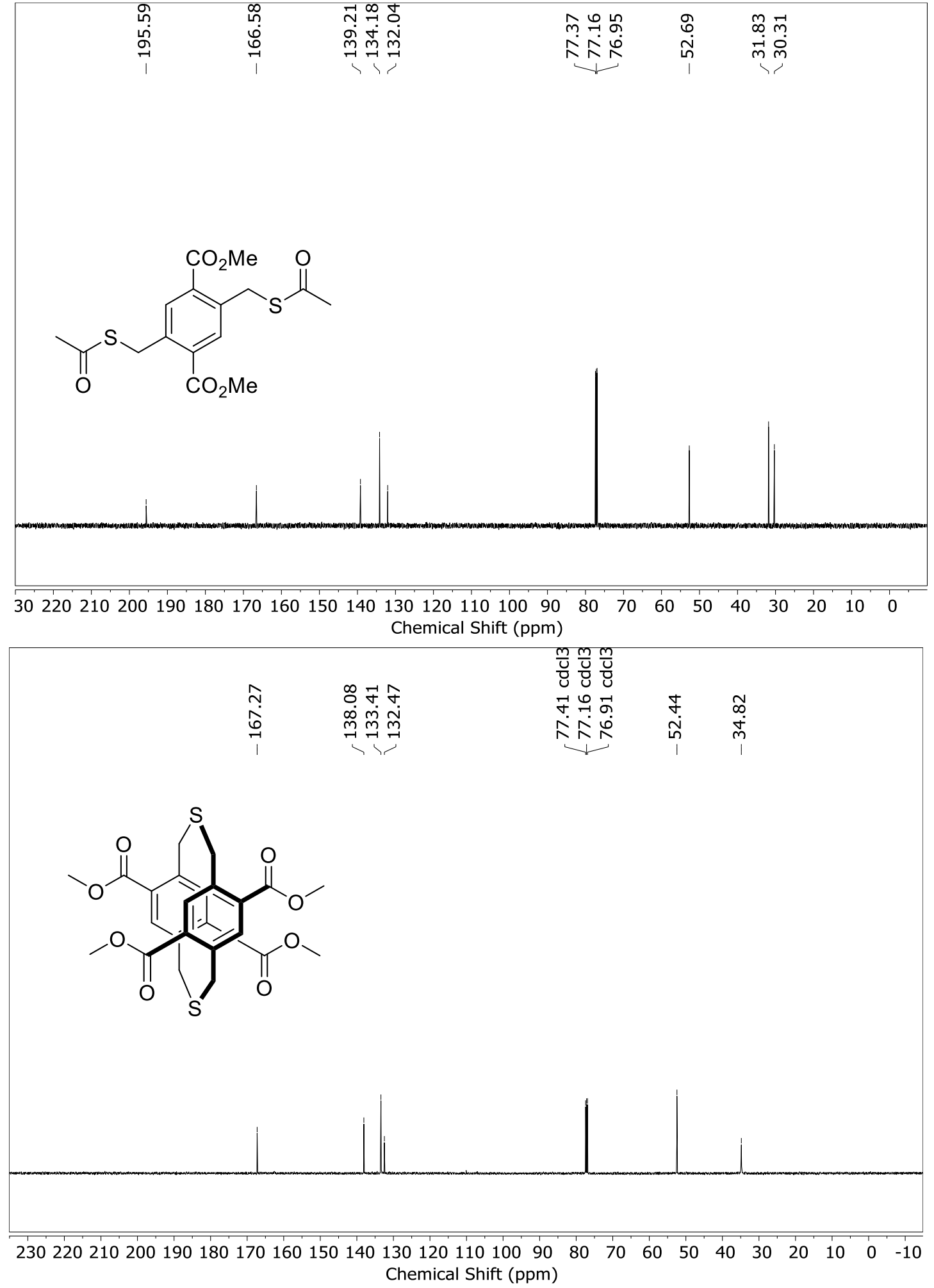


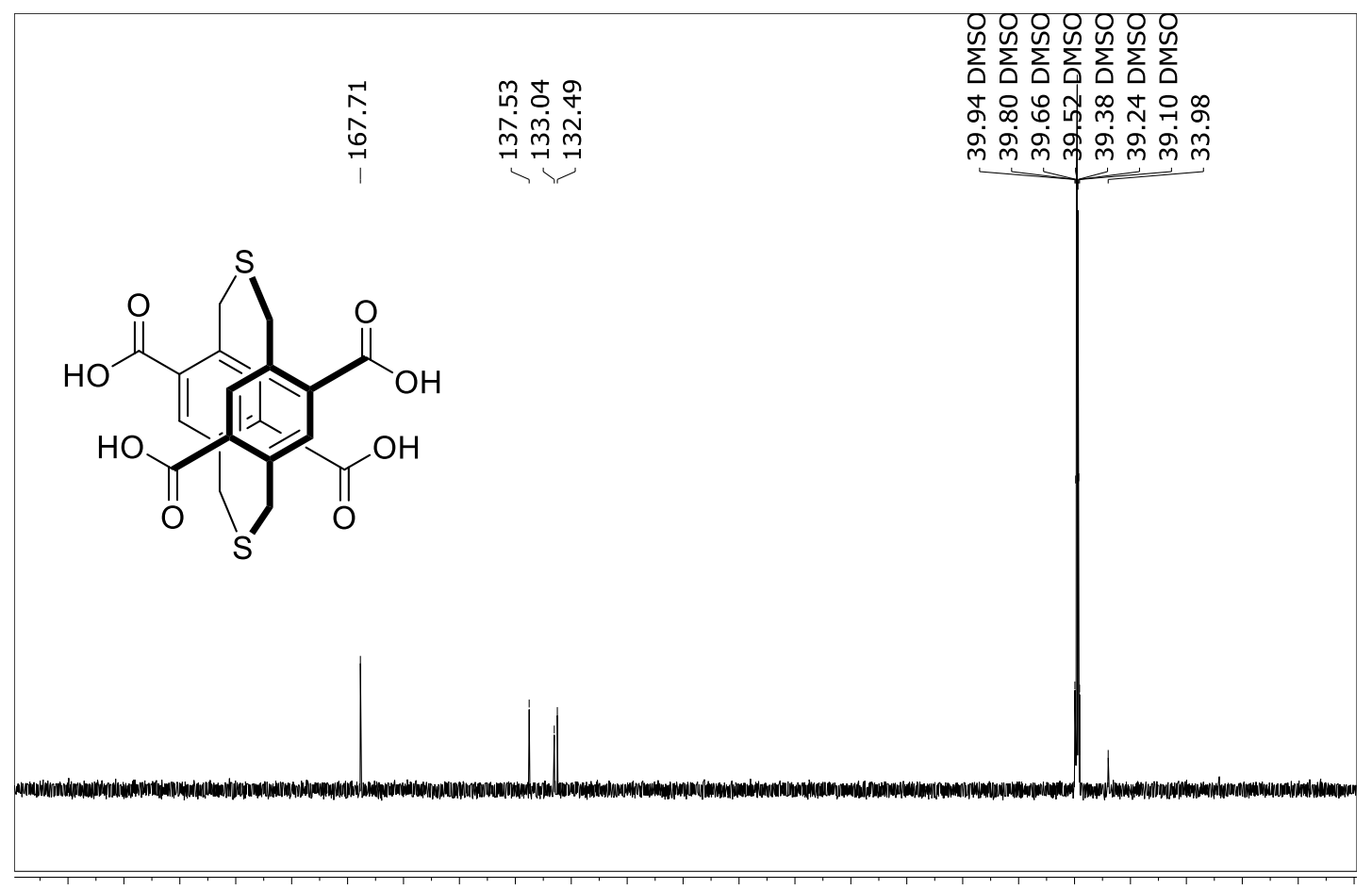

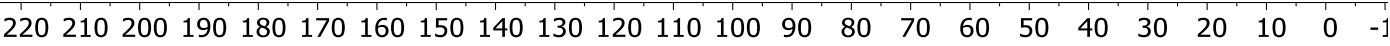
Chemical Shift (ppm)

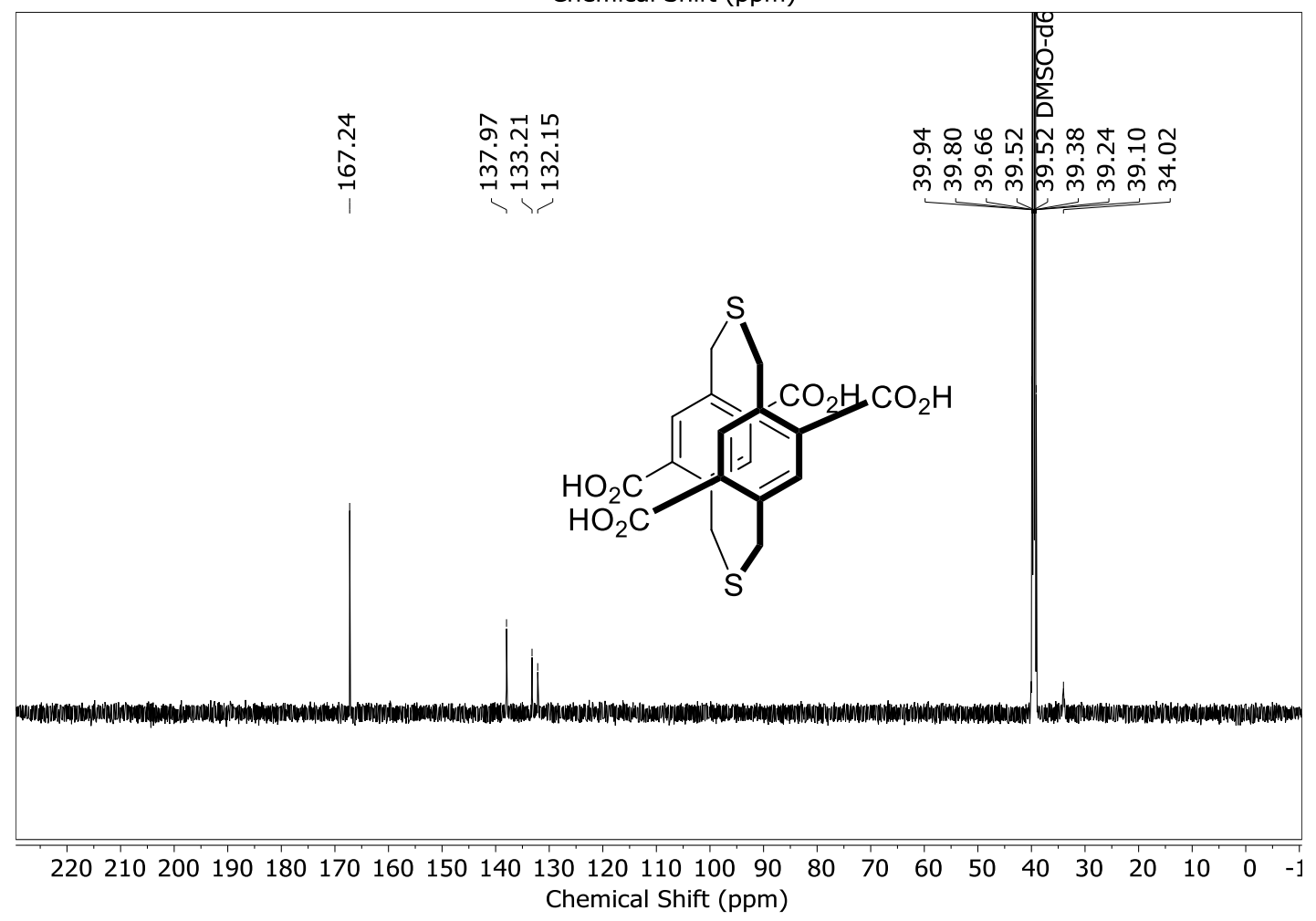




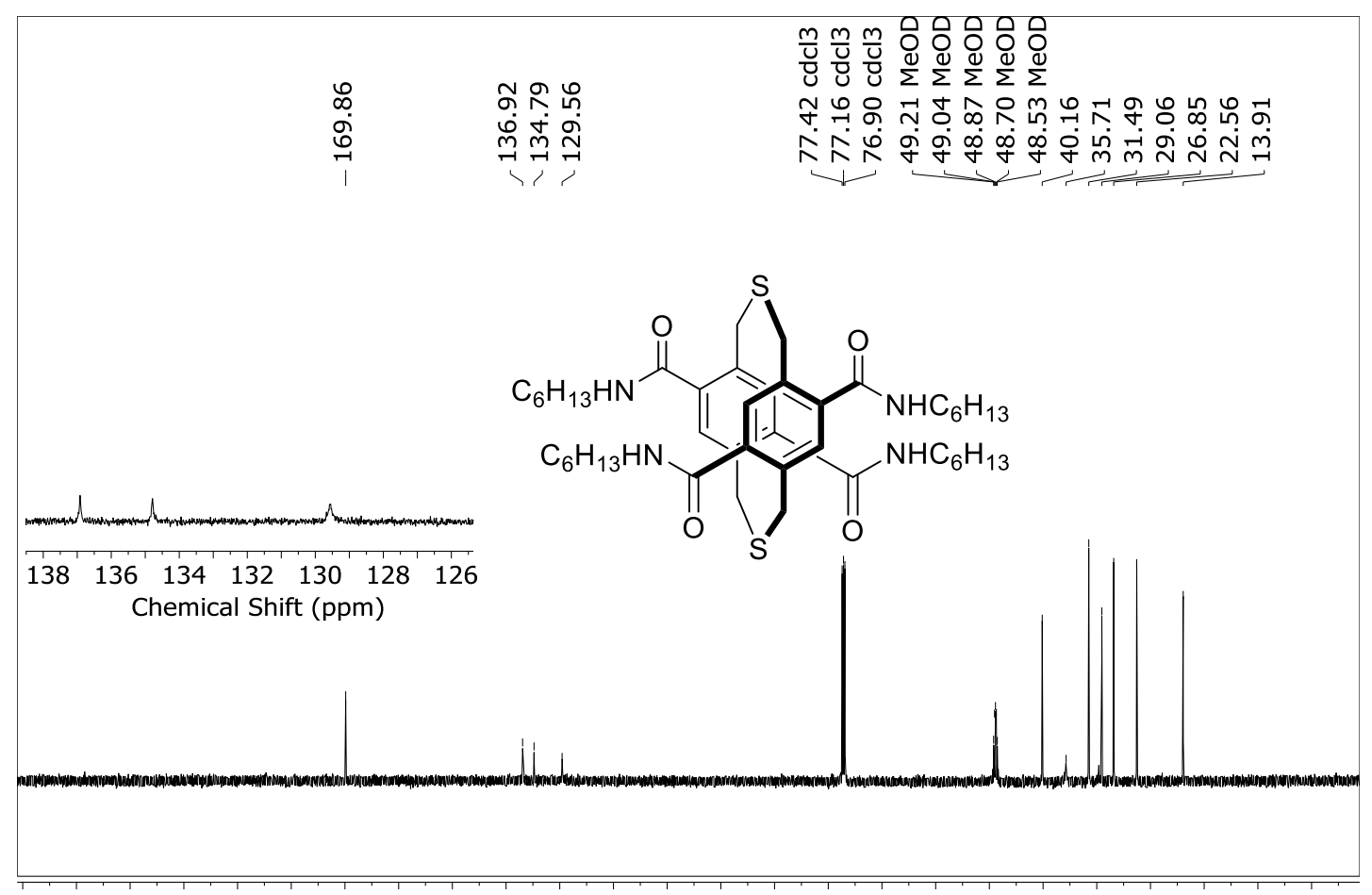

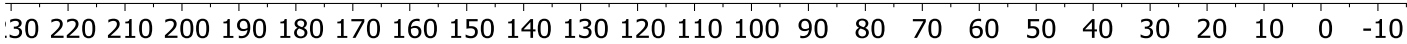
Chemical Shift (ppm)

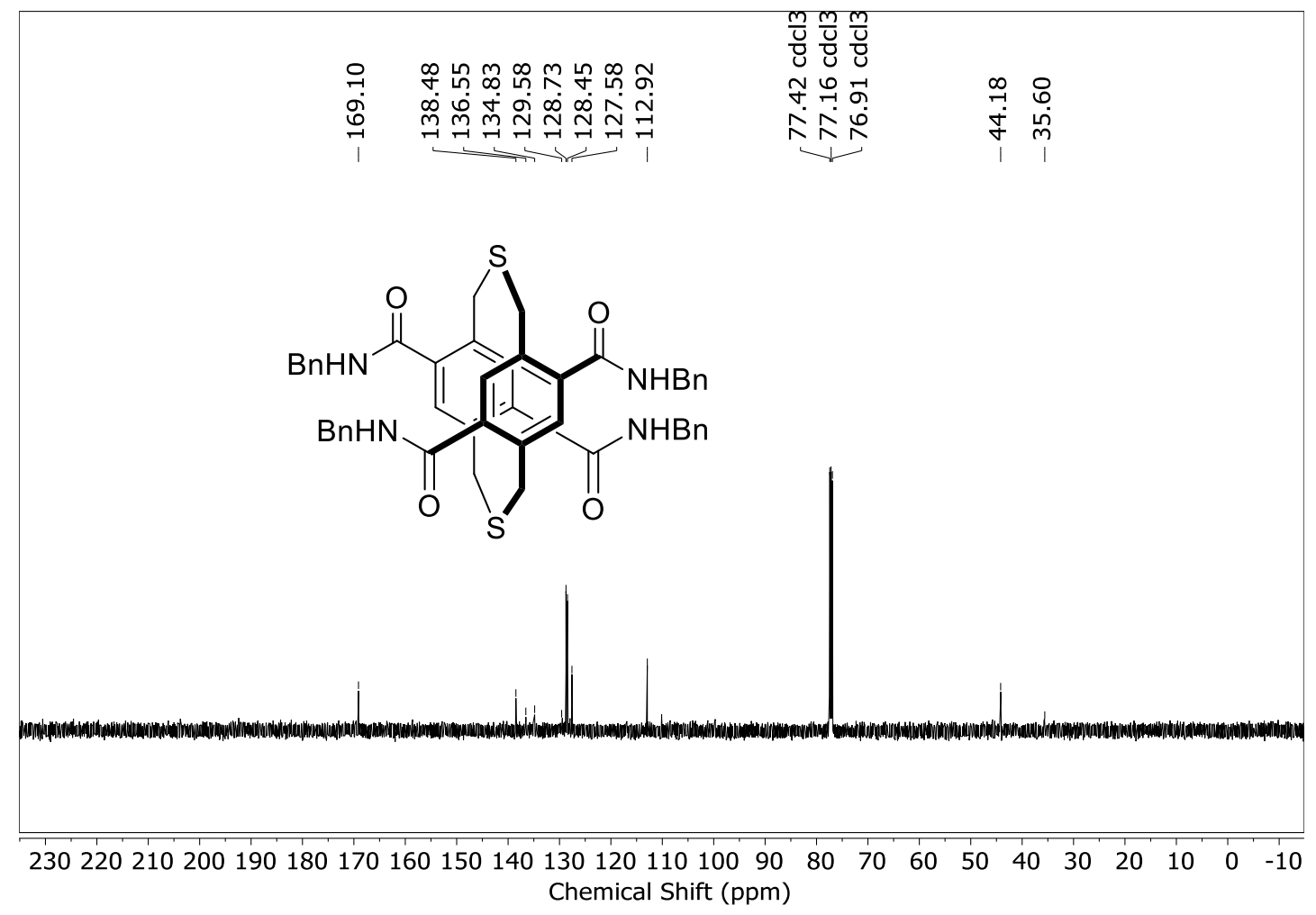




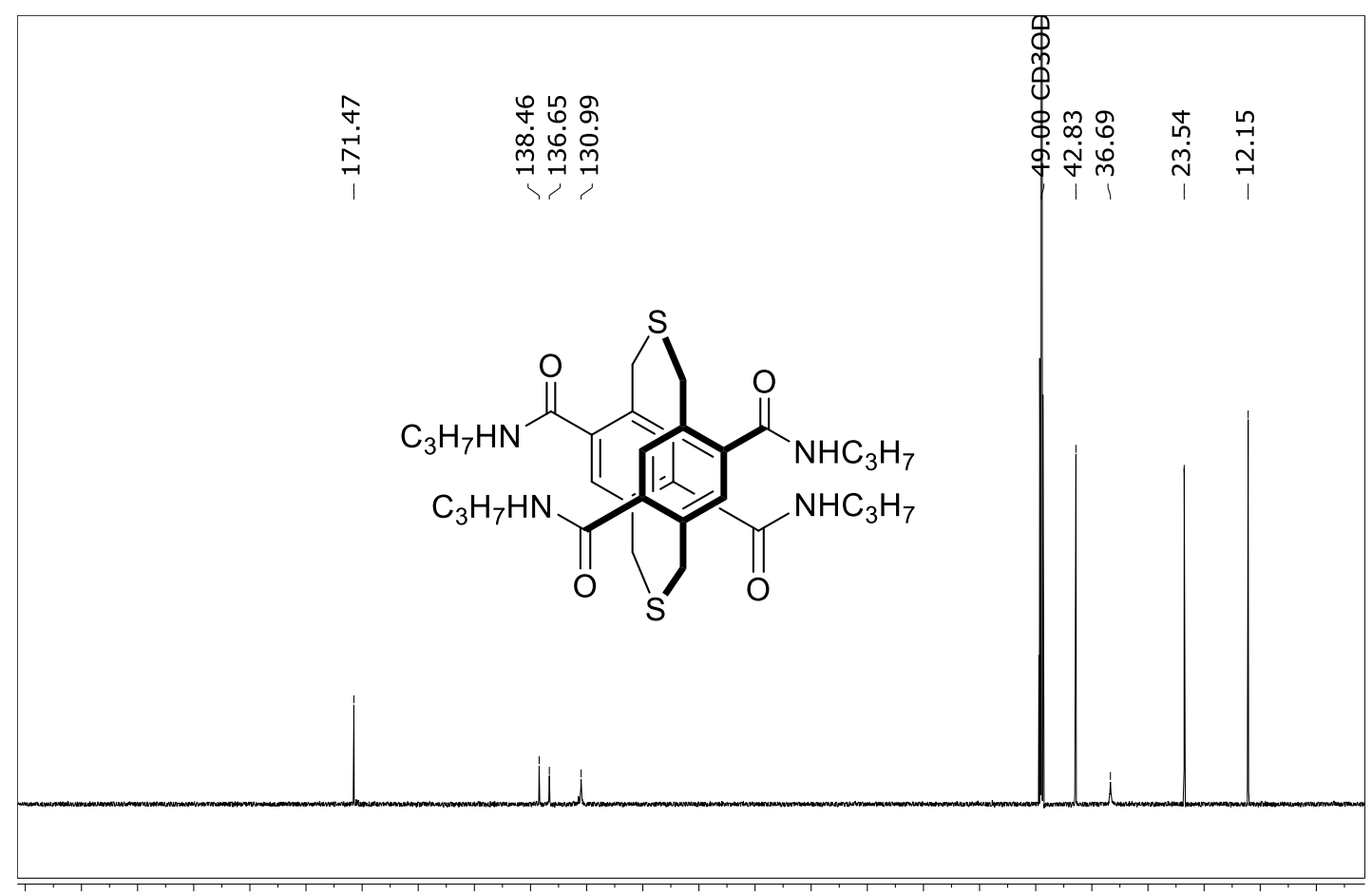

!30 220 210 200 $190180170160150140130120110100 \quad 90 \quad 80 \quad 70 \quad 60 \begin{array}{llllllll}10 & 40 & 30 & 20 & 10 & 0\end{array}$ Chemical Shift (ppm)

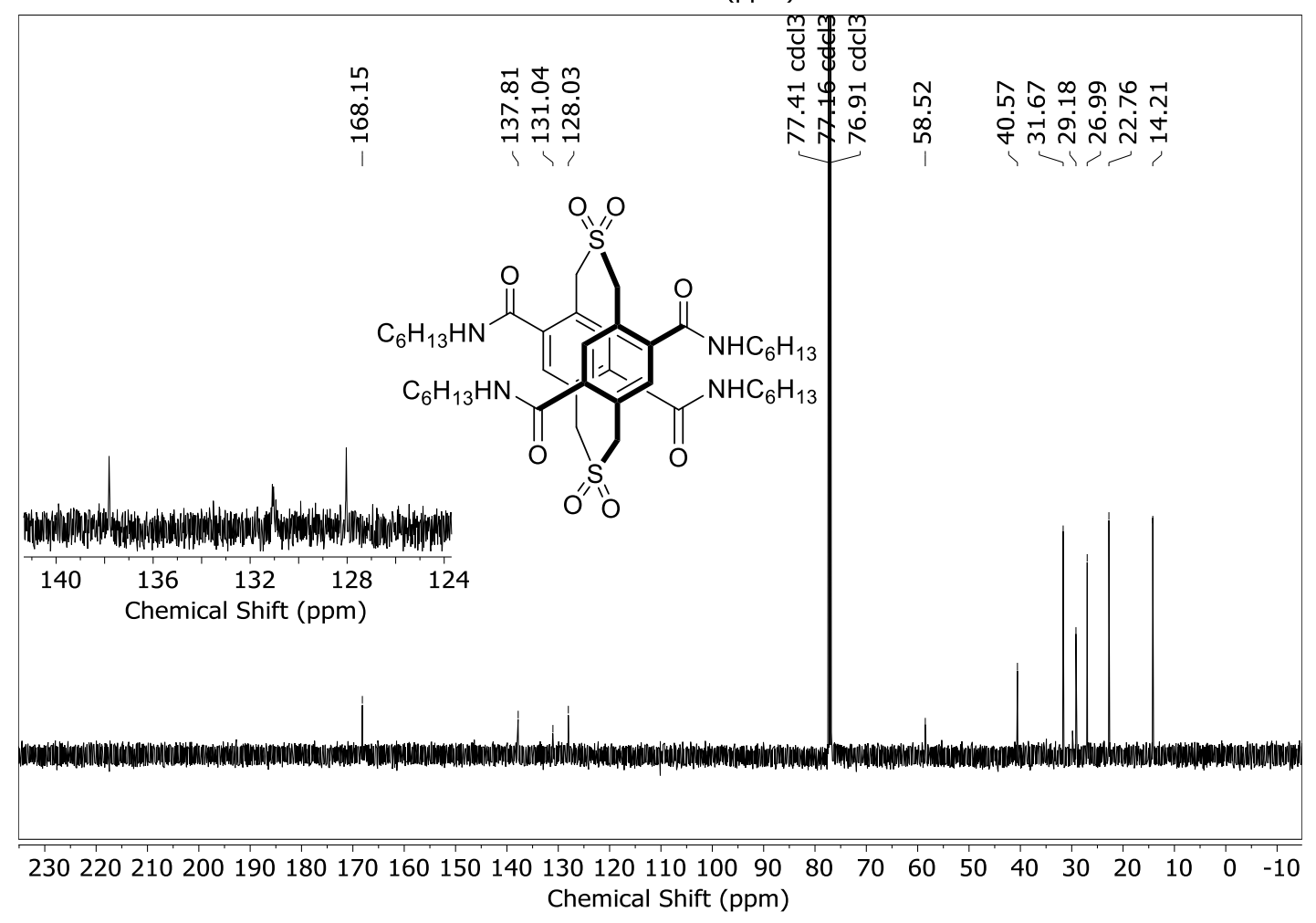




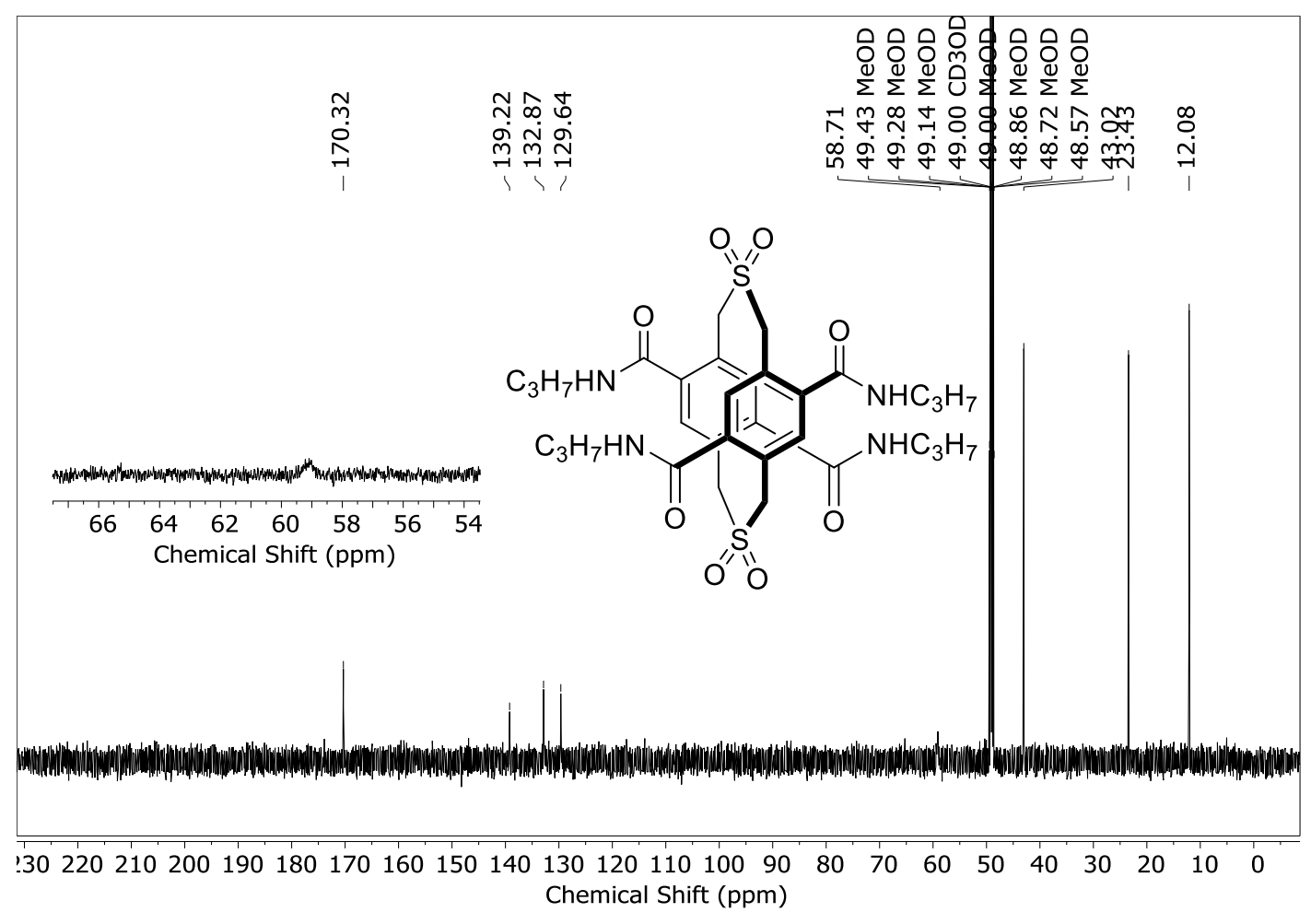




\section{Characterization of Bis(thio)lactone}<smiles>O=C1SCc2cc3c(cc21)CSC3=O</smiles>

\section{3,7-dihydro-1H,5H-benzo[1,2-c:4,5-c']dithiophene-1,5-dione}

In a $100 \mathrm{~mL}$ round bottom flask was placed 2,5-bis(thiomethyl)terephthalic acid $(0.83 \mathrm{~g}, 3.2 \mathrm{mmol})$. The residue was dissolved in $8 \mathrm{~mL}$ conc. $\mathrm{H}_{2} \mathrm{SO}_{4}$ and stirred for 1 hour before $50 \mathrm{~mL}$ of $\mathrm{MeOH}$ was slowly added. Light gray/tan crystals precipitated from solution and were collected by vacuum filtration to yield $0.71 \mathrm{~g}$ (quant.) of product. The incredible insolubility of the crystalline powder prevented full characterization. ${ }^{1} \mathrm{H}$ NMR (600 MHz, $\left.\mathrm{CDCl}_{3}\right) \delta 7.96$ (s, 2H), 4.57 (s, 4H). IR (ATR): $3032(\mathrm{w}), 2926$ (w), $1717(\mathrm{~m}), 1664(\mathrm{~s}), 1428(\mathrm{~m}), 1402(\mathrm{~m})$. GC/EI MS $\mathrm{m} / z$ calc'd 222.0 found 222.0.

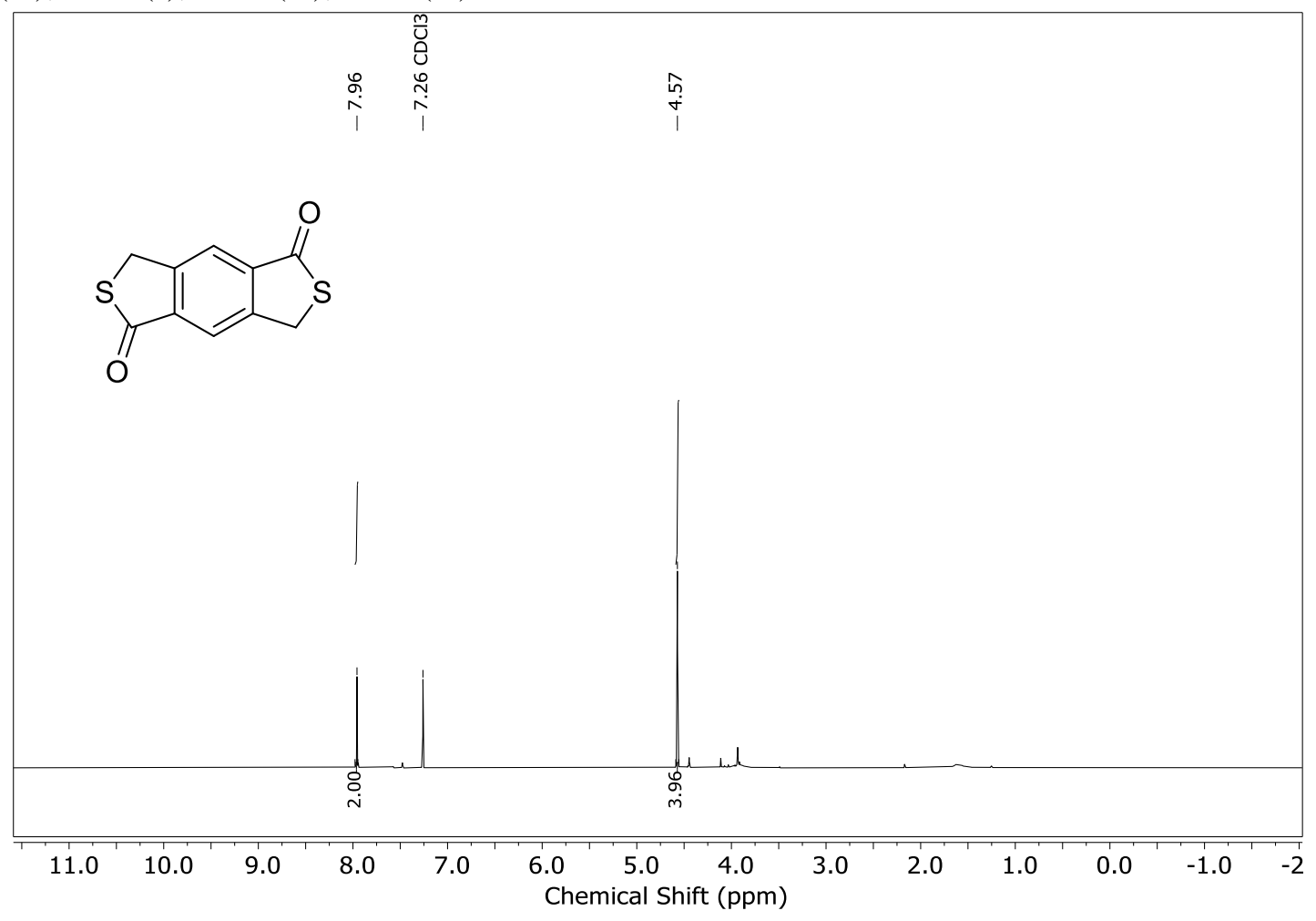




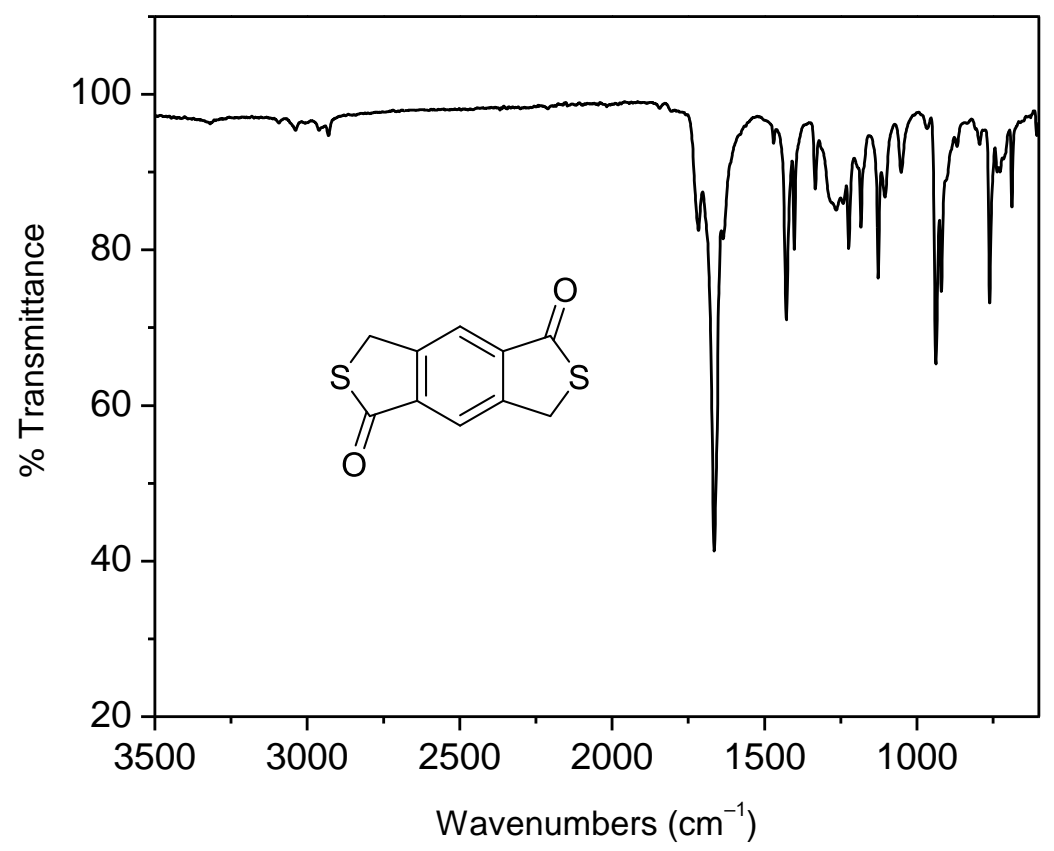

28999-GCMS-2019-0129-04

Rxi-5MS;50C $(0-3 m)>330 C @ 20 C / m$; hold 5; El-MS

28999-GCMS-2019-0129-04 \#1413-1427 RT: 1

1/29/2019 5:26:00 PM

WH2-135; 0.2 uL injected

+ c Full ms [10.00-700.00]

17.28-17.54 NL: 3.10E6

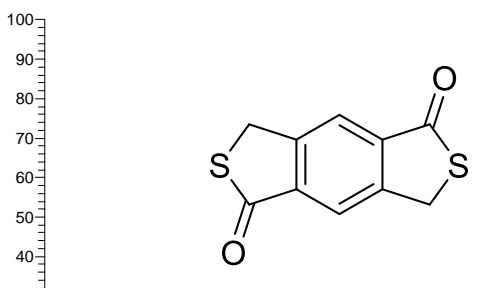

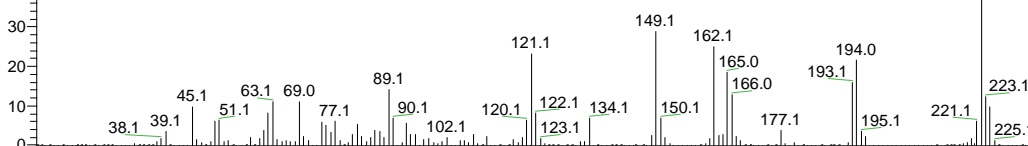
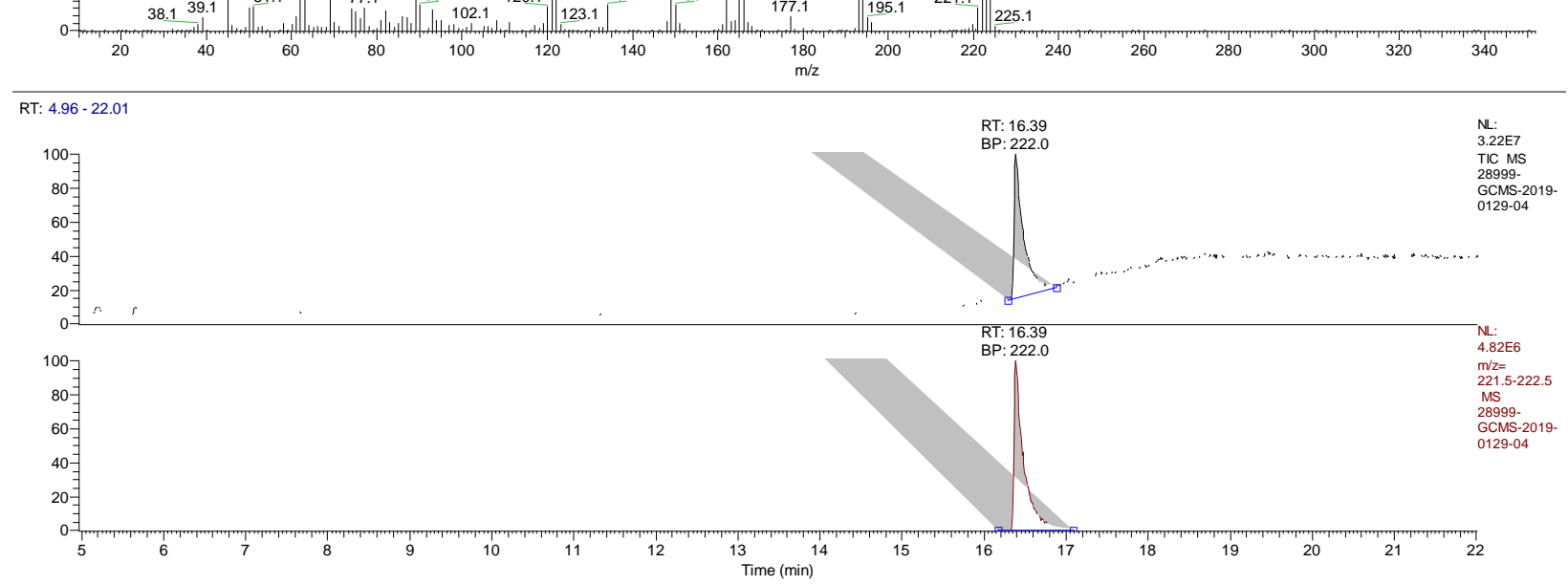
NMR Studies

\section{Assignment of Regiochemistry in $\mathbf{3}$ and $\mathbf{4}$}

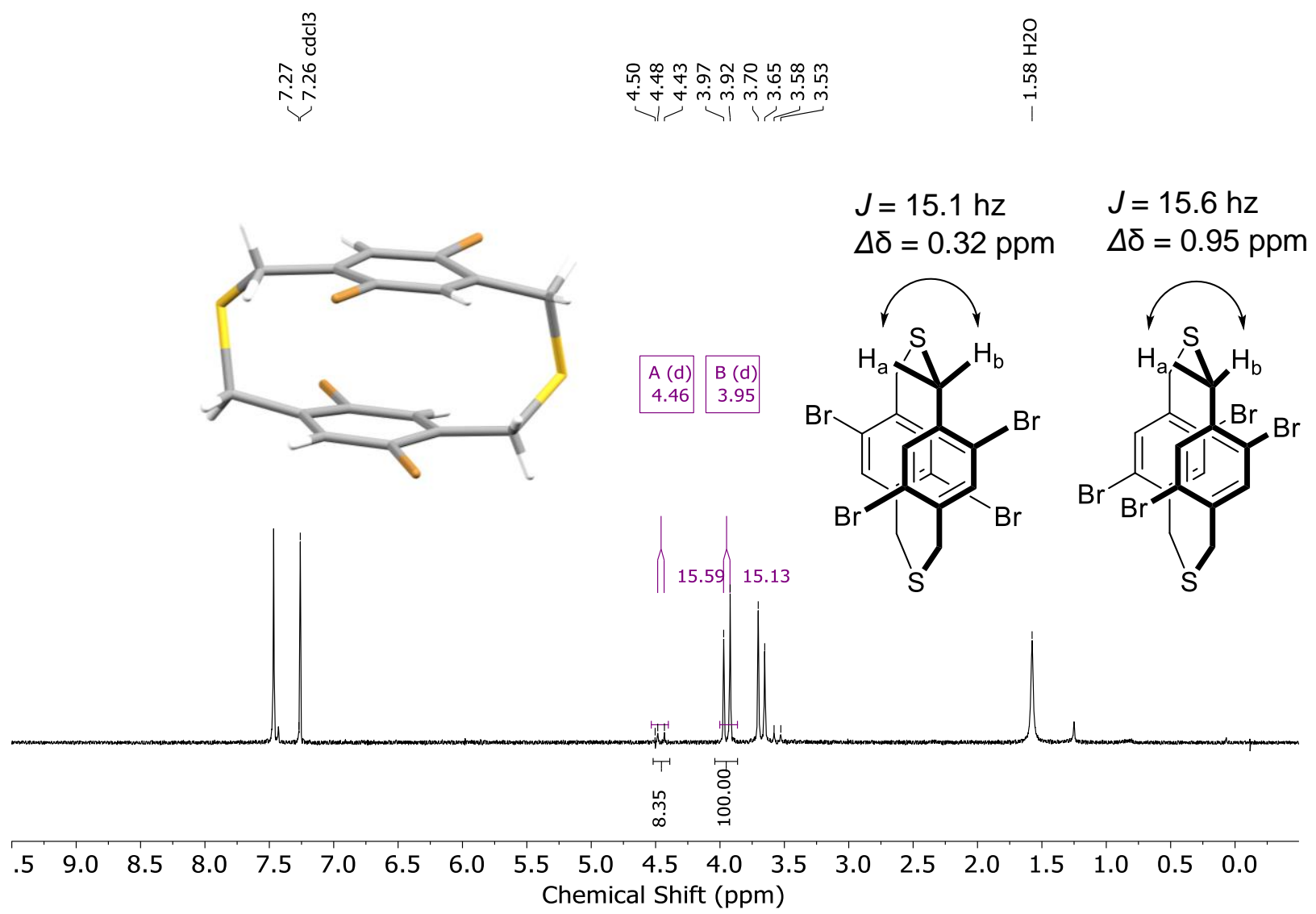

Figure S1. ${ }^{1} \mathrm{H}$ NMR (300 MHz) spectra of a mixture of ( \pm )-5,8,14,17-tetrabromo-dt[3.3]pCp and 5,8,15,18-tetrabromodt[3.3]pCp $c a$. 12:1 synthesized according to literature procedures. ${ }^{4}$ The difference in chemical shift between $\mathrm{H}_{\mathrm{a}}$ and $\mathrm{Hb}_{\mathrm{b}}$ as well as their gem- coupling constant is informative on the regiochemistry of the products. Structure of $( \pm)-5,8,14,17$-tetrabromodt[3.3]pCp was confirmed by X-ray crystallography (inset, CCDC 189356). 


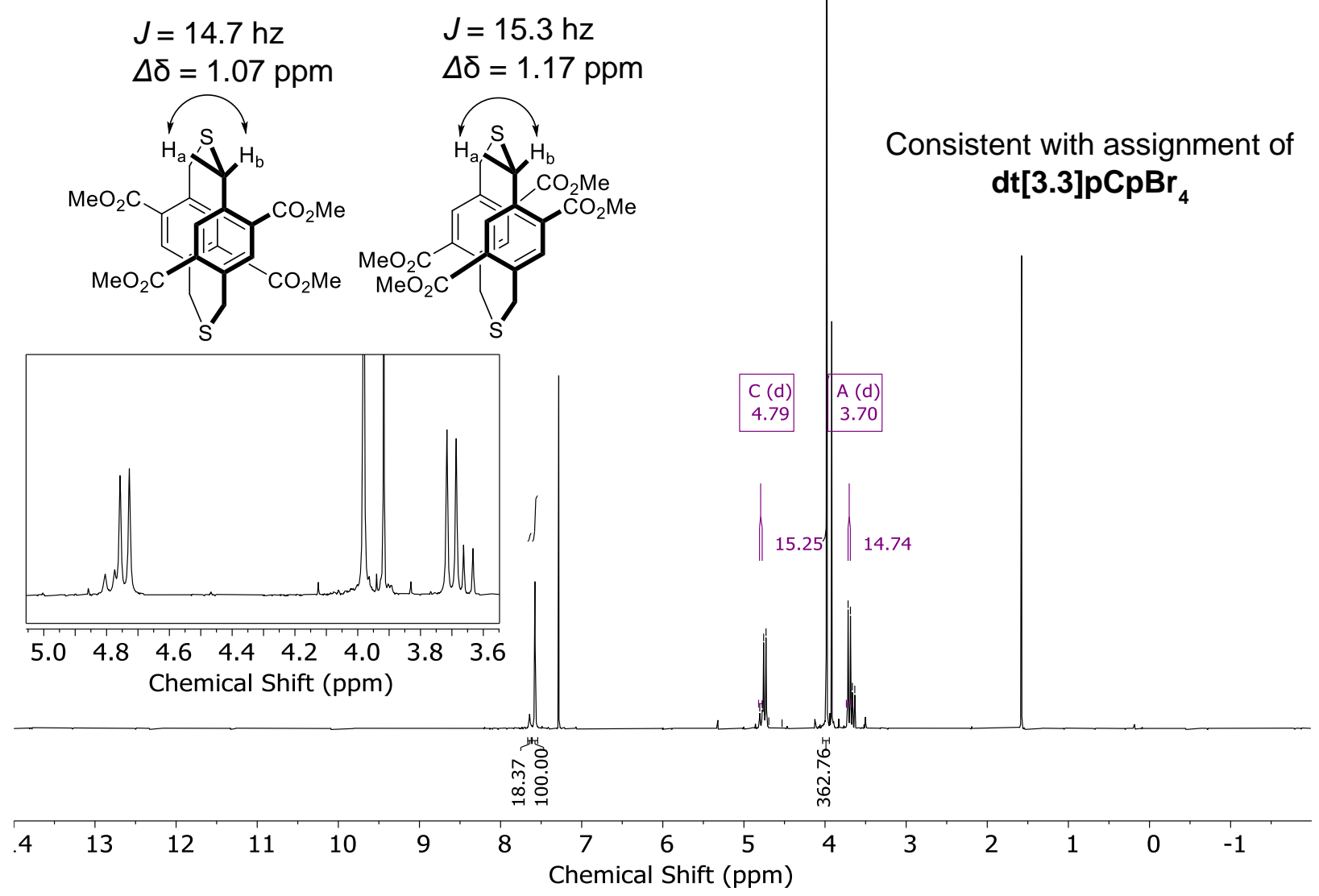

Figure S2. ${ }^{1} \mathrm{H}$ NMR (500 MHz) spectra of a mixture of ( \pm )-4a and $\mathbf{4 b}$ in a ca. 5:1 ratio with the coupling constants of $\mathrm{H}_{\mathrm{a}}$ and $\mathrm{Hb}_{\mathrm{b}}$ and difference in chemical shift between the two structures. The larger coupling constant and larger difference in chemical shift in the gem-isomer $\mathbf{4 b}$ are consistent with the regiochemical assignment of the structures from Figure S1. 


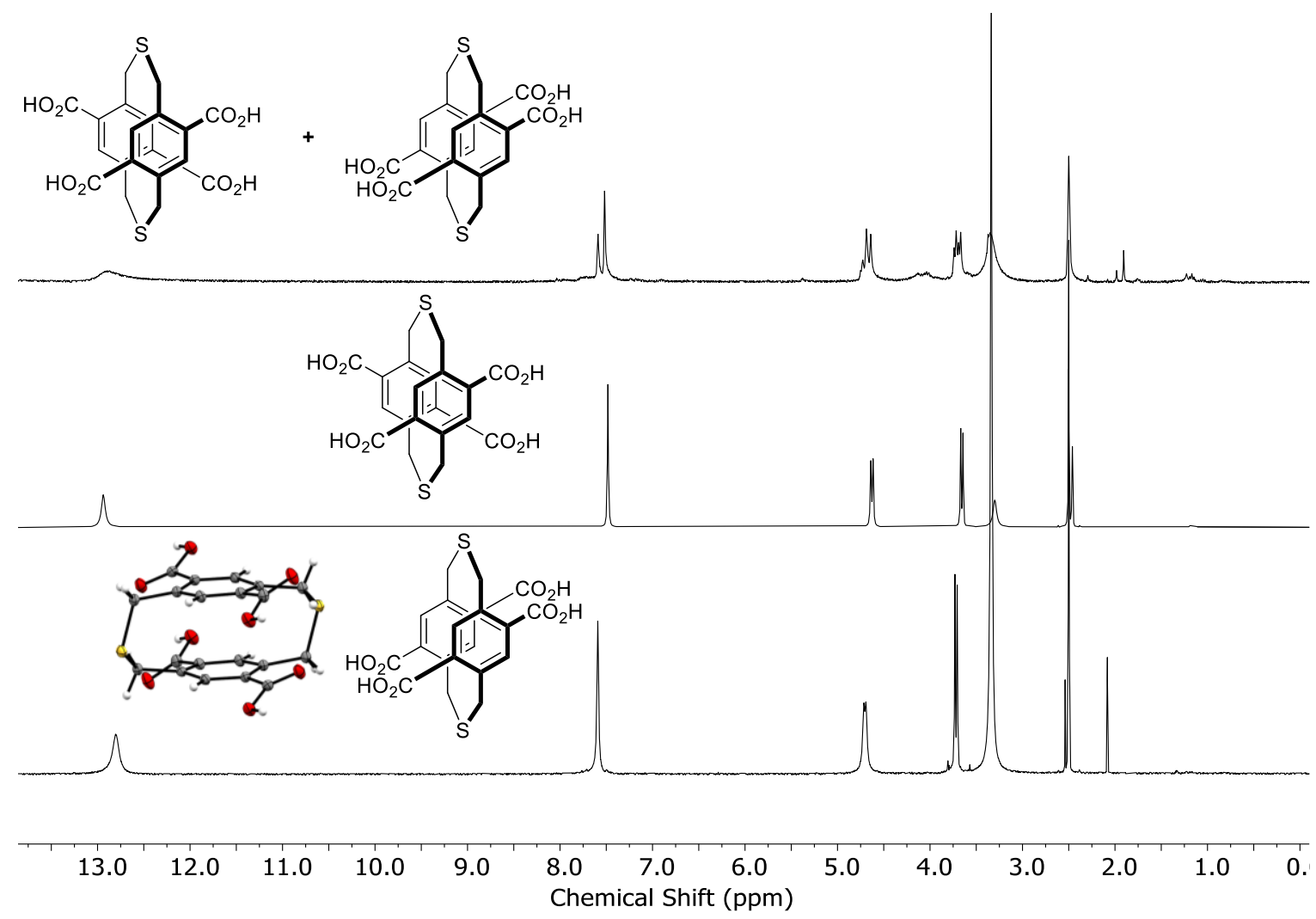

Figure S3. Comparison of the ${ }^{1} \mathrm{H}$ NMR of a mixture of ( \pm )-3a and $\mathbf{3 b}$ (top) ( $\mathbf{(}$ )-3a (middle) and $\mathbf{3 b}$ (bottom) with an ORTEP structure of $\mathbf{3 b}$ confirmed by X-ray crystallography. 


\section{Variable Concentration ${ }^{1} \mathrm{H}$ NMR Studies}

Variable concentration (at $298 \mathrm{~K}$ ) and variable temperature NMR studies were performed on an Inova $500 \mathrm{MHz}$ spectrometer with a Varian $5 \mathrm{~mm}$ conventional probe $\mathrm{H} 1 / \mathrm{P} 31-\mathrm{N} 15 . \mathrm{CDCl}_{3}$ and cyclohexane- $d 12$ were purchased from Sigma-Aldrich. Association constants were calculated using non-linear curve fitting in Origin 8.5 in accord with a dimerization and isodesmic polymerization association model (see below for details). ${ }^{5}$

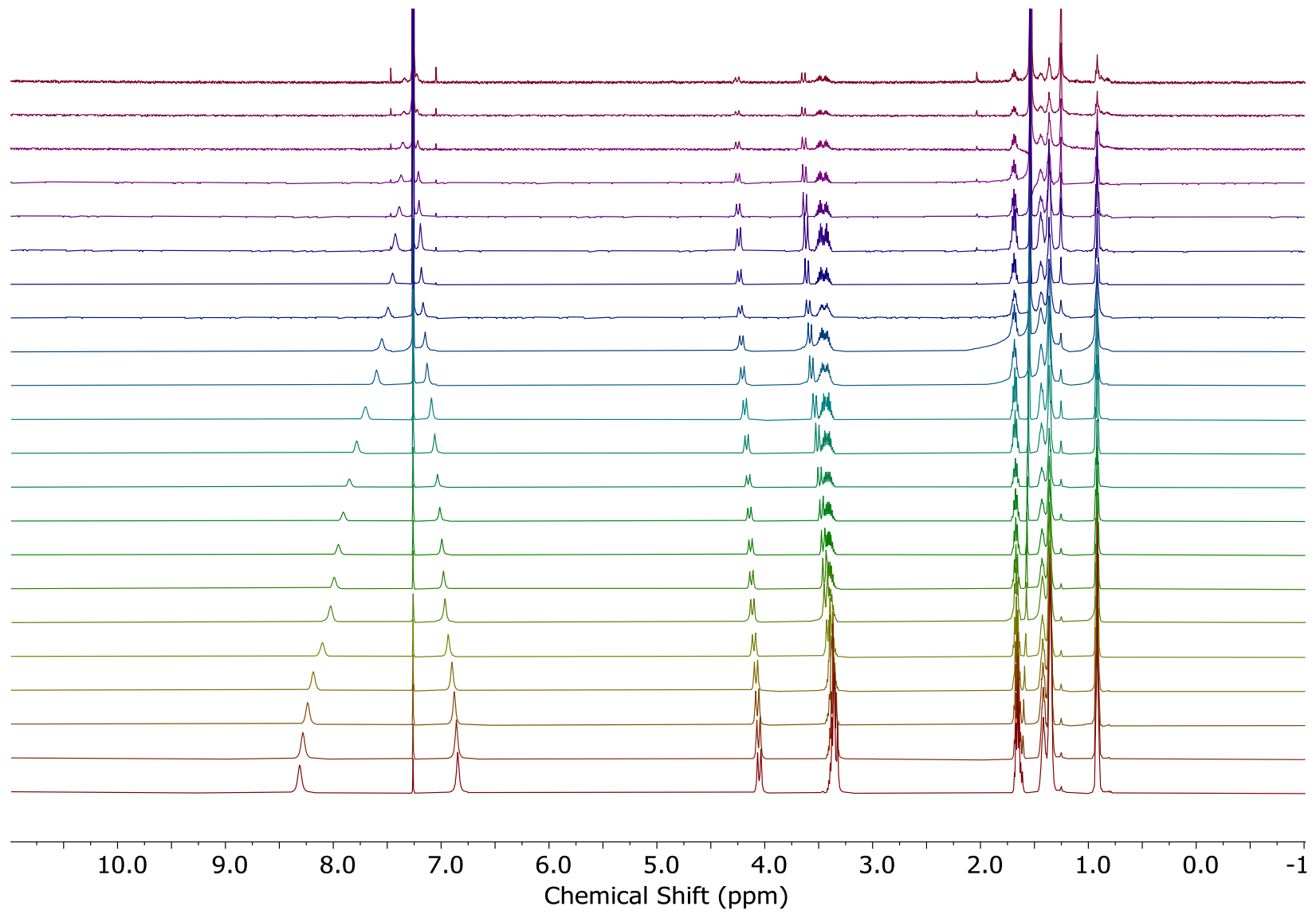

Figure S4. Variable concentration ${ }^{1} \mathrm{H}$ NMR of ( \pm )-1a in $\mathrm{CDCl}_{3}$ from $0.025-24 \mathrm{mM}$ (top to bottom) measured at $298 \mathrm{~K}$. 


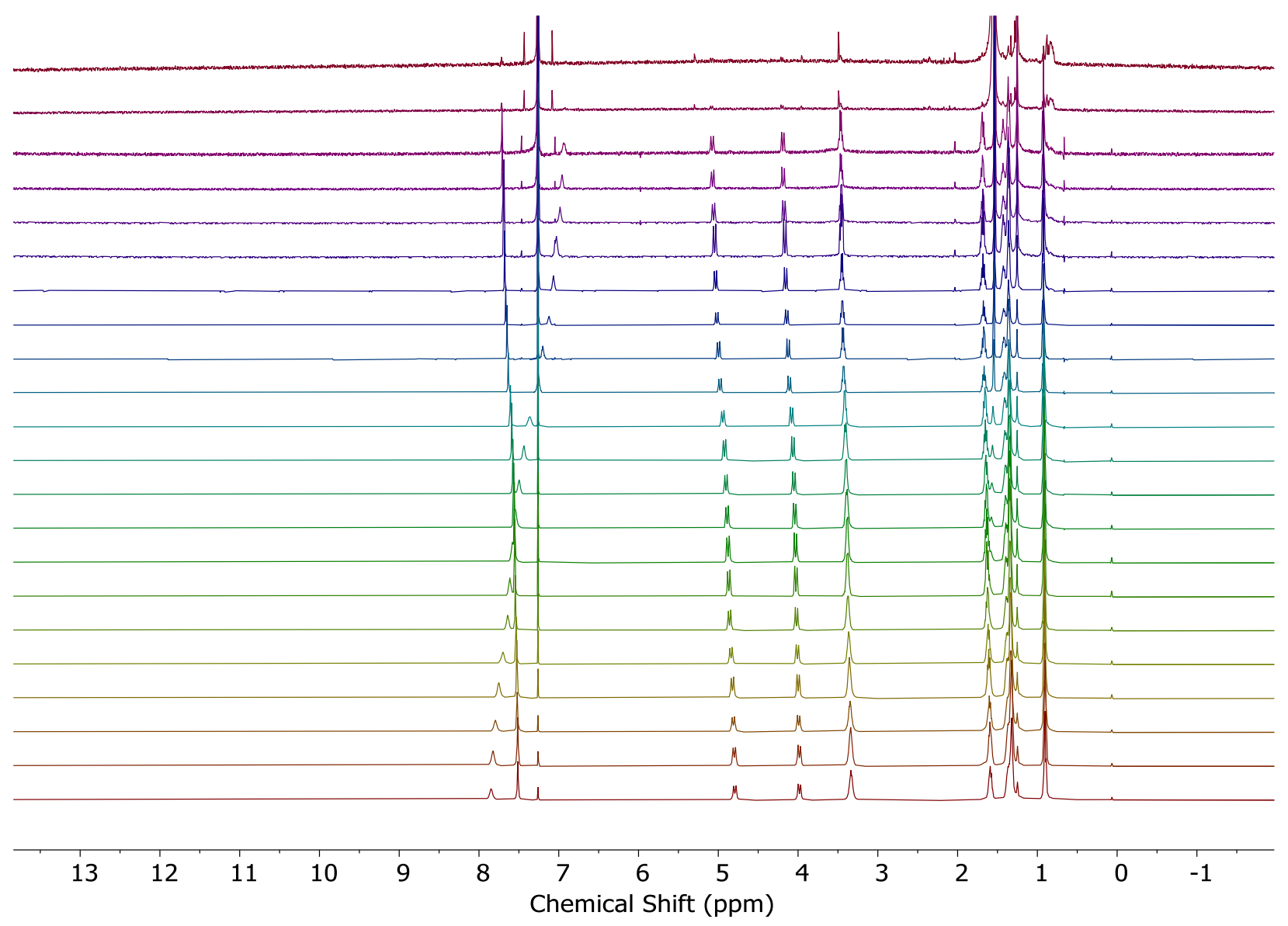

Figure S5. Variable concentration ${ }^{1} \mathrm{H}$ NMR of ( \pm )-2a from 0.025-24 mM (top to bottom) measured at $298 \mathrm{~K}$. 


\section{Variable Temperature ${ }^{1} \mathrm{H}$ NMR Studies}

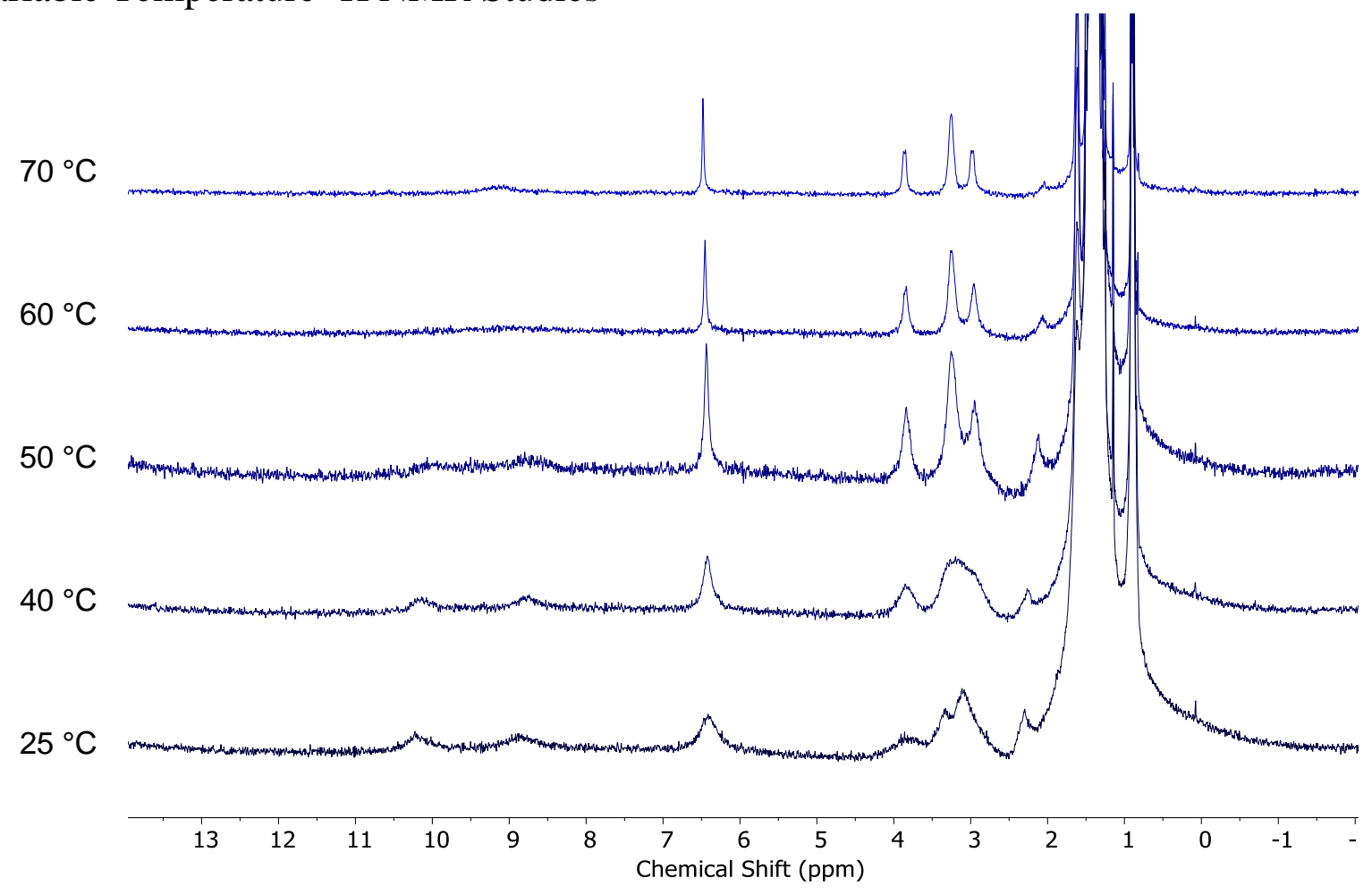

Figure S6. Variable temperature ${ }^{1} \mathrm{H}$ NMR of $( \pm)-1 a 1 \mathrm{mM}$ in cyclohexane- $d_{12}$ from $25-70{ }^{\circ} \mathrm{C}$.

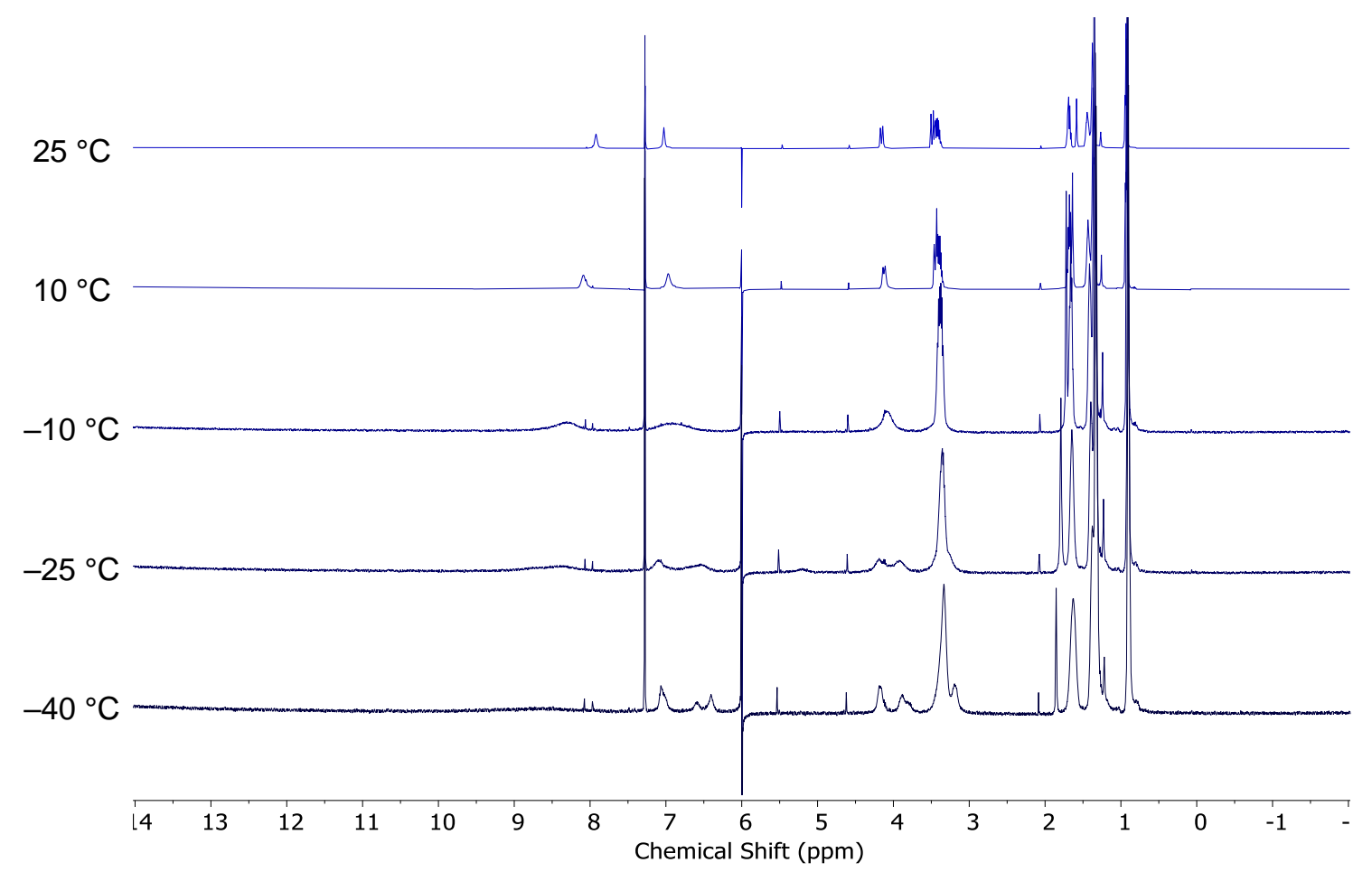

Figure S7. Variable temperature ${ }^{1} \mathrm{H}$ NMR of ( \pm )-1a $10 \mathrm{mM}$ in $\mathrm{CDCl}_{3}$ from $-40-25{ }^{\circ} \mathrm{C}$. 

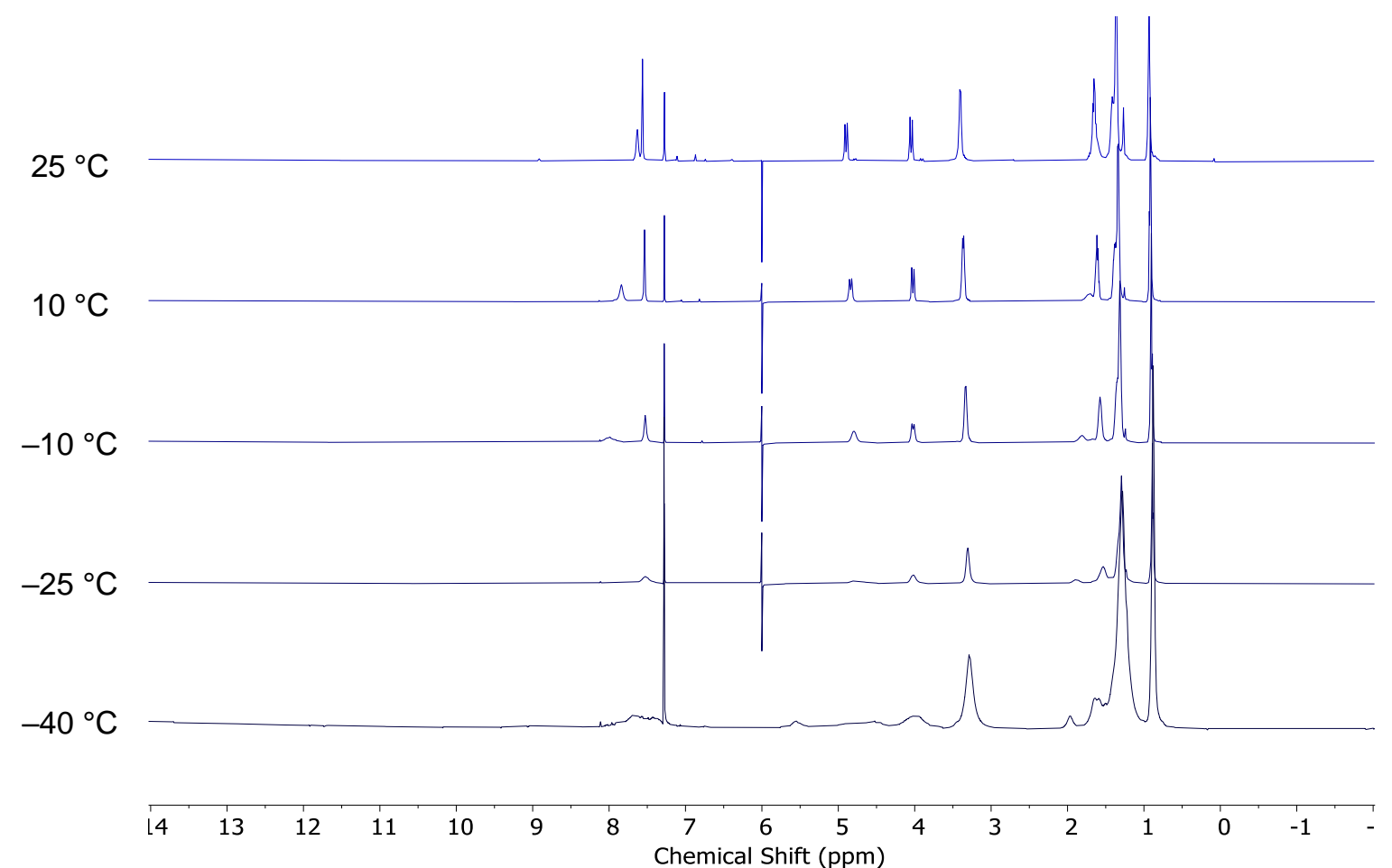

Figure S8. Variable temperature ${ }^{1} \mathrm{H}$ NMR of (土)-2a $10 \mathrm{mM}$ in $\mathrm{CDCl}_{3}$ from $-40-25{ }^{\circ} \mathrm{C}$. 


\section{Determination of Association Constants}

The concentration-dependent chemical shift data collected for ( \pm )-1a was fit to a dimerization model to determine $K_{\mathrm{d}}(e q .1)^{6}$ and an isodesmic polymerization model to determine $K_{\mathrm{e}}(e q .2){ }^{7} \delta_{\text {obs }}=$ observed chemical shift,$\delta_{\mathrm{m}}=$ chemical shift of monomer (chosen as the lowest concentration), $\delta_{\mathrm{d}}=$ chemical shift of dimer, $\delta_{\mathrm{agg}}=$ chemical shift of aggregate, $c_{\mathrm{t}}=$ total concentration, $K_{\mathrm{d}}=$ dimerization equilibrium constant, $K_{\mathrm{e}}=$ elongation equilibrium constant.

eq. 1

$$
\delta_{o b s}=\delta_{d}+\left(\delta_{m}-\delta_{d}\right)\left[\frac{-1+\sqrt{1+8 K_{d} C_{t}}}{4 K_{d} C_{t}}\right]
$$

eq. 2

$$
\delta_{o b s}=\delta_{m}+\left(\delta_{a g g}-\delta_{m}\right)\left[1+\frac{1-\sqrt{4 K_{e} C_{t}+1}}{2 K_{e} C_{t}}\right]
$$


(a)

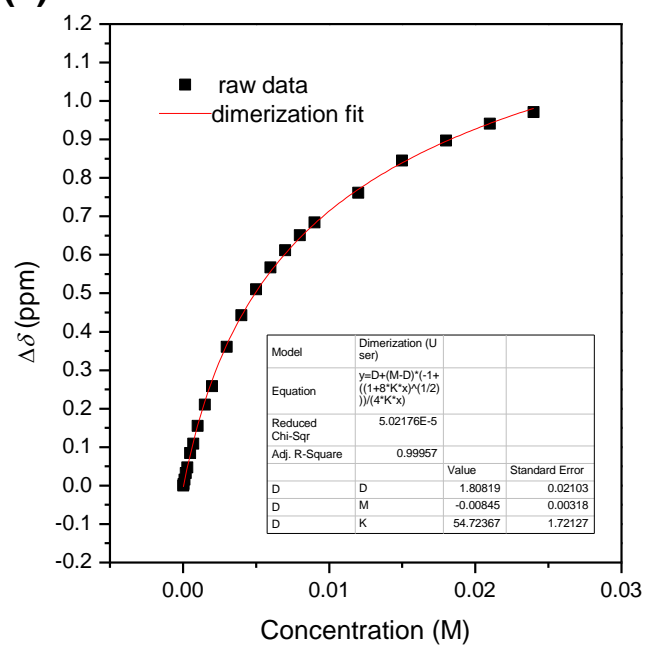

(c)

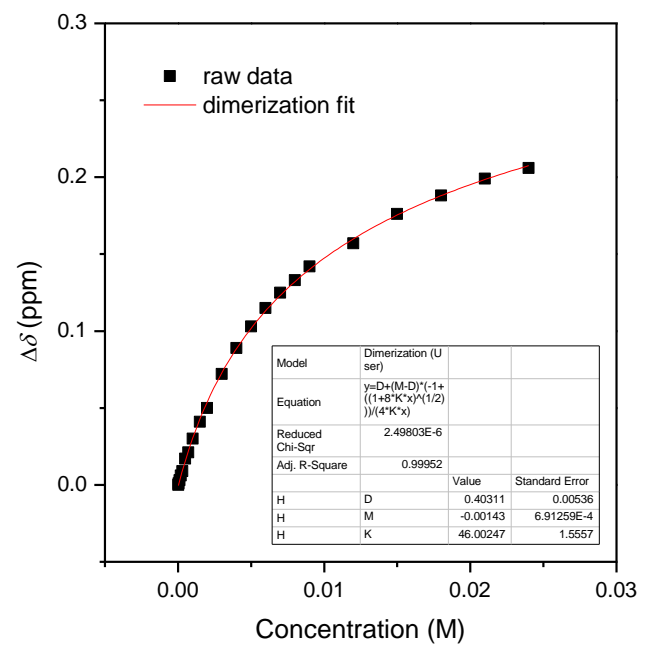

(b)

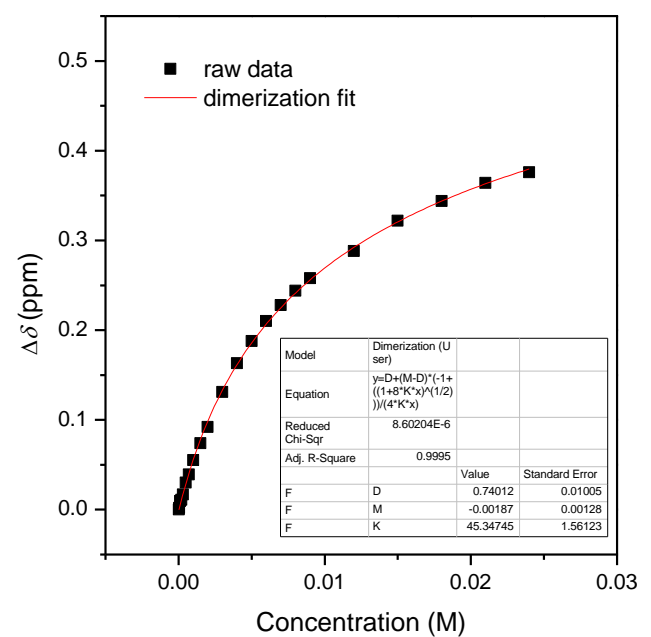

Figure S9. Fitting of key chemical shifts from the concentration dependent ${ }^{1} \mathrm{H}$ NMR of $( \pm)$-1a in Figure $\mathrm{S} 4$ to a dimerization model. Key chemical shifts are: $\mathrm{N}-\mathrm{H}_{\mathrm{a} / \mathrm{b}}(\mathrm{a}), \mathrm{C}-\mathrm{H}_{\mathrm{c} / \mathrm{d}}(\mathrm{b})$, and bridge $\mathrm{C}-\mathrm{H}_{\mathrm{e}}(\mathrm{c})$. 
(a)

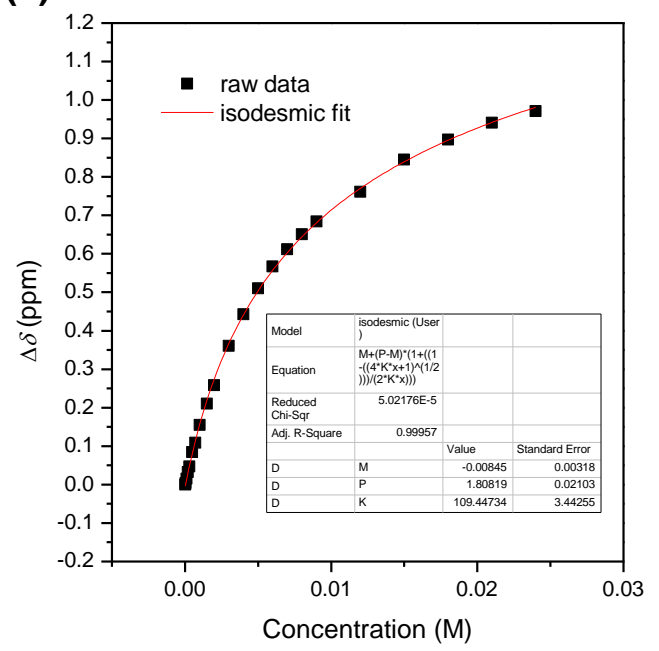

(b)

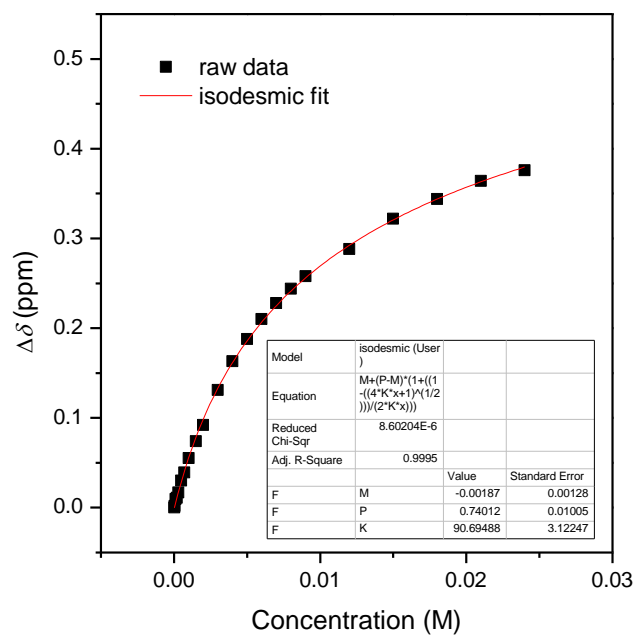

(c)

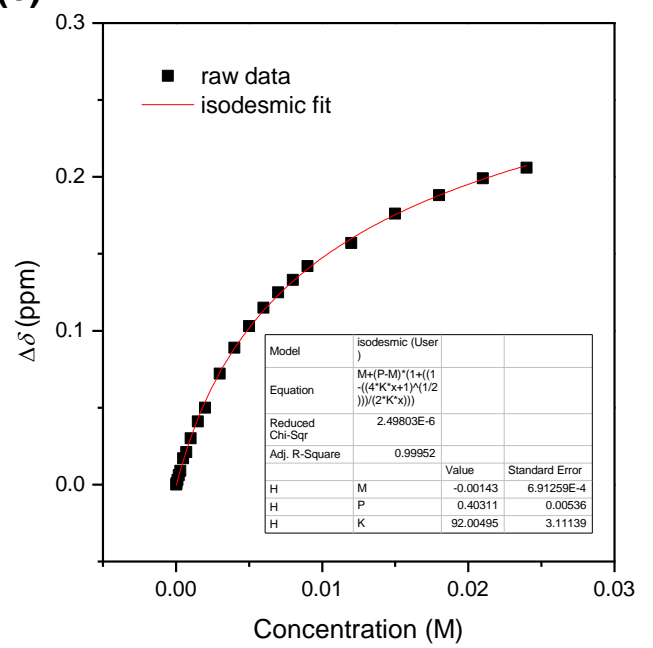

Figure S10. Fitting of the change of key chemical shifts from the concentration dependent ${ }^{1} \mathrm{H}$ NMR of ( \pm )-1a in Figure S4 to an isodesmic model. Key chemical shifts are: $\mathrm{N}-\mathrm{H}_{\mathrm{a} / \mathrm{b}}(\mathrm{a}), \mathrm{C}-\mathrm{H}_{\mathrm{c} / \mathrm{d}}(\mathrm{b})$, and bridge $\mathrm{C}-\mathrm{H}_{\mathrm{e}}$ (c).

Table S1. Summary of elongation constants $\left(K_{\mathrm{e}}\right)$ and dimerization constants $\left(K_{\mathrm{d}}\right)$ from fitting of variable concentration ${ }^{1} \mathrm{H}$ NMR experiment in Figure S1.

\begin{tabular}{|c|c|c|c|}
\hline Resonance & $\boldsymbol{K}_{\mathbf{e}}$ & $\boldsymbol{K}_{\text {dim }}$ & $\boldsymbol{R}^{\mathbf{2}}$ \\
\hline \hline $\mathrm{N}-\mathrm{H}_{\mathrm{a} / \mathrm{b}}$ & $109 \pm 3$ & $55 \pm 2$ & 0.999 \\
\hline $\mathrm{C}-\mathrm{H}_{\mathrm{c} / \mathrm{d}}$ & $91 \pm 3$ & $45 \pm 2$ & 0.999 \\
\hline Bridge $\mathrm{C}-\mathrm{H}_{\mathrm{e}}$ & $92 \pm 3$ & $46 \pm 2$ & 0.999 \\
\hline
\end{tabular}


(a)

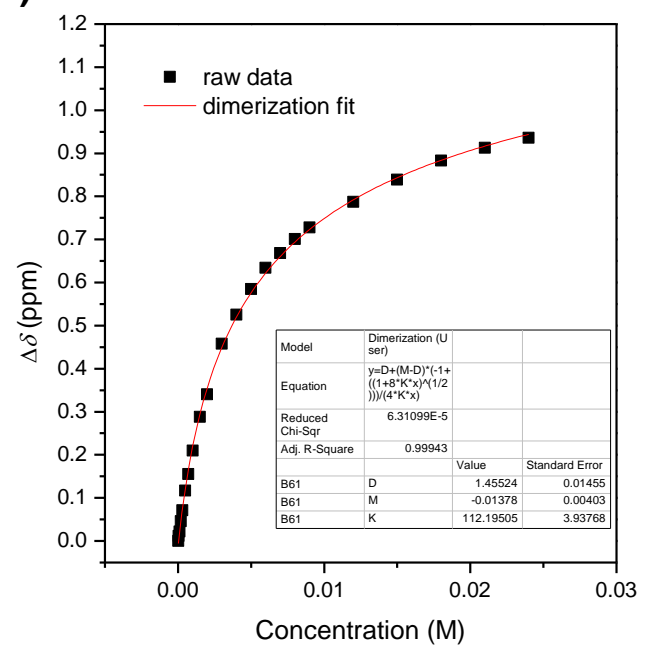

(c)

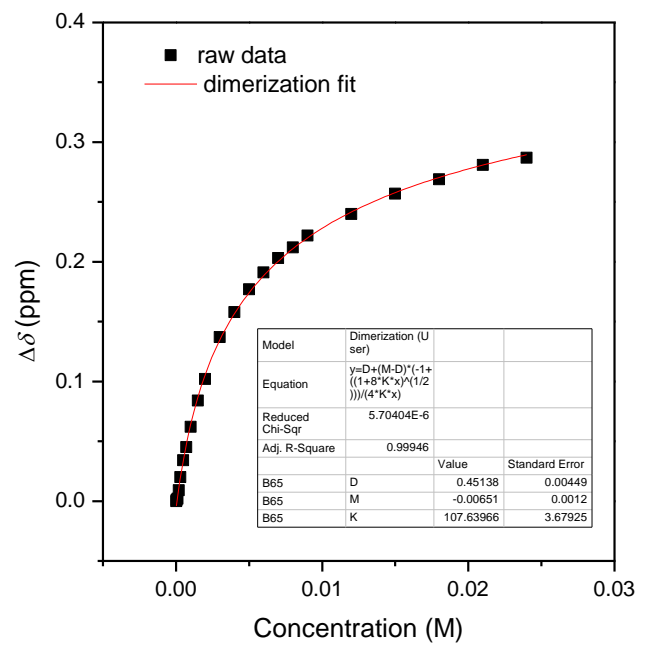

(b)

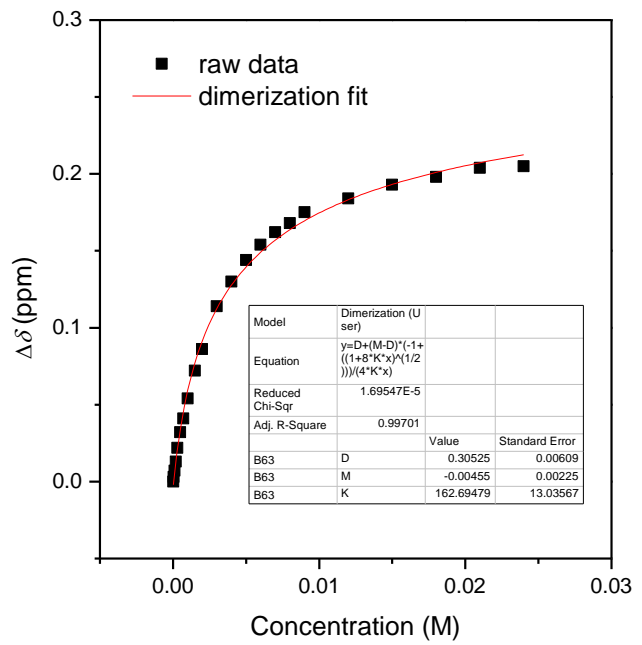

(d)

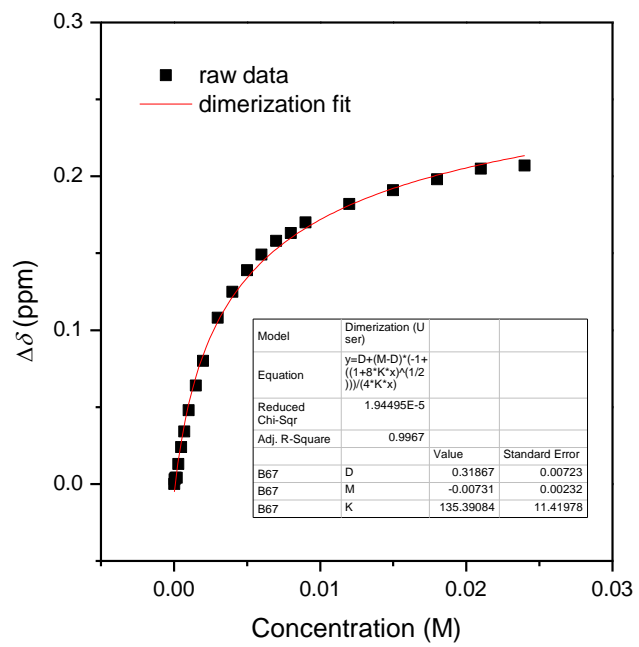

Figure S11. Fitting of the change in key chemical shift of ( \pm )-2a from Figure S5 to a dimerization model. Key chemical shifts are: $\mathrm{N}-\mathrm{H}_{\mathrm{a}^{\prime} \mathrm{b}^{\prime}}(\mathrm{a}), \mathrm{C}-\mathrm{H}_{\mathrm{c}^{\prime} / \mathrm{d}^{\prime}}(\mathrm{b})$, bridge $\mathrm{C}-\mathrm{H}_{\mathrm{e}^{\prime}}(\mathrm{c})$, and bridge $\mathrm{C}-\mathrm{H}_{\mathrm{f}^{\prime}}(\mathrm{d})$. 
(a)

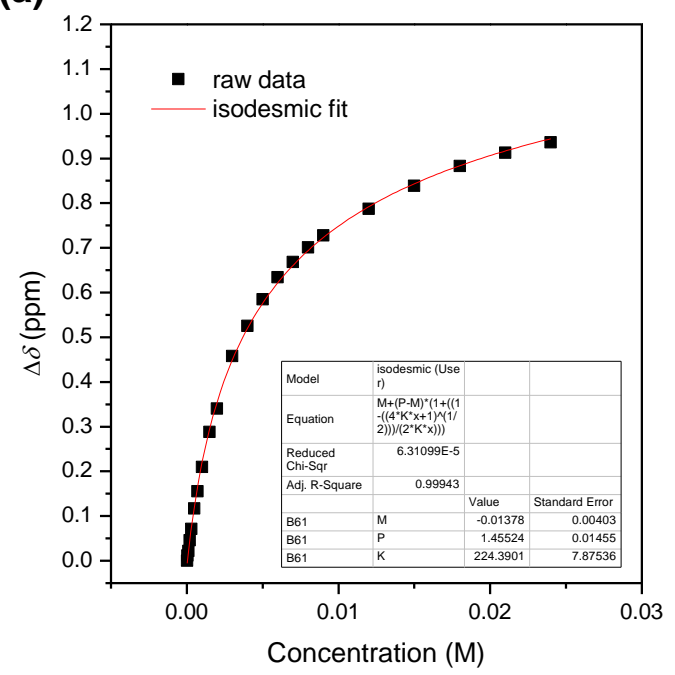

(c)

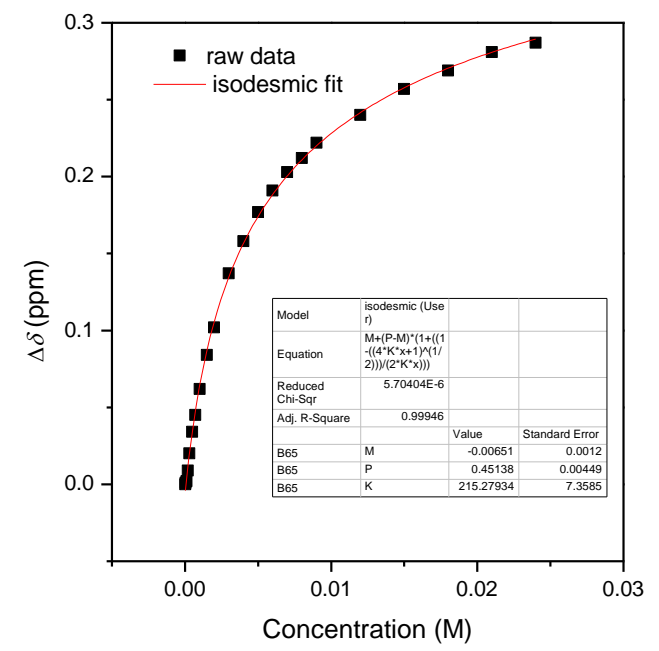

(b)

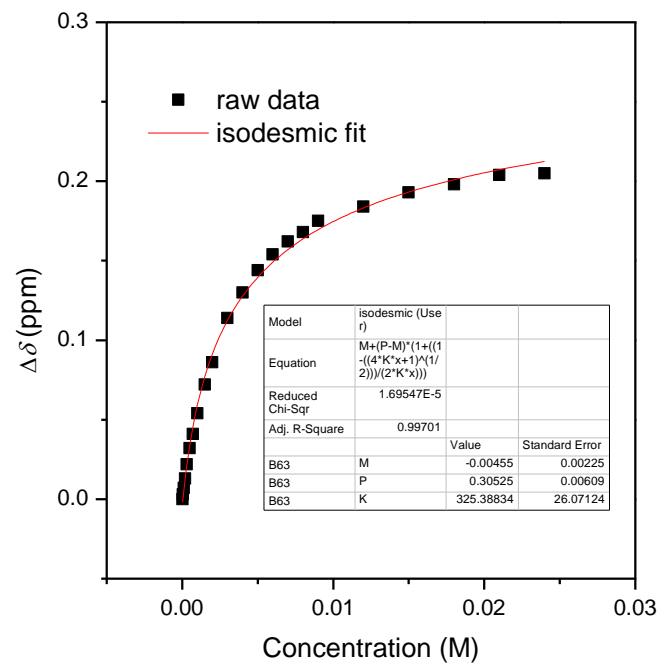

(d)

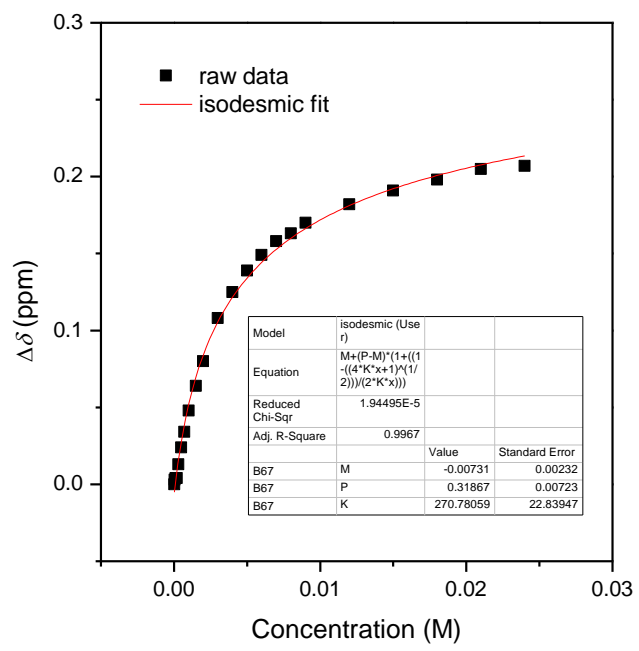

Figure S12. Fitting of the change in key chemical shift of ( \pm )-2a from Figure S5 to an isodesmic model. Key chemical shifts are: $\mathrm{N}-\mathrm{H}_{\mathrm{a}^{\prime} / \mathrm{b}^{\prime}}(\mathrm{a}), \mathrm{C}-\mathrm{H}_{\mathrm{c}^{\prime} / \mathrm{d}^{\prime}}(\mathrm{b})$, bridge $\mathrm{C}-\mathrm{H}_{\mathrm{e}^{\prime}}(\mathrm{c})$, and bridge $\mathrm{C}-\mathrm{H}_{\mathrm{f}^{\prime}}(\mathrm{d})$.

Table S2. Dimerization constants and elongation constants derived from fitting variable concentration ${ }^{1} \mathrm{H}$ NMR data of $( \pm)$-2a.in Figure S4 and S5.

\begin{tabular}{|c|c|c|c|}
\hline Resonance & $\boldsymbol{K}_{\mathbf{e}}$ & $\boldsymbol{K}_{\text {dim }}$ & $\mathbf{R}^{\mathbf{2}}$ \\
\hline \hline $\mathrm{N}-\mathrm{H}_{\mathrm{a}^{\prime} / \mathrm{b}^{\prime}}$ & $224 \pm 8$ & $112 \pm 4$ & 0.999 \\
\hline $\mathrm{C}-\mathrm{H}_{\mathrm{c}^{\prime} / \mathrm{d}^{\prime}}$ & $325 \pm 26$ & $163 \pm 13$ & 0.997 \\
\hline Bridge $\mathrm{C}-\mathrm{H}_{\mathrm{e}^{\prime}}$ & $215 \pm 7$ & $108 \pm 4$ & 0.999 \\
\hline Bridge $\mathrm{C}-\mathrm{H}_{\mathrm{f}^{\prime}}$ & $271 \pm 23$ & $135 \pm 11$ & 0.997 \\
\hline
\end{tabular}




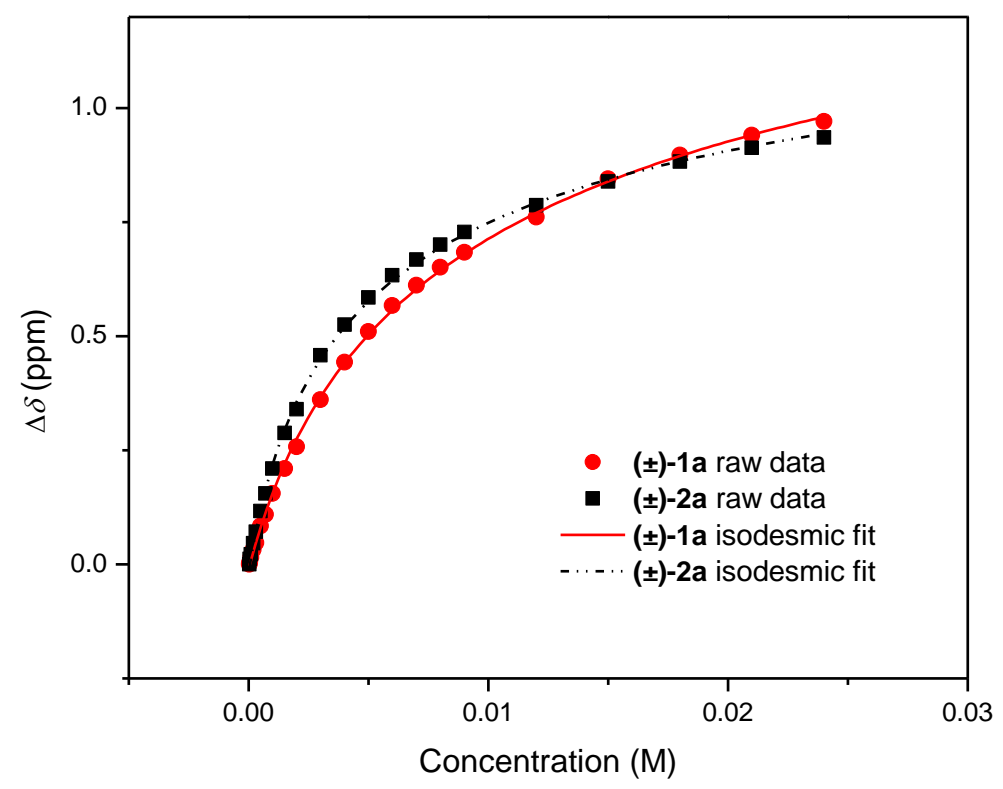

Figure S13. Overlaid plots of the change in chemical shift for the amide N-H in ( \pm )-1a and ( \pm )-2a from Figure S4 and Figure S5 and the corresponding fits to an isodesmic model.
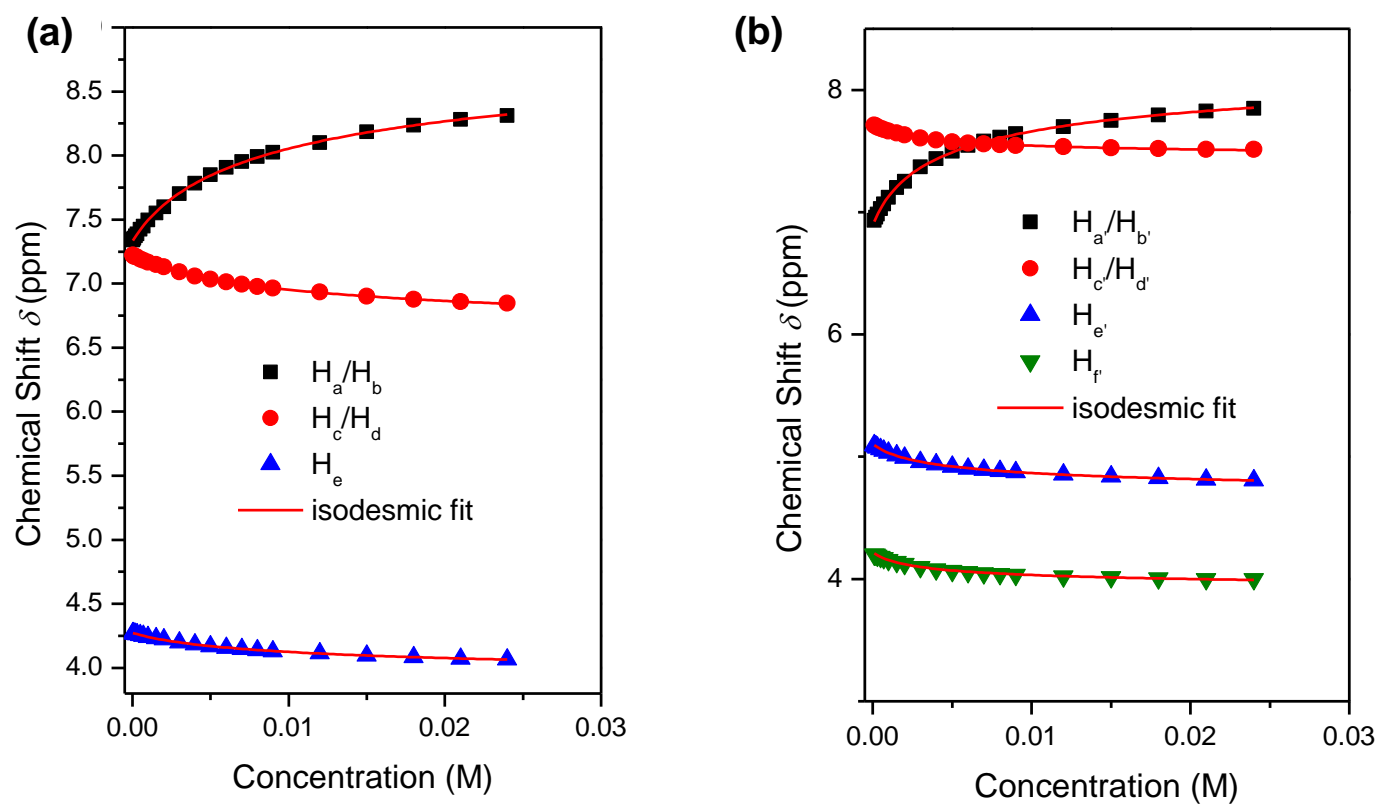

Figure S14. Concentration dependent change in chemical shift for important resonances in ( \pm )-1a from Figure S4 (a) and in ( \pm )2a from Figure S5 (b). 


\section{Infrared Spectroscopy}

Solution phase IR spectra were collected using a PerkinElmer SpectrumOne with a sealed $1 \mathrm{~mm}$ pathlength $\mathrm{CaF}_{2}$ solution cell (PIKE Technologies). Solutions were prepared using HPLC grade chloroform and methylcyclohexane purchased from Sigma-Aldrich. Solid-state IR spectra were collected using using a PerkinElmer SpectrumOne equipped with a GladiATR ${ }^{\text {TM }}$ attachment (PIKE Technologies). Solid samples were dried under vacuum prior to measurement. All measurements were taken using PerkinElmer Spectrum Software.

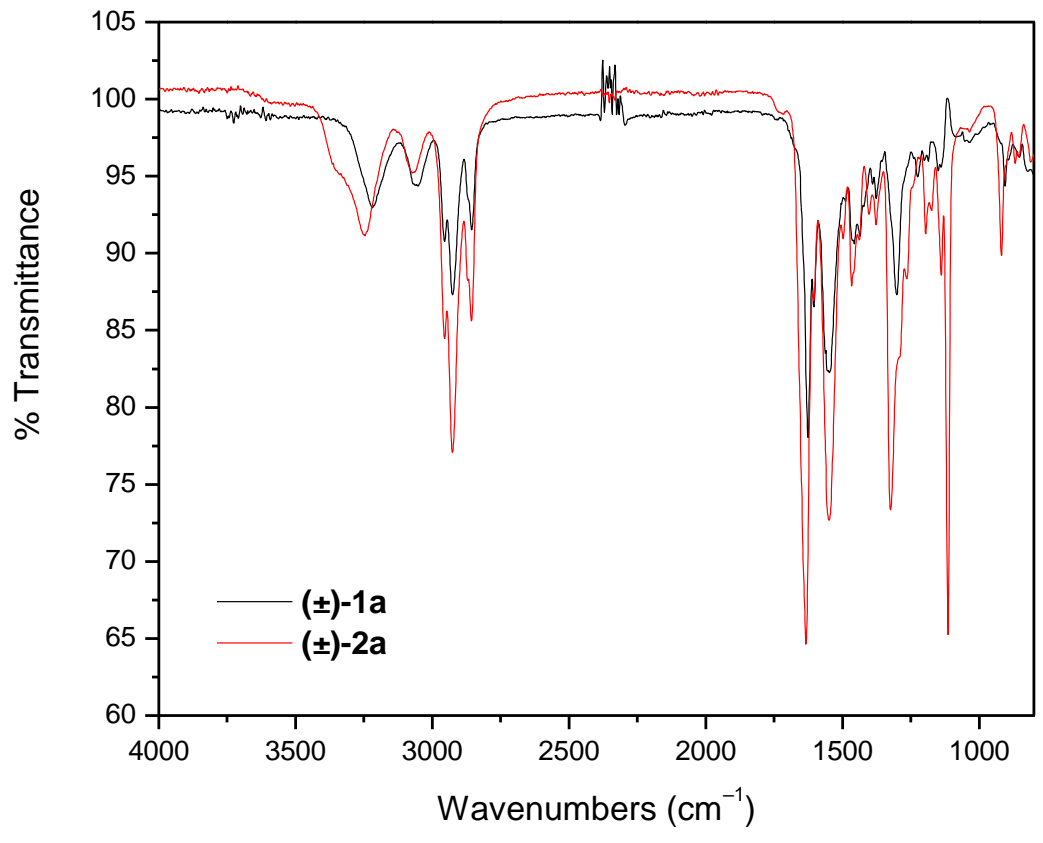

Figure S15. Overlaid FT-IR (ATR) of ( \pm )-1a and $( \pm)-2 a$ in the solid state. 


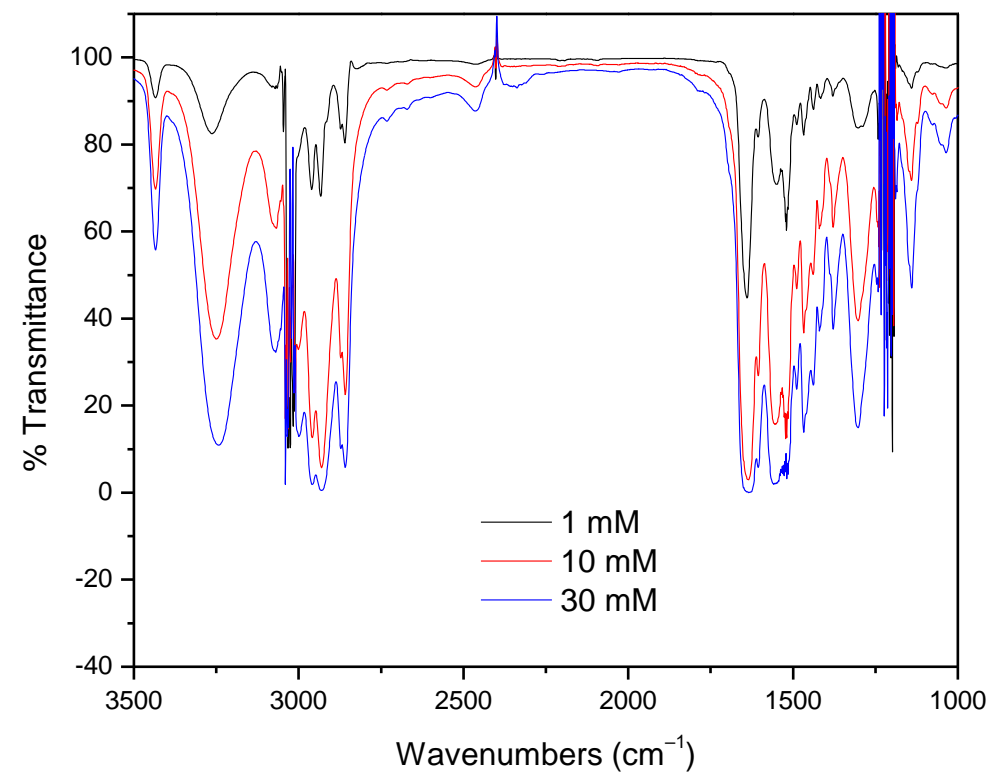

Figure S16. Overlaid IR spectra of ( \pm -1a in $\mathrm{CHCl}_{3}$ from $30-1 \mathrm{mM}$ at $298 \mathrm{~K}$.

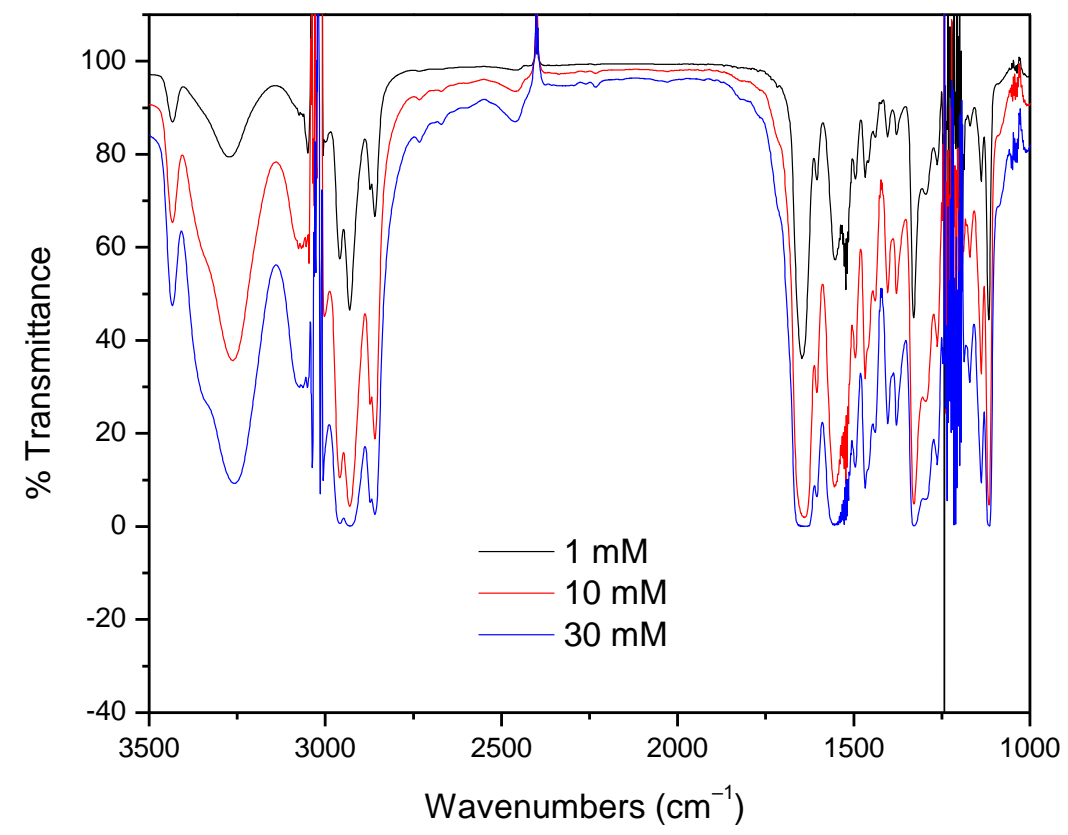

Figure S17. Overlaid FT-IR spectra of ( \pm -2a in $\mathrm{CHCl}_{3}$ from $30-1 \mathrm{mM}$ at $298 \mathrm{~K}$. 


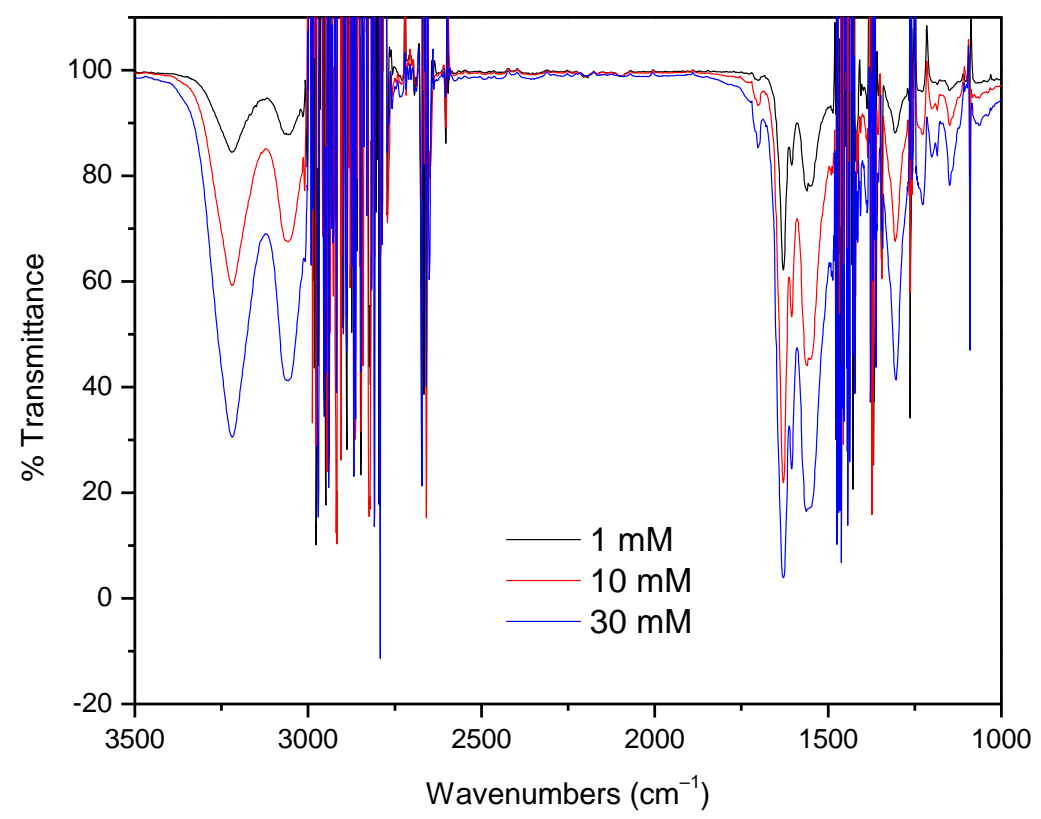

Figure S18. Overlaid IR spectra of ( \pm )-1a in MCH from 30-1 mM at $298 \mathrm{~K}$.

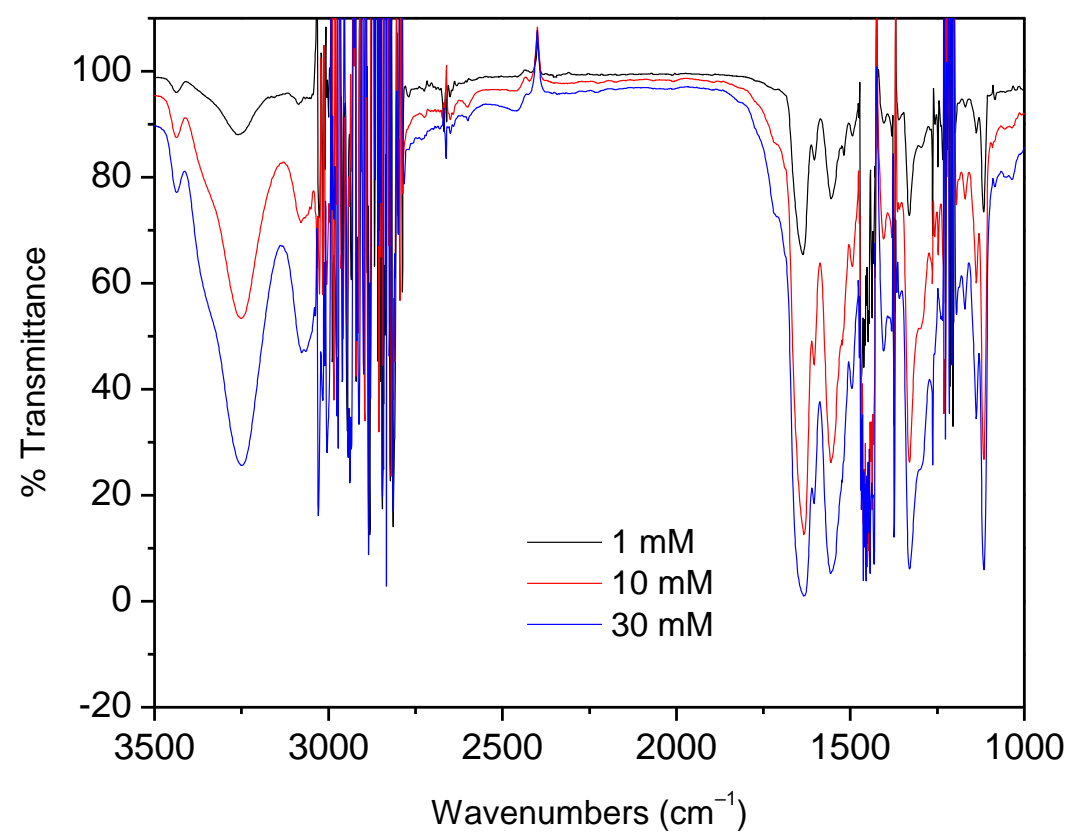

Figure S19. Overlaid IR spectra of ( \pm -2a in $\mathrm{CHCl}_{3} / \mathrm{MCH}(1: 1 \mathrm{v} / \mathrm{v})$ from $30-1 \mathrm{mM}$ at $298 \mathrm{~K}$. 


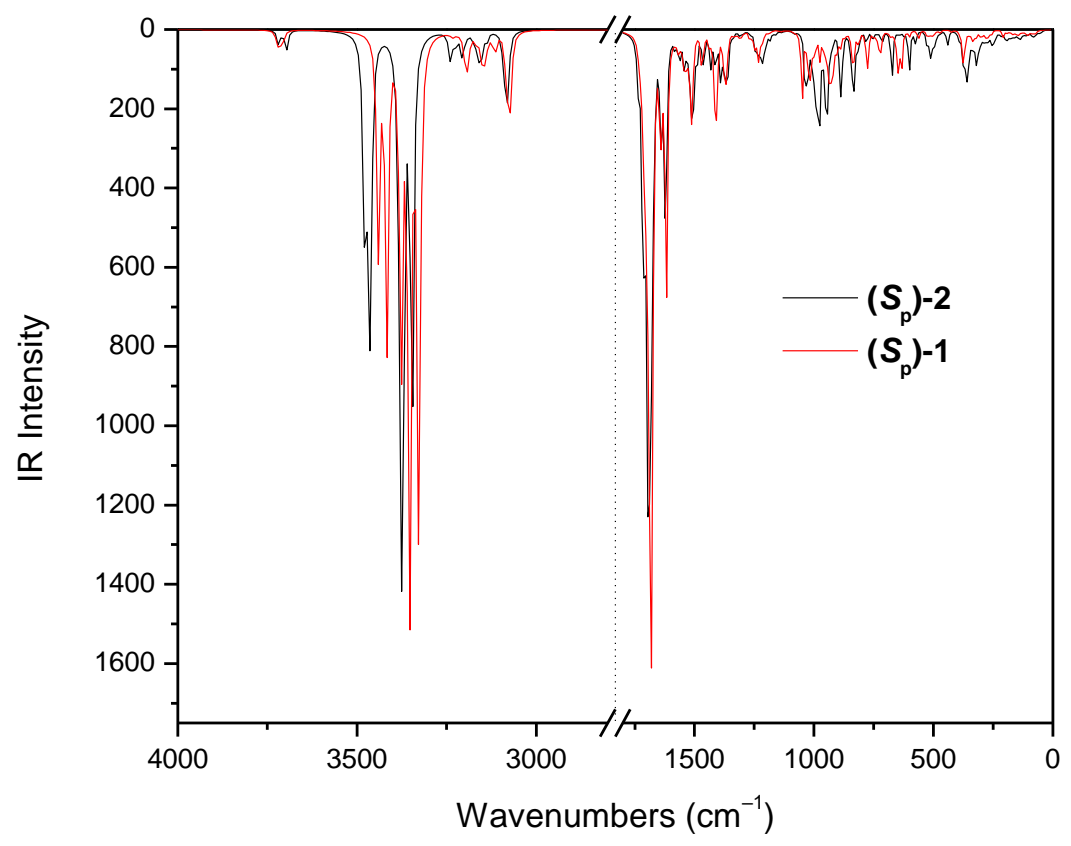

Figure S20. Overlaid calculated IR spectra of $\left(\boldsymbol{S}_{\mathbf{p}}\right)-\mathbf{1}$ and $\left(\boldsymbol{S}_{\mathrm{p}}\right)-\mathbf{2}$ dimers. 
Table S3. Selected peaks of compounds ( \pm )-1a and ( \pm )-2a from solution-phase FT-IR spectroscopy and the solid-state IR (ATR) spectroscopy.

\begin{tabular}{|c|c|c|c|c|c|}
\hline Compound & Solvent & Concentration & NH (solvated) & NH (H-bonded) & $\mathrm{C}=\mathrm{O}$ \\
\hline$( \pm)-1 a$ & $\mathrm{CHCl}_{3}$ & $1 \mathrm{mM}$ & 3434 & 3263 & 1637 \\
\hline$( \pm)-1 a$ & $\mathrm{CHCl}_{3}$ & $10 \mathrm{mM}$ & 3434 & 3250 & 1635 \\
\hline$( \pm)-1 a$ & $\mathrm{CHCl}_{3}$ & $30 \mathrm{mM}$ & 3434 & 3243 & 1635 \\
\hline$( \pm)-1 \mathrm{a}$ & $\mathrm{MCH}$ & $1 \mathrm{mM}$ & - & 3222 & 1629 \\
\hline$( \pm)-1 a$ & $\mathrm{MCH}$ & $10 \mathrm{mM}$ & - & 3219 & 1629 \\
\hline$( \pm)-1 a$ & $\mathrm{MCH}$ & $30 \mathrm{mM}$ & - & 3219 & 1629 \\
\hline$( \pm)-1 a$ & Solid & - & - & 3219 & 1626 \\
\hline$( \pm)-2 a$ & $\mathrm{CHCl}_{3}$ & $1 \mathrm{mM}$ & 3434 & 3273 & 1647 \\
\hline$( \pm)-2 a$ & $\mathrm{CHCl}_{3}$ & $10 \mathrm{mM}$ & 3434 & 3262 & 1639 \\
\hline$( \pm)-2 a$ & $\mathrm{CHCl}_{3}$ & $30 \mathrm{mM}$ & 3434 & 3258 & $1630-1640$ \\
\hline$( \pm)-2 a$ & Solid & - & - & 3243 & 1633 \\
\hline
\end{tabular}

Table S4. Calculated N-H stretching frequencies for dimers of $\left(S_{\mathbf{p}}\right)-\mathbf{1}$ and $\left(\boldsymbol{S}_{\mathbf{p}}\right)-\mathbf{2}$ in the chair anti- conformation. NH types: 1 intermolecular H-bond, no $n \rightarrow \pi^{*} ; \mathbf{2}$ intermolecular H-bond, $n \rightarrow \pi^{*} ; \mathbf{3}$ transannular H-bond, no $n \rightarrow \pi^{*} ; \mathbf{4}$ transannular H-bond, $n \rightarrow \pi^{*} ; \mathbf{5}$ no H-bond, no $n \rightarrow \pi^{*} ; \mathbf{6}$ no H-bond, $n \rightarrow \pi^{*}$.

\begin{tabular}{cccc}
\hline $\mathbf{1 ~ N H}\left(\mathbf{c m}^{-\mathbf{1}}\right)$ & type & $\mathbf{2 ~ N H}\left(\mathbf{c m}^{-\mathbf{1}}\right)$ & type \\
\hline 3326.20 & 1 & 3345.07 & 3 \\
3352.12 & 3 & 3370.03 & 3 \\
3375.88 & 3 & 3376.43 & 1 \\
3415.3 & 2 & 3460.74 & 4 \\
3416.48 & 4 & 3465.96 & 2 \\
3439.43 & 4 & 3477.35 & 4 \\
3708.05 & 5 & 3696.52 & 5 \\
3721.05 & 6 & 3717.67 & 6 \\
\hline
\end{tabular}




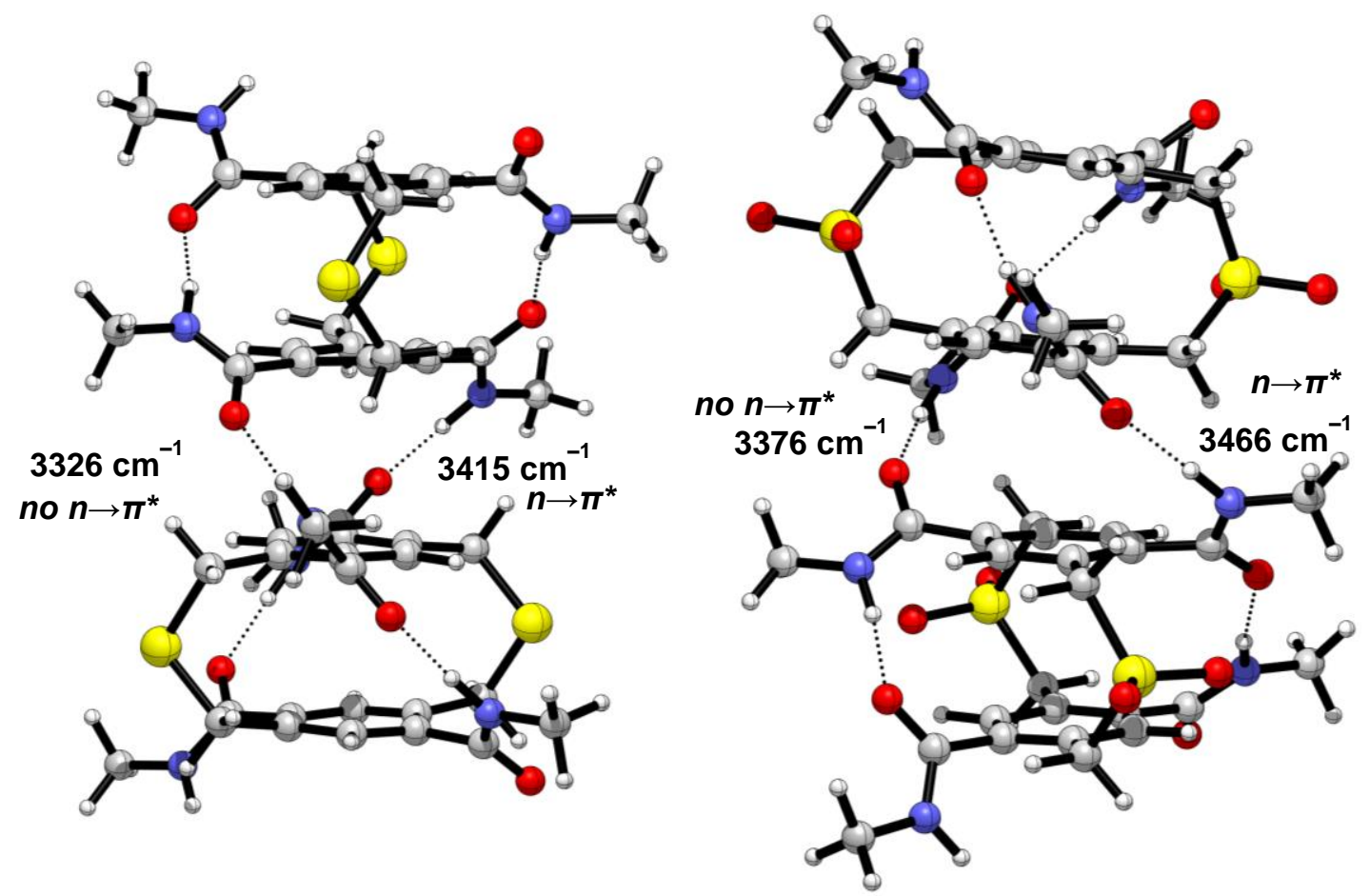

Figure S21. Differences in N-H stretching frequencies calculated for $\left(\boldsymbol{S}_{\mathbf{p}}\right)$-1 (left) and $\left(\boldsymbol{S}_{\mathbf{p}}\right)$-2 (right) dimers calculated at the $\omega B 97 X-D / 6-31 G$ level of theory. 


\section{UV-Vis Spectroscopy}

Solutions of ( \pm )-1a and ( \pm )-2a in $\mathrm{CHCl}_{3}$ were prepared and absorbance measurements were acquired using a Cary 100 Bio UV-Visible dual beam spectrophotometer controlled by Cary Win UV software. Time-dependent DFT calculations at the $\omega$ B97X-D/6-31G level of theory were used to obtain simulated UV-Vis spectra at 10 points. The simulated spectra were processed with GaussSum 3.1. ${ }^{8}$

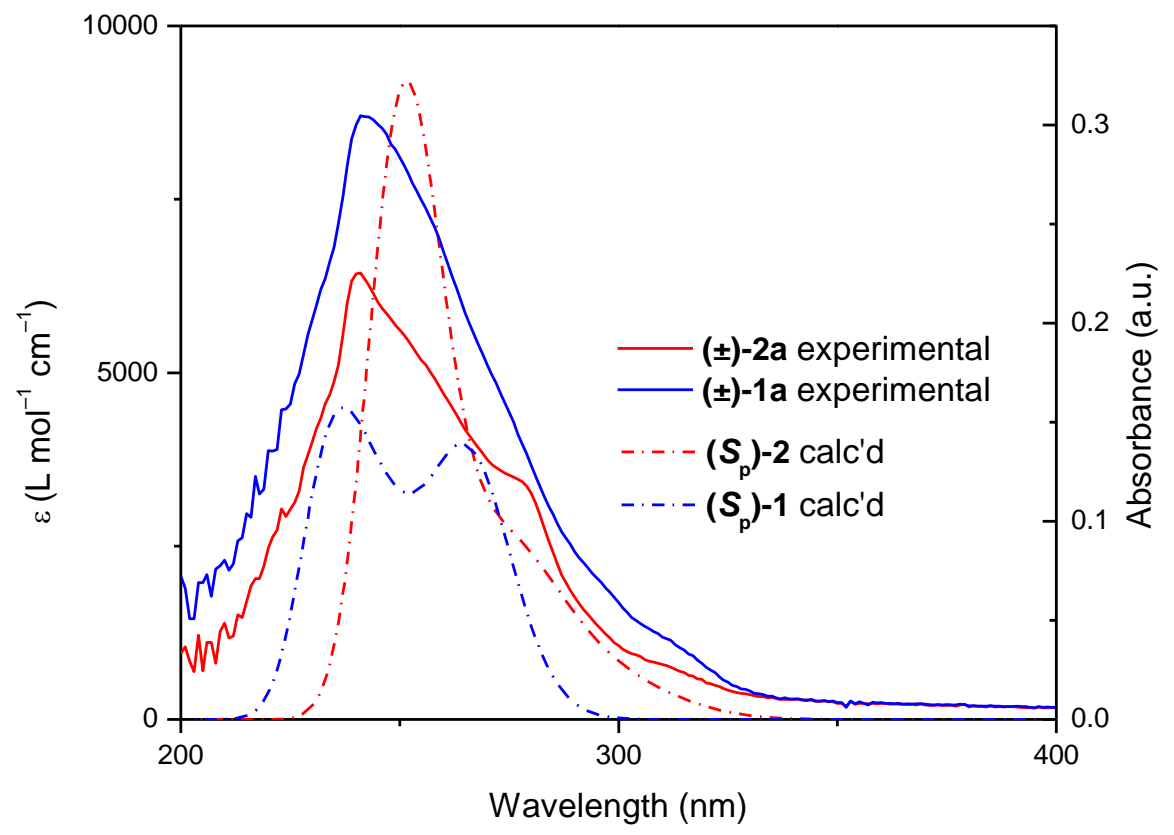

Figure S22. Overlaid experimental UV-Vis spectra of $( \pm)-\mathbf{1}$ and $( \pm)-2\left(20 \mu \mathrm{M}\right.$ in $\left.\mathrm{CHCl}_{3}\right)$ and calculated $\mathrm{UV}$-Vis spectra of $\left(\boldsymbol{S}_{\mathbf{p}}\right)-\mathbf{1}$ and $\left(\boldsymbol{S}_{\mathbf{p}}\right)-2$. 


\section{X-Ray Crystallography: \\ Experimental Details for ( \pm )-1b CCDC 2086347:}

X-Ray Intensity data were collected at $100 \mathrm{~K}$ on a Bruker DV8 Venture diffractometer using MoK $\alpha$ radiation $(\lambda=0.71073 \AA)$ and a Photon III area detector.

Raw data frames were read by program $\mathrm{SAINT}^{1}$ and integrated using $3 \mathrm{D}$ profiling algorithms. The resulting data were reduced to produce hkl reflections and their intensities and estimated standard deviations. The data were corrected for Lorentz and polarization effects and numerical absorption corrections were applied based on indexed and measured faces.

The structure was solved and refined in SHELXTL2014, ${ }^{9}$ using full-matrix least-squares refinement. The non- $\mathrm{H}$ atoms were refined with anisotropic thermal parameters and all of the $\mathrm{H}$ atoms were calculated in idealized positions and refined riding on their parent atoms. The asymmetric unit consists of two chemically equivalent but crystallographically independent molecules. Each molecule has several disordered regions. Molecule A has all phenyl rings disordered and each one is refined in two parts. Molecule B has only one disordered phenyl ring which was similarly refined. The Sulfur bridging atoms are disordered and each was refined in two parts. In the final cycle of refinement, 18804 reflections (of which 15333 are observed with $\mathrm{I}>2 \sigma(\mathrm{I})$ ) were used to refine 1175 parameters and the resulting $\mathrm{R}_{1}$, wR 2 and S (goodness of fit) were $5.02 \%, 12.38 \%$ and 1.051, respectively. The refinement was carried out by minimizing the $w R_{2}$ function using $F^{2}$ rather than $F$ values. $R_{1}$ is calculated to provide a reference to the conventional $\mathrm{R}$ value but its function is not minimized. 
Table 5. Crystal data and structure refinement for ( \pm )-1b.

Identification code when17

Empirical formula

C48 H44 N4 O4 S2

Formula weight

804.99

Temperature $100(2) \mathrm{K}$

Wavelength $0.71073 \AA$

Crystal system Monoclinic

Space group P 21/c

Unit cell dimensions $\quad \mathrm{a}=11.9306(5) \AA \quad \mathrm{a}=90^{\circ}$.

$$
\begin{array}{ll}
\mathrm{b}=29.6014(13) \AA & \mathrm{b}=103.098(2)^{\circ} . \\
\mathrm{c}=23.7710(11) \AA & \mathrm{g}=90^{\circ} .
\end{array}
$$

Volume8176.6(6) ^̊3

Z 8

Density (calculated) $\quad 1.308 \mathrm{Mg} / \mathrm{m} 3$

Absorption coefficient $0.181 \mathrm{~mm}-1$

$\mathrm{F}(000) 3392$

Crystal size $\quad 0.300 \times 0.144 \times 0.060 \mathrm{~mm} 3$

Theta range for data collection 1.883 to $27.500^{\circ}$.

Index ranges $\quad-15 \leq \mathrm{h} \leq 15,-38 \leq \mathrm{k} \leq 38,-30 \leq \mathrm{l} \leq 30$

Reflections collected 193943

Independent reflections 18804 [R(int $)=0.0492]$

Completeness to theta $=25.000^{\circ} 99.9 \%$

Absorption correction "multi-scan"

Max. and min. transmission $\quad 0.9912$ and 0.9726

Refinement method Full-matrix least-squares on F2

Data / restraints / parameters 18804 / 1156/1175

Goodness-of-fit on F2 1.051

Final $\mathrm{R}$ indices $[\mathrm{I}>2 \operatorname{sigma}(\mathrm{I})] \quad \mathrm{R} 1=0.0502, \mathrm{wR} 2=0.1238$ [15333]

$\mathrm{R}$ indices (all data) $\mathrm{R} 1=0.0643, \mathrm{wR} 2=0.1332$

Largest diff. peak and hole $\quad 0.669$ and -0.316 e. $\AA$ - 3

$\mathrm{R} 1=\mathrm{a}(|| \mathrm{Fo}|-| \mathrm{Fc} \|) / \mathrm{a}|\mathrm{Fo}| \quad \mathrm{wR} 2=[\mathrm{a}[\mathrm{w}(\mathrm{Fo} 2-\mathrm{Fc} 2) 2] / \stackrel{\mathrm{a}}{\mathrm{w}}[\mathrm{w}(\mathrm{Fo} 2) 2]] 1 / 2$

$\mathrm{S}=[\mathrm{a}[\mathrm{w}(\mathrm{Fo} 2-\mathrm{Fc} 2) 2] /(\mathrm{n}-\mathrm{p})] 1 / 2 \quad \mathrm{w}=1 /\left[\mathrm{s} 2(\mathrm{Fo} 2)+(\mathrm{m} * \mathrm{p})^{2}+\mathrm{n}^{*} \mathrm{p}\right], \mathrm{p}=[\max (\mathrm{Fo} 2,0)+2 * \mathrm{Fc} 2] / 3, \mathrm{~m} \&$ $\mathrm{n}$ are constants. 


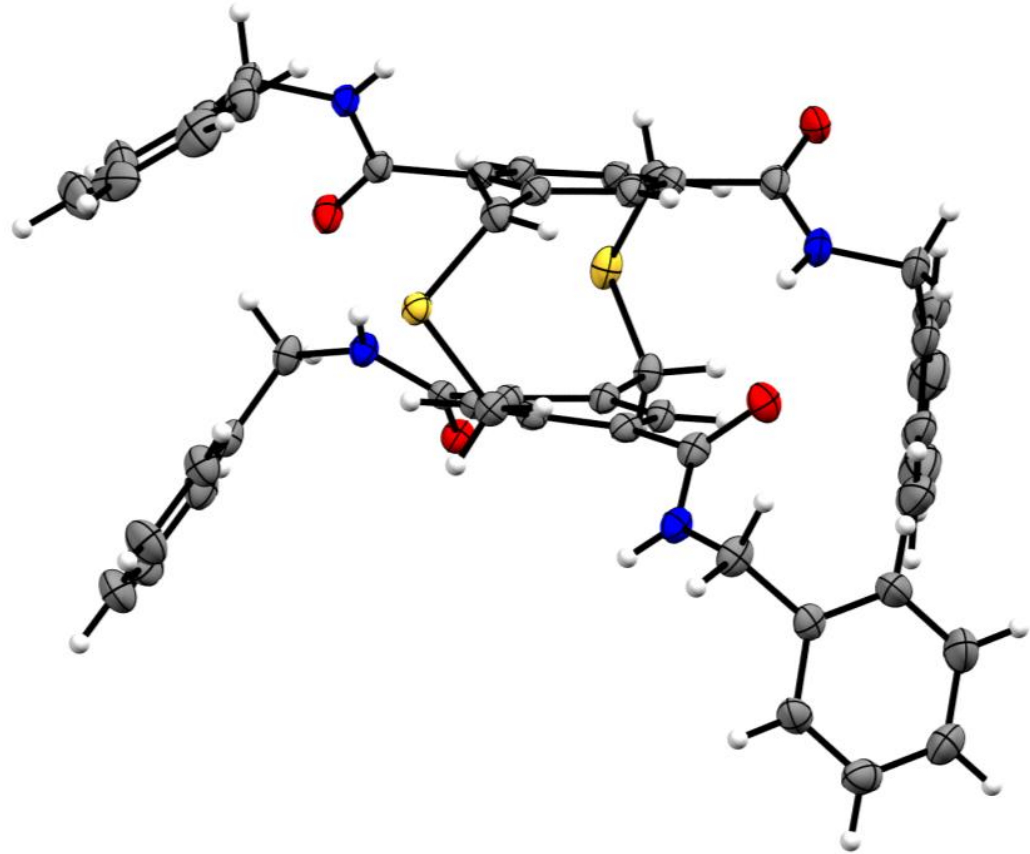

Figure S23. ORTEP of $\left(\boldsymbol{R}_{\mathbf{p}}\right)$-1a shown in the chair conformation. Bridge disorder and hydrogen atoms omitted for clarity. Ellipsoids shown at $50 \%$ probability. Atom color code: O (red), N (blue), C (gray), S (yellow).

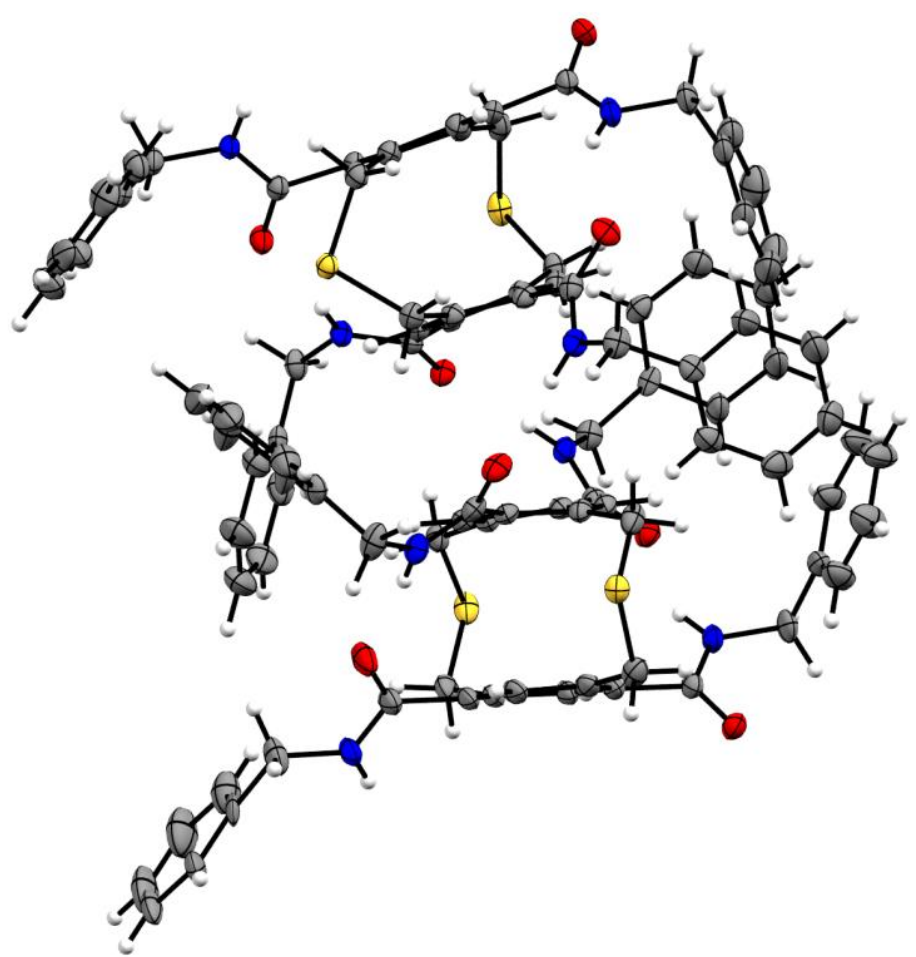

Figure S24. ORTEP of the asymmetric unit of $( \pm)$-1b showing a dimer of $\left(\boldsymbol{R}_{\mathbf{p}}\right)$-1b. Disorder omitted for clarity. Ellipsoids shown at $50 \%$ probability. Atom color code: $\mathrm{O}$ (red), N (blue), C (gray), S (yellow). 


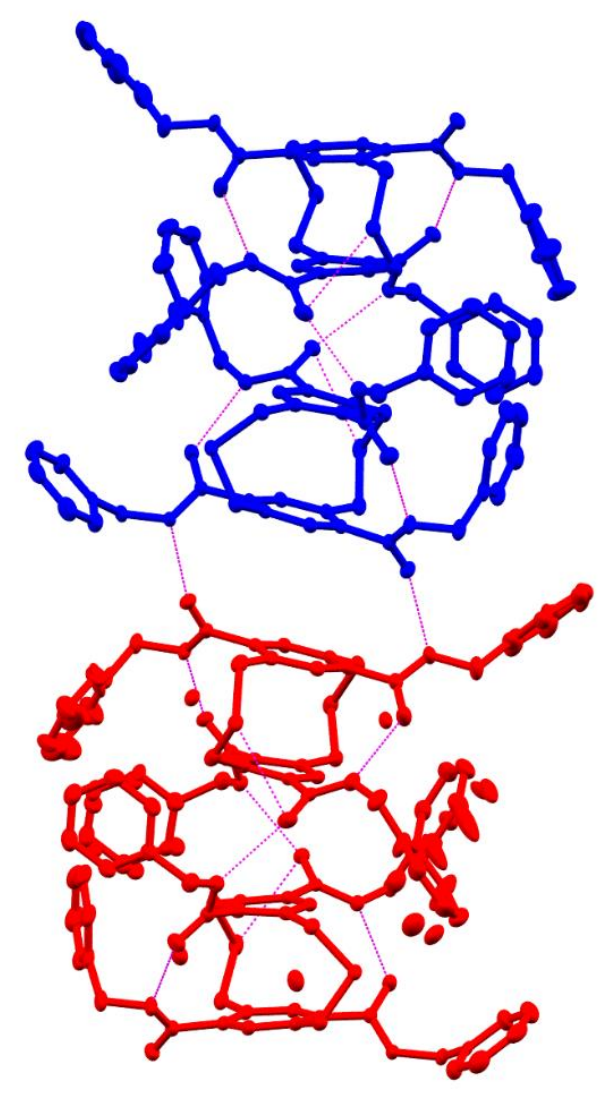

Figure S25. Columnar packing of $\left(\boldsymbol{S}_{\mathbf{p}}\right)$-1b (blue) and $\left(\boldsymbol{R}_{\mathbf{p}}\right)$-1b (red). Hydrogen bonds shown in magenta, hydrogen atoms omitted for clarity. Ellipsoids shown at $50 \%$ probability.

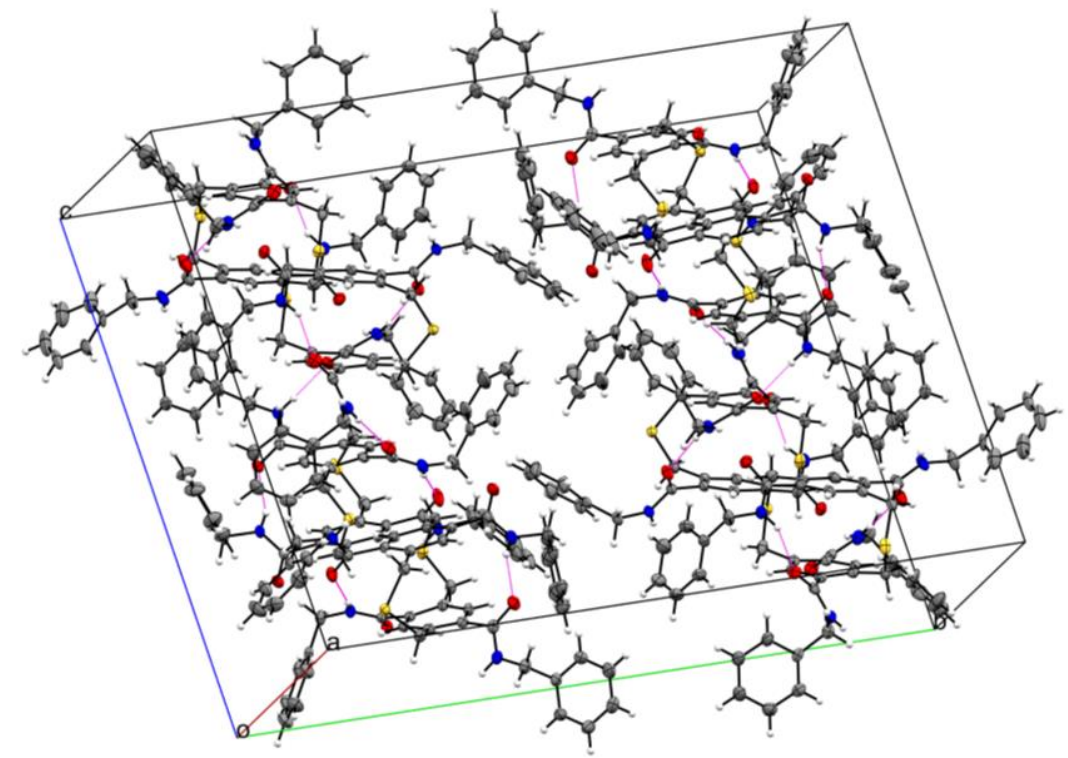

Figure S26. Unit cell of ( \pm )-1b viewed orthogonal to the assembly axis. Disorder omitted for clarity. Atom color code: O (red), $\mathrm{N}$ (blue), C (gray), S (yellow). Ellipsoids shown at $50 \%$ probability. 


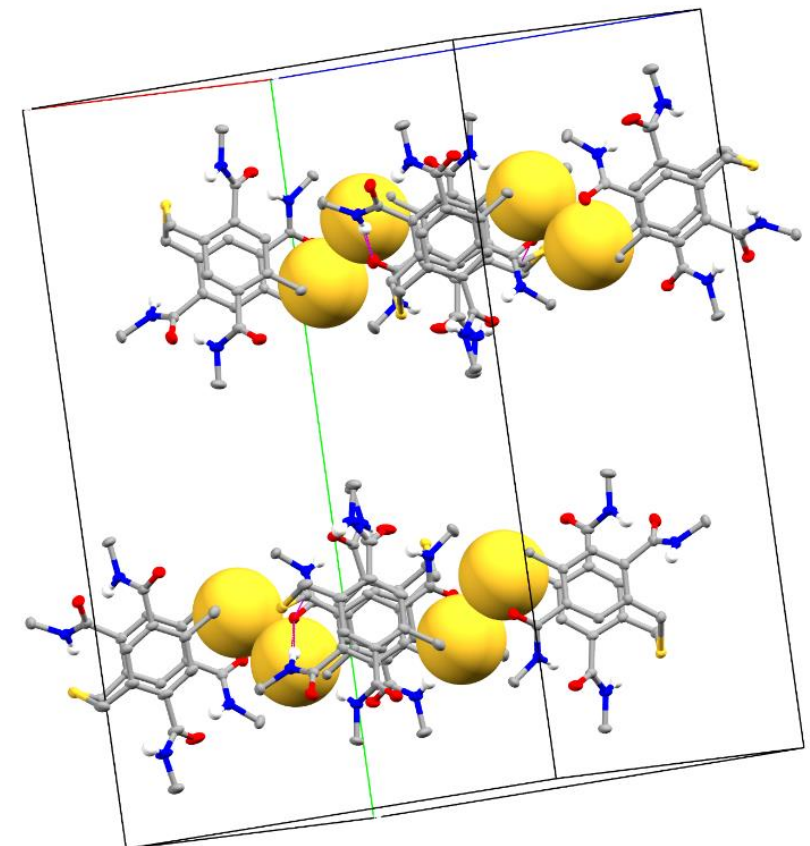

Figure S27. View of the unit cell of ( \pm )-1b from above the assembly axis showing intercolumnar $S \cdots S$ interactions. Sulfur atoms shown as enlarged spheres, Benzyl sidechain and bridge sulfur disorder omitted for clarity.

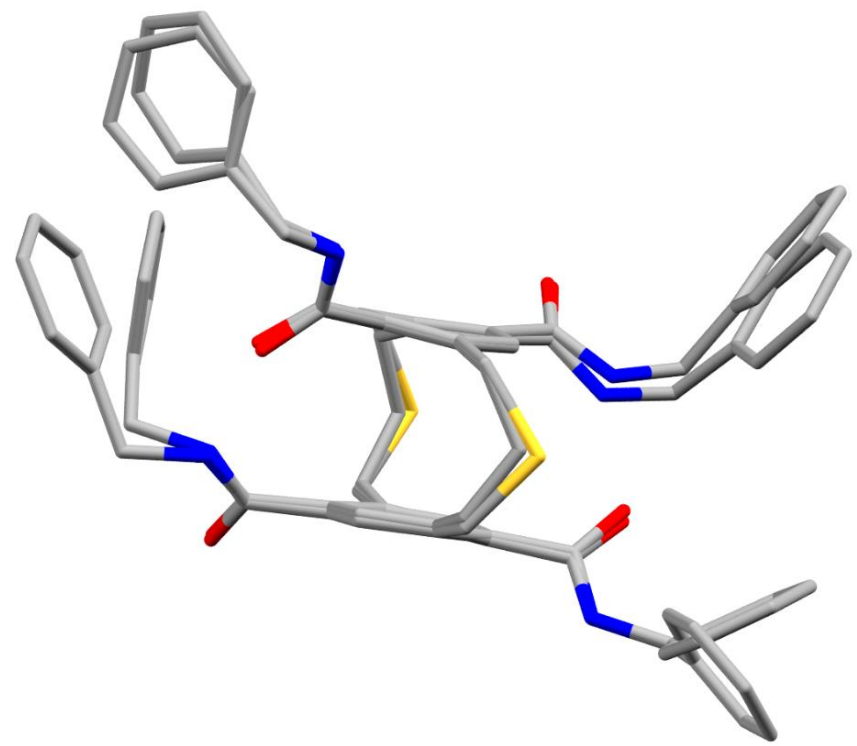

Figure S28. Overlaid X-ray structures of $\left(\boldsymbol{R}_{\mathbf{p}}\right)$-1a and $\left(\boldsymbol{R}_{\mathbf{p}}\right)-[3.3]$ pCpTA-benzyl (CCDC Code 1949727). 


\section{Experimental Details for ( \pm )-2c CCDC 2086349:}

X-Ray Intensity data were collected at $100 \mathrm{~K}$ on a Bruker Dual micro source D8 Venture diffractometer and PHOTON III detector running APEX3 software package of programs and using MoK $\alpha$ radiation $(\lambda$ $=0.71073 \AA$ ).

The data frames were integrated and multi-scan scaling was applied in APEX3. Intrinsic phasing structure solution provided all of the non-H atoms.

The structure was refined using full-matrix least-squares refinement. ${ }^{9}$ The non- $\mathrm{H}$ atoms were refined with anisotropic displacement parameters and all of the $\mathrm{H}$ atoms were calculated in idealized positions and refined riding on their parent atoms. In addition to the molecule, there is a dimethylformamide solvent molecule, and four water molecules. The water protons were obtained from a Difference Fourier map and refined riding on their oxygen atoms. All of the acidic protons are involved in hydrogen bonding which are tabulated in the hydrogen bonding table. In the final cycle of refinement, 9447 reflections (of which 8335 are observed with $\mathrm{I}>2 \sigma(\mathrm{I}))$ were used to refine 528 parameters and the resulting $\mathrm{R}_{1}, \mathrm{wR}_{2}$ and $\mathrm{S}$ (goodness of fit) were $5.89 \%, 17.06 \%$ and 1.072, respectively. The refinement was carried out by minimizing the $w R_{2}$ function using $F^{2}$ rather than $F$ values. $R_{1}$ is calculated to provide a reference to the conventional $\mathrm{R}$ value but its function is not minimized. 
Table 6. Crystal data and structure refinement for $( \pm)-2 c$.

Identification code

Empirical formula

Formula weight

Temperature

Wavelength

Crystal system

Space group

Unit cell dimensions

Volume

Z

Density (calculated)

Absorption coefficient

$\mathrm{F}(000)$

Crystal size

Theta range for data collection

Index ranges

Reflections collected

Independent reflections

Completeness to theta $=25.242^{\circ}$

Absorption correction

Refinement method

Data / restraints / parameters

Goodness-of-fit on $\mathrm{F}^{2}$

Final $R$ indices [I>2sigma(I)]

$\mathrm{R}$ indices (all data)

Extinction coefficient

Largest diff. peak and hole

$\mathrm{R} 1=\sum\left(|| \mathrm{F}_{\mathrm{O}}|-| \mathrm{F}_{\mathrm{C}} \|\right) / \sum\left|\mathrm{F}_{\mathrm{O}}\right|$

$\mathrm{S}=\left[\sum\left[\mathrm{w}\left(\mathrm{F}_{\mathrm{O}}^{2}-\mathrm{F}_{\mathrm{c}}^{2}\right)^{2}\right] /(\mathrm{n}-\mathrm{p})\right]^{1 / 2}$ when 20

C32 H44 N4 O8 S2

676.83

100(2) K

$0.71073 \AA$

Monoclinic

$\mathrm{P} 21 / \mathrm{c}$

$\mathrm{a}=9.3413(3) \AA \quad \alpha=90^{\circ}$.

$\mathrm{b}=17.8000(6) \AA \quad \beta=92.5620(10)^{\circ}$.

$\mathrm{c}=24.8065(8) \AA \quad \gamma=90^{\circ}$.

4120.6(2) $\AA^{3}$

4

$1.091 \mathrm{Mg} / \mathrm{m}^{3}$

$0.175 \mathrm{~mm}^{-1}$

1440

$0.329 \times 0.165 \times 0.118 \mathrm{~mm}^{3}$

2.003 to $27.499^{\circ}$.

$-12 \leq \mathrm{h} \leq 12,-23 \leq \mathrm{k} \leq 23,-32 \leq \mathrm{l} \leq 32$

128715

$9447[\mathrm{R}($ int $)=0.2028]$

$99.9 \%$

None

Full-matrix least-squares on $\mathrm{F}^{2}$

9447 / 0 / 528

1.072

$\mathrm{R} 1=0.0589, \mathrm{wR} 2=0.1706$ [8335]

$\mathrm{R} 1=0.0633, \mathrm{wR} 2=0.1749$

n/a

1.298 and -0.771 e. $\AA^{-3}$

$w R 2=\left[\sum\left[w\left(F_{o}^{2}-F_{c}^{2}\right)^{2}\right] / \sum\left[w\left(F_{o}{ }^{2}\right)^{2}\right]\right]^{1 / 2}$

$\mathrm{w}=1 /\left[\sigma^{2}\left(\mathrm{~F}_{\mathrm{O}}{ }^{2}\right)+(\mathrm{m} * \mathrm{p})^{2}+\mathrm{n} * \mathrm{p}\right], \mathrm{p}=\left[\max \left(\mathrm{F}_{\mathrm{O}}{ }^{2}, 0\right)+2 * \mathrm{~F}_{\mathrm{c}}{ }^{2}\right] / 3, \mathrm{~m} \& \mathrm{n}$ are constants. 


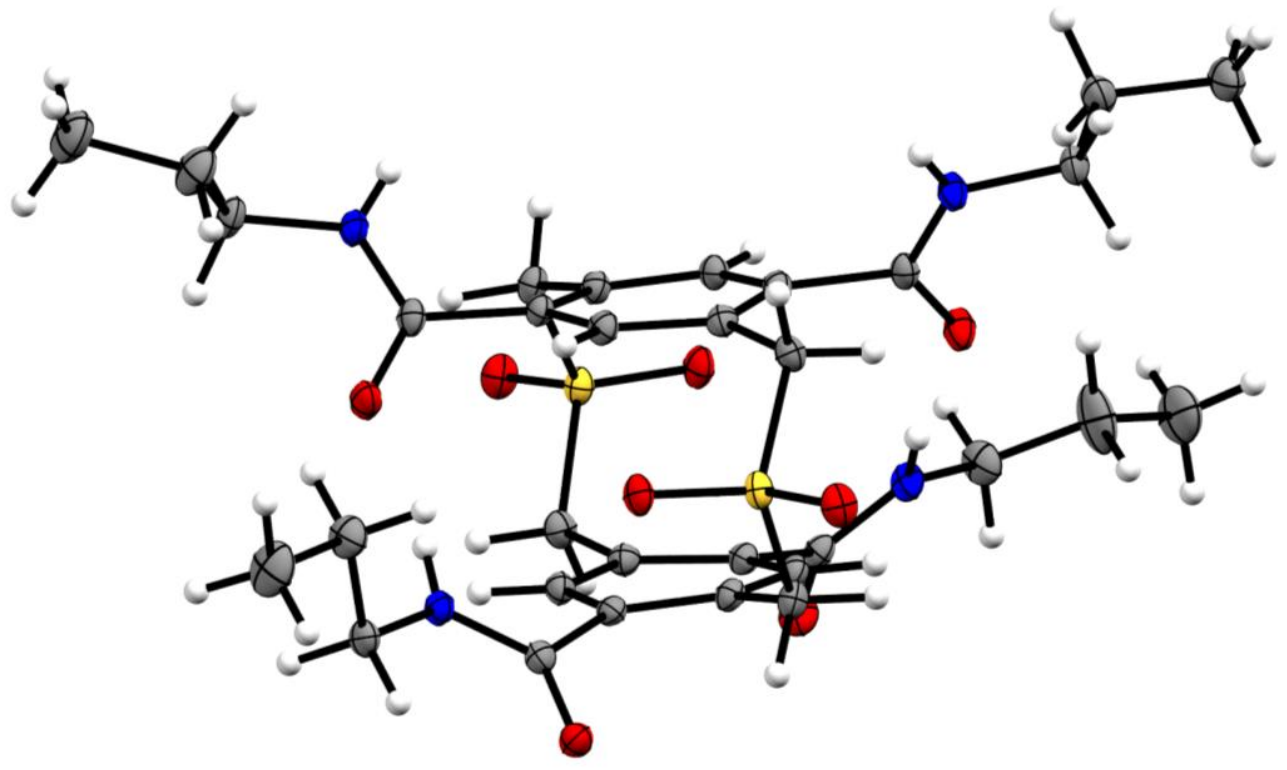

Figure S29. ORTEP of $\left(S_{\mathbf{p}}\right)$-2c from X-ray crystallography with ellipsoids shown at $50 \%$ probability. Atom color code: O (red), $\mathrm{N}$ (blue), $\mathrm{C}$ (gray), $\mathrm{S}$ (yellow) $\mathrm{H}$ (white).

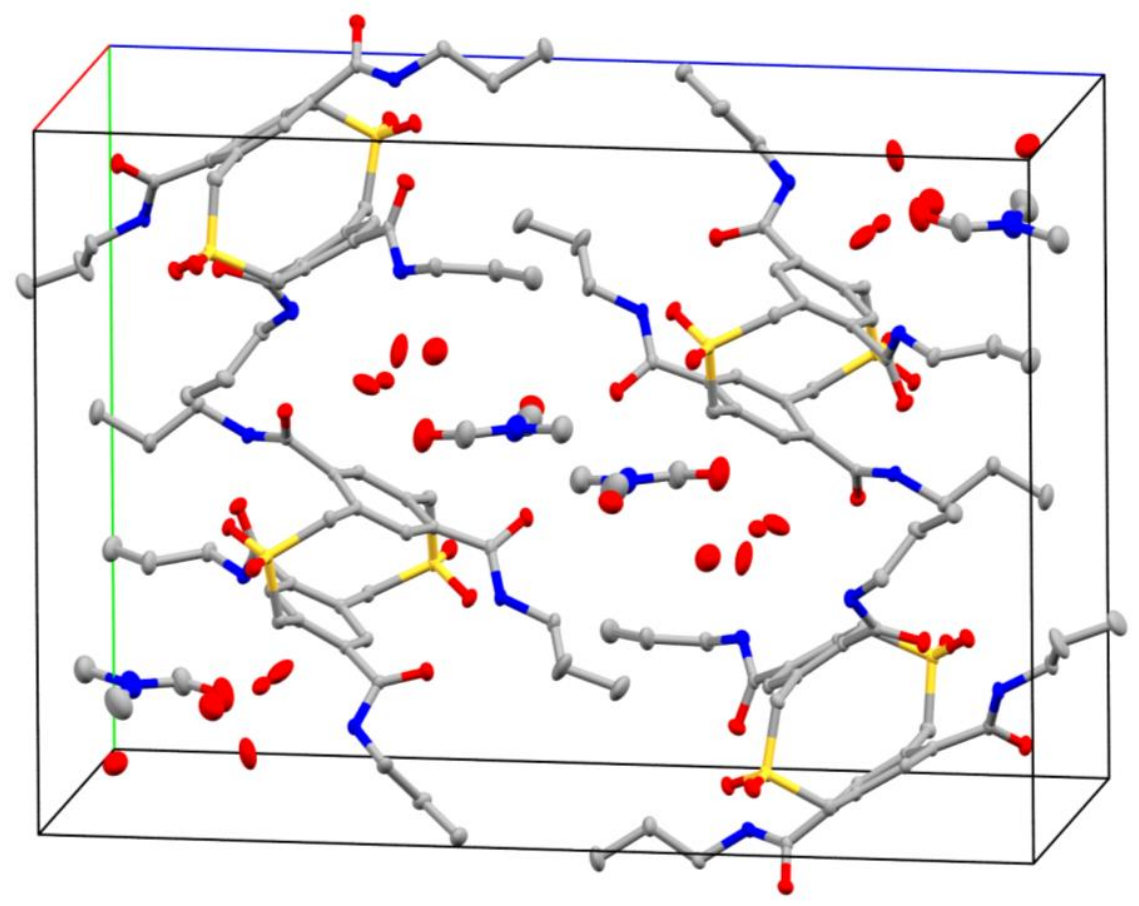

Figure S30. Unit cell of ( \pm -2c from X-ray crystallography with ellipsoids shown at 50\% probability. Hydrogen atoms omitted for clarity. 


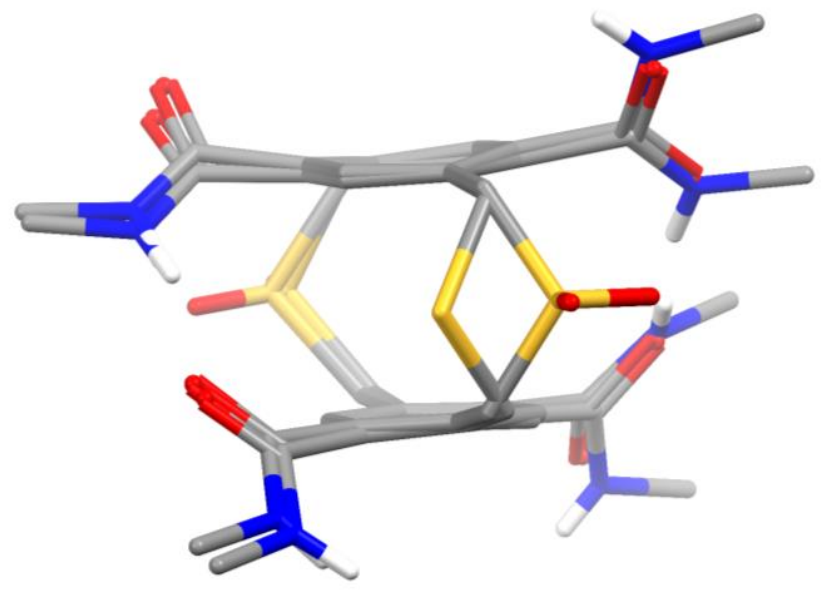

Figure S31. Overlaid structures of $\left(S_{\mathrm{p}}\right)$-2c and $\left(S_{\mathrm{p}}\right)$-1b from X-ray crystallography with sidechains and hydrogen atoms not involved in hydrogen bonding omitted for clarity. 


\section{Experimental Details for 3b CCDC 2086348:}

X-Ray Intensity data were collected at $100 \mathrm{~K}$ on a Bruker Dual micro source D8 Venture diffractometer and PHOTON III detector running APEX3 software package of programs and using MoK $\alpha$ radiation $(\lambda$ $=0.71073 \AA$ ).

The data frames were integrated and multi-scan scaling was applied in APEX3. Intrinsic phasing structure solution provided all of the non-H atoms.

The structure was refined using full-matrix least-squares refinement. ${ }^{9}$ The non- $\mathrm{H}$ atoms were refined with anisotropic displacement parameters and all of the $\mathrm{H}$ atoms were calculated in idealized positions and refined riding on their parent atoms. The asymmetric unit consists of two half molecules located on inversion centers. The molecules are chemically equivalent but crystallographically independent. Aside from the half molecules, One DMSO hydrogen bonded to a water molecule, and two other DMSO molecules disordered and each refined in two parts. In the final cycle of refinement, 11749 reflections (of which 10480 are observed with $\mathrm{I}>2 \sigma(\mathrm{I})$ ) were used to refine 466 parameters and the resulting $\mathrm{R}_{1}, w \mathrm{R}_{2}$ and $\mathrm{S}$ (goodness of fit) were $3.39 \%, 9.43 \%$ and 1.048 , respectively. The refinement was carried out by minimizing the $\mathrm{wR}_{2}$ function using $\mathrm{F}^{2}$ rather than $\mathrm{F}$ values. $\mathrm{R}_{1}$ is calculated to provide a reference to the conventional $\mathrm{R}$ value but its function is not minimized. 
Table S7. Crystal data and structure refinement for $\mathbf{3 b}$.

Identification code

Empirical formula

Formula weight

Temperature

Wavelength

Crystal system

Space group

Unit cell dimensions

Volume

Z

Density (calculated)

Absorption coefficient

$\mathrm{F}(000)$

Crystal size

Theta range for data collection

Index ranges

Reflections collected

Independent reflections

Completeness to theta $=25.242^{\circ}$

Absorption correction

Refinement method

Data / restraints / parameters

Goodness-of-fit on $\mathrm{F}^{2}$

Final $\mathrm{R}$ indices [I>2sigma(I)]

$\mathrm{R}$ indices (all data)

Extinction coefficient

Largest diff. peak and hole

$\mathrm{R} 1=\sum\left(|| \mathrm{F}_{\mathrm{O}}|-| \mathrm{F}_{\mathrm{C}} \|\right) / \sum\left|\mathrm{F}_{\mathrm{O}}\right|$

$\mathrm{S}=\left[\sum\left[\mathrm{w}\left(\mathrm{F}_{\mathrm{O}}{ }^{2}-\mathrm{F}_{\mathrm{c}}{ }^{2}\right)^{2}\right] /(\mathrm{n}-\mathrm{p})\right]^{1 / 2}$ when 19

C13 H18 O6 S2.50

350.42

121(2) K

$0.71073 \AA$

Triclinic

P-1

$$
\begin{array}{ll}
\mathrm{a}=10.3734(5) \AA & \alpha=80.113(2)^{\circ} . \\
\mathrm{b}=12.4398(6) \AA & \beta=84.5160(10)^{\circ} . \\
\mathrm{c}=13.0887(7) \AA & \gamma=68.2630(10)^{\circ} .
\end{array}
$$$$
\text { 1544.66(13) } \AA^{3}
$$

4

$1.507 \mathrm{Mg} / \mathrm{m}^{3}$

$0.437 \mathrm{~mm}^{-1}$

736

$0.278 \times 0.218 \times 0.072 \mathrm{~mm}^{3}$

2.115 to $33.171^{\circ}$.

$-15 \leq \mathrm{h} \leq 15,-19 \leq \mathrm{k} \leq 19,-20 \leq \mathrm{l} \leq 20$

116104

$11749[\mathrm{R}(\mathrm{int})=0.0431]$

$99.5 \%$

None

Full-matrix least-squares on $\mathrm{F}^{2}$

11749 / 776 / 466

1.048

$\mathrm{R} 1=0.0339, \mathrm{wR} 2=0.0943$ [10480]

$\mathrm{R} 1=0.0384, \mathrm{wR} 2=0.0984$

n/a

0.644 and -0.636 e. $\AA^{-3}$

$w R 2=\left[\Sigma\left[w\left(F_{o}{ }^{2}-F_{c}{ }^{2}{ }^{2}\right] / \Sigma\left[w\left(F_{o}{ }^{2}{ }^{2}\right]\right]^{1 / 2}\right.\right.$

$\mathrm{w}=1 /\left[\sigma^{2}\left(\mathrm{~F}_{\mathrm{O}}{ }^{2}\right)+(\mathrm{m} * \mathrm{p})^{2}+\mathrm{n} * \mathrm{p}\right], \mathrm{p}=\left[\max \left(\mathrm{F}_{\mathrm{O}}^{2}, 0\right)+2 * \mathrm{~F}_{\mathrm{c}}{ }^{2}\right] / 3, \mathrm{~m} \& \mathrm{n}$ are constants. 


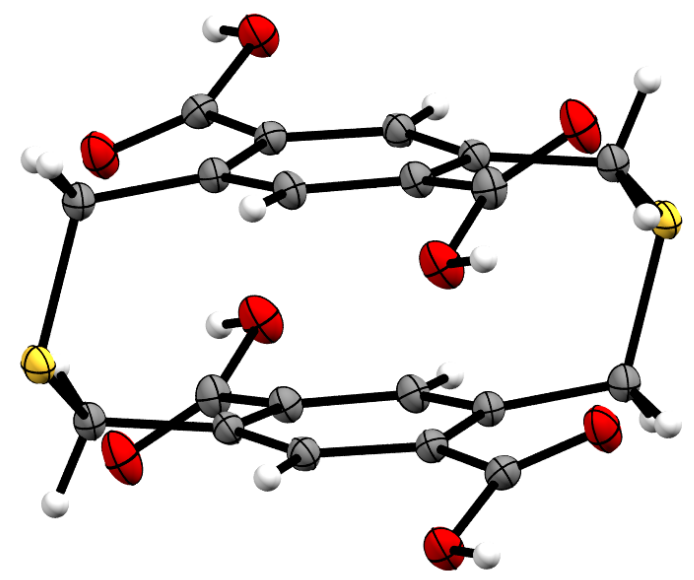

Figure S32. ORTEP of 3b from X-ray crystallography. Bridge disorder and hydrogen atoms omitted for clarity. Ellipsoids shown at $50 \%$ probability. Atom color code: $\mathrm{O}$ (red), $\mathrm{C}$ (gray), $\mathrm{S}$ (yellow), $\mathrm{H}$ (white).

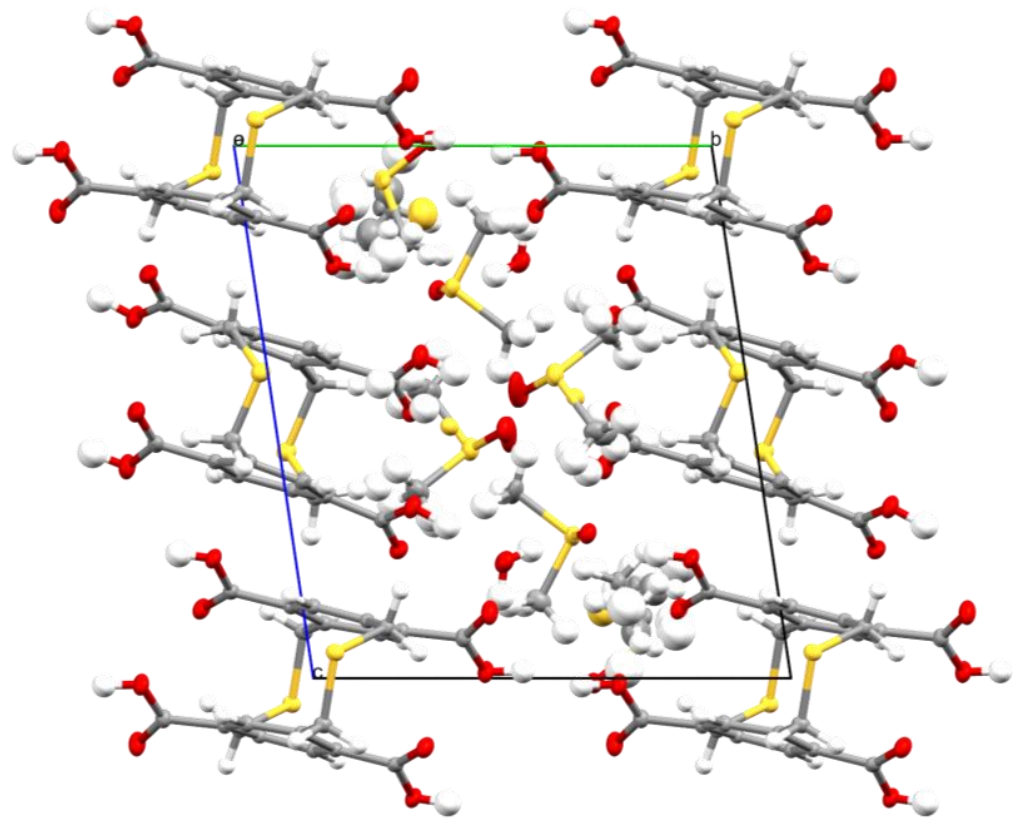

Figure S33. View of the unit cell of $\mathbf{3 b}$ viewed along the $a$-axis. 


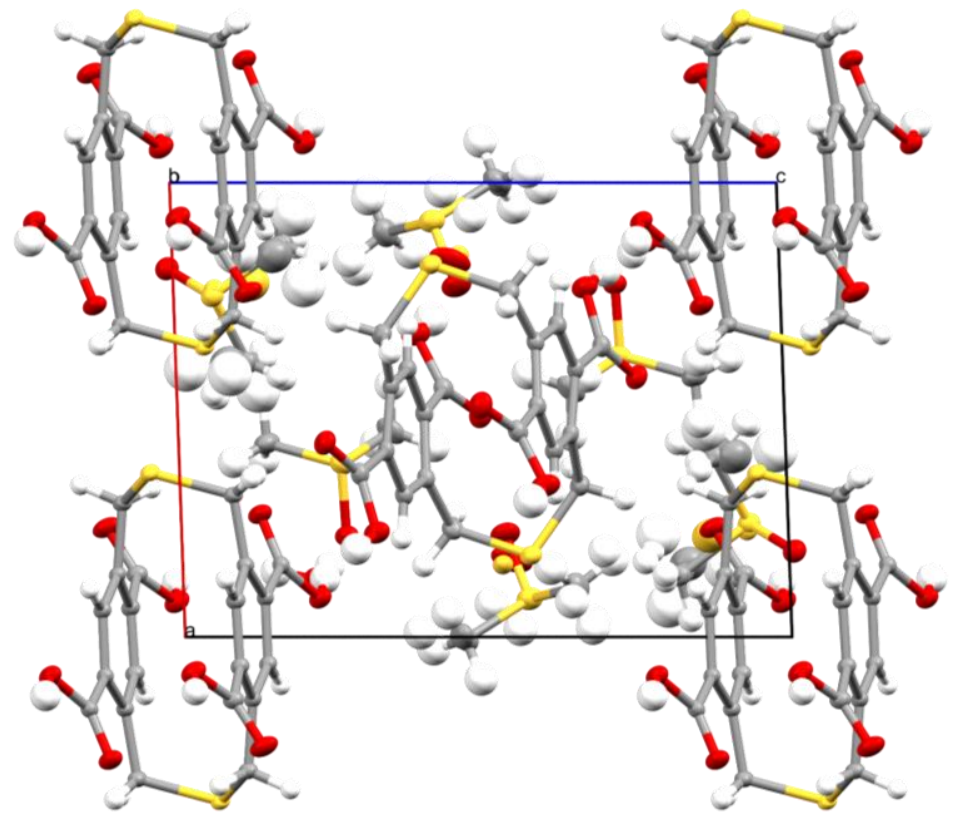

Figure S34. View of the unit cell of $\mathbf{3 b}$ viewed along the $b$-axis.

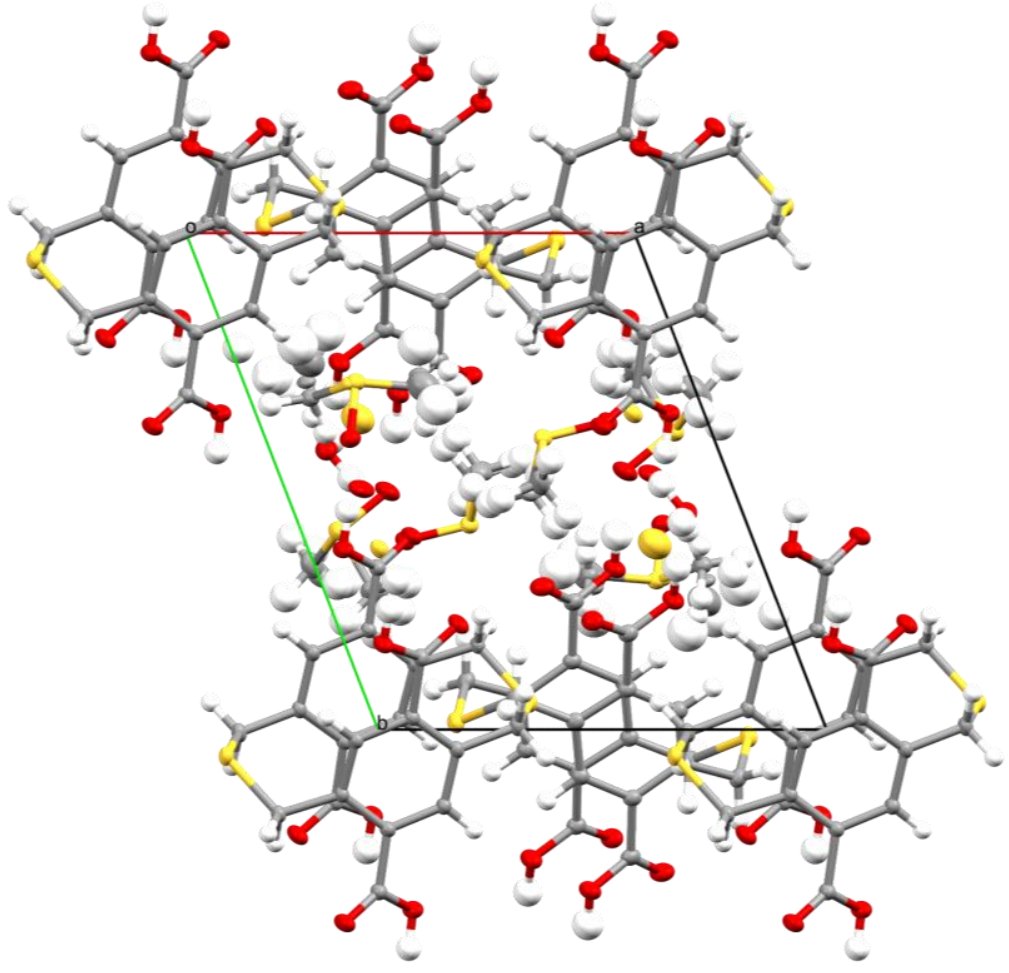

Figure S35. View of the unit cell of $\mathbf{3 b}$ viewed along the $c$-axis. 


\section{Computational Details:}

The geometries of all 2,11-dithia[3.3]paracyclophanes, monomers, and dimers were optimized at the $\omega \mathrm{B} 97 \mathrm{X}-\mathrm{D} / 6-31 \mathrm{G}$ level of theory as implemented in Gaussian $09 .{ }^{10}$ Frequency calculations were performed at the same computational level for all compounds, and no imaginary frequencies were found except in the case of transition states. Energies are rounded to the nearest $0.1 \mathrm{kcal} \mathrm{mol}^{-1}$. Relative energies given as difference between the noted compound and the most stable conformation. Strain enthalpy calculations were performed according to the methods outlined by Bachrach. ${ }^{11}$ Mismatch penalties calculated as difference between homochiral dimer energy and heterochiral dimer energy. Heterochiral dimer starting geometries obtained from the heterochiral dimer in the X-ray structure of $( \pm)$-1b. Optimized geometries rendered using CYLview20. ${ }^{12}$ NBO plots were rendered with Chemcraft 1.8. ${ }^{13}$

Computational Tables

Table S8. Energies of the low energy conformers of $\mathbf{d t}[3.3] \mathbf{p C p s}$ at different oxidation levels.

\begin{tabular}{|c|c|c|c|}
\hline Compound & a.u. & kcal mol $^{-1}$ & $\mathrm{E}_{\text {rel }} \mathrm{kcal} \mathrm{mol}^{-1}$ \\
\hline $\operatorname{dt}[3.3] p C p-S$ chair & -1415.300699 & -888114.6 & 0 \\
\hline $\mathrm{dt}[3.3] \mathrm{pCp}-S$ boat & -1415.300517 & -888114.5 & 0.1 \\
\hline $\operatorname{dt}[3.3] \mathrm{pCp}-\mathrm{SO}_{2}$ chair & -1715.643123 & -1076582.4 & 0 \\
\hline $\mathrm{dt}[3.3] \mathrm{pCp}-\mathrm{SO}_{2}$ boat & -1715.641743 & -1076581.5 & 0.9 \\
\hline $\mathbf{d t}[3.3] \mathrm{pCp}-S O$ chair trans in & -1565.469134 & -982346.8 & 0 \\
\hline dt[3.3]pCp-SO chair trans out & -1565.460934 & -982341.6 & 5.2 \\
\hline
\end{tabular}

Table S9. Energies of $\mathbf{d t}[3.3] \mathrm{pCp}-\mathrm{S}, \mathrm{dt}[3.3] \mathbf{p C p}-\mathrm{SO}_{2}$ (chair conformers) and their chair/boat transition states.

\begin{tabular}{|c|c|c|c|}
\hline Compound & a.u. & $\mathrm{kcal} \mathrm{mol}^{-1}$ & $\Delta G^{\ddagger}\left(\mathrm{kcal} \mathrm{mol}^{-1}\right)$ \\
\hline $\operatorname{dt}[3.3] \mathrm{pCp}-S$ & -1415.300699 & -888114.6 & - \\
\hline $\mathrm{dt}[3.3] \mathrm{pCp}-\mathrm{SO}_{2}$ & -1715.643123 & -1076582.4 & - \\
\hline $\operatorname{dt}[3.3] p C p-S$ TS & -1415.28696 & -888106.0 & 8.6 \\
\hline $\mathrm{dt}[3.3] \mathrm{pCp}-\mathrm{SO}_{2} \mathrm{TS}$ & -1715.629244 & -1076573.7 & 8.7 \\
\hline
\end{tabular}

Table S10. Energies of dt[3.3]pCpTA-S $\left(S_{\mathrm{p}}\right)$-1 conformers varying in amide and bridge conformation.

\begin{tabular}{ccccc}
\hline Conformer & a.u. & D (Debye) & ${\text { kcal } \mathbf{~ m o l}^{\mathbf{1}}}$ & $\mathbf{E}_{\mathbf{r e l}} \mathbf{~ k c a l ~} \mathbf{~ m o l}^{\mathbf{- 1}}$ \\
\hline boat anti- & -2246.86889 & 8.60 & -1409931.6 & 0.4 \\
chair anti- & -2246.86952 & 9.31 & -1409932.0 & 0.0 \\
chair syn- & -2246.86918 & 10.90 & -1409931.8 & 0.2 \\
boat syn- & -2246.86835 & 12.01 & -1409931.2 & 0.8 \\
\hline
\end{tabular}

Table S11. Energies of dt[3.3]pCpTA-SO $\left(S_{\mathrm{p}}\right)$-2 conformers varying in amide and bridge conformation.

\begin{tabular}{ccccc}
\hline Conformer & a.u. & D (Debye) & kcal mol $^{\mathbf{1}}$ & $\mathbf{E}_{\text {rel }} \mathbf{~ k a l ~ m o l}^{\mathbf{1}}$ \\
\hline boat anti- & -2547.20200 & 11.47 & -1598393.5 & 1.8 \\
boat syn- & -2547.20070 & 13.65 & -1598392.6 & 2.6 \\
chair anti- & -2547.20402 & 8.79 & -1598394.7 & 0.6 \\
chair syn- & -2547.20491 & 10.38 & -1598395.3 & 0.0 \\
\hline
\end{tabular}


Table S12. Dimer energies for conformers of $\operatorname{dt}[3.3] p C p T A-S\left(S_{\mathrm{p}}\right)-\mathbf{1}$

\begin{tabular}{|c|c|c|c|c|}
\hline Conformer & a.u. & D (Debye) & $\mathrm{kcal} \mathrm{mol}^{-1}$ & $\mathrm{E}_{\mathrm{rel}} \mathrm{kcal} \mathrm{mol}^{-\mathbf{1}}$ \\
\hline chair anti- & -4493.80983 & 12.13 & -2819908.4 & 0.0 \\
\hline chair syn- & -4493.80054 & 27.75 & -2819902.5 & 5.9 \\
\hline
\end{tabular}

Table S13. Dimer energies for conformers of dt[3.3]pCpTA-SO $\left(S_{\mathrm{p}}\right)-2$.

\begin{tabular}{ccccc}
\hline Conformer & a.u. & D (Debye) & kcal mol $^{-\mathbf{1}}$ & E $_{\text {rel }} \mathbf{~ k c a l ~} \mathbf{~ m o l}^{\mathbf{- 1}}$ \\
\hline chair anti- & -5094.481137 & 11.36 & -3196835.3 & 0.0 \\
chair syn- & -5094.471796 & 26.82 & -3196829.5 & 5.8 \\
\hline
\end{tabular}

Table S14. Calculated energies of homochiral (from Table S10, S11) and heterochiral dimers of 1 and 2 and their mismatch penalties (MMPs).

\begin{tabular}{ccccc}
\hline Dimer Structure & a.u. & D (Debye) & kcal mol $^{\mathbf{- 1}}$ & MMP \\
\hline heterochiral 1 & -4493.804219 & 2.76 & -2819904.8 & 3.5 \\
homochiral 1 & -4493.809827 & 12.13 & -2819908.4 & - \\
heterochiral 2 & -5094.469165 & 7.41 & -3196827.8 & 7.5 \\
homochiral 2 & -5094.481137 & 11.36 & -3196835.3 & - \\
\hline
\end{tabular}

Table S15. Calculated energies of dt[3.3]pCpTA-SO with differences in the sulfoxide orientation.

\begin{tabular}{ccccc}
\hline Compound & E (a.u.) & D (Debye) & E (kcal/mol) & E $_{\text {rel }} \mathbf{k c a l ~ m o l}^{-\mathbf{1}}$ \\
\hline chair anti- away & -2397.0293214 & 9.63 & -1504158.7 & 2.3 \\
chair anti- towards & -2397.0330535 & 8.85 & -1504161.0 & 0.0 \\
\hline
\end{tabular}

Table S16. Calculated energies of $\mathrm{dt}[3.3] \mathrm{pCpTA}-S O$ dimers.

\begin{tabular}{ccccc}
\hline compound & E (a.u.) & D (Debye) & E (kcal/mol) & E $_{\text {rel }} \mathbf{k c a l ~ m o l}^{\mathbf{1}}$ \\
\hline $\begin{array}{c}\text { dt[3.3]pCpTA-SO chair anti- } \\
\begin{array}{c}\text { away dimer } \\
\text { dt[3.3]pCpTA-SO chair anti- } \\
\text { towards dimer }\end{array}\end{array}$ & -4794.1350817 & 11.00 & -3008365.3 & 1.4 \\
\hline
\end{tabular}


Geometry Optimizations

(a)

(b)
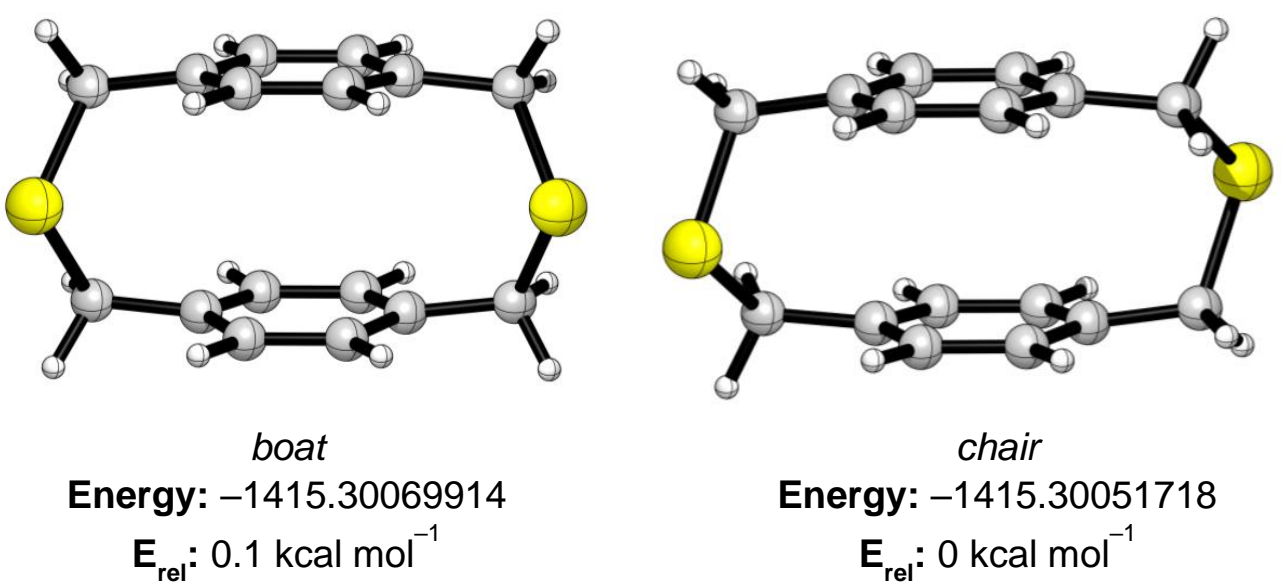

chair

Energy: -1415.30051718

$\mathrm{E}_{\mathrm{rel}}: 0 \mathrm{kcal} \mathrm{mol}^{-1}$

Figure S36. Optimized geometries and energies of boat (a) and chair (b) conformers of dt[3.3]pCp-S.

(a)

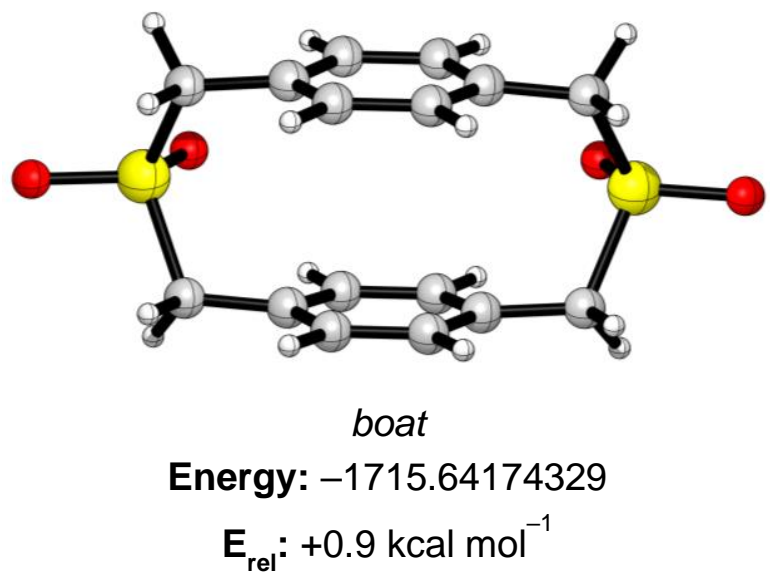

(b)

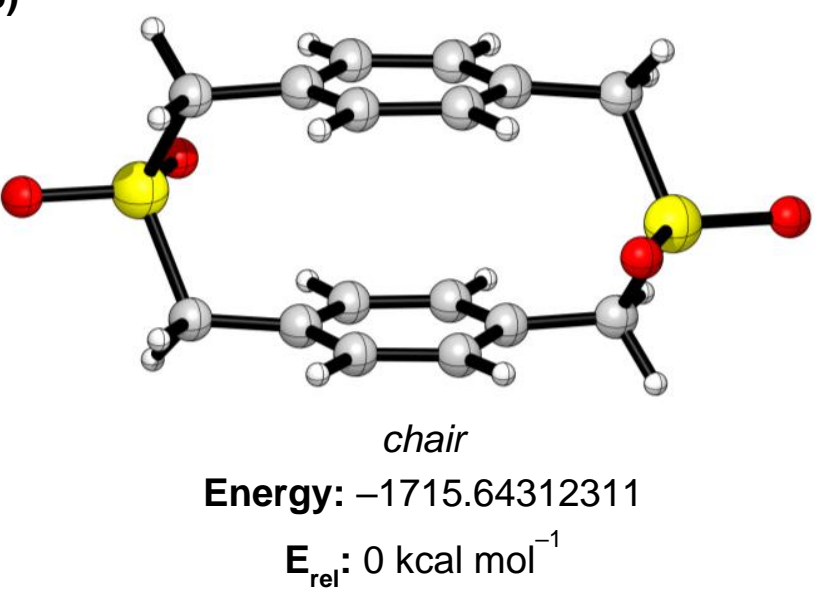

Figure S37. Optimized geometries and energies of boat (a) and chair (b) conformers of dt[3.3]pCp-SO .

(a)

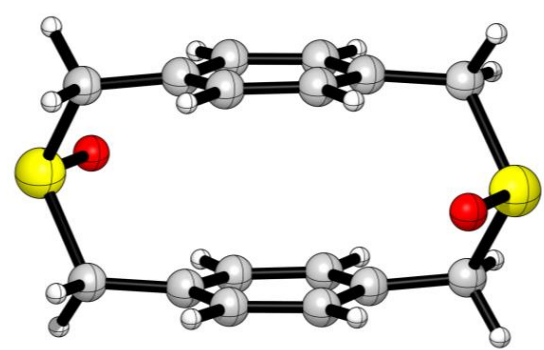

chair trans in

Energy: -1565.46913369

$\mathrm{E}_{\mathrm{rel}}: 0 \mathrm{kcal} \mathrm{mol}^{-1}$ (b)

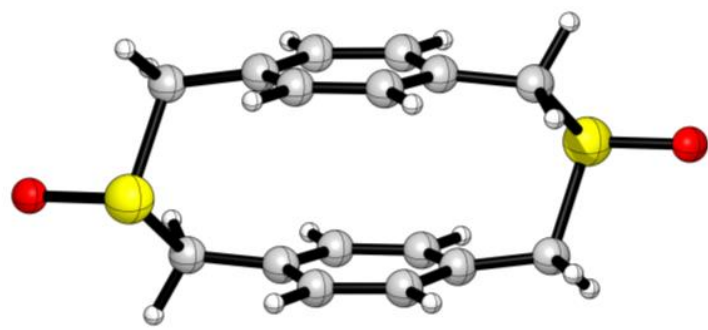

chair trans out

Energy: -1565.46093418

$\mathrm{E}_{\mathrm{rel}}: 5.2 \mathrm{kcal} \mathrm{mol}^{-1}$

Figure S38. Optimized geometries of chair trans in (a) and chair trans out (b) conformers of dt[3.3]pCp-SO. 
(a)

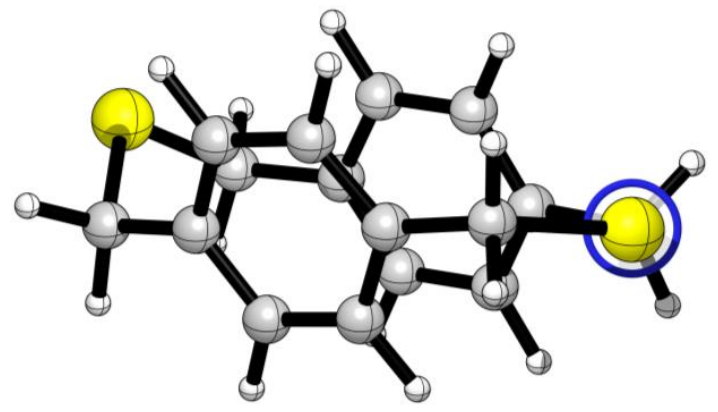

$\mathrm{dt}[3.3] \mathrm{pCp}-\mathrm{S}$ TS

Energy: -1415.30069914

$\mathrm{E}_{\mathrm{rel}}: 8.6 \mathrm{kcal} \mathrm{mol}^{-1}$ (b)

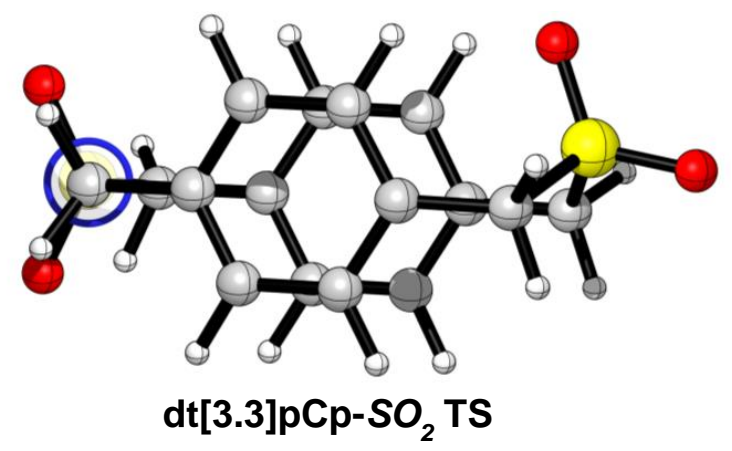

Energy: -1715.62924365

$\mathrm{E}_{\mathrm{rel}}: 8.7 \mathrm{kcal} \mathrm{mol}^{-1}$

Figure S39. Transition state geometries for the chair/boat interconversion process in $\mathbf{d t}[3.3] \mathbf{p C p}-\mathrm{S}$ (a) and $\mathbf{d t}[3.3] \mathbf{p C p}-\mathrm{SO}_{2}$ (b).

(a)

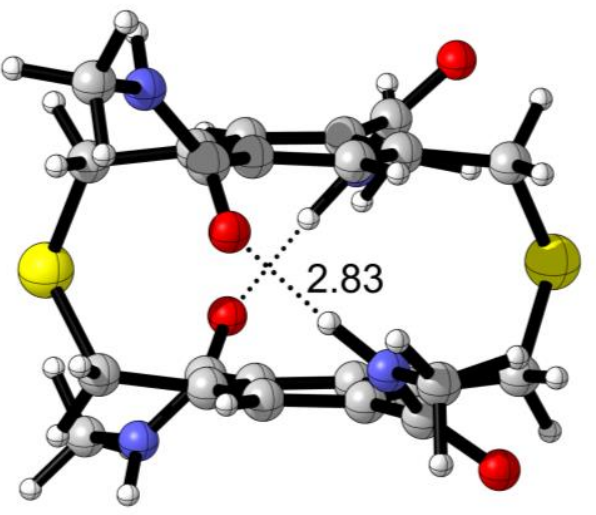

$\left(S_{\mathrm{p}}\right)-1$ boat anti-

Energy: -2246.86889133

E : $+0.4 \mathrm{kcal} \mathrm{mol}^{-1}$

(c)

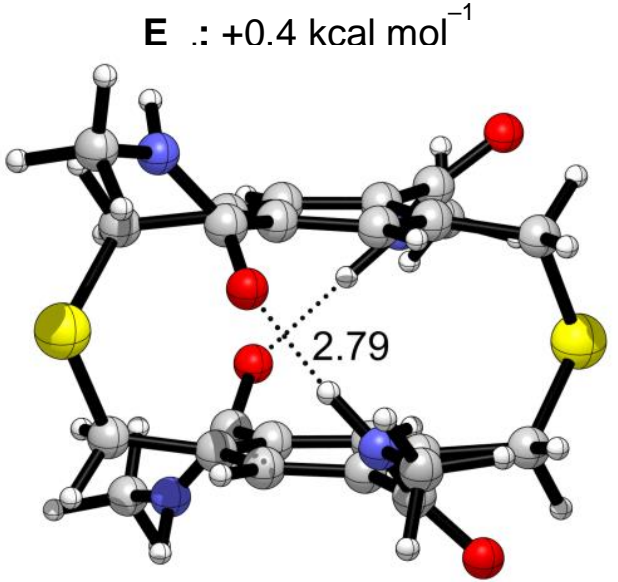

$\left(S_{p}\right)-1$ chair anti-

Energy: -2246.86952385

$$
\mathbf{E}_{\mathrm{rel}}: 0 \mathrm{kcal} \mathrm{mol}^{-1}
$$

(b)

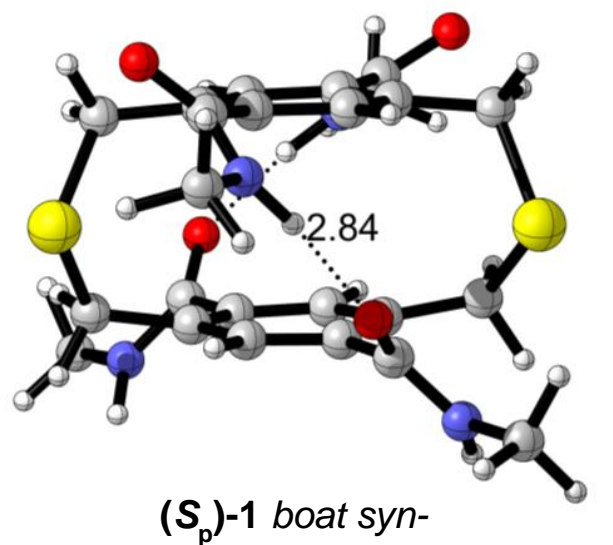

Energy: -2246.86835434

$\mathbf{E}_{\text {rel }}:+0.7 \mathrm{kcal} \mathrm{mol}^{-1}$

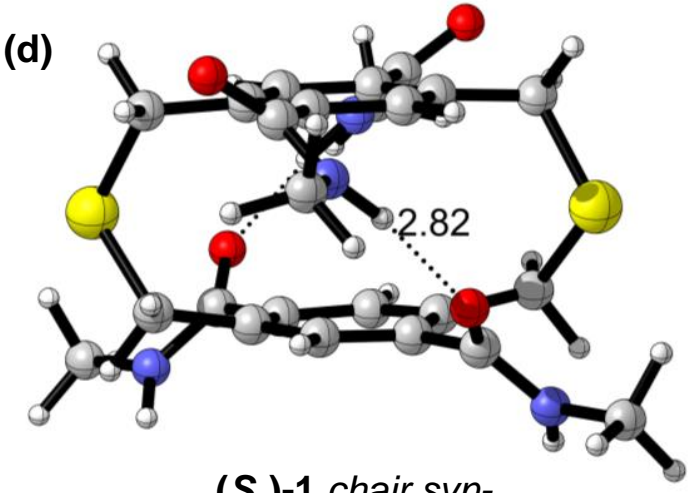

$\left(S_{p}\right)-1$ chair syn-

Energy: -2246.86917972

$\mathbf{E}_{\text {rel }}:+0.2 \mathrm{kcal} \mathrm{mol}^{-1}$

Figure S40. Optimized geometries of the four bridge and amide conformers of $\left(\boldsymbol{S}_{\mathrm{p}}\right)$-1. 


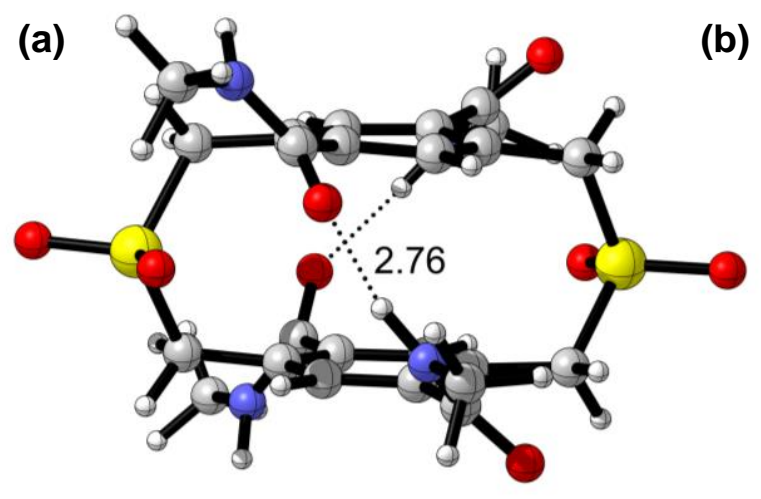

$\left(S_{p}\right)-2$ chair anti-

Energy: -2547.20401869

$\mathbf{E}_{\mathrm{rel}}:+0.6 \mathrm{kcal} \mathrm{mol}^{-1}$

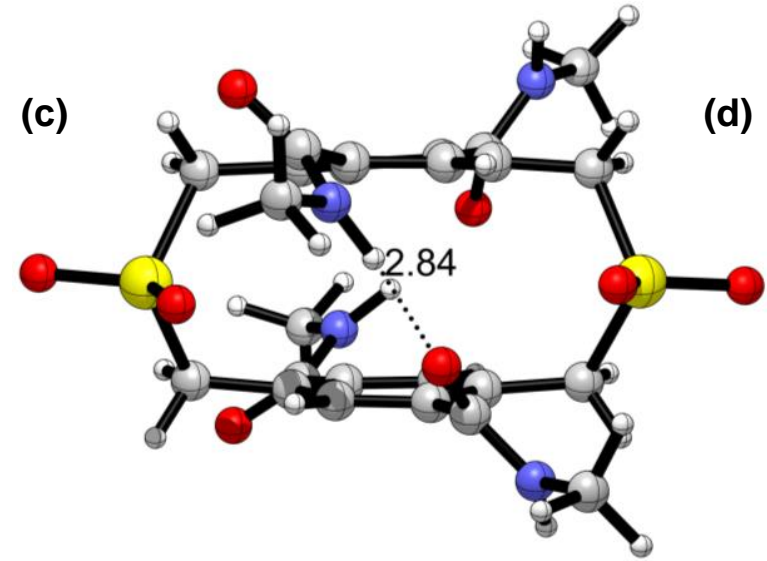

$\left(S_{\mathrm{p}}\right)-2$ boat anti-

Energy: -2547.20199977

$\mathrm{E}_{\mathrm{rel}}:+1.8 \mathrm{kcal} \mathrm{mol}^{-1}$

(b)

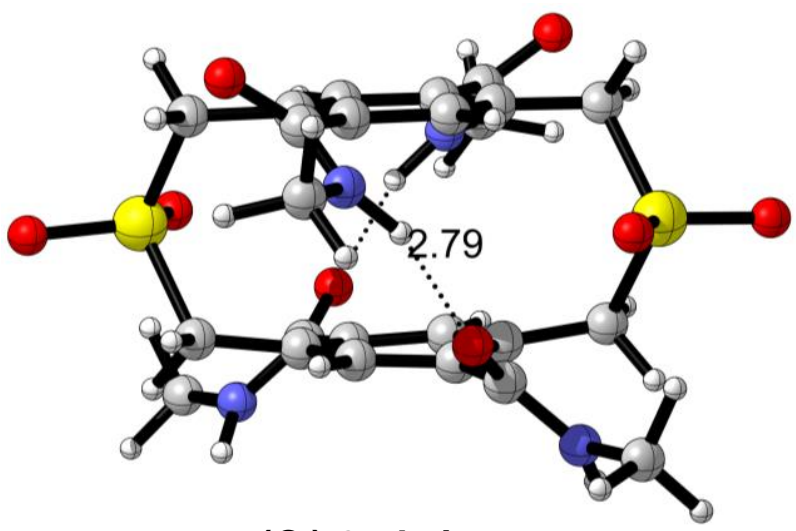

$\left(S_{\mathrm{p}}\right)-2$ chair syn-

Energy: -2547.20491395

$\mathrm{E}_{\mathrm{rel}}: 0 \mathrm{kcal} \mathrm{mol}^{-1}$

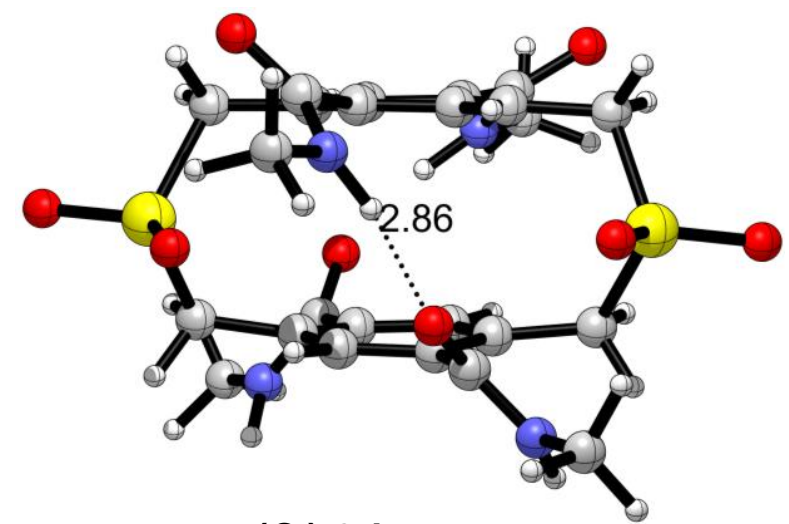

$\left(S_{\mathrm{p}}\right)-2$ boat syn-

Energy: -2547.20069965

$\mathrm{E}_{\mathrm{rel}}:+2.7 \mathrm{kcal} \mathrm{mol}^{-1}$

Figure S41. Optimized geometries of the four bridge and amide conformers of $\left(\boldsymbol{S}_{\mathrm{p}}\right)$-2 . 


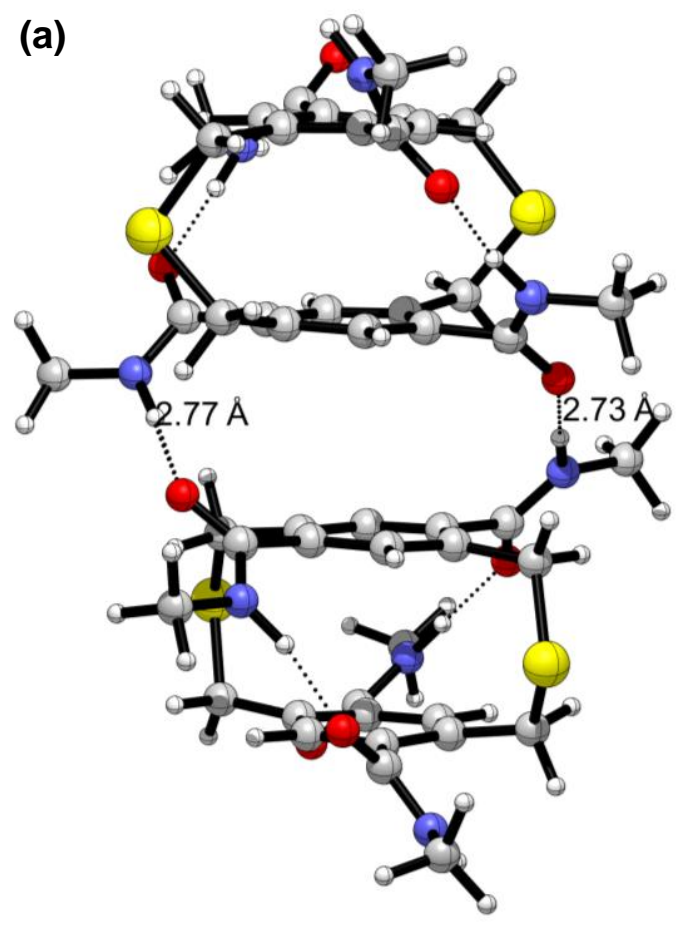

$\left(S_{p}\right)-1$ chair anti- dimer

Energy: -4493.80982677

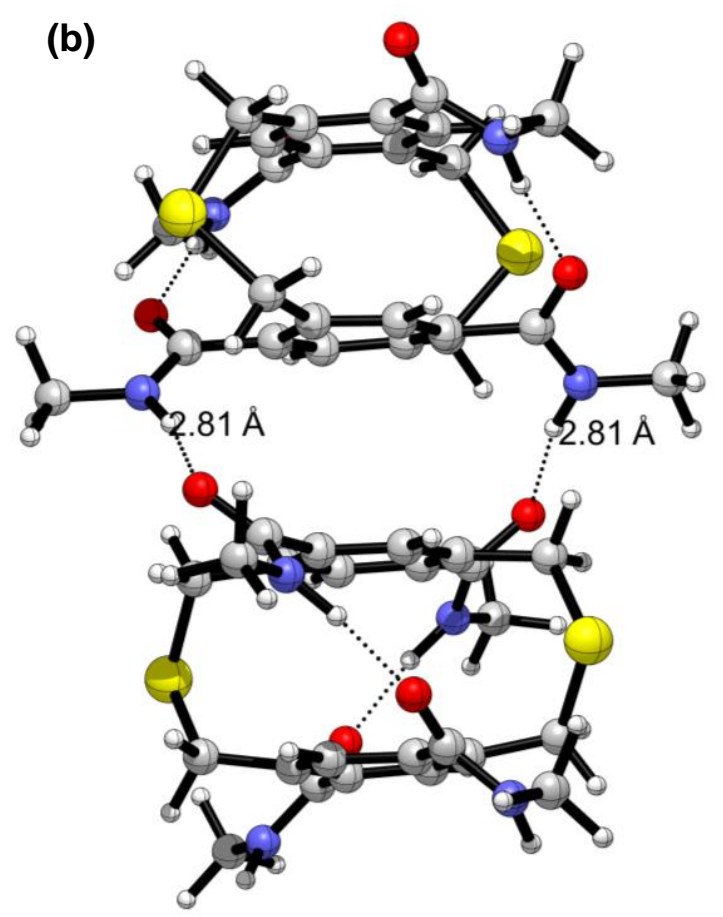

$\left(S_{\mathrm{p}}\right)-1$ chair syn- dimer Energy: -4493.80054211 $\mathbf{E}_{\mathrm{rel}}:+5.9 \mathrm{kcal} \mathrm{mol}^{-1}$

Figure S42. Optimized dimers of $\left(\boldsymbol{S}_{\mathbf{p}}\right)$-1 in the chair anti- conformation (a) and the chair syn- conformation (b). 


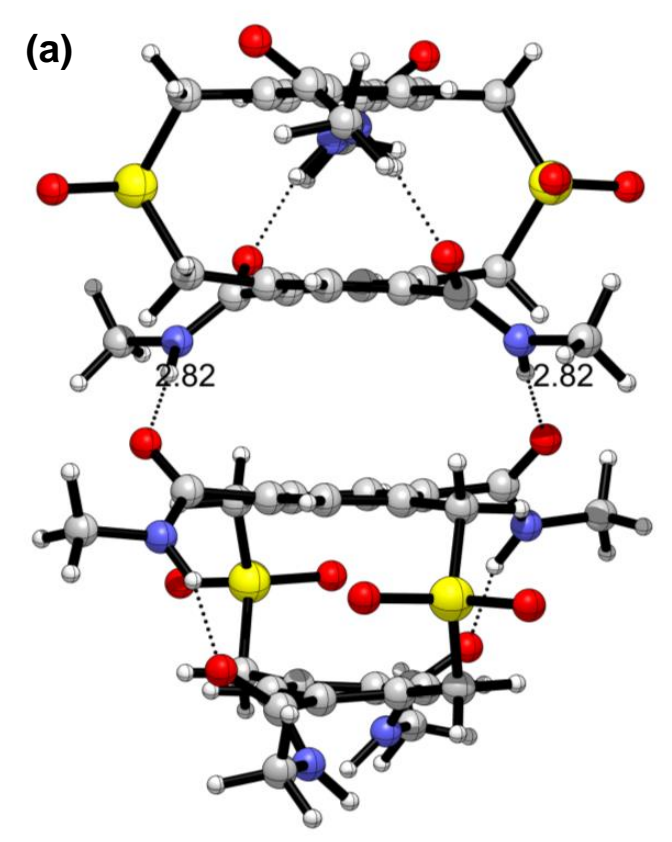

$\left(S_{p}\right)-2$ chair syn- dimer

Energy: -5094.48113661

$\mathrm{E}_{\mathrm{rel}}:+5.8 \mathrm{kcal} \mathrm{mol}^{-1}$ (b)

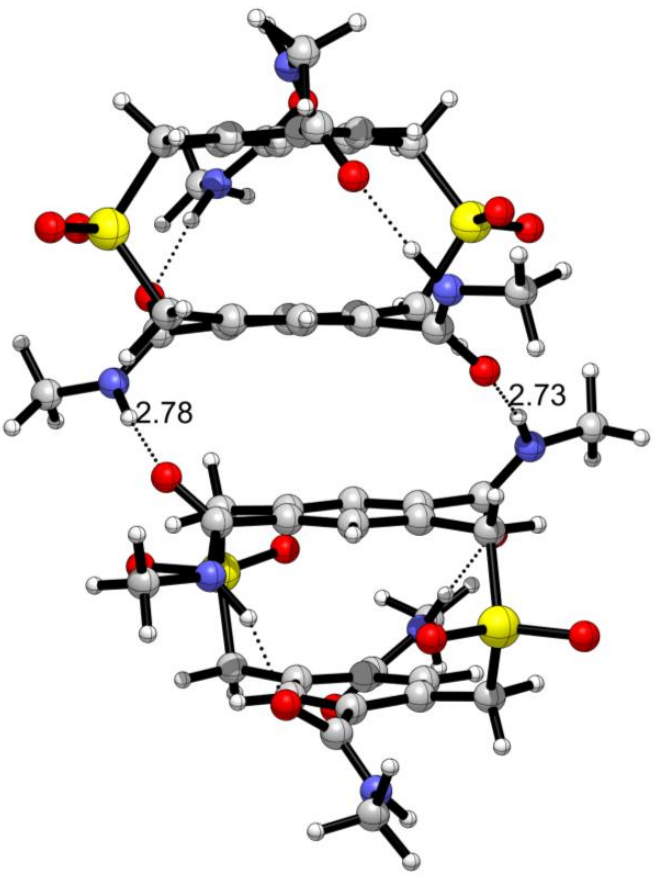

$\left(S_{p}\right)-2$ chair anti- dimer

Energy: -5094.48113661

$\mathrm{E}_{\mathrm{rel}}: 0 \mathrm{kcal} \mathrm{mol}^{-1}$

Figure S43. Optimized geometries of $\left(\boldsymbol{S}_{\mathbf{p}}\right)$-2 dimers in the chair syn- conformation (a) and chair anti- conformation (b). Distances in $\AA$.

(a)

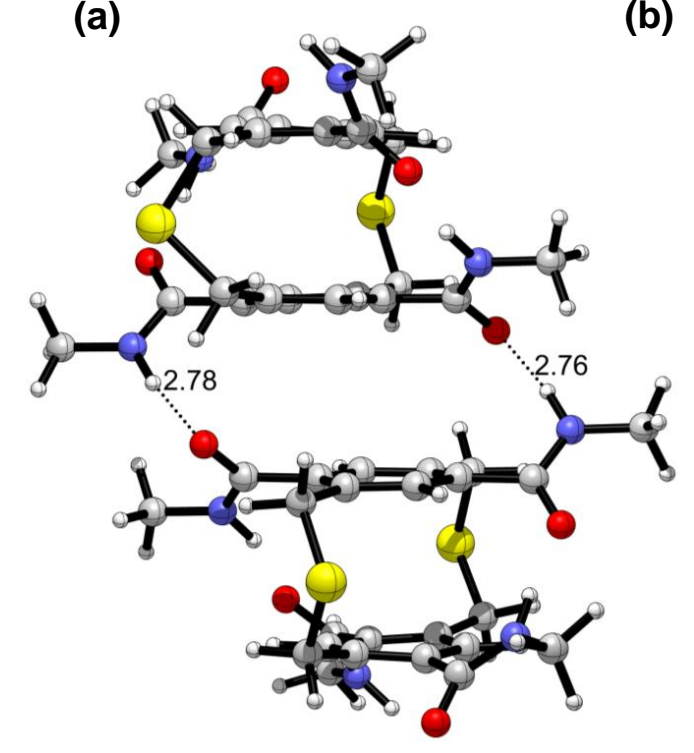

heterochiral 1 anti- dimer

Energy: -4493.804219 a.u.

MMP: $3.5 \mathrm{kcal} \mathrm{mol}^{-1}$

(b)

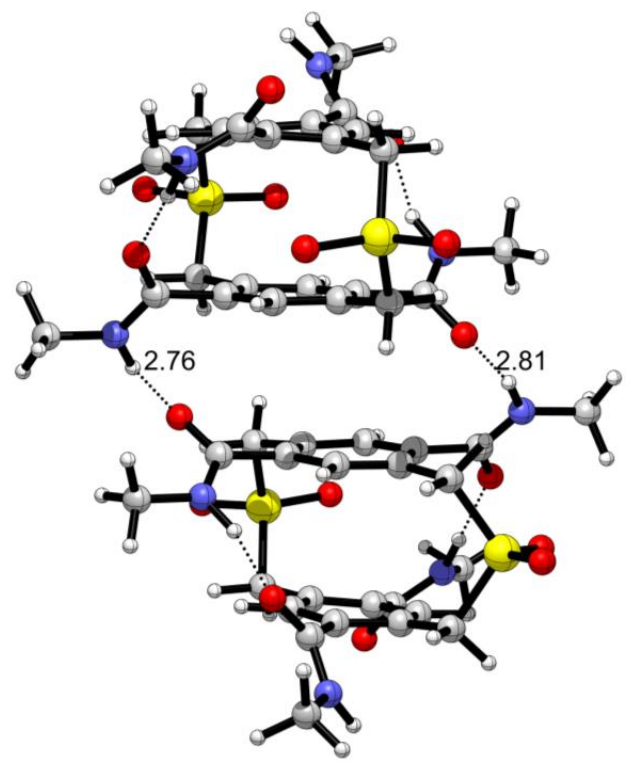

heterochiral 2 anti- dimer

Energy: -5094.469165 a.u.

MMP: $7.5 \mathrm{kcal} \mathrm{mol}^{-1}$

Figure S44. Optimized heterochiral dimers of 1 (a) and 2 (b) and their mismatch penalties. Distances in $\AA$. 


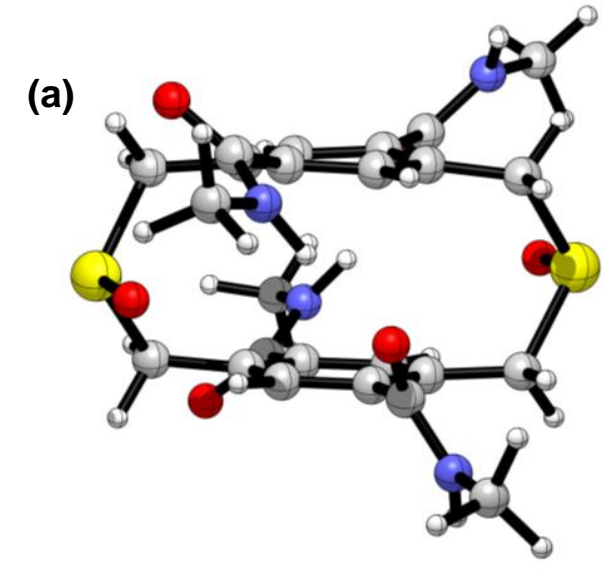

chair anti-

S=O towards

Energy: -2397.02932144

$\mathbf{E}_{\mathrm{rel}}: 0 \mathrm{kcal} \mathrm{mol}^{-1}$

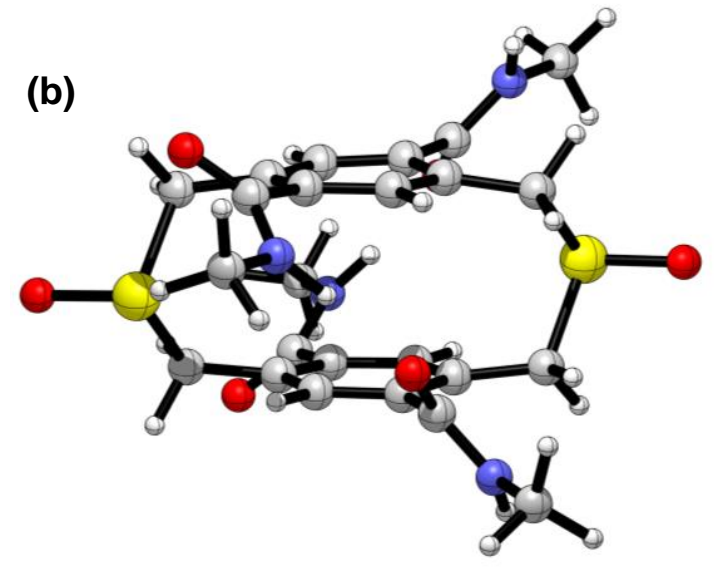

chair anti-

$S=0$ away

Energy: -2397.02932144

$\mathbf{E}_{\text {rel }}:+2.3 \mathrm{kcal} \mathrm{mol}^{-1}$

Figure S45. Optimized geometries of $\mathbf{d t}[3.3] \mathbf{p C p}-\mathbf{S O}$ in the chair anti-conformation with $\mathrm{S}=\mathrm{O}$ towards (a) and away (b).
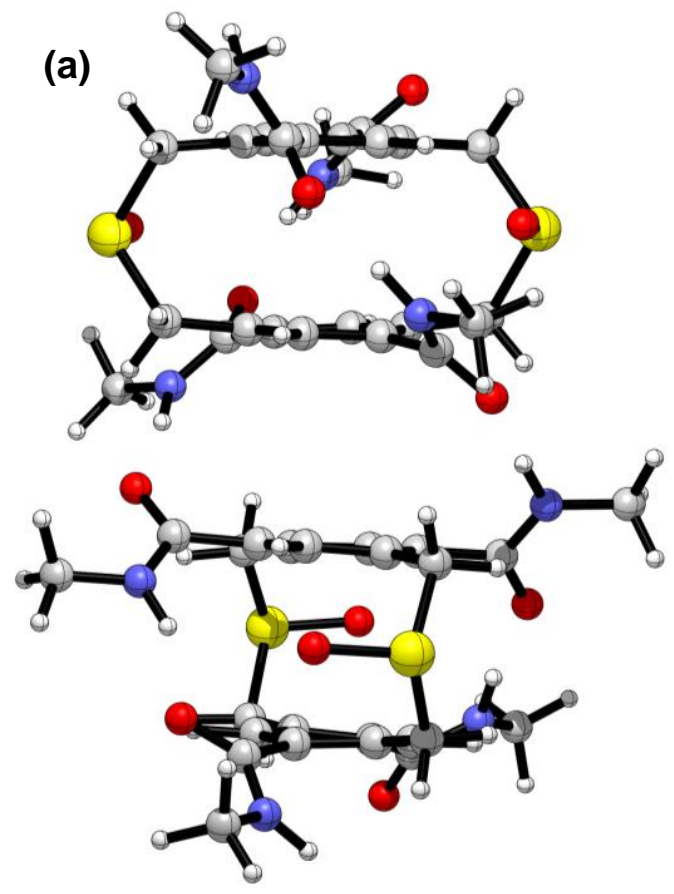

(b)

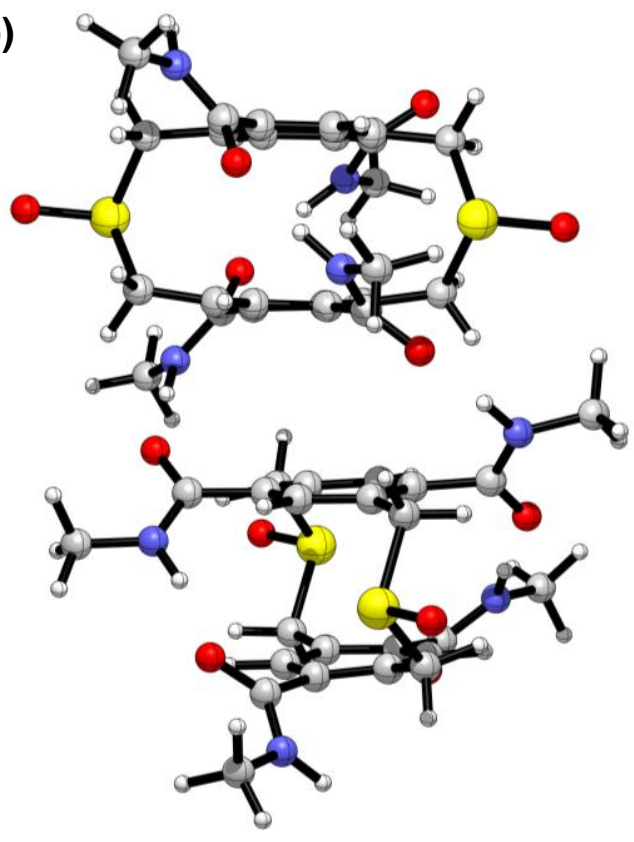

dt[3.3]pCpTA-SO chair anti- dimer

$S=0$ towards

Energy: -4794.137244

$\mathbf{E}_{\mathrm{rel}}: 0 \mathrm{kcal} \mathrm{mol}^{-1}$

dt[3.3]pCpTA-SO chair anti- dimer

$$
\text { S=O away }
$$

Energy: -4794.13508166

$\mathbf{E}_{\text {rel }}:+1.4 \mathrm{kcal} \mathrm{mol}^{-1}$

Figure S46. Optimized geometries of dt[3.3]pCpTA-SO dimers with $\mathrm{S}=\mathrm{O}$ towards (a) and away (b). 
Conformational Analysis

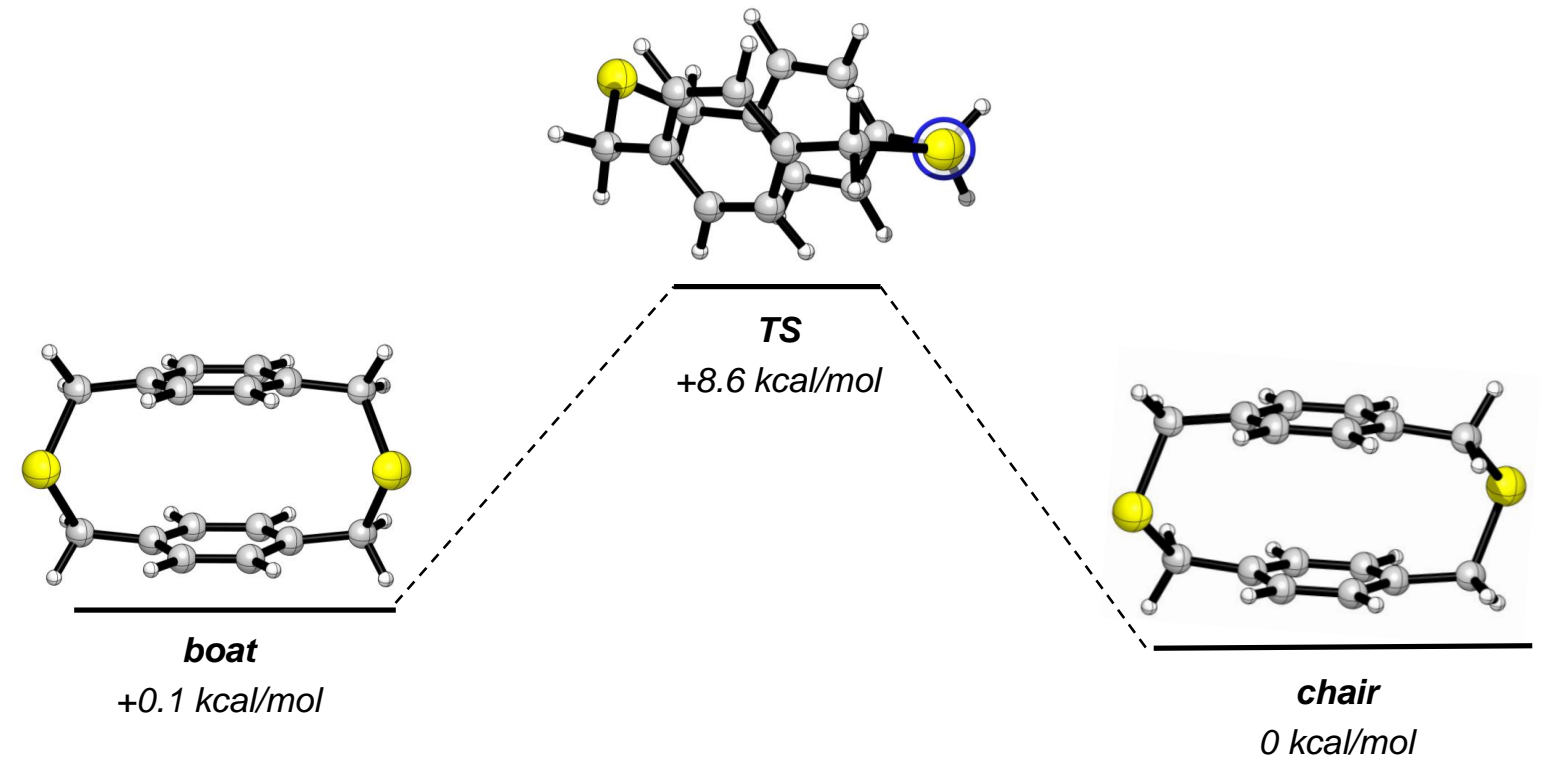

Figure S47. Relative energies for the chair/boat interconversion of $\mathbf{d t}[3.3] \mathbf{p C p}-\mathbf{S}$. Energies not drawn to scale.

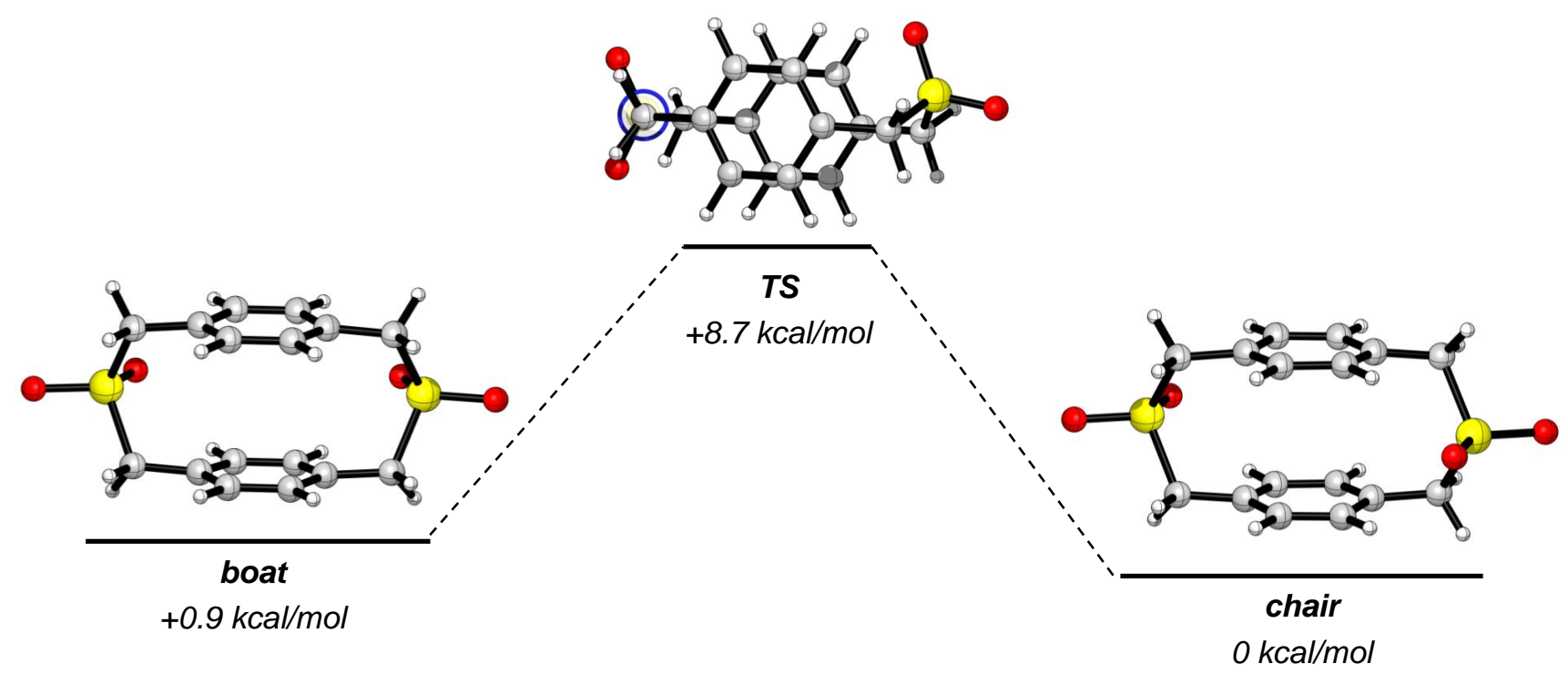

Figure S48. Relative energies for the chair/boat interconversion of dt[3.3]pCp-SO 2 . Energies not drawn to scale. 
Strain Enthalpy Calculations

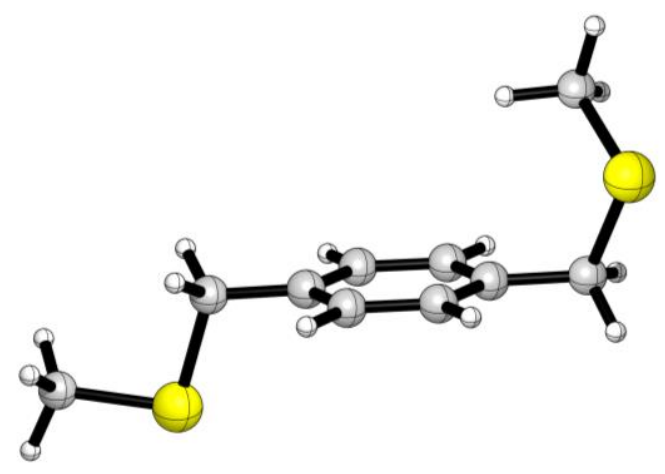

1,4-bis((methylthio)methyl)benzene

Energy: -1185.398244

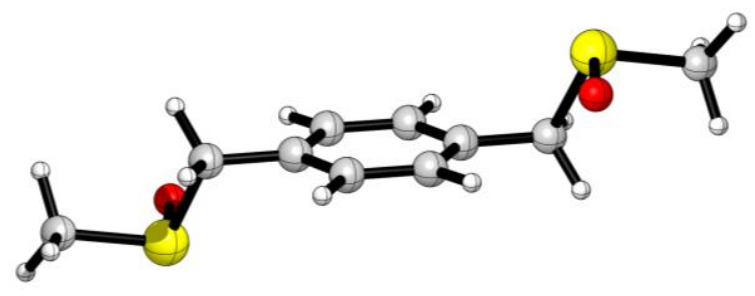

1,4-meso-bis((methylsulfinyl)methyl)benzene Energy: -1335.554156

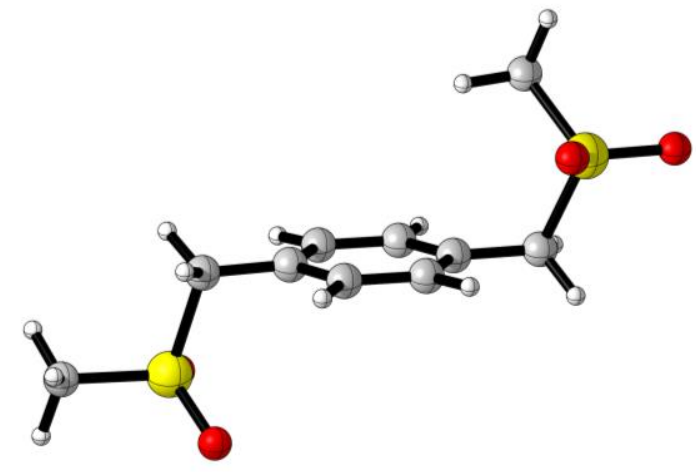

1,4-bis((methylsulfonyl)methyl)benzene Energy: -1485.718602

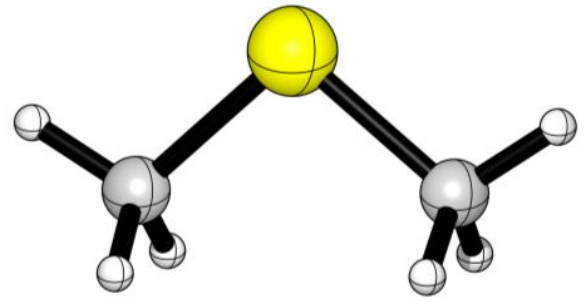

dimethylsulfide (DMS)

Energy: -477.870972

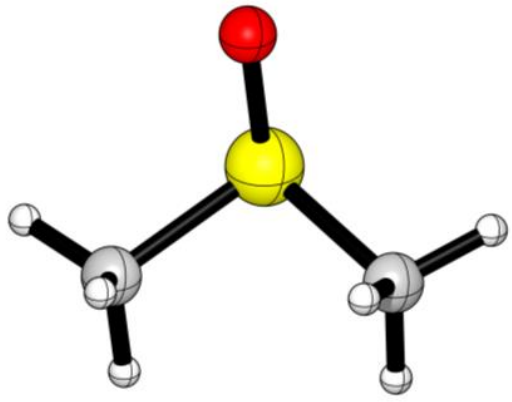

dimethylsulfoxide (DMSO)

Energy: -552.946879

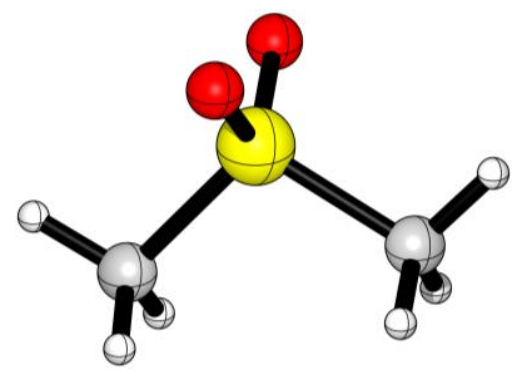

dimethylsulfone ( $\mathrm{DMSO}_{2}$ )

Energy: -628.027439

Figure S49. Optimized structures and energies (a.u.) of small molecules used to calculate strain energy of dt[3.3]pCps. 

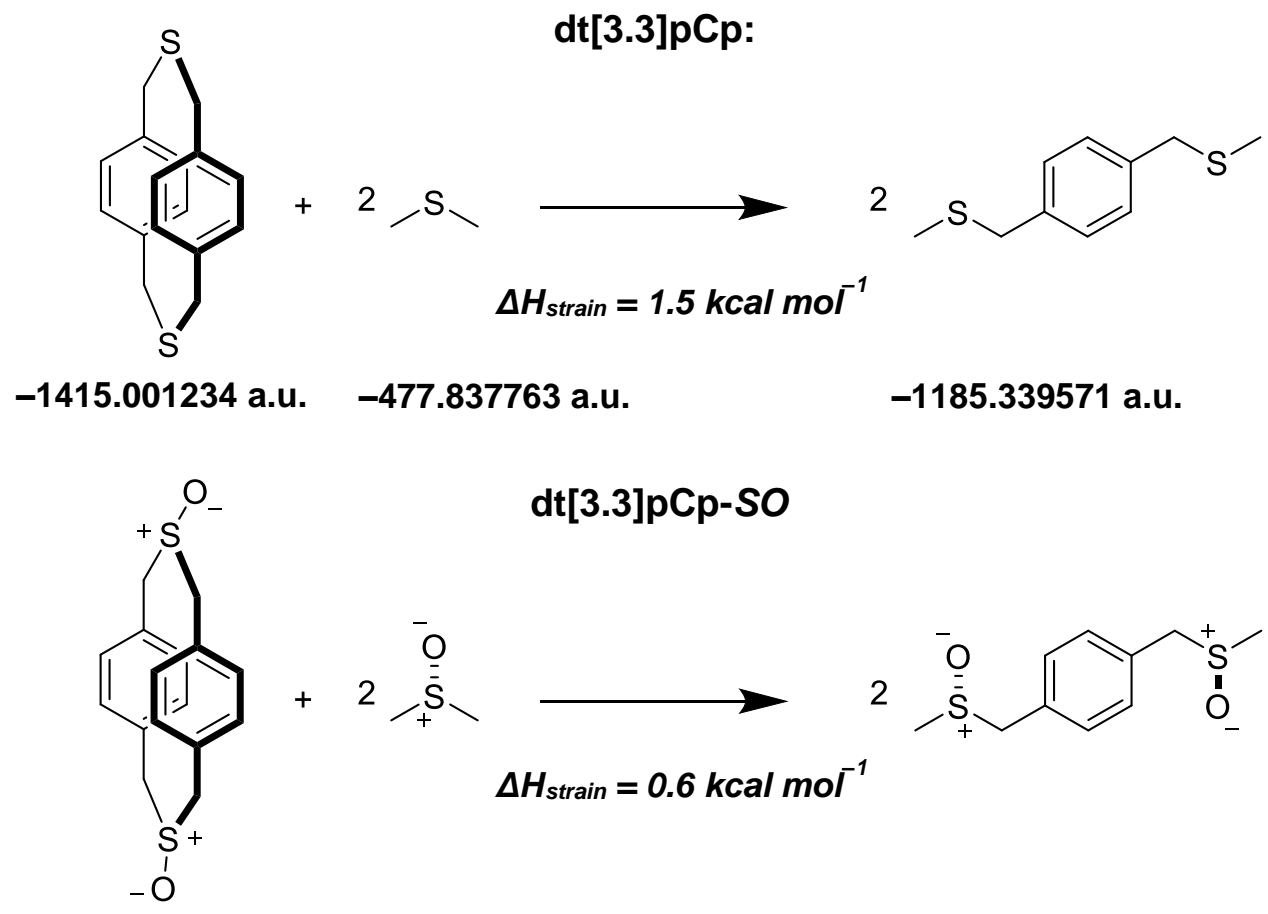

-1565.161569 a.u. $\quad-552.910749$ a.u. $\quad-1335.49198$ a.u.

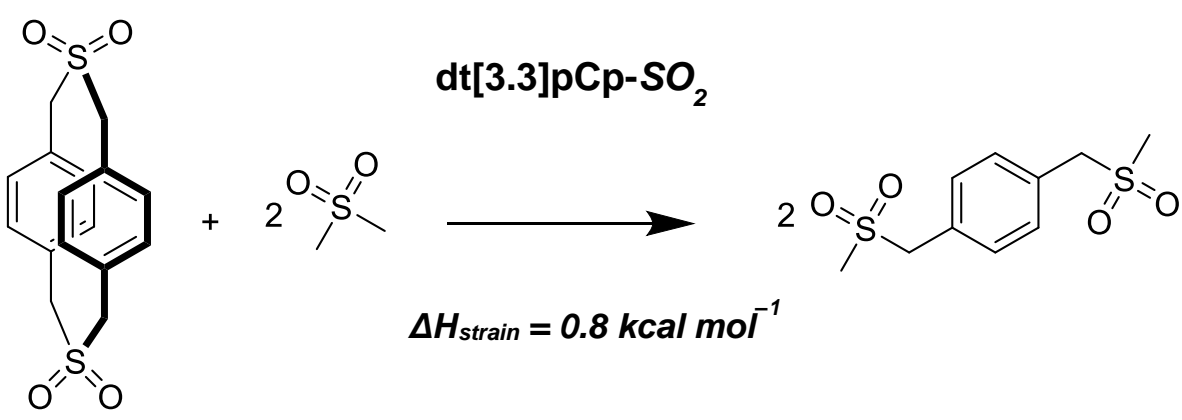

-1715.326761 a.u. $\quad-627.989326$ a.u. $\quad-1485.652097$ a.u.

Figure S50. Homodesmotic reactions designed to calculate the strain enthalpy of $\mathbf{d t}[\mathbf{3 . 3}] \mathbf{p C p s}$ (most stable conformer of dt[3.3]pCps from Table S8 was chosen for calculations). 


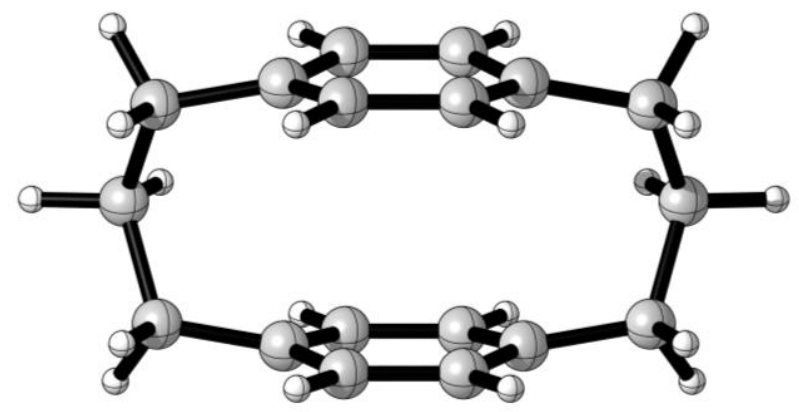

[3.3]pCp boat

Energy: -697.302538 a.u.

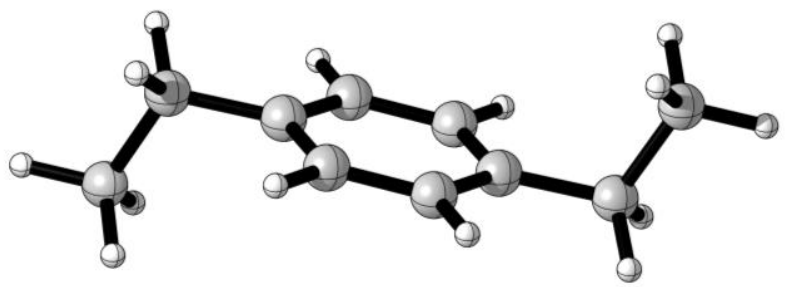

1,4-diethylbenzene

Energy: $-\mathbf{3 8 9 . 1 1 7 0 3 4}$ a.u.

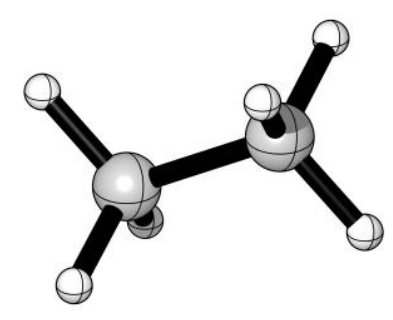

ethane

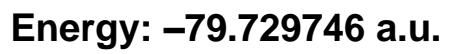

Figure S51. Energies of geometry-optimized molecules used for homodesmotic [3.3]pCp strain enthalpy calculations.<smiles></smiles>

$+4 \mathrm{C}_{2} \mathrm{H}_{6}$<smiles>[R]C(C)C</smiles>

2<smiles>CCc1ccc(CC)cc1</smiles>

$\Delta H=6.1 \mathrm{kcal} \mathrm{mol}^{-1}$

-697.247511 a.u. $\quad-79.729746$ a.u.

-389.069329 a.u. $\quad-118.967124$ a.u.

Figure S52. Homodesmotic reactions designed to calculate the strain enthalpy of [3.3]pCp (boat conformer of [3.3]pCp). ${ }^{11}$

Table S17. Table of strain enthalpy calculations from Figure S50.

\begin{tabular}{cccc}
\hline Compound & reactants & products & strain enthalpy \\
\hline $\mathbf{d t}[3.3] \mathbf{p C p}-\boldsymbol{S}$ & -1487622.19 & -1487623.68 & 1.5 \\
$\mathbf{d t}[3.3] \mathbf{C p}-\mathrm{SO}$ & -1676067.25 & -1676067.81 & 0.6 \\
$\mathbf{d t}[3.3] \mathbf{C C p}-\mathrm{SO}_{2}$ & -1864522.37 & -1864521.61 & 0.8 \\
\hline
\end{tabular}




\section{Natural Bond Orbital Plots}
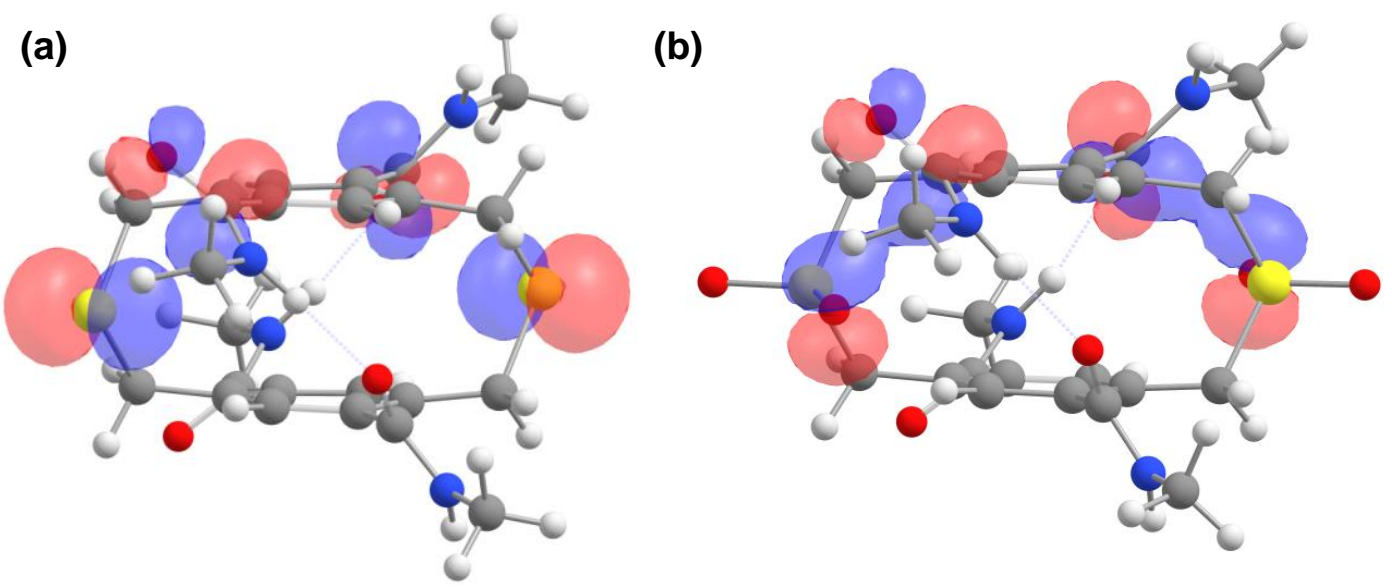

Figure S53. NBO plots showing sum of bridge non-bonding orbitals and amide $\pi^{*}$ orbitals for $\left(\boldsymbol{S}_{\mathrm{p}}\right)-\mathbf{1}(\mathrm{a})$ and $\left(\boldsymbol{S}_{\mathrm{p}}\right)-\mathbf{2}$ (b). The nonbonding orbitals of bridge sulfur is shown in (a) where the non-bonding orbitals of the sulfone oxygen are shown in (b).
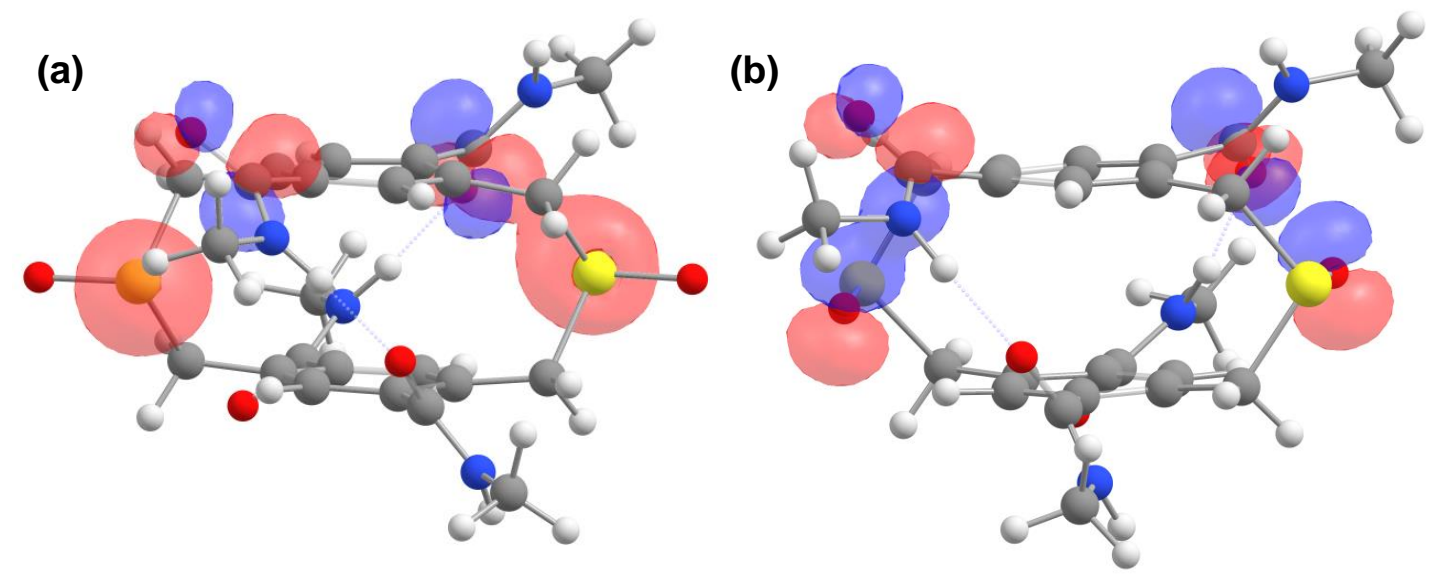

Figure S54. NBO plots showing sum of bridge non-bonding orbitals and amide $\pi^{*}$ orbitals for dt[3.3]pCpTA-SO away (a) and towards (b). Non-bonding orbitals on sulfur shown in (a) whereas non-bonding oribtals on sulfoxide oxygen shown in (b). 
Frontier Molecular Orbital Plots

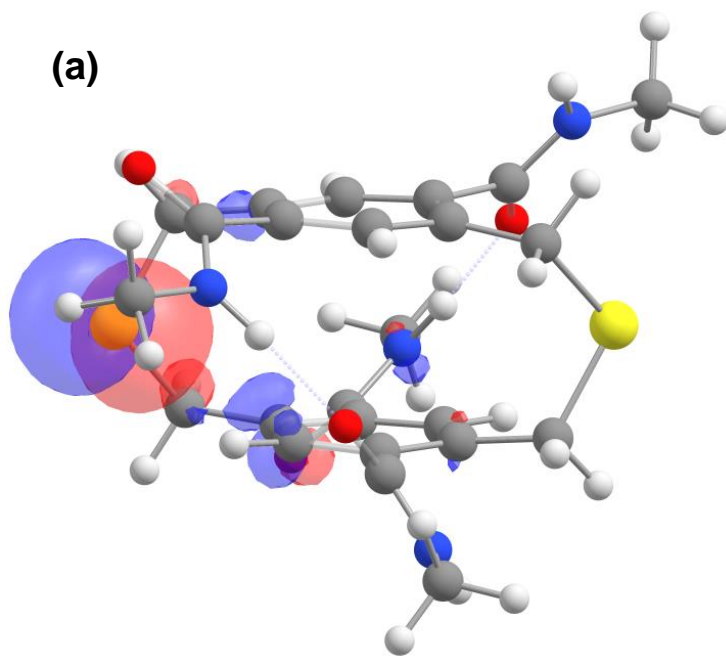

$\left(S_{p}\right)-1$ chair anti- HOMO (b)

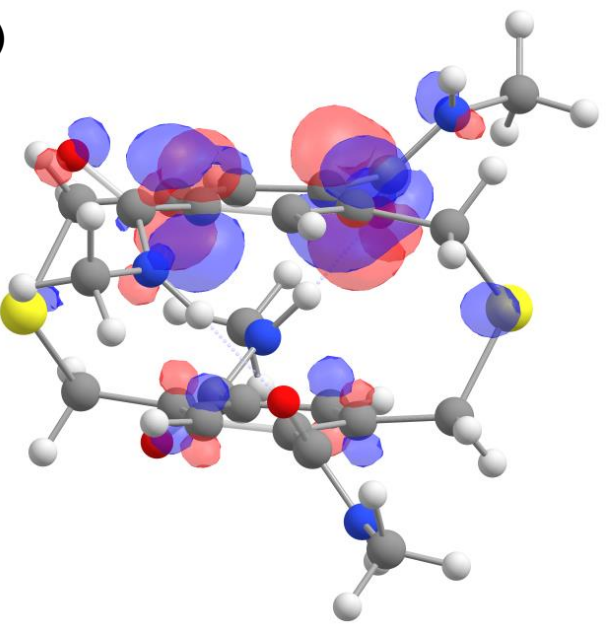

$\left(S_{p}\right)-1$ chair anti- LUMO

Figure S55. Molecular orbital plots of the HOMO (a) and LUMO (b) of $\left(\boldsymbol{S}_{\mathbf{p}}\right)$-1 chair anti- conformer.

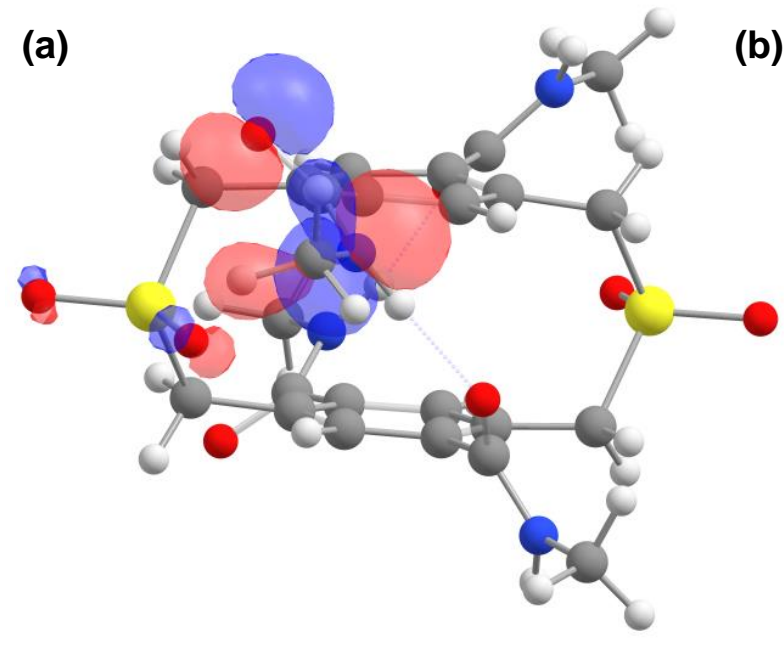

$\left(S_{p}\right)-2$ chair anti- HOMO

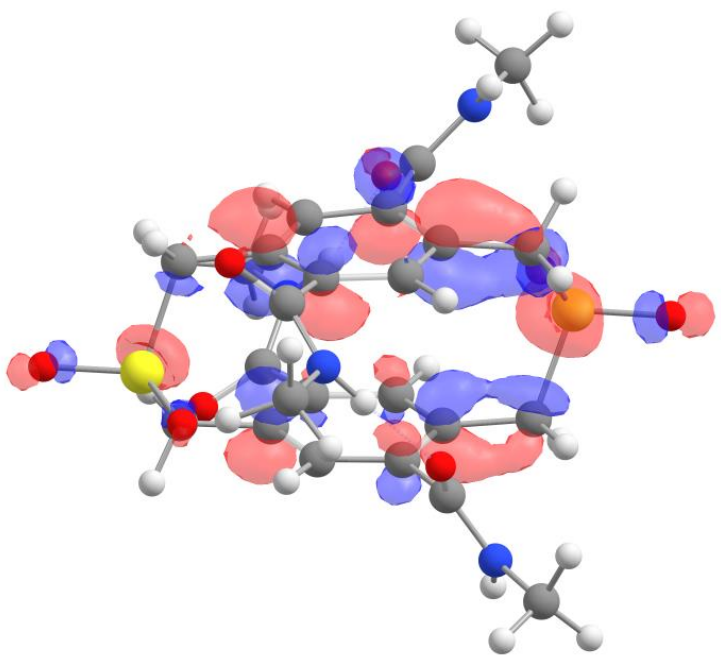

$\left(S_{p}\right)-2$ chair anti- LUMO

Figure 56. Molecular orbital plots of the HOMO (a) and LUMO (b) of $\left(\boldsymbol{S}_{\mathbf{p}}\right)-\mathbf{2}$ chair anti- conformer. 
Cartesian Coordinates of Optimized Structures

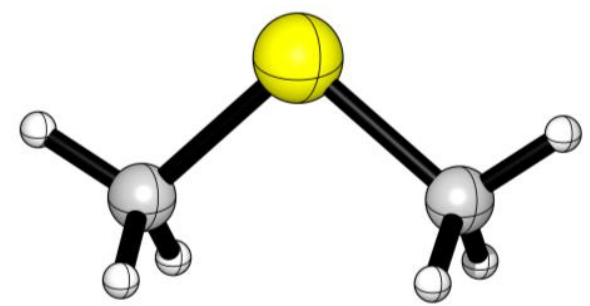

dimethylsulfide (DMS)

Energy: -477.870972

$\begin{array}{lrrr}\mathrm{S} & -0.0000180000 & 0.6850460000 & -0.0000120000 \\ \mathrm{C} & 1.4164040000 & -0.5352760000 & -0.0000390000 \\ \mathrm{C} & -1.4163620000 & -0.5352900000 & 0.0000100000 \\ \mathrm{H} & 1.3789270000 & -1.1583820000 & -0.8955180000 \\ \mathrm{H} & 1.3789770000 & -1.1577890000 & 0.8959210000 \\ \mathrm{H} & 2.3378760000 & 0.0475620000 & -0.0000930000 \\ \mathrm{H} & -2.3378260000 & 0.0475460000 & 0.0005160000 \\ \mathrm{H} & -1.3792980000 & -1.1578150000 & -0.8959370000 \\ \mathrm{H} & -1.3786220000 & -1.1584580000 & 0.8954800000\end{array}$

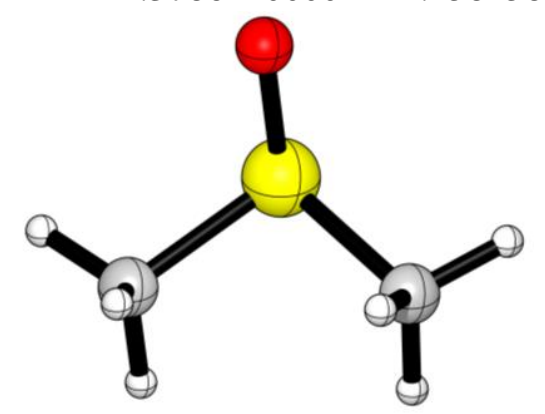

dimethylsulfoxide (DMSO)

Energy: -552.946879

$\begin{array}{lrrr}\mathrm{S} & 0.0000010000 & -0.1908130000 & -0.4945530000 \\ \mathrm{O} & 0.0000060000 & -1.6090040000 & 0.3970120000 \\ \mathrm{C} & 1.4056730000 & 0.8280860000 & 0.2203080000 \\ \mathrm{C} & -1.4056790000 & 0.8280760000 & 0.2203090000 \\ \mathrm{H} & 1.3619390000 & 1.8369630000 & -0.1919120000 \\ \mathrm{H} & 2.3301170000 & 0.3298580000 & -0.0677620000 \\ \mathrm{H} & 1.3027330000 & 0.8272320000 & 1.3061990000 \\ \mathrm{H} & -1.3027250000 & 0.8272520000 & 1.3061980000 \\ \mathrm{H} & -1.3619690000 & 1.8369450000 & -0.1919360000 \\ \mathrm{H} & -2.3301170000 & 0.3298240000 & -0.0677380000\end{array}$

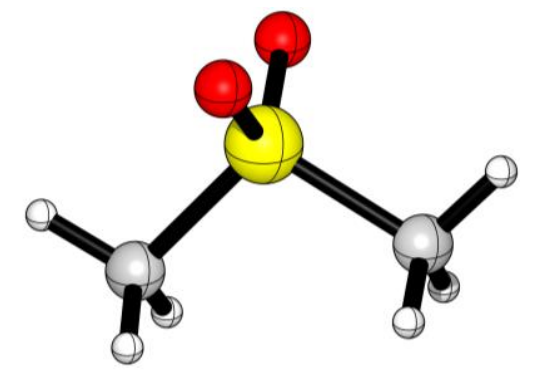




\begin{tabular}{lccc}
\multicolumn{4}{l}{ dimethylsulfone (DMSO} \\
Energy: -628.027439 \\
S & 0.0000000000 & -0.1747840000 & -0.0009640000 \\
$\mathrm{O}$ & 0.0000000000 & -0.9726820000 & -1.4249290000 \\
$\mathrm{O}$ & 0.0000000000 & -0.9933570000 & 1.4110730000 \\
$\mathrm{C}$ & 1.4694780000 & 0.9677690000 & 0.0066250000 \\
$\mathrm{C}$ & -1.4694780000 & 0.9677690000 & 0.0066260000 \\
$\mathrm{H}$ & 1.4244590000 & 1.5748090000 & -0.8959800000 \\
$\mathrm{H}$ & 2.3444850000 & 0.3198430000 & 0.0010600000 \\
$\mathrm{H}$ & 1.4259110000 & 1.5611600000 & 0.9183050000 \\
$\mathrm{H}$ & -1.4259100000 & 1.5611600000 & 0.9183050000 \\
$\mathrm{H}$ & -1.4244600000 & 1.5748090000 & -0.8959790000 \\
$\mathrm{H}$ & -2.3444850000 & 0.3198420000 & 0.0010620000
\end{tabular}

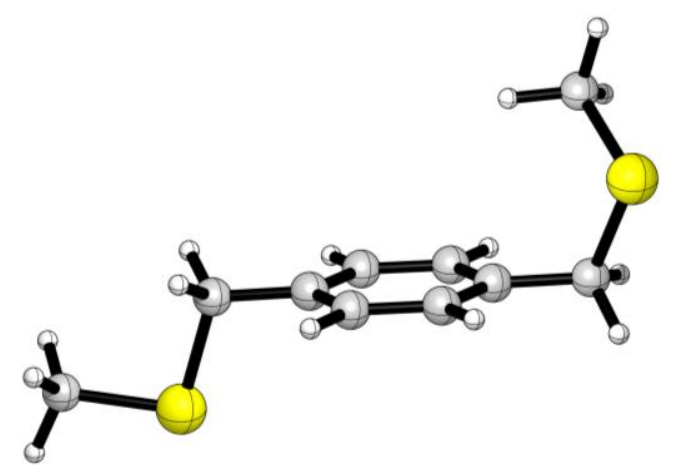

\begin{tabular}{lrrr}
\multicolumn{5}{l}{ 1,4-bis((methylthio)methyl)benzene } \\
Energy: -1185.398244 \\
S & 4.1780880000 & -0.3807400000 & 0.3120860000 \\
S & -3.7897910000 & -0.2859280000 & -0.5054360000 \\
C & 3.8209780000 & 1.3905220000 & 0.7875210000 \\
C & -5.3763830000 & 0.1982400000 & 0.3583880000 \\
C & -2.5658330000 & 0.4011870000 & 0.7654680000 \\
C & 2.8827690000 & -0.5831780000 & -1.0522950000 \\
$\mathrm{C}$ & 1.4760130000 & -0.3346880000 & -0.5868490000 \\
$\mathrm{C}$ & 0.8260050000 & -1.2466390000 & 0.2556730000 \\
$\mathrm{C}$ & -0.4734260000 & -1.0085230000 & 0.6918740000 \\
$\mathrm{C}$ & -1.1574470000 & 0.1528440000 & 0.3069670000 \\
$\mathrm{C}$ & -0.5095210000 & 1.0642450000 & -0.5329720000 \\
$\mathrm{C}$ & 0.7918590000 & 0.8238570000 & -0.9723860000 \\
$\mathrm{H}$ & 3.9920000000 & 2.0526820000 & -0.0634860000 \\
$\mathrm{H}$ & 4.5116110000 & 1.6438310000 & 1.5923160000 \\
$\mathrm{H}$ & 2.7923050000 & 1.4846820000 & 1.1387560000 \\
$\mathrm{H}$ & -5.4282110000 & 1.2818050000 & 0.4813550000 \\
$\mathrm{H}$ & -5.4481410000 & -0.2957580000 & 1.3291960000 \\
$\mathrm{H}$ & -6.1962710000 & -0.1339350000 & -0.2791550000 \\
$\mathrm{H}$ & -2.7674760000 & 1.4704740000 & 0.8702930000 \\
$\mathrm{H}$ & -2.7666580000 & -0.0940020000 & 1.7189010000 \\
$\mathrm{H}$ & 3.1534400000 & 0.0882890000 & -1.8710270000 \\
$\mathrm{H}$ & 3.0207760000 & -1.6128280000 & -1.3907160000
\end{tabular}




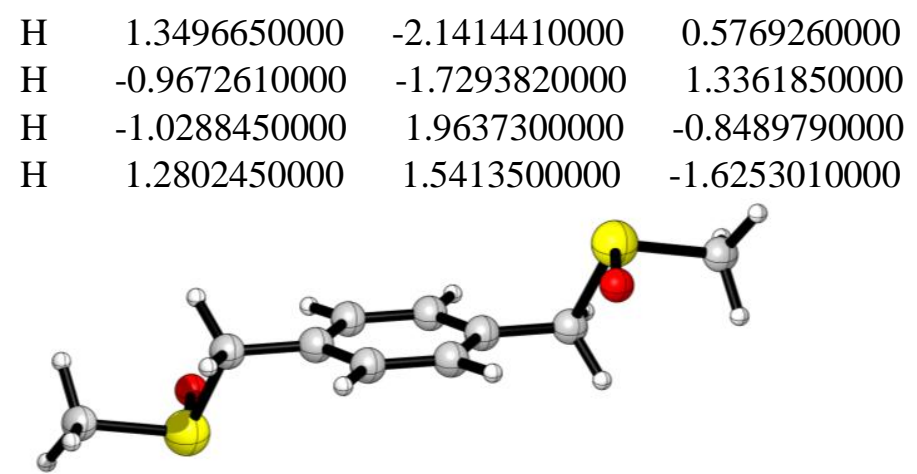

\begin{tabular}{lccc}
\multicolumn{5}{c}{ meso-1,4-bis((methylsulfinyl)methyl)benzene } \\
Energy: -1335.554156 & & \\
S & 3.9555860000 & -0.0739700000 & 0.5349590000 \\
S & -3.9557540000 & 0.0742280000 & -0.5347250000 \\
O & -4.0030390000 & 1.6506770000 & 0.0409770000 \\
O & 4.0038360000 & -1.6505070000 & -0.0403520000 \\
C & 5.5074960000 & 0.7166980000 & -0.1617960000 \\
C & -5.5076940000 & -0.7159680000 & 0.1625530000 \\
C & -2.7177630000 & -0.7905930000 & 0.6374060000 \\
C & 2.7177060000 & 0.7901840000 & -0.6379380000 \\
C & 1.3143540000 & 0.3858300000 & -0.3108920000 \\
$\mathrm{C}$ & 0.9026870000 & -0.9399340000 & -0.5088430000 \\
$\mathrm{C}$ & -0.4018560000 & -1.3168700000 & -0.2015330000 \\
$\mathrm{C}$ & -1.3144430000 & -0.3862680000 & 0.3103020000 \\
$\mathrm{C}$ & -0.9027680000 & 0.9395170000 & 0.5082620000 \\
$\mathrm{C}$ & 0.4017690000 & 1.3164480000 & 0.2009700000 \\
$\mathrm{H}$ & 5.4821560000 & 1.7894630000 & 0.0337190000 \\
$\mathrm{H}$ & 5.5486850000 & 0.4996400000 & -1.2299800000 \\
$\mathrm{H}$ & 6.3501310000 & 0.2490060000 & 0.3452770000 \\
$\mathrm{H}$ & -5.5478720000 & -0.4997120000 & 1.2309400000 \\
$\mathrm{H}$ & -5.4831020000 & -1.7885910000 & -0.0337910000 \\
$\mathrm{H}$ & -6.3504190000 & -0.2474100000 & -0.3435670000 \\
$\mathrm{H}$ & -3.0294390000 & -0.4545510000 & 1.6302510000 \\
$\mathrm{H}$ & -2.8818350000 & -1.8651520000 & 0.5283420000 \\
$\mathrm{H}$ & 2.8815760000 & 1.8648060000 & -0.5293400000 \\
$\mathrm{H}$ & 3.0296280000 & 0.4535980000 & -1.6305110000 \\
$\mathrm{H}$ & 1.6217180000 & -1.6691430000 & -0.8665840000 \\
$\mathrm{H}$ & -0.7093410000 & -2.3470580000 & -0.3539620000 \\
$\mathrm{H}$ & -1.6217600000 & 1.6687130000 & 0.8661220000 \\
$\mathrm{H}$ & 0.7092660000 & 2.3466330000 & 0.3533990000
\end{tabular}




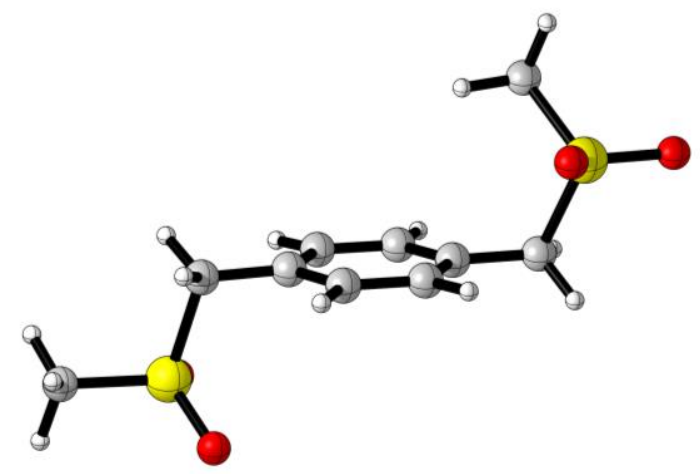

\section{1,4-bis((methylsulfonyl)methyl)benzene}

Energy: -1485.718602

$\begin{array}{lrrr}\mathrm{S} & 4.0225890000 & -0.1305790000 & 0.0215330000 \\ \mathrm{~S} & -3.8888310000 & 0.0017180000 & -0.1967970000 \\ \mathrm{O} & 3.7866420000 & -1.6351180000 & 0.6114310000 \\ \mathrm{O} & 5.4294770000 & 0.2544480000 & -0.7093240000 \\ \mathrm{O} & -3.6697030000 & -1.2584630000 & -1.2080480000 \\ \mathrm{O} & -3.8332140000 & 1.5370230000 & -0.7514830000 \\ \mathrm{C} & 3.7286520000 & 1.0735950000 & 1.4077810000 \\ \mathrm{C} & -5.5218340000 & -0.2309680000 & 0.6641460000 \\ \mathrm{C} & -2.5961790000 & -0.1329040000 & 1.2007020000 \\ \mathrm{C} & 2.6373350000 & 0.2287750000 & -1.2341710000 \\ \mathrm{C} & 1.2874060000 & 0.1346580000 & -0.6095090000 \\ \mathrm{C} & 0.7488710000 & -1.1155890000 & -0.2753780000 \\ \mathrm{C} & -0.5055120000 & -1.1988920000 & 0.3202330000 \\ \mathrm{C} & -1.2332670000 & -0.0376030000 & 0.6055520000 \\ \mathrm{C} & -0.7008910000 & 1.2129600000 & 0.2653150000 \\ \mathrm{C} & 0.5525170000 & 1.2957680000 & -0.3326620000 \\ \mathrm{H} & 3.8531580000 & 2.0761990000 & 1.0022370000 \\ \mathrm{H} & 4.4863250000 & 0.8522970000 & 2.1573690000 \\ \mathrm{H} & 2.7248910000 & 0.8988350000 & 1.7906210000 \\ \mathrm{H} & -5.6210400000 & 0.5565700000 & 1.4093080000 \\ \mathrm{H} & -5.5282920000 & -1.2278950000 & 1.1014880000 \\ \mathrm{H} & -6.2816640000 & -0.1367150000 & -0.1099120000 \\ \mathrm{H} & -2.8455740000 & 0.6962570000 & 1.8649670000 \\ \mathrm{H} & -2.7958070000 & -1.0948330000 & 1.6751980000 \\ \mathrm{H} & 2.8834620000 & 1.2162320000 & -1.6273930000 \\ \mathrm{H} & 2.8273030000 & -0.5306000000 & -1.9949840000 \\ \mathrm{H} & 1.3225870000 & -2.0145740000 & -0.4669030000 \\ \mathrm{H} & -0.9266720000 & -2.1702470000 & 0.5535210000 \\ \mathrm{H} & -1.2817090000 & 2.1113820000 & 0.4398330000 \\ \mathrm{H} & 0.9547030000 & 2.2669550000 & -0.6037720000\end{array}$




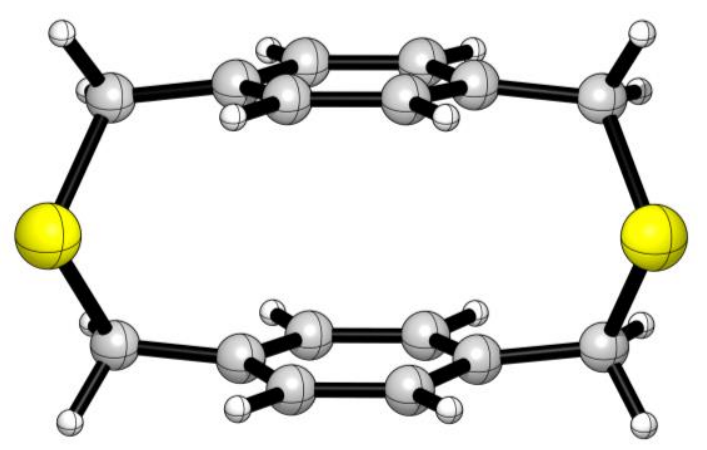

dt[3.3]pCpTA-S boat

Energy: -1415.30069914

$\begin{array}{lrrr}\text { S } & -3.5638410000 & -0.0238030000 & -0.7712990000 \\ \mathrm{~S} & 3.5637990000 & -0.0238280000 & -0.7713280000 \\ \mathrm{C} & -2.9087480000 & -1.4818130000 & 0.2487920000 \\ \mathrm{C} & -2.9088360000 & 1.4866410000 & 0.1662200000 \\ \mathrm{C} & 0.6944510000 & 1.6326660000 & -1.0566110000 \\ \mathrm{C} & -0.6943390000 & 1.6326730000 & -1.0566370000 \\ \mathrm{C} & -1.4107670000 & 1.6482390000 & 0.1477810000 \\ \mathrm{C} & -0.6976980000 & 1.7580860000 & 1.3429970000 \\ \mathrm{C} & 0.6977360000 & 1.7581210000 & 1.3430200000 \\ \mathrm{C} & 1.4108350000 & 1.6482600000 & 0.1478250000 \\ \mathrm{C} & 2.9088850000 & 1.4865600000 & 0.1663010000 \\ \mathrm{C} & 2.9087080000 & -1.4818690000 & 0.2487520000 \\ \mathrm{C} & -0.6975230000 & -1.5460020000 & 1.4480080000 \\ \mathrm{C} & -1.4110970000 & -1.6349330000 & 0.2520890000 \\ \mathrm{C} & -0.6946620000 & -1.8101020000 & -0.9407540000 \\ \mathrm{C} & 0.6945990000 & -1.8100980000 & -0.9407690000 \\ \mathrm{C} & 1.4110530000 & -1.6349660000 & 0.2520690000 \\ \mathrm{C} & 0.6974970000 & -1.5460490000 & 1.4479990000 \\ \mathrm{H} & 1.2320750000 & 1.5470800000 & -1.9952410000 \\ \mathrm{H} & -1.2326390000 & 1.7846950000 & 2.2880960000 \\ \mathrm{H} & 1.2319020000 & -1.4192320000 & 2.3850310000 \\ \mathrm{H} & -1.2336240000 & -1.8826960000 & -1.8800790000 \\ \mathrm{H} & 3.2981680000 & -1.3809880000 & 1.2651380000 \\ \mathrm{H} & 3.4052370000 & -2.3353030000 & -0.2220640000 \\ \mathrm{H} & -3.2981960000 & -1.3809370000 & 1.2651860000 \\ \mathrm{H} & -3.4052820000 & -2.3352530000 & -0.2220100000 \\ \mathrm{H} & 3.2872470000 & 1.4380980000 & 1.1908360000 \\ \mathrm{H} & 3.4150700000 & 2.3129340000 & -0.3418610000 \\ \mathrm{H} & -3.2872310000 & 1.4382630000 & 1.1907420000 \\ \mathrm{H} & -3.4149920000 & 2.3129820000 & -0.3420280000 \\ \mathrm{H} & -1.2319050000 & -1.4191680000 & 2.3850500000 \\ \mathrm{H} & 1.2335500000 & -1.8826960000 & -1.8800990000 \\ \mathrm{H} & -1.2319210000 & 1.5471130000 & -1.9952900000 \\ \mathrm{H} & 1.2326450000 & 1.7847160000 & 2.2881360000\end{array}$




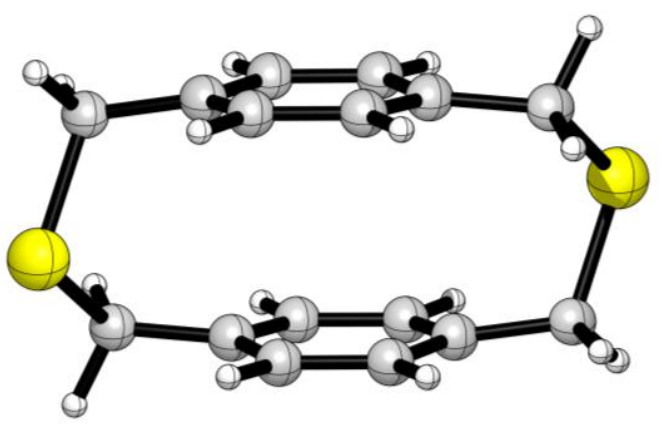

dt[3.3]pCpTA-S chair

Energy: -1415.30051718

$\begin{array}{lrrr}\text { S } & -3.6729000000 & -0.1069700000 & 0.4197440000 \\ \mathrm{~S} & 3.6728680000 & 0.1069360000 & -0.4198300000 \\ \mathrm{C} & -2.9861480000 & 1.3603780000 & -0.5731150000 \\ \mathrm{C} & -2.7587730000 & -1.5879000000 & -0.3183170000 \\ \mathrm{C} & 0.9880230000 & -2.0234550000 & -0.8325890000 \\ \mathrm{C} & -0.3883720000 & -2.0560620000 & -1.0393290000 \\ \mathrm{C} & -1.2774790000 & -1.6669430000 & -0.0283440000 \\ \mathrm{C} & -0.7537940000 & -1.3277040000 & 1.2214330000 \\ \mathrm{C} & 0.6226150000 & -1.2899660000 & 1.4256420000 \\ \mathrm{C} & 1.5127830000 & -1.5930240000 & 0.3915350000 \\ \mathrm{C} & 2.9861530000 & -1.3603500000 & 0.5731400000 \\ \mathrm{C} & 2.7587940000 & 1.5878720000 & 0.3182790000 \\ \mathrm{C} & -0.6226250000 & 1.2902590000 & -1.4256830000 \\ \mathrm{C} & -1.5127790000 & 1.5930320000 & -0.3914820000 \\ \mathrm{C} & -0.9880050000 & 2.0231940000 & 0.8327310000 \\ \mathrm{C} & 0.3883950000 & 2.0558010000 & 1.0394460000 \\ \mathrm{C} & 1.2774900000 & 1.6669490000 & 0.0283480000 \\ \mathrm{C} & 0.7537870000 & 1.3279940000 & -1.2214980000 \\ \mathrm{H} & -0.7767100000 & -2.3409250000 & -2.0132110000 \\ \mathrm{H} & 1.0105160000 & -0.9633250000 & 2.3861360000 \\ \mathrm{H} & -1.6624220000 & 2.2682230000 & 1.6476940000 \\ \mathrm{H} & 1.4252490000 & 1.0187310000 & -2.0148750000 \\ \mathrm{H} & 3.2369690000 & -1.1960590000 & 1.6239820000 \\ \mathrm{H} & 3.5865210000 & -2.1951380000 & 0.2017620000 \\ \mathrm{H} & -3.2988140000 & -2.4358690000 & 0.1149090000 \\ \mathrm{H} & -2.9414600000 & -1.5940800000 & -1.3965580000 \\ \mathrm{H} & 2.9414940000 & 1.5939910000 & 1.3965180000 \\ \mathrm{H} & 3.2988470000 & 2.4358530000 & -0.1149090000 \\ \mathrm{H} & -3.5865030000 & 2.1951380000 & -0.2016540000 \\ \mathrm{H} & -3.2369720000 & 1.1961950000 & -1.6239730000 \\ \mathrm{H} & -1.4252660000 & -1.0182260000 & 2.0147190000 \\ \mathrm{H} & 1.6624510000 & -2.2686930000 & -1.6474800000 \\ \mathrm{H} & -1.0105380000 & 0.9638390000 & -2.3862480000 \\ \mathrm{H} & 0.7767470000 & 2.3404470000 & 2.0133860000\end{array}$




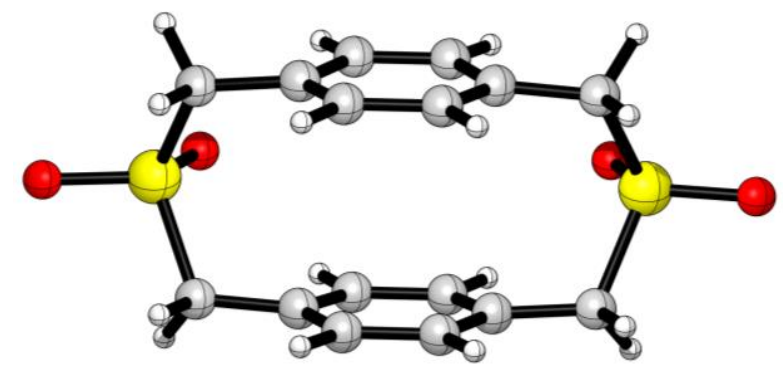

$\mathrm{dt}[3.3] \mathrm{pCp}-\mathrm{SO}_{2}$ boat

Energy: -1715.64174329

$\begin{array}{lrrr}\text { S } & -3.5645410000 & -0.0005230000 & -0.4290280000 \\ \text { S } & 3.5645740000 & 0.0006180000 & -0.4290100000 \\ \mathrm{O} & 3.0267960000 & 0.0015080000 & -1.9718340000 \\ \mathrm{O} & -3.0265760000 & -0.0008090000 & -1.9717890000 \\ \mathrm{O} & -5.1664340000 & -0.0018920000 & -0.1253370000 \\ \mathrm{O} & 5.1664360000 & 0.0015710000 & -0.1251480000 \\ \mathrm{C} & 2.8929370000 & -1.5778620000 & 0.4131590000 \\ \mathrm{C} & -0.6939640000 & -1.6725480000 & 1.6122860000 \\ \mathrm{C} & 0.6968650000 & -1.6730260000 & 1.6117290000 \\ \mathrm{C} & 1.4041840000 & -1.6855570000 & 0.4046930000 \\ \mathrm{C} & 0.6961060000 & -1.7628300000 & -0.8028090000 \\ \mathrm{C} & -0.6952760000 & -1.7624250000 & -0.8022550000 \\ \mathrm{C} & -1.4023050000 & -1.6846660000 & 0.4058320000 \\ \mathrm{C} & -2.8910780000 & -1.5767260000 & 0.4157830000 \\ \mathrm{C} & -2.8929870000 & 1.5778640000 & 0.4135420000 \\ \mathrm{C} & 2.8910610000 & 1.5767640000 & 0.4160360000 \\ \mathrm{C} & -0.6961740000 & 1.7632530000 & -0.8023340000 \\ \mathrm{C} & -1.4042270000 & 1.6854500000 & 0.4051410000 \\ \mathrm{C} & -0.6968800000 & 1.6723670000 & 1.6121670000 \\ \mathrm{C} & 0.6939530000 & 1.6718700000 & 1.6126870000 \\ \mathrm{C} & 1.4022640000 & 1.6845230000 & 0.4062070000 \\ \mathrm{C} & 0.6951970000 & 1.7628690000 & -0.8018160000 \\ \mathrm{H} & 1.2378820000 & 1.7576840000 & -1.7400920000 \\ \mathrm{H} & -1.2342490000 & 1.6295720000 & 2.5545110000 \\ \mathrm{H} & 1.2342630000 & -1.6306800000 & 2.5540770000 \\ \mathrm{H} & -1.2379890000 & -1.7568220000 & -1.7405180000 \\ \mathrm{H} & 3.3343850000 & -1.5482020000 & 1.4103290000 \\ \mathrm{H} & 3.3982620000 & -2.3374300000 & -0.1884890000 \\ \mathrm{H} & -3.3312230000 & -1.5448660000 & 1.4134740000 \\ \mathrm{H} & -3.3971580000 & -2.3378340000 & -0.1833050000 \\ \mathrm{H} & 3.3312690000 & 1.5449090000 & 1.4136960000 \\ \mathrm{H} & 3.3970130000 & 2.3379280000 & -0.1830800000 \\ \mathrm{H} & -3.3344890000 & 1.5480590000 & 1.4106820000 \\ \mathrm{H} & -3.3982380000 & 2.3375080000 & -0.1880610000 \\ \mathrm{H} & -1.2305620000 & -1.6297540000 & 2.5550650000 \\ \mathrm{H} & 1.2380490000 & -1.7574790000 & -1.7415190000 \\ \mathrm{H} & -1.2381470000 & 1.7582990000 & -1.7410290000\end{array}$




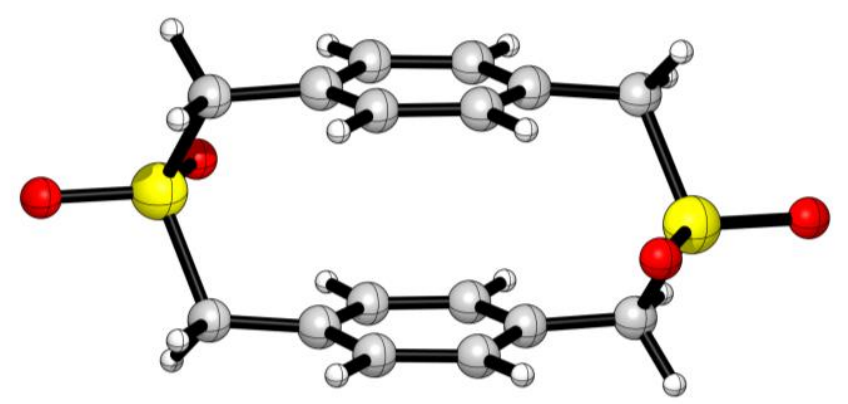

dt[3.3]pCp-SO $\mathrm{O}_{2}$ chair

Energy: -1715.64312311

$\begin{array}{lrrr}\mathrm{S} & -3.6637030000 & 0.0018130000 & -0.0780500000 \\ \mathrm{~S} & 3.6637050000 & -0.0018200000 & 0.0780500000 \\ \mathrm{O} & 3.4609710000 & 0.0058290000 & 1.7006730000 \\ \mathrm{O} & -3.4609660000 & -0.0058560000 & -1.7006720000 \\ \mathrm{O} & -5.1688910000 & 0.0085080000 & 0.5478550000 \\ \mathrm{O} & 5.1688940000 & -0.0085120000 & -0.5478550000 \\ \mathrm{C} & 2.8248720000 & -1.5783900000 & -0.5994720000 \\ \mathrm{C} & -0.4339670000 & -1.6927090000 & 1.3290710000 \\ \mathrm{C} & 0.9286280000 & -1.7113690000 & 1.0502670000 \\ \mathrm{C} & 1.3698820000 & -1.6815160000 & -0.2791280000 \\ \mathrm{C} & 0.4270260000 & -1.7189880000 & -1.3132400000 \\ \mathrm{C} & -0.9355700000 & -1.7368860000 & -1.0339600000 \\ \mathrm{C} & -1.3769590000 & -1.6808630000 & 0.2945450000 \\ \mathrm{C} & -2.8317400000 & -1.5730710000 & 0.6126030000 \\ \mathrm{C} & -2.8248780000 & 1.5783910000 & 0.5994580000 \\ \mathrm{C} & 2.8317430000 & 1.5730730000 & -0.6125870000 \\ \mathrm{C} & -0.4270360000 & 1.7190290000 & 1.3132360000 \\ \mathrm{C} & -1.3698860000 & 1.6815220000 & 0.2791190000 \\ \mathrm{C} & -0.9286230000 & 1.7113490000 & -1.0502740000 \\ \mathrm{C} & 0.4339730000 & 1.6926880000 & -1.3290680000 \\ \mathrm{C} & 1.3769600000 & 1.6808640000 & -0.2945360000 \\ \mathrm{C} & 0.9355610000 & 1.7369280000 & 1.0339640000 \\ \mathrm{H} & -0.7589620000 & 1.7112570000 & 2.3467910000 \\ \mathrm{H} & 0.7659370000 & 1.6637510000 & -2.3622360000 \\ \mathrm{H} & -1.6598290000 & -1.7184130000 & -1.8400810000 \\ \mathrm{H} & 1.6529080000 & -1.6729870000 & 1.8556690000 \\ \mathrm{H} & 3.0528760000 & 1.5381140000 & -1.6803060000 \\ \mathrm{H} & 3.4517190000 & 2.3355670000 & -0.1344430000 \\ \mathrm{H} & -3.4433030000 & 2.3393850000 & 0.1168040000 \\ \mathrm{H} & -3.0445910000 & 1.5524460000 & 1.6677510000 \\ \mathrm{H} & 3.0445830000 & -1.5524380000 & -1.6677650000 \\ \mathrm{H} & 3.4432950000 & -2.3393910000 & -0.1168240000 \\ \mathrm{H} & -3.4517170000 & -2.3355670000 & 0.1344660000 \\ \mathrm{H} & -3.0528700000 & -1.5381020000 & 1.6803230000 \\ \mathrm{H} & -1.6528980000 & 1.6729440000 & -1.8556800000\end{array}$




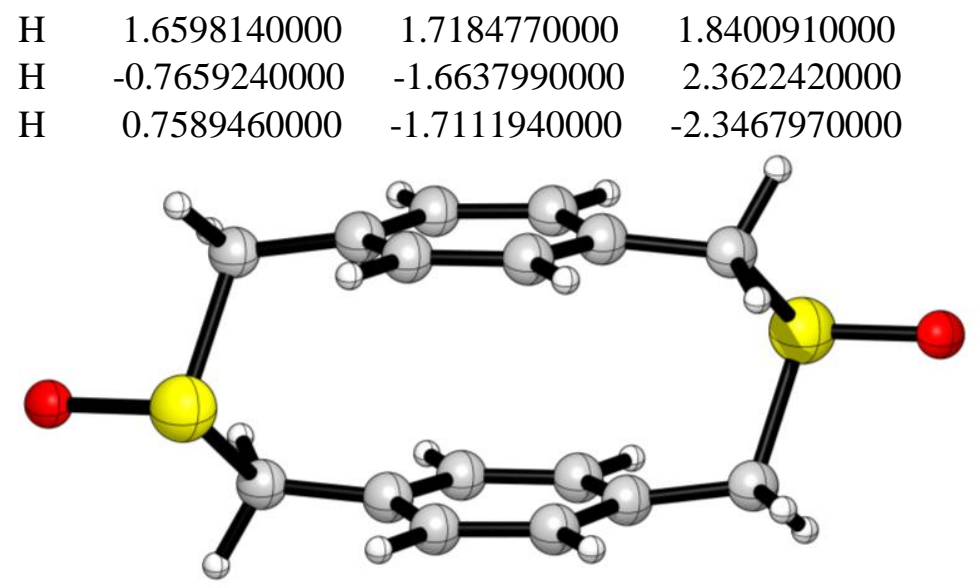

dt[3.3]pCp-SO chair trans out Energy: -1565.46093418

$\begin{array}{lrrc}\text { S } & -3.6303390000 & -0.0112770000 & 0.4771490000 \\ \text { S } & 3.6303610000 & 0.0112880000 & -0.4771300000 \\ \mathrm{O} & -5.2026430000 & -0.1356660000 & -0.0766620000 \\ \mathrm{O} & 5.2026460000 & 0.1356740000 & 0.0767260000 \\ \mathrm{C} & -2.9331540000 & 1.4476700000 & -0.5534050000 \\ \mathrm{C} & 0.4379450000 & 1.9672550000 & 1.1089210000 \\ \mathrm{C} & -0.9367560000 & 1.9803910000 & 0.8922870000 \\ \mathrm{C} & -1.4636400000 & 1.6403610000 & -0.3608230000 \\ \mathrm{C} & -0.5746130000 & 1.3931890000 & -1.4114120000 \\ \mathrm{C} & 0.8006530000 & 1.4095630000 & -1.2024630000 \\ \mathrm{C} & 1.3248240000 & 1.6438340000 & 0.0739320000 \\ \mathrm{C} & 2.7945300000 & 1.5154860000 & 0.3512370000 \\ \mathrm{C} & -2.7945360000 & -1.5154850000 & -0.3512310000 \\ \mathrm{C} & 2.9331510000 & -1.4476740000 & 0.5533850000 \\ \mathrm{C} & -0.4379560000 & -1.9672090000 & -1.1089480000 \\ \mathrm{C} & -1.3248330000 & -1.6438610000 & -0.0739350000 \\ \mathrm{C} & -0.8006580000 & -1.4096580000 & 1.2024710000 \\ \mathrm{C} & 0.5746090000 & -1.3932400000 & 1.4114040000 \\ \mathrm{C} & 1.4636340000 & -1.6403400000 & 0.3607960000 \\ \mathrm{C} & 0.9367480000 & -1.9803160000 & -0.8923280000 \\ \mathrm{H} & -0.8265160000 & -2.1762960000 & -2.1008020000 \\ \mathrm{H} & 0.9630960000 & -1.1469520000 & 2.3945600000 \\ \mathrm{H} & -1.6079520000 & 2.2043060000 & 1.7159640000 \\ \mathrm{H} & 1.4664130000 & 1.1827230000 & -2.0285230000 \\ \mathrm{H} & 3.2192120000 & -1.2281570000 & 1.5844230000 \\ \mathrm{H} & 3.5406630000 & -2.2786270000 & 0.1875650000 \\ \mathrm{H} & -3.3881710000 & -2.3362490000 & 0.0608150000 \\ \mathrm{H} & -3.0247210000 & -1.4274960000 & -1.4158820000 \\ \mathrm{H} & 3.0247110000 & 1.4275140000 & 1.4158900000 \\ \mathrm{H} & 3.3881580000 & 2.3362520000 & -0.0608170000 \\ \mathrm{H} & -3.5406720000 & 2.2786290000 & -0.1876130000 \\ \mathrm{H} & -3.2192100000 & 1.2281180000 & -1.5844370000\end{array}$




$\begin{array}{rrrr}\mathrm{H} & -1.4664180000 & -1.1828600000 & 2.0285430000 \\ \mathrm{H} & 1.6079410000 & -2.2041820000 & -1.7160200000 \\ \mathrm{H} & -0.9630970000 & 1.1468500000 & -2.3945560000 \\ \mathrm{H} & 0.8265020000 & 2.1763960000 & 2.1007650000\end{array}$

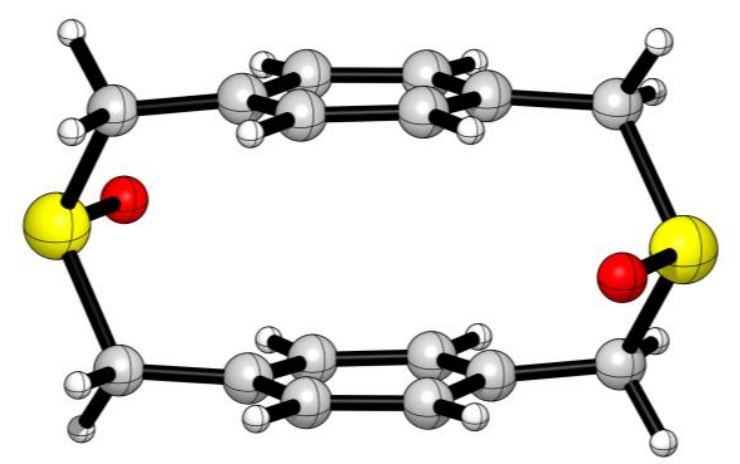

dt[3.3]pCp-SO chair trans in

Energy: -1565.46913369

$\begin{array}{lrrr}\text { S } & -3.7421240000 & -0.0003880000 & 0.1075900000 \\ \text { S } & 3.7421200000 & 0.0003580000 & -0.1076310000 \\ \text { O } & -3.4760950000 & -0.0006540000 & -1.5495090000 \\ \text { O } & 3.4761450000 & 0.0005700000 & 1.5494750000 \\ \mathrm{C} & -2.7842360000 & -1.5240000000 & 0.7908320000 \\ \mathrm{C} & 0.3417810000 & -1.6664280000 & -1.3445230000 \\ \mathrm{C} & -1.0003260000 & -1.6879340000 & -0.9782550000 \\ \mathrm{C} & -1.3525760000 & -1.6377220000 & 0.3778870000 \\ \mathrm{C} & -0.3409390000 & -1.6650050000 & 1.3452590000 \\ \mathrm{C} & 1.0011520000 & -1.6867960000 & 0.9790560000 \\ \mathrm{C} & 1.3534060000 & -1.6380360000 & -0.3771960000 \\ \mathrm{C} & 2.7849810000 & -1.5242070000 & -0.7902340000 \\ \mathrm{C} & -2.7850150000 & 1.5242100000 & 0.7901690000 \\ \mathrm{C} & 2.7842480000 & 1.5240380000 & -0.7907610000 \\ \mathrm{C} & -0.3418210000 & 1.6662280000 & 1.3445340000 \\ \mathrm{C} & -1.3534220000 & 1.6380500000 & 0.3771780000 \\ \mathrm{C} & -1.0011300000 & 1.6870470000 & -0.9790590000 \\ \mathrm{C} & 0.3409710000 & 1.6652670000 & -1.3452290000 \\ \mathrm{C} & 1.3525820000 & 1.6377380000 & -0.3778360000 \\ \mathrm{C} & 1.0002970000 & 1.6877090000 & 0.9783040000 \\ \mathrm{H} & -0.6038520000 & 1.6388820000 & 2.3982720000 \\ \mathrm{H} & 0.6030530000 & 1.6372400000 & -2.3989370000 \\ \mathrm{H} & -1.7779180000 & -1.6461750000 & -1.7311210000 \\ \mathrm{H} & 1.7786820000 & -1.6437290000 & 1.7319600000 \\ \mathrm{H} & 2.9109840000 & 1.4716510000 & -1.8752890000 \\ \mathrm{H} & 3.4013010000 & 2.3351610000 & -0.3959790000 \\ \mathrm{H} & -3.4024780000 & 2.3348550000 & 0.3950420000 \\ \mathrm{H} & -2.9118450000 & 1.4720950000 & 1.8746860000 \\ \mathrm{H} & 2.9117900000 & -1.4720950000 & -1.8747540000 \\ \mathrm{H} & 3.4024430000 & -2.3348540000 & -0.3951130000 \\ \mathrm{H} & -3.4012900000 & -2.3351770000 & 0.3961640000\end{array}$




$\begin{array}{rrrr}\mathrm{H} & -2.9109410000 & -1.4714840000 & 1.8753580000 \\ \mathrm{H} & -1.7786440000 & 1.6441370000 & -1.7319940000 \\ \mathrm{H} & 1.7778660000 & 1.6457700000 & 1.7311840000 \\ \mathrm{H} & -0.6029950000 & -1.6368010000 & 2.3989690000 \\ \mathrm{H} & 0.6037840000 & -1.6392660000 & -2.3982720000\end{array}$

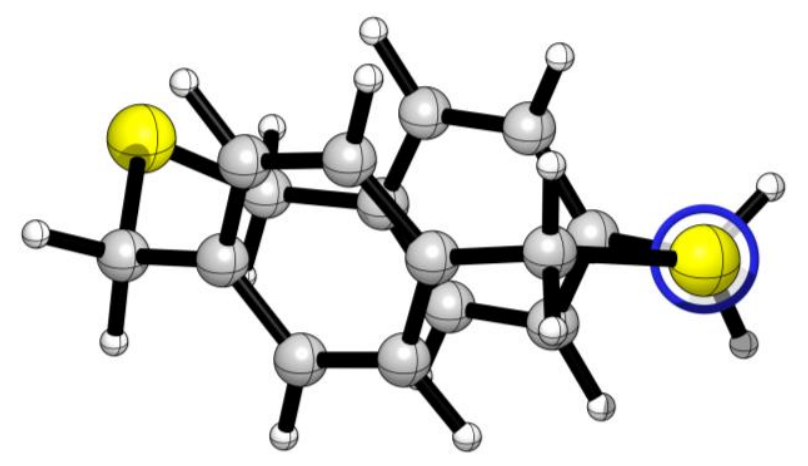

\section{dt[3.3]pCp-S TS}

Energy: -1415.30069914

$\begin{array}{lrrr}\mathrm{S} & 3.6264250000 & -0.1416420000 & -0.5703230000 \\ \mathrm{~S} & -3.8377580000 & -0.0519520000 & -0.1762770000 \\ \mathrm{C} & 2.9124330000 & -1.2724930000 & 0.6845650000 \\ \mathrm{C} & 2.9362930000 & 1.4835580000 & -0.0907150000 \\ \mathrm{C} & -0.6751920000 & 2.1404590000 & 0.9171490000 \\ \mathrm{C} & 0.7198020000 & 2.1453970000 & 0.9140920000 \\ \mathrm{C} & 1.4305240000 & 1.6063470000 & -0.1564690000 \\ \mathrm{C} & 0.7128940000 & 1.1377620000 & -1.2632240000 \\ \mathrm{C} & -0.6742130000 & 1.1375700000 & -1.2618460000 \\ \mathrm{C} & -1.3884020000 & 1.5988020000 & -0.1513950000 \\ \mathrm{C} & -2.8895280000 & 1.5516840000 & -0.1116640000 \\ \mathrm{C} & -2.8385740000 & -1.6231910000 & -0.1006250000 \\ \mathrm{C} & 0.5493000000 & -1.0462690000 & 1.5277190000 \\ \mathrm{C} & -0.8271750000 & -1.1021020000 & 1.3383620000 \\ \mathrm{C} & -1.3579260000 & -1.6139560000 & 0.1549860000 \\ \mathrm{C} & -0.4803810000 & -2.1516630000 & -0.7921350000 \\ \mathrm{C} & 0.8962490000 & -2.1044000000 & -0.5976650000 \\ \mathrm{C} & 1.4291970000 & -1.5067680000 & 0.5462470000 \\ \mathrm{H} & -3.3452280000 & -2.1893860000 & 0.6864030000 \\ \mathrm{H} & -3.0325820000 & -2.1253660000 & -1.0508100000 \\ \mathrm{H} & 3.4766720000 & -2.2001780000 & 0.5397700000 \\ \mathrm{H} & 3.1550200000 & -0.8938470000 & 1.6832050000 \\ \mathrm{H} & 3.4187600000 & 2.1769010000 & -0.7895380000 \\ \mathrm{H} & 3.2917680000 & 1.7404150000 & 0.9129590000 \\ \mathrm{H} & -3.2962280000 & 2.1019250000 & -0.9654180000 \\ \mathrm{H} & -3.2368070000 & 2.0444780000 & 0.7988920000 \\ \mathrm{H} & 0.9480150000 & -0.5885100000 & 2.4307400000 \\ \mathrm{H} & -1.4970140000 & -0.6911320000 & 2.0914530000 \\ \mathrm{H} & -0.8796130000 & -2.5779880000 & -1.7098700000\end{array}$




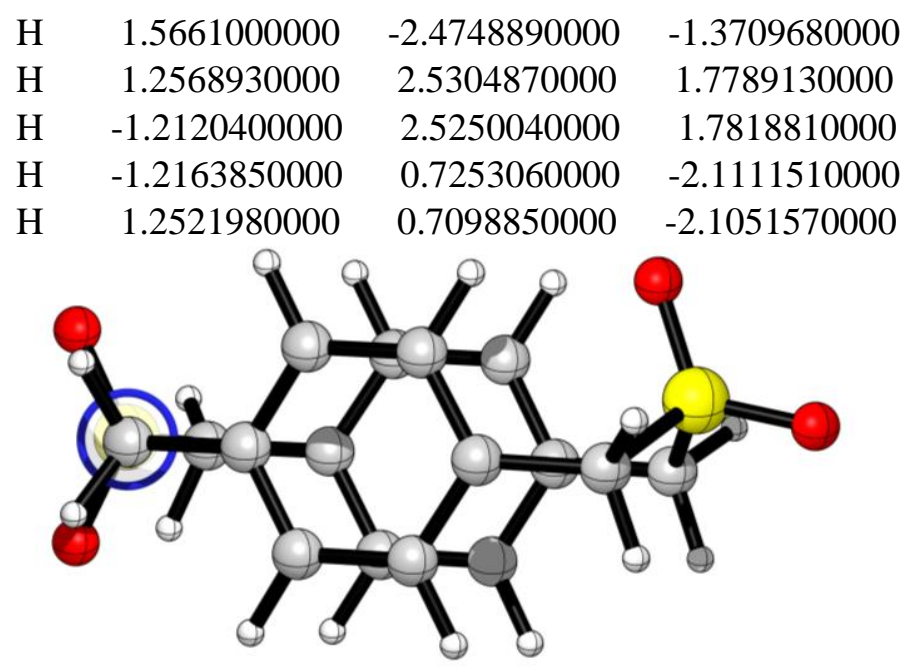

dt[3.3]pCp-SO $\mathrm{OS}_{2}$

Energy: -1715.62924365

$\begin{array}{lrrr}\text { S } & -3.4856310000 & -0.0333720000 & -0.1979860000 \\ \text { S } & 3.5941220000 & -0.0895420000 & -0.2656030000 \\ \text { O } & 3.2044840000 & -0.0898170000 & -1.6768420000 \\ \text { O } & -4.1141700000 & -0.1939360000 & -1.5104540000 \\ \text { O } & -4.2703390000 & -0.2232610000 & 1.0221010000 \\ \text { O } & 5.0077060000 & -0.1429370000 & 0.1077190000 \\ \mathrm{C} & 2.8172970000 & -1.5323780000 & 0.5246250000 \\ \mathrm{C} & -0.8829440000 & -1.5422710000 & 1.3410470000 \\ \mathrm{C} & 0.5002450000 & -1.5972910000 & 1.4835650000 \\ \mathrm{C} & 1.3261590000 & -1.6375420000 & 0.3603970000 \\ \mathrm{C} & 0.7409600000 & -1.7204540000 & -0.9082220000 \\ \mathrm{C} & -0.6405290000 & -1.6655030000 & -1.0506660000 \\ \mathrm{C} & -1.4633720000 & -1.5266840000 & 0.0728740000 \\ \mathrm{C} & -2.9437820000 & -1.3035320000 & -0.0689850000 \\ \mathrm{C} & -2.8229220000 & 1.6583010000 & -0.1035440000 \\ \mathrm{C} & 2.9380860000 & 1.4294670000 & 0.4907630000 \\ \mathrm{C} & -0.4985650000 & 1.8082650000 & -1.0908220000 \\ \mathrm{C} & -1.3291230000 & 1.7630570000 & 0.0347240000 \\ \mathrm{C} & -0.7482770000 & 1.7603640000 & 1.3028370000 \\ \mathrm{C} & 0.6348910000 & 1.7053470000 & 1.4455550000 \\ \mathrm{C} & 1.4603770000 & 1.6522030000 & 0.3225450000 \\ \mathrm{C} & 0.8829140000 & 1.7533160000 & -0.9482790000 \\ \mathrm{H} & 1.5167950000 & 1.7260140000 & -1.8296360000 \\ \mathrm{H} & -1.3805800000 & 1.7515140000 & 2.1881420000 \\ \mathrm{H} & 0.9398960000 & -1.5589370000 & 2.4780840000 \\ \mathrm{H} & -1.0824310000 & -1.6676020000 & -2.0425670000 \\ \mathrm{H} & 3.1236370000 & -1.5005460000 & 1.5742510000 \\ \mathrm{H} & 3.3619650000 & -2.3493150000 & 0.0356360000 \\ \mathrm{H} & -3.4538620000 & -1.2389850000 & 0.8964570000 \\ \mathrm{H} & -3.4398200000 & -2.0793460000 & -0.6649740000 \\ \mathrm{H} & 3.2415830000 & 1.3966650000 & 1.5411140000\end{array}$




$\begin{array}{rrrr}\mathrm{H} & 3.5471500000 & 2.1879120000 & -0.0161350000 \\ \mathrm{H} & -3.3564670000 & 2.1439590000 & 0.7184980000 \\ \mathrm{H} & -3.1973320000 & 2.0758060000 & -1.0462360000 \\ \mathrm{H} & -1.5114890000 & -1.4614040000 & 2.2254250000 \\ \mathrm{H} & 1.3741260000 & -1.7652400000 & -1.7893770000 \\ \mathrm{H} & -0.9397410000 & 1.8236490000 & -2.0830260000 \\ \mathrm{H} & 1.0707780000 & 1.6540860000 & 2.4410980000\end{array}$

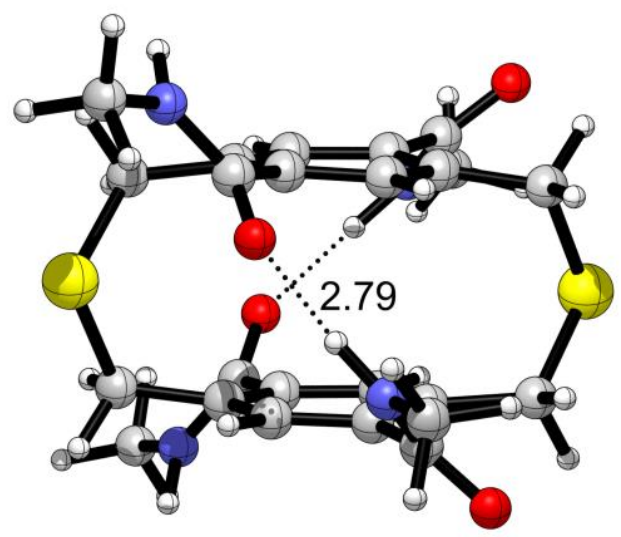

$\left(S_{\mathbf{p}}\right)-1$ chair anti-

Energy: -2246.86952385

$\begin{array}{lrrr}\text { S } & -1.7202140000 & 3.1712210000 & 0.0761760000 \\ \text { S } & 1.7876480000 & -3.3278410000 & -0.1177440000 \\ \text { O } & 3.0596930000 & -1.9416510000 & 2.7898190000 \\ \text { O } & -1.9772740000 & -2.8580550000 & -2.9284920000 \\ \text { O } & 3.0909080000 & 1.7553650000 & -0.5491260000 \\ \text { O } & -3.5874270000 & 0.0229860000 & 0.7442830000 \\ \mathrm{~N} & 3.6915360000 & -0.2583180000 & 1.3787990000 \\ \mathrm{~N} & -3.2465390000 & 1.3675780000 & 2.5513470000 \\ \mathrm{~N} & 2.3441650000 & 2.7757160000 & -2.4441500000 \\ \mathrm{~N} & -3.2413800000 & -1.7319440000 & -1.3920630000 \\ \mathrm{C} & 5.1026500000 & -0.5749800000 & 1.5213870000 \\ \mathrm{C} & 2.7625610000 & -0.9914350000 & 2.0284780000 \\ \mathrm{C} & -4.6256640000 & 1.8256590000 & 2.6258570000 \\ \mathrm{C} & 3.4412670000 & 3.7316020000 & -2.5052050000 \\ \mathrm{C} & -0.5864140000 & 2.6783220000 & 1.5062880000 \\ \mathrm{C} & -0.6443560000 & 2.8227030000 & -1.4417320000 \\ \mathrm{C} & 2.2328000000 & 1.8462080000 & -1.4623720000 \\ \mathrm{C} & -4.3971580000 & -2.5881490000 & -1.6020840000 \\ \mathrm{C} & -2.1081330000 & -1.9263810000 & -2.0984680000 \\ \mathrm{C} & 1.3360050000 & -0.4477980000 & -1.5457370000 \\ \mathrm{C} & 1.0619820000 & 0.9220700000 & -1.5621030000 \\ \mathrm{C} & -0.2685500000 & 1.3729900000 & -1.6297030000 \\ \mathrm{C} & -1.2729650000 & 0.4147530000 & -1.7921840000 \\ \mathrm{C} & -0.9938020000 & -0.9521520000 & -1.8415120000 \\ \mathrm{C} & 0.3289790000 & -1.4039340000 & -1.6698390000\end{array}$




$\begin{array}{lrrr}\mathrm{C} & 0.6618660000 & -2.8748680000 & -1.5625380000 \\ \mathrm{C} & 0.6731650000 & -3.0119380000 & 1.3808190000 \\ \mathrm{C} & -2.8020620000 & 0.5357190000 & 1.5790940000 \\ \mathrm{C} & 0.9849700000 & 0.7618500000 & 1.8079390000 \\ \mathrm{C} & 1.3323540000 & -0.5885130000 & 1.8091170000 \\ \mathrm{C} & 0.3379450000 & -1.5602440000 & 1.5993470000 \\ \mathrm{C} & -0.9885800000 & -1.1365020000 & 1.5289590000 \\ \mathrm{C} & -1.3428560000 & 0.2136320000 & 1.6103880000 \\ \mathrm{C} & -0.3380330000 & 1.1940790000 & 1.6700350000 \\ \mathrm{H} & 5.6745280000 & 0.0871130000 & 0.8699910000 \\ \mathrm{H} & 5.2933350000 & -1.6168700000 & 1.2451790000 \\ \mathrm{H} & 5.4314230000 & -0.4395230000 & 2.5562070000 \\ \mathrm{H} & 3.4156590000 & 0.4534440000 & 0.7036490000 \\ \mathrm{H} & -5.1869050000 & 1.3167210000 & 1.8424150000 \\ \mathrm{H} & -4.6853770000 & 2.9057690000 & 2.4618740000 \\ \mathrm{H} & -5.0628090000 & 1.5804080000 & 3.5973190000 \\ \mathrm{H} & -2.5984480000 & 1.6635760000 & 3.2628330000 \\ \mathrm{H} & 3.0572850000 & 4.7343000000 & -2.7077620000 \\ \mathrm{H} & 4.1620880000 & 3.4571140000 & -3.2812930000 \\ \mathrm{H} & 3.9483270000 & 3.7243540000 & -1.5403490000 \\ \mathrm{H} & -5.1782870000 & -2.2867320000 & -0.9031580000 \\ \mathrm{H} & -4.1427820000 & -3.6396140000 & -1.4349260000 \\ \mathrm{H} & -4.7706620000 & -2.4974890000 & -2.6267060000 \\ \mathrm{H} & 1.6878420000 & 2.7415160000 & -3.2082140000 \\ \mathrm{H} & -3.2898450000 & -1.0293020000 & -0.6508910000 \\ \mathrm{H} & -0.2329090000 & -3.6098160000 & 1.2607250000 \\ \mathrm{H} & 1.2701220000 & -3.4011120000 & 2.2064630000 \\ \mathrm{H} & 0.3668120000 & 3.1950710000 & 1.3687040000 \\ \mathrm{H} & -1.0796470000 & 3.1344140000 & 2.3687950000 \\ \mathrm{H} & 1.2271980000 & -3.2101910000 & -2.4361450000 \\ \mathrm{H} & -0.2354850000 & -3.4884140000 & -1.5178890000 \\ \mathrm{H} & 0.2278150000 & 3.4729010000 & -1.3769330000 \\ \mathrm{H} & -1.2774470000 & 3.1808260000 & -2.2586430000 \\ \mathrm{H} & 1.7739940000 & 1.5031820000 & 1.8814100000 \\ \mathrm{H} & -1.7766100000 & -1.8687800000 & 1.3810070000 \\ \mathrm{H} & -2.3064910000 & 0.7429200000 & -1.8418310000 \\ \mathrm{H} & 2.3588400000 & -0.7764150000 & -1.3915530000\end{array}$




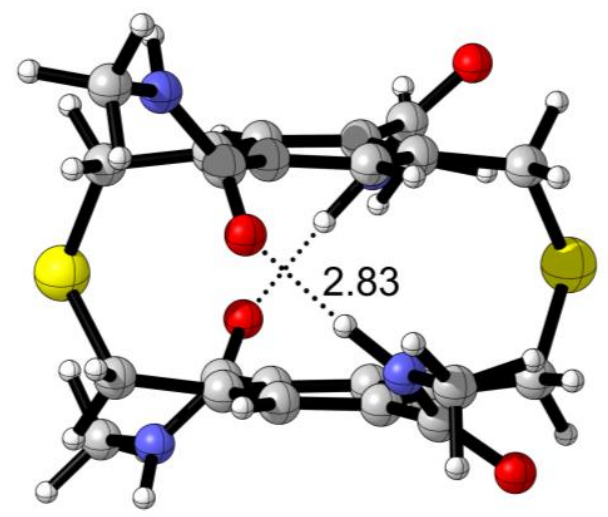

$\left(S_{\mathrm{p}}\right)-1$ boat anti-

Energy: -2246.86889133

$\begin{array}{lrrr}\text { S } & 1.0142290000 & -3.6060340000 & 0.1671040000 \\ \text { S } & 0.7623320000 & 3.4885650000 & -0.1058270000 \\ \mathrm{O} & -2.5542110000 & -2.7531070000 & -2.6670220000 \\ \mathrm{O} & 3.3720110000 & 0.9150300000 & -0.7079900000 \\ \mathrm{O} & -3.5757020000 & 0.9788060000 & 0.4627220000 \\ \mathrm{O} & 2.3874600000 & -2.2511020000 & 3.0045490000 \\ \mathrm{~N} & -3.1223690000 & 2.1572430000 & 2.3587210000 \\ \mathrm{~N} & -3.5648360000 & -1.1904570000 & -1.3471080000 \\ \mathrm{~N} & 2.7559190000 & 2.1465780000 & -2.5093710000 \\ \mathrm{~N} & 3.4035600000 & -0.8904810000 & 1.4709740000 \\ \mathrm{C} & -4.4143810000 & 2.8283180000 & 2.3949890000 \\ \mathrm{C} & -4.8686690000 & -1.8172840000 & -1.4927320000 \\ \mathrm{C} & 3.9889860000 & 2.9209790000 & -2.4931600000 \\ \mathrm{C} & 4.7057070000 & -1.4998930000 & 1.6828720000 \\ \mathrm{C} & -2.4852530000 & -1.7078020000 & -1.9771200000 \\ \mathrm{C} & 2.5044640000 & 1.2103340000 & -1.5657460000 \\ \mathrm{C} & -2.7765350000 & 1.2777250000 & 1.3866230000 \\ \mathrm{C} & 2.3276680000 & -1.3368320000 & 2.1482960000 \\ \mathrm{C} & 0.1049370000 & -3.1241430000 & -1.4178770000 \\ \mathrm{C} & -0.1146260000 & -2.9181090000 & 1.5218940000 \\ \mathrm{C} & -1.4202740000 & 0.6634940000 & 1.5076530000 \\ \mathrm{C} & -1.3490470000 & -0.7356250000 & 1.4788160000 \\ \mathrm{C} & -0.1435460000 & -1.4146830000 & 1.6270590000 \\ \mathrm{C} & 1.0243090000 & -0.6545190000 & 1.8422120000 \\ \mathrm{C} & 0.9631820000 & 0.7353860000 & 1.7987190000 \\ \mathrm{C} & -0.2430120000 & 1.4214490000 & 1.6046860000 \\ \mathrm{C} & -0.2201900000 & 2.9171560000 & 1.4075550000 \\ \mathrm{C} & -0.1938270000 & 2.7048360000 & -1.5370720000 \\ \mathrm{C} & -1.1952830000 & -0.9570860000 & -1.8105440000 \\ \mathrm{C} & 0.0237800000 & -1.6331490000 & -1.6373010000 \\ \mathrm{C} & 1.1939040000 & -0.8705100000 & -1.5807100000 \\ \mathrm{C} & 1.1801510000 & 0.5211450000 & -1.6385540000 \\ \mathrm{C} & -0.0494590000 & 1.2060520000 & -1.6898250000 \\ \mathrm{C} & -1.2104770000 & 0.4427400000 & -1.8121500000\end{array}$




$\begin{array}{rrrr}\mathrm{H} & -3.4845840000 & -0.3835190000 & -0.7285280000 \\ \mathrm{H} & -2.4865500000 & 2.2876880000 & 3.1298110000 \\ \mathrm{H} & 3.3033490000 & -0.2157410000 & 0.7110630000 \\ \mathrm{H} & 2.0986900000 & 2.2764480000 & -3.2601300000 \\ \mathrm{H} & -4.2796110000 & 3.8993050000 & 2.5650270000 \\ \mathrm{H} & -5.0517560000 & 2.4155270000 & 3.1826800000 \\ \mathrm{H} & -4.9017240000 & 2.6714310000 & 1.4327540000 \\ \mathrm{H} & 5.0425520000 & -1.3542670000 & 2.7135610000 \\ \mathrm{H} & 5.4182340000 & -1.0372350000 & 0.9989000000 \\ \mathrm{H} & 4.6621920000 & -2.5776540000 & 1.4947700000 \\ \mathrm{H} & -5.5973410000 & -1.2298940000 & -0.9325580000 \\ \mathrm{H} & -4.8569680000 & -2.8437300000 & -1.1120930000 \\ \mathrm{H} & -5.1638050000 & -1.8596350000 & -2.5452630000 \\ \mathrm{H} & 4.1136450000 & 3.3958530000 & -1.5167840000 \\ \mathrm{H} & 3.9354440000 & 3.6888250000 & -3.2655590000 \\ \mathrm{H} & 4.8573200000 & 2.2813990000 & -2.6754680000 \\ \mathrm{H} & -1.2200150000 & 3.3426050000 & 1.3244230000 \\ \mathrm{H} & 0.3007170000 & 3.4218420000 & 2.2263110000 \\ \mathrm{H} & -1.1185570000 & -3.3175910000 & 1.3608080000 \\ \mathrm{H} & 0.3157650000 & -3.3542180000 & 2.4248220000 \\ \mathrm{H} & -1.2488670000 & 2.9616060000 & -1.4117220000 \\ \mathrm{H} & 0.1753790000 & 3.2634730000 & -2.4010640000 \\ \mathrm{H} & -0.8762820000 & -3.5919880000 & -1.4299170000 \\ \mathrm{H} & 0.7017320000 & -3.6036930000 & -2.1983700000 \\ \mathrm{H} & -2.2627680000 & -1.2986700000 & 1.3140580000 \\ \mathrm{H} & 1.8834940000 & 1.3051780000 & 1.8809700000 \\ \mathrm{H} & 2.1438910000 & -1.3741460000 & -1.4335160000 \\ \mathrm{H} & -2.1659730000 & 0.9532470000 & -1.8714970000\end{array}$

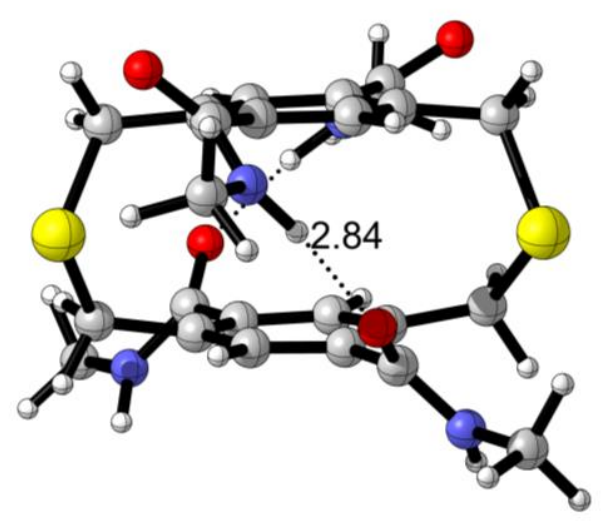

$\left(S_{\mathrm{p}}\right)-1$ boat syn-

Energy: -2246.86835434

$\begin{array}{lrrr}\mathrm{S} & 1.6105420000 & -3.3000170000 & 0.0886590000 \\ \mathrm{~S} & 0.1297450000 & 3.6502150000 & -0.1351320000 \\ \mathrm{O} & 2.2496640000 & 2.8549110000 & -2.6241230000 \\ \mathrm{O} & -3.8507090000 & 0.3466820000 & 0.4793350000 \\ \mathrm{O} & 3.3379100000 & -0.1975450000 & 1.1520820000 \\ \mathrm{O} & -1.8776240000 & -3.1186150000 & -2.5800890000\end{array}$




\begin{tabular}{|c|c|c|c|}
\hline $\mathrm{N}$ & -3.1702600000 & -1.7100870000 & -1.3522040000 \\
\hline & -3.5900910000 & 1.6786230000 & 2.2981540000 \\
\hline & 2.7390080000 & -1.6736730000 & 2.7664950000 \\
\hline & 3.3258030000 & 1.6640550000 & -0.9961510000 \\
\hline C & -4.3284250000 & -2.5795310000 & -1.4848720000 \\
\hline C & -4.9856590000 & 2.0998240000 & 2.2879690000 \\
\hline $\mathrm{C}$ & -3.1022120000 & 0.8262940000 & 1.3680970000 \\
\hline $\mathrm{C}$ & 4.1018770000 & -2.1586430000 & 2.9344990000 \\
\hline C & 2.4361380000 & -0.7603200000 & 1.8169810000 \\
\hline$C$ & -2.0012490000 & -2.0375710000 & -1.9520620000 \\
\hline $\mathrm{C}$ & 0.2114320000 & -2.8452860000 & 1.2695800000 \\
\hline $\mathrm{C}$ & 0.8656960000 & -2.9045550000 & -1.6127420000 \\
\hline $\mathrm{C}$ & 4.4561930000 & 2.5763370000 & -0.9752310000 \\
\hline $\mathrm{C}$ & 2.2888380000 & 1.8832650000 & -1.8315330000 \\
\hline $\mathrm{C}$ & 1.1750340000 & 0.8785 & 790000 \\
\hline $\mathrm{C}$ & 1.4649760000 & -0.4821540000 & -1.7546680000 \\
\hline $\mathrm{C}$ & 0.4635590000 & -1.4612380000 & -1.7624360000 \\
\hline $\mathrm{C}$ & -0.8773090000 & -1.0467280000 & -1.8370670000 \\
\hline $\mathrm{C}$ & -1.1653170000 & 0.3266220000 & 40000 \\
\hline $\mathrm{C}$ & 900000 & 1.2976 & 10000 \\
\hline $\mathrm{C}$ & -0.5452960000 & 2.7531040000 & -1.6651360000 \\
\hline $\mathrm{C}$ & -0.8827370000 & 2.8919560000 & 1.2633800000 \\
\hline $\mathrm{C}$ & -1.3435340000 & -0.8998850000 & 380000 \\
\hline $\mathrm{C}$ & -0.0305770000 & -1.3645700000 & 10000 \\
\hline $\mathrm{C}$ & 470000 & -0.4062 & 90000 \\
\hline $\mathrm{C}$ & 0.6838740000 & 0.9526450000 & 390000 \\
\hline $\mathrm{C}$ & -0.6280300000 & 1.4190490000 & 610000 \\
\hline $\mathrm{C}$ & -1.6560570000 & 0.4630210000 & 140000 \\
\hline $\mathrm{H}$ & 4.4606170000 & -2.5948650000 & 0540000 \\
\hline $\mathrm{H}$ & 4.1127790000 & -2.9189300000 & 3.7158810000 \\
\hline $\mathrm{H}$ & 4.7727070000 & -1.3415620000 & 3.2121400000 \\
\hline $\mathrm{H}$ & 2.0065720000 & -2.0490690000 & 3.3447640000 \\
\hline $\mathrm{H}$ & -5.1145180000 & 2.9146670000 & 3.0007980000 \\
\hline $\mathrm{H}$ & -5.6487700000 & 1.2727340000 & 2.5579200000 \\
\hline $\mathrm{H}$ & -5.2629470000 & 2.4432920000 & 1.2890620000 \\
\hline $\mathrm{H}$ & -2.9869710000 & 1.9859500000 & 3.0436530000 \\
\hline $\mathrm{H}$ & -5.1702360000 & -2.1077770000 & -0.9762560000 \\
\hline $\mathrm{H}$ & -4.1385870000 & -3.5633060000 & -1.0431090000 \\
\hline $\mathrm{H}$ & -4.5763630000 & -2.7354780000 & -2.5386720000 \\
\hline $\mathrm{H}$ & 5.1769240000 & 2.2172310000 & -0.2393790000 \\
\hline $\mathrm{H}$ & 4.1356170000 & 3.5897120000 & -0.7102950000 \\
\hline $\mathrm{H}$ & 4.9328820000 & 2.6257830000 & -1.9583940000 \\
\hline $\mathrm{H}$ & -3.2767590000 & -0.8787070000 & -0.7741700000 \\
\hline $\mathrm{H}$ & 3.2769520000 & 0.9358260000 & -0.2838090000 \\
\hline $\mathrm{H}$ & -1.9351820000 & 3.0958200000 & 1.0657930000 \\
\hline 14 & -0.5811520000 & 3.4889480000 & 2.1299270000 \\
\hline $\mathrm{H}$ & -0.7030420000 & -3.3107850000 & 0.8930610000 \\
\hline
\end{tabular}




$\begin{array}{rrrr}\mathrm{H} & 0.4833470000 & -3.3689680000 & 2.1905970000 \\ \mathrm{H} & -1.6300790000 & 2.8826960000 & -1.6807180000 \\ \mathrm{H} & -0.0905110000 & 3.3228720000 & -2.4768920000 \\ \mathrm{H} & 0.0387710000 & -3.5829020000 & -1.8044940000 \\ \mathrm{H} & 1.6865660000 & -3.1570380000 & -2.2885130000 \\ \mathrm{H} & -2.1523310000 & -1.6106170000 & 1.2955960000 \\ \mathrm{H} & 1.5016630000 & 1.6662190000 & 1.6207730000 \\ \mathrm{H} & 2.5023050000 & -0.7991910000 & -1.7098640000 \\ \mathrm{H} & -2.2018190000 & 0.6488220000 & -1.8446340000\end{array}$

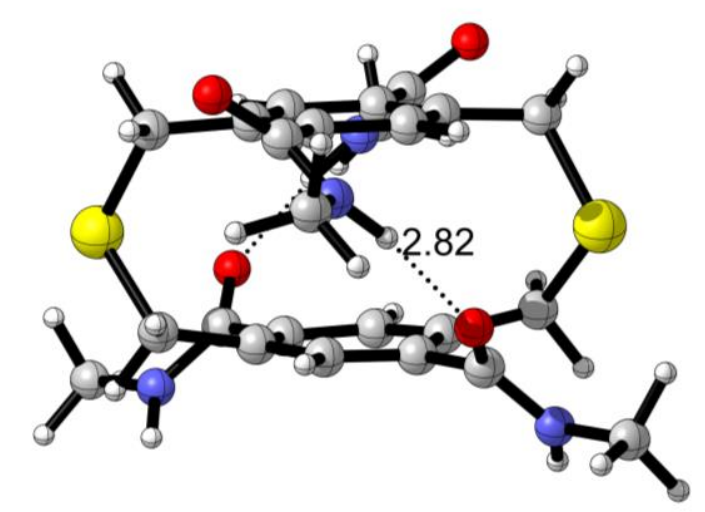

$\left(S_{\mathrm{p}}\right)$-1 chair syn-

Energy:-2246.86917972

$\begin{array}{lrrr}\mathrm{S} & -2.4174990000 & 2.8051340000 & -0.1336040000 \\ \mathrm{~S} & 2.4174890000 & -2.8050940000 & -0.1336020000 \\ \mathrm{O} & 3.5779590000 & 0.5625730000 & 0.8771860000 \\ \mathrm{O} & -1.2945600000 & -3.4329230000 & -2.5230960000 \\ \mathrm{O} & 1.2942380000 & 3.4327410000 & -2.5232580000 \\ \mathrm{O} & -3.5777820000 & -0.5625980000 & 0.8775250000 \\ \mathrm{~N} & -3.4656450000 & 1.0143550000 & 2.5045860000 \\ \mathrm{~N} & 2.7262390000 & 2.4049810000 & -1.0808310000 \\ \mathrm{~N} & -2.7263810000 & -2.4052050000 & -1.0804640000 \\ \mathrm{~N} & 3.4659140000 & -1.0140550000 & 2.5045760000 \\ \mathrm{C} & -4.9114710000 & 1.1886820000 & 2.5384740000 \\ \mathrm{C} & 3.6455170000 & 3.5314390000 & -1.0954790000 \\ \mathrm{C} & -3.6456510000 & -3.5316680000 & -1.0950130000 \\ \mathrm{C} & -1.6045360000 & -2.4284240000 & -1.8352630000 \\ \mathrm{C} & 1.6043070000 & 2.4282190000 & -1.8355010000 \\ \mathrm{C} & -2.8846090000 & 0.1846350000 & 1.6113320000 \\ \mathrm{C} & -1.3946460000 & 2.5689680000 & -1.7141170000 \\ \mathrm{C} & -1.1145960000 & 2.6903620000 & 1.2268060000 \\ \mathrm{C} & 4.9117310000 & -1.1885060000 & 2.5382720000 \\ \mathrm{C} & 2.8848210000 & -0.1843750000 & 1.6113210000 \\ \mathrm{C} & 1.3895230000 & -0.1484250000 & 1.5886840000 \\ \mathrm{C} & 0.7994590000 & 1.1160150000 & 1.5467680000 \\ \mathrm{C} & -0.5836170000 & 1.2978330000 & 1.4973320000 \\ \mathrm{C} & -1.3893120000 & 0.1486490000 & 1.5886680000 \\ \mathrm{C} & -0.7992510000 & -1.1157970000 & 1.5468740000\end{array}$




$\begin{array}{lrrr}\mathrm{C} & 0.5838270000 & -1.2976200000 & 1.4974450000 \\ \mathrm{C} & 1.1147860000 & -2.6901720000 & 1.2269920000 \\ \mathrm{C} & 1.3944230000 & -2.5691420000 & -1.7139990000 \\ \mathrm{C} & -1.3741410000 & 0.0639890000 & -1.7969010000 \\ \mathrm{C} & -0.6514150000 & 1.2591090000 & -1.7862670000 \\ \mathrm{C} & 0.7547580000 & 1.1890710000 & -1.8003940000 \\ \mathrm{C} & 1.3739030000 & -0.0641810000 & -1.7970060000 \\ \mathrm{C} & 0.6511790000 & -1.2592960000 & -1.7862270000 \\ \mathrm{C} & -0.7549950000 & -1.1892650000 & -1.8002590000 \\ \mathrm{H} & -4.0834730000 & -3.6694260000 & -2.0885300000 \\ \mathrm{H} & -4.4388530000 & -3.3339510000 & -0.3728460000 \\ \mathrm{H} & -3.1299590000 & -4.4601210000 & -0.8308780000 \\ \mathrm{H} & -2.9488980000 & -1.6260860000 & -0.4600560000 \\ \mathrm{H} & 4.0830680000 & 3.6693150000 & -2.0890970000 \\ \mathrm{H} & 4.4389110000 & 3.3336180000 & -0.3735510000 \\ \mathrm{H} & 3.1299140000 & 4.4598690000 & -0.8310840000 \\ \mathrm{H} & 2.9488060000 & 1.6258580000 & -0.4604450000 \\ \mathrm{H} & -5.2650270000 & 1.5814750000 & 1.5808640000 \\ \mathrm{H} & -5.1651440000 & 1.8897750000 & 3.3339310000 \\ \mathrm{H} & -5.4085840000 & 0.2330490000 & 2.7220860000 \\ \mathrm{H} & 5.2651310000 & -1.5812340000 & 1.5805780000 \\ \mathrm{H} & 5.1654370000 & -1.8897050000 & 3.3336250000 \\ \mathrm{H} & 5.4089560000 & -0.2329380000 & 2.7219210000 \\ \mathrm{H} & -2.8895670000 & 1.5409190000 & 3.1391640000 \\ \mathrm{H} & 2.8898840000 & -1.5405030000 & 3.1392940000 \\ \mathrm{H} & 0.7269540000 & -3.4190070000 & -1.8328970000 \\ \mathrm{H} & 2.1599930000 & -2.6256420000 & -2.4920060000 \\ \mathrm{H} & -2.1603180000 & 2.6253680000 & -2.4920300000 \\ \mathrm{H} & -0.7271910000 & 3.4188160000 & -1.8332070000 \\ \mathrm{H} & 0.2882450000 & -3.3512270000 & 0.9545970000 \\ \mathrm{H} & 1.6139620000 & -3.1362670000 & 2.0914680000 \\ \mathrm{H} & -1.6136410000 & 3.1365430000 & 2.0913130000 \\ \mathrm{H} & -0.2880890000 & 3.3513820000 & 0.9542180000 \\ \mathrm{H} & -2.4583500000 & 0.1184660000 & -1.7815150000 \\ \mathrm{H} & 2.4581130000 & -0.1186580000 & -1.7816530000 \\ \mathrm{H} & 1.4523710000 & 1.9810760000 & 1.4873500000 \\ \mathrm{H} & -1.4521630000 & -1.9808630000 & 1.4875230000\end{array}$




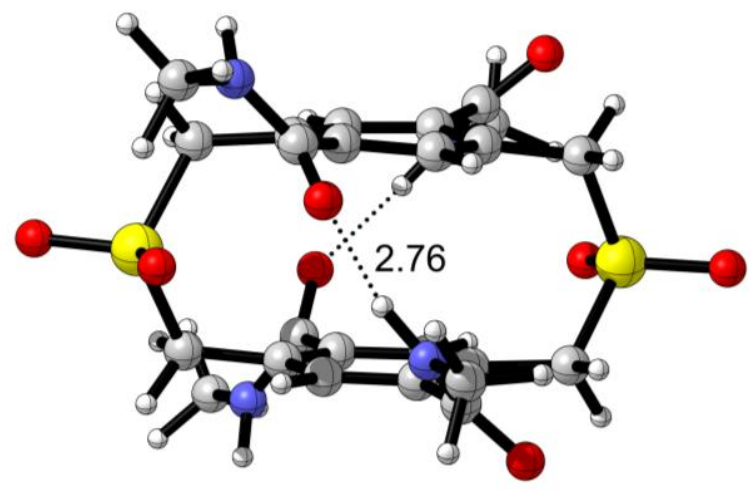

$\left(S_{\mathrm{p}}\right)$-2 chair anti-

Energy: -2547.20401869

$\begin{array}{lrrr}\text { S } & -3.0261700000 & 1.9904500000 & -0.0483750000 \\ \mathrm{~S} & 3.1636820000 & -2.0377720000 & -0.1109660000 \\ \mathrm{O} & -3.8262870000 & 3.4064850000 & 0.0531920000 \\ \mathrm{O} & -3.8641310000 & 0.5991930000 & -0.2129280000 \\ \mathrm{O} & 4.0730170000 & -3.3870310000 & -0.1480440000 \\ \mathrm{O} & 3.8841650000 & -0.5704280000 & -0.1791070000 \\ \mathrm{O} & 1.6655210000 & 3.1063200000 & -0.4914130000 \\ \mathrm{O} & -3.0330870000 & -2.0436500000 & 0.8757620000 \\ \mathrm{O} & -0.2027600000 & -3.4878010000 & -2.8829550000 \\ \mathrm{O} & 3.4809280000 & 0.0177590000 & 2.9594190000 \\ \mathrm{~N} & 3.2071010000 & 1.8324910000 & 1.5884990000 \\ \mathrm{~N} & -1.9028210000 & -3.1483240000 & -1.3904370000 \\ \mathrm{~N} & 0.7402130000 & 3.5645510000 & -2.5178550000 \\ \mathrm{~N} & -3.5366180000 & -0.6311600000 & 2.5798150000 \\ \mathrm{C} & 4.5784080000 & 2.2776240000 & 1.7842370000 \\ \mathrm{C} & -2.4467860000 & -4.4843940000 & -1.5848730000 \\ \mathrm{C} & 1.2136140000 & 4.9446670000 & -2.5273260000 \\ \mathrm{C} & 1.0087000000 & 2.7255830000 & -1.4896570000 \\ \mathrm{C} & -0.8214350000 & -2.7452680000 & -2.0805250000 \\ \mathrm{C} & 2.7912030000 & 0.6862370000 & 2.1550140000 \\ \mathrm{C} & -4.9489740000 & -0.9935060000 & 2.5666630000 \\ \mathrm{C} & -2.6914990000 & -1.1164370000 & 1.6462360000 \\ \mathrm{C} & -1.9167740000 & 2.1256650000 & -1.5892570000 \\ \mathrm{C} & -1.9935570000 & 1.9220640000 & 1.5621390000 \\ \mathrm{C} & 1.3884160000 & 0.2480310000 & 1.8261430000 \\ \mathrm{C} & 0.3573980000 & 1.1857660000 & 1.7895440000 \\ \mathrm{C} & -0.9882400000 & 0.8167780000 & 1.6692160000 \\ \mathrm{C} & -1.3014130000 & -0.5561940000 & 1.6314720000 \\ \mathrm{C} & -0.2619980000 & -1.4855430000 & 1.5466180000 \\ \mathrm{C} & 1.0796150000 & -1.1106370000 & 1.6292680000 \\ \mathrm{C} & 2.1430200000 & -2.1522230000 & 1.5022100000 \\ \mathrm{C} & 2.0316800000 & -2.1403960000 & -1.6333590000 \\ \mathrm{C} & 0.4837830000 & 1.3286460000 & -1.6194910000 \\ \mathrm{C} & -0.8903430000 & 1.0434710000 & -1.6986190000\end{array}$




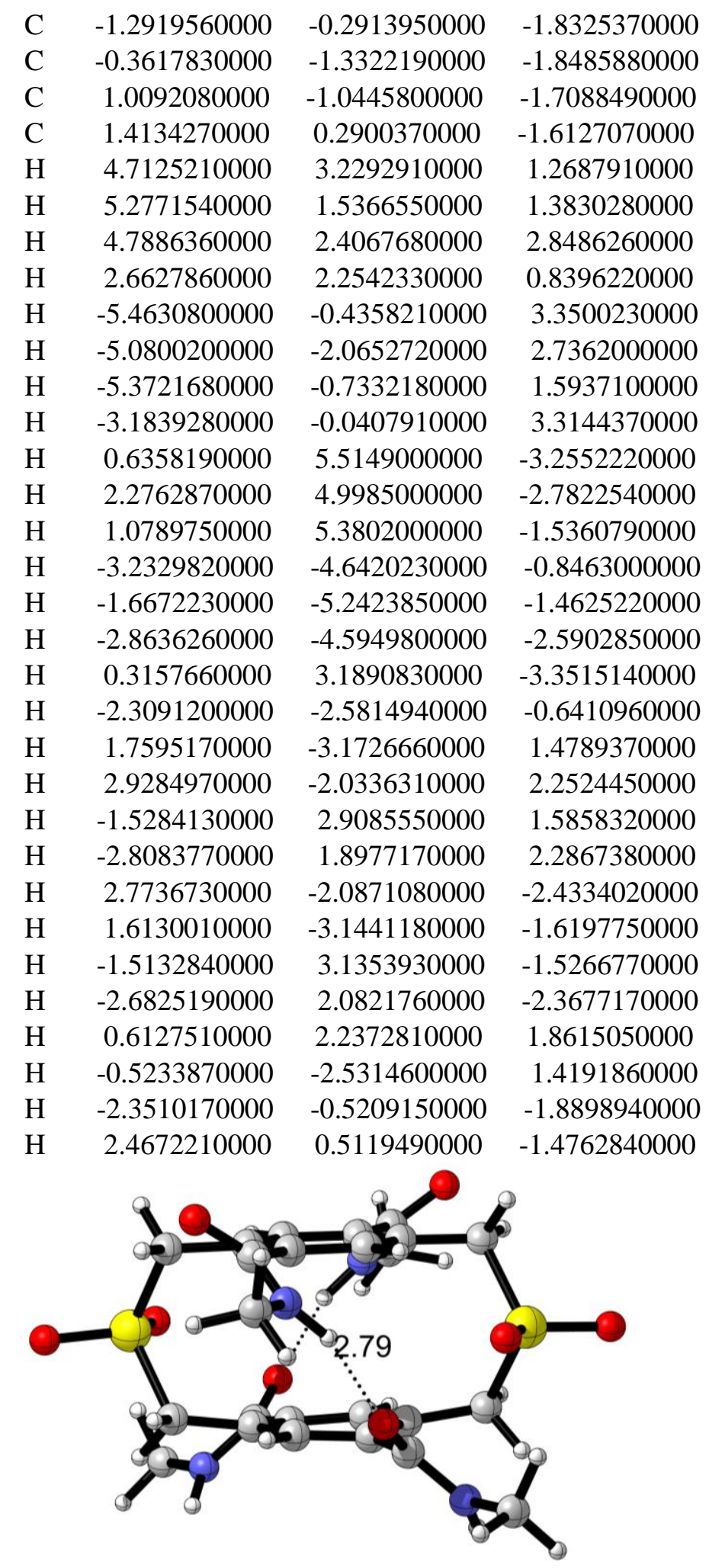

$\left(S_{\mathrm{p}}\right)$-2 chair syn-

Energy: -2547.20491395

$\begin{array}{llll}\text { S } & -3.2329440000 & 1.7864790000 & -0.2252730000\end{array}$ 


\begin{tabular}{|c|c|c|c|}
\hline & 329800000 & -1.7863930000 & 251440000 \\
\hline 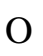 & -4.2088530000 & 3.0874310000 & -0.1450840000 \\
\hline & -3.8922410000 & 0.2904620000 & 70000 \\
\hline & 4.2089260000 & -3.0873230000 & -0.1451170000 \\
\hline & 3.8922160000 & -0.2903470000 & 10000 \\
\hline & -2.9533860000 & -2.1491 & 10000 \\
\hline 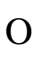 & -0.0334870000 & 70000 & -2.5691470000 \\
\hline & 0.0336040000 & 70000 & 10000 \\
\hline & 2.9534730000 & 0000 & 20000 \\
\hline & 3.5391920000 & 0.57 & 0000 \\
\hline & -1.6742720000 & -3.24 & -1.1 \\
\hline & 710000 & 3.24 & 000 \\
\hline & -3.5394460000 & -0.57 & 60000 \\
\hline & 390000 & 1.014 & 30000 \\
\hline & -2.1122450000 & -4.63 & 20000 \\
\hline & 480000 & 4.63 & 000 \\
\hline ( & 0.63 & 2.8 & 000 \\
\hline C & -0.6328750000 & -2.84 & 0000 \\
\hline & 50000 & 1.10 & 0000 \\
\hline $\mathrm{C}$ & 2.1859170000 & -1.91 & 0000 \\
\hline & 2.1430380000 & -1.96 & 00 \\
\hline C & -4.9 & -1.0 & 000 \\
\hline C & -2.6784130000 & 0000 & 60000 \\
\hline C & -1.32 & -0.4 & 0000 \\
\hline & 30000 & -1.3 & 000 \\
\hline $\mathrm{C}$ & 00000 & -0.91 & 000 \\
\hline $\mathrm{C}$ & & 0.4 & \\
\hline $\mathrm{C}$ & 900000 & 000 & 0000 \\
\hline $\mathrm{C}$ & 370000 & 00 & 000 \\
\hline & 80000 & 000 & 000 \\
\hline $\mathrm{C}$ & 560000 & 00 & 0000 \\
\hline & 070000 & 0.43 & 0000 \\
\hline 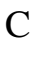 & 1.0458000000 & -0.94 & 0000 \\
\hline $\mathrm{C}$ & -0.2 & -1.3 & 0000 \\
\hline & -1.31 & -0.42 & 0000 \\
\hline $\mathrm{C}$ & 770000 & 00 & 000 \\
\hline 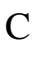 & 0.2920140000 & 000 & 0000 \\
\hline . & 0000 & 000 & 0000 \\
\hline $\mathrm{H}$ & 230000 & 000 & 0000 \\
\hline $\mathrm{H}$ & 530000 & 0000 & 0000 \\
\hline $\mathrm{H}$ & 660000 & 2.63 & 00000 \\
\hline $\mathrm{H}$ & 6340000 & -4.89 & 370000 \\
\hline H & 500000 & -4.75 & 9720000 \\
\hline$\Pi$ & -1.2788920000 & -5.30 & -0.9209100000 \\
\hline $\mathrm{H}$ & -2.1422850000 & -2.63 & 460000 \\
\hline $\mathrm{H}$ & 5.3826970000 & 0.8558220000 & 1.7059750000 \\
\hline $\mathrm{H}$ & 5.4615300000 & 0.4280500000 & 3.4369380000 \\
\hline
\end{tabular}




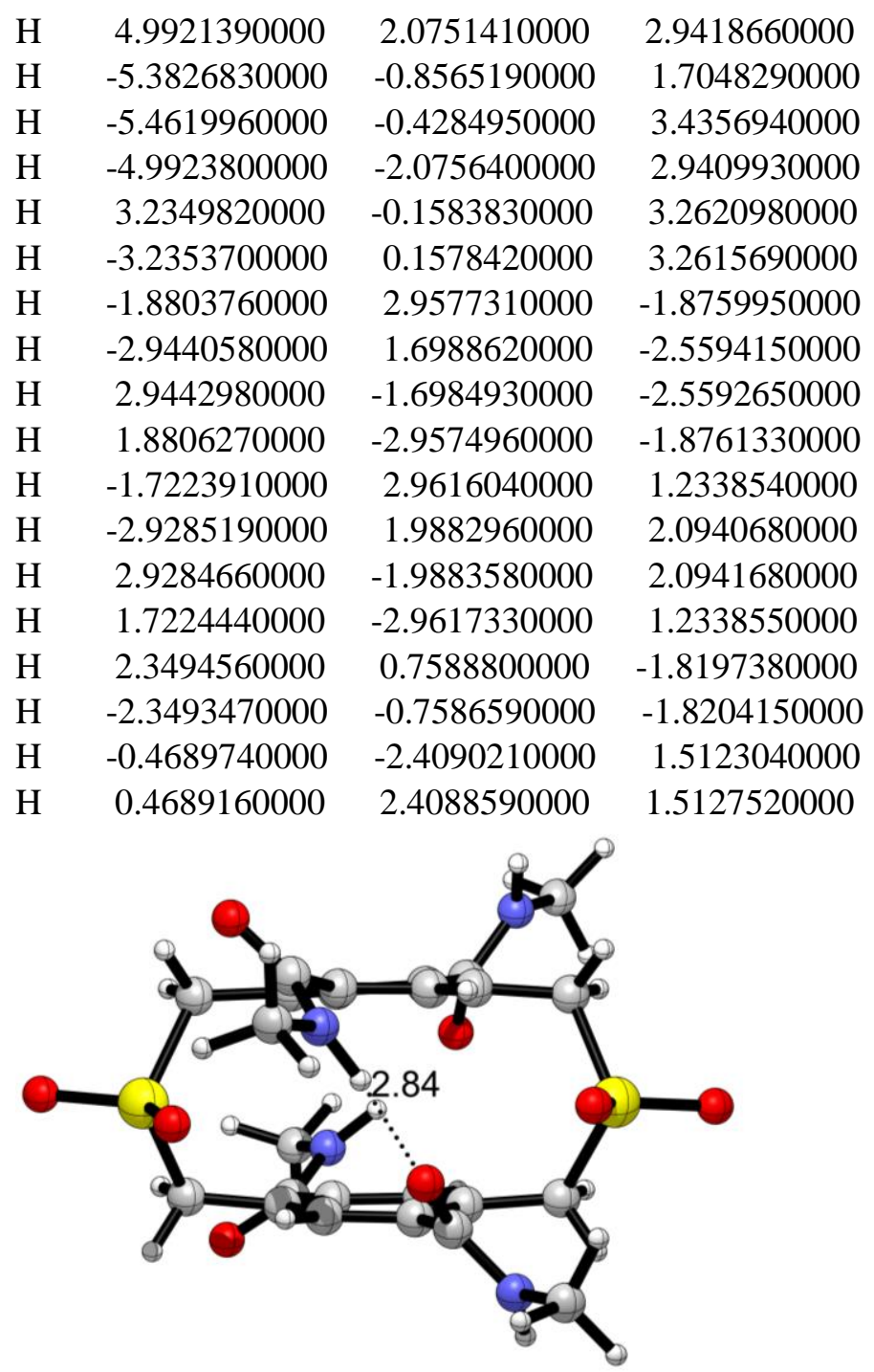

$\left(S_{\mathrm{p}}\right)$-2 boat anti-

Energy: -2547.20199977

$\begin{array}{lrrr}\mathrm{S} & 3.4816220000 & -1.3554840000 & 0.0416750000 \\ \mathrm{~S} & -2.7925570000 & 2.2582160000 & -0.0409420000 \\ \mathrm{O} & 3.8366370000 & 0.2371900000 & -0.0446170000 \\ \mathrm{O} & -1.6840250000 & 3.4383040000 & 0.1524760000 \\ \mathrm{O} & 3.0539600000 & 0.7716450000 & 3.0775090000 \\ \mathrm{O} & -2.8223900000 & -2.5791480000 & 0.4822310000 \\ \mathrm{O} & 1.1304420000 & 3.1824930000 & -0.8087930000 \\ \mathrm{O} & 4.6806140000 & -2.4513240000 & 0.1516480000 \\ \mathrm{O} & 0.7753060000 & -3.7375870000 & -2.6910630000 \\ \mathrm{O} & -4.3681370000 & 2.6476290000 & -0.1942180000 \\ \mathrm{~N} & -1.0931730000 & -3.7415230000 & -1.3819780000 \\ \mathrm{~N} & -0.2787000000 & 3.4066700000 & -2.5796990000 \\ \mathrm{~N} & -3.4442860000 & -1.7102440000 & 2.4838040000 \\ \mathrm{~N} & 2.4686870000 & 2.4085600000 & 1.5787320000 \\ \mathrm{C} & -1.2885760000 & -5.1739470000 & -1.5523430000\end{array}$




\begin{tabular}{|c|c|c|c|}
\hline $\mathrm{C}$ & -0.2708070000 & 4.8630120000 & -2.5084010000 \\
\hline $\mathrm{C}$ & -4.6854210000 & -2.4771060000 & 2.5241010000 \\
\hline - & 3.6885530000 & 3.1675240000 & 1.8101480000 \\
\hline & 2.3844240000 & -1.6752940000 & 1.5759850000 \\
\hline $\mathrm{C}$ & 2.2878620000 & 1.2332680000 & 2.1973220000 \\
\hline $\mathrm{C}$ & -2.5821080000 & -1.8111380000 & 1.4474740000 \\
\hline $\mathrm{C}$ & -0.1873460000 & 1.1251620000 & 1.7993360000 \\
\hline $\mathrm{C}$ & 1.0430610000 & 0.4674300000 & 1.8299220000 \\
\hline $\mathrm{C}$ & 1.0914160000 & -0.9251130000 & 1.6195650000 \\
\hline $\mathrm{C}$ & -0.1080860000 & -1.6250640000 & 130000 \\
\hline $\mathrm{C}$ & -1.3431120000 & -0.9773860000 & 1.5299900000 \\
\hline $\mathrm{C}$ & -1.3886300000 & 0.4235260000 & 1.6375000000 \\
\hline C & -2.6773520000 & 1.1722870000 & 1.5194990000 \\
\hline $\mathrm{C}$ & 0.3608300000 & 2.6659950000 & -1.6478800000 \\
\hline $\mathrm{C}$ & 2.5442060000 & -1.7693940000 & -1.5603000000 \\
\hline C & -0.0680600000 & -3.1182320000 & -1.9986920000 \\
\hline $\mathrm{C}$ & -2.4161240000 & 1.2605100000 & -1.6351840000 \\
\hline $\mathrm{C}$ & 1.3141750000 & 0.4043300000 & -1.6705230000 \\
\hline $\mathrm{C}$ & 1.2716920000 & -0.9921510000 & -1.7072020000 \\
\hline $\mathrm{C}$ & 0.0225350000 & -1.6277370000 & 50000 \\
\hline $\mathrm{C}$ & -1.1369380000 & -0.8511710000 & -1.8034920000 \\
\hline $\mathrm{C}$ & -1.1021250000 & 0.5463180000 & 270000 \\
\hline $\mathrm{C}$ & 0.1555260000 & 1.1802680000 & 360000 \\
\hline $\mathrm{H}$ & -1.7203390000 & -3.2401180000 & 380000 \\
\hline $\mathrm{H}$ & -3.1725530000 & -1.1748330000 & 10000 \\
\hline H & 1.9162160000 & 2.6439580000 & 00000 \\
\hline $\mathrm{H}$ & -0.7251060000 & 2.9509620000 & 70000 \\
\hline $\mathrm{H}$ & -5.3251440000 & -2.0749780000 & 3.3096870000 \\
\hline $\mathrm{H}$ & -4.4893090000 & -3.5352970000 & 2.7197430000 \\
\hline $\mathrm{H}$ & -5.1969080000 & -2.3973750000 & 1.5633000000 \\
\hline $\mathrm{H}$ & 3.8200540000 & 3.3580440000 & 2.8776540000 \\
\hline $\mathrm{H}$ & 3.6126020000 & 4.1144800000 & 1940000 \\
\hline $\mathrm{H}$ & 4.5566080000 & 2.6062050000 & 1.4496800000 \\
\hline $\mathrm{H}$ & -1.4543840000 & -5.4196940000 & -2.6050640000 \\
\hline $\mathrm{H}$ & -2.1578790000 & -5.4747220000 & -0.9667380000 \\
\hline $\mathrm{H}$ & -0.4089200000 & -5.7315410000 & -1.2167280000 \\
\hline $\mathrm{H}$ & -0.6633820000 & 5.1711220000 & -1.5366310000 \\
\hline $\mathrm{H}$ & -0.9073290000 & 5.2577520000 & -3.3010300000 \\
\hline $\mathrm{H}$ & 0.7426840000 & 5.2559800000 & -2.6242770000 \\
\hline $\mathrm{H}$ & -3.5706490000 & 0.5525640000 & 1.4617080000 \\
\hline $\mathrm{H}$ & -2.8077970000 & 1.9408220000 & 2.2853580000 \\
\hline $\mathrm{H}$ & 2.2624520000 & -2.7588340000 & 1.5619110000 \\
\hline $\mathrm{H}$ & 3.0611530000 & -1.3598270000 & 2.3745750000 \\
\hline $\mathrm{H}$ & -3.2649140000 & 0.5764080000 & -1.6791370000 \\
\hline $\mathrm{H}$ & -2.5638040000 & 2.0558330000 & -2.3672210000 \\
\hline $\mathrm{H}$ & 2.4162050000 & -2.8491120000 & -1.5579110000 \\
\hline $\mathrm{H}$ & 3.2981260000 & -1.5028020000 & -2.3041390000 \\
\hline
\end{tabular}




$\begin{array}{lrrr}\mathrm{H} & -0.0872610000 & -2.6998670000 & 1.3213160000 \\ \mathrm{H} & -0.2126330000 & 2.2066300000 & 1.8856260000 \\ \mathrm{H} & 2.2720440000 & 0.9013230000 & -1.5558300000 \\ \mathrm{H} & -2.0993880000 & -1.3500440000 & -1.8435250000\end{array}$

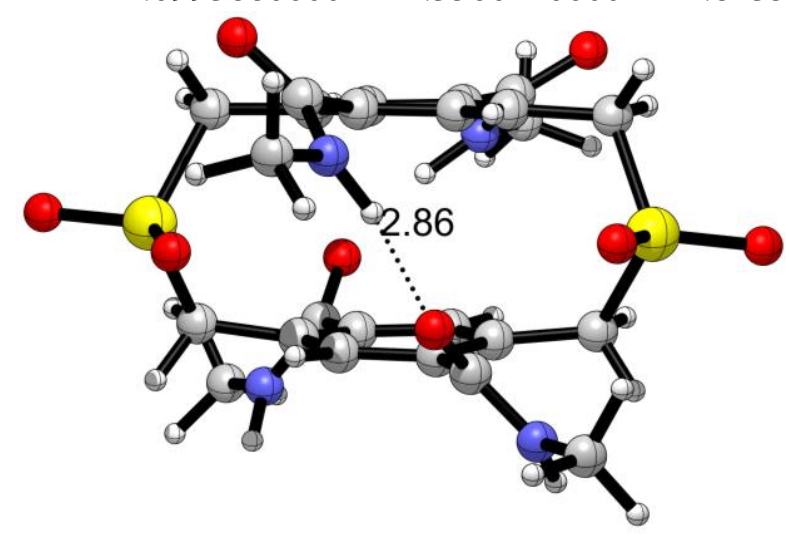

$\left(S_{\mathrm{p}}\right)$-2 boat syn-

Energy: -2547.20069965

$\begin{array}{lrrr}\mathrm{S} & 3.2378330000 & -1.7401260000 & -0.0346040000 \\ \mathrm{~S} & -2.5056690000 & 2.6403850000 & -0.1275510000 \\ \mathrm{O} & -1.2163100000 & 3.5766050000 & 0.2238450000 \\ \mathrm{O} & 3.8284290000 & -0.2202350000 & 0.0459890000 \\ \mathrm{O} & 2.4671210000 & 2.1236380000 & 1.2306840000 \\ \mathrm{O} & -3.2052950000 & -2.3213770000 & 0.5236290000 \\ \mathrm{O} & 0.3790050000 & -3.7282420000 & -2.6150820000 \\ \mathrm{O} & -0.1278950000 & 3.4038140000 & -2.8183970000 \\ \mathrm{O} & 4.2559060000 & -3.0009000000 & 0.1336280000 \\ \mathrm{O} & -3.9896540000 & 3.3001640000 & -0.2517000000 \\ \mathrm{~N} & -3.7884850000 & -1.2453290000 & 2.4348770000 \\ \mathrm{~N} & 3.1051610000 & 0.6378020000 & 2.8309510000 \\ \mathrm{~N} & 1.4843930000 & 3.2813610000 & -1.1935380000 \\ \mathrm{~N} & -1.4992580000 & -3.4892810000 & -1.3556890000 \\ \mathrm{C} & -5.1154710000 & -1.8526070000 & 2.4696460000 \\ \mathrm{C} & 4.4475220000 & 1.1989210000 & 2.9375150000 \\ \mathrm{C} & 2.2472540000 & 1.0846690000 & 1.8896140000 \\ \mathrm{C} & -2.6566850000 & 1.4613890000 & 1.3626530000 \\ \mathrm{C} & -2.9121050000 & -1.5187200000 & 1.4441170000 \\ \mathrm{C} & -0.4354070000 & -1.6169960000 & 1.4625590000 \\ \mathrm{C} & -1.5851930000 & -0.8303850000 & 1.5131830000 \\ \mathrm{C} & -1.4695600000 & 0.5697420000 & 1.5557940000 \\ \mathrm{C} & -0.1932570000 & 1.1231220000 & 1.6905560000 \\ \mathrm{C} & 0.9558510000 & 0.3303240000 & 1.7277770000 \\ \mathrm{C} & 0.8487760000 & -1.0643430000 & 1.5453370000 \\ \mathrm{C} & 2.0095960000 & -2.0052860000 & 1.4122610000 \\ \mathrm{C} & 1.7339200000 & 4.7143430000 & -1.2308200000 \\ \mathrm{C} & 0.5253390000 & 2.7513780000 & -1.9676630000 \\ \mathrm{C} & 2.3541170000 & -1.8933390000 & -1.7091830000 \\ \mathrm{C} & -1.8465980000 & -4.8945390000 & -1.5113560000\end{array}$




$\begin{array}{lrrr}\mathrm{C} & -0.4010200000 & -2.9915770000 & -1.9612540000 \\ \mathrm{C} & -2.2259250000 & 1.6702100000 & -1.7590560000 \\ \mathrm{C} & 1.3481910000 & 0.3874880000 & -1.7859080000 \\ \mathrm{C} & 1.1564770000 & -0.9991050000 & -1.7867740000 \\ \mathrm{C} & -0.1528390000 & -1.5138720000 & -1.8271590000 \\ \mathrm{C} & -1.2267600000 & -0.6186860000 & -1.7944520000 \\ \mathrm{C} & -1.0364300000 & 0.7655240000 & -1.7709050000 \\ \mathrm{C} & 0.2740090000 & 1.2759680000 & -1.8034590000 \\ \mathrm{H} & 4.9730890000 & 1.0431980000 & 1.9920800000 \\ \mathrm{H} & 4.9790100000 & 0.6919920000 & 3.7434270000 \\ \mathrm{H} & 4.3999970000 & 2.2700190000 & 3.1476550000 \\ \mathrm{H} & 2.8317220000 & -0.1074970000 & 3.4485000000 \\ \mathrm{H} & -5.7084840000 & -1.3600150000 & 3.2399840000 \\ \mathrm{H} & -5.0508320000 & -2.9225170000 & 2.6865070000 \\ \mathrm{H} & -5.6052250000 & -1.7302380000 & 1.5016080000 \\ \mathrm{H} & -3.4941920000 & -0.6664420000 & 3.2052780000 \\ \mathrm{H} & -2.7624980000 & -5.0842890000 & -0.9507550000 \\ \mathrm{H} & -1.0462920000 & -5.5412830000 & -1.1389900000 \\ \mathrm{H} & -2.0054290000 & -5.1391760000 & -2.5653100000 \\ \mathrm{H} & 2.5316110000 & 4.9431350000 & -0.5237880000 \\ \mathrm{H} & 0.8266760000 & 5.2619150000 & -0.9561760000 \\ \mathrm{H} & 2.0334000000 & 5.0251730000 & -2.2347940000 \\ \mathrm{H} & -2.0937700000 & -2.9232760000 & -0.7518700000 \\ \mathrm{H} & 1.8522980000 & 2.7600130000 & -0.3977930000 \\ \mathrm{H} & -3.5981530000 & 0.9427150000 & 1.1918230000 \\ \mathrm{H} & -2.7859350000 & 2.1988730000 & 2.1588830000 \\ \mathrm{H} & 1.6889530000 & -3.0368070000 & 1.2579510000 \\ \mathrm{H} & 2.7205970000 & -1.9844730000 & 2.2395100000 \\ \mathrm{H} & -3.1786160000 & 1.1559110000 & -1.8903090000 \\ \mathrm{H} & -2.1108890000 & 2.4992510000 & -2.4634390000 \\ \mathrm{H} & 2.1328850000 & -2.9515380000 & -1.8331840000 \\ \mathrm{H} & 3.1574920000 & -1.5954200000 & -2.3861030000 \\ \mathrm{H} & -0.5486000000 & -2.6881630000 & 1.3278400000 \\ \mathrm{H} & -0.0789290000 & 2.2025280000 & 1.7015160000 \\ \mathrm{H} & 2.3590230000 & 0.7803050000 & -1.7605740000 \\ \mathrm{H} & -2.2394920000 & -1.0093820000 & -1.8101390000\end{array}$




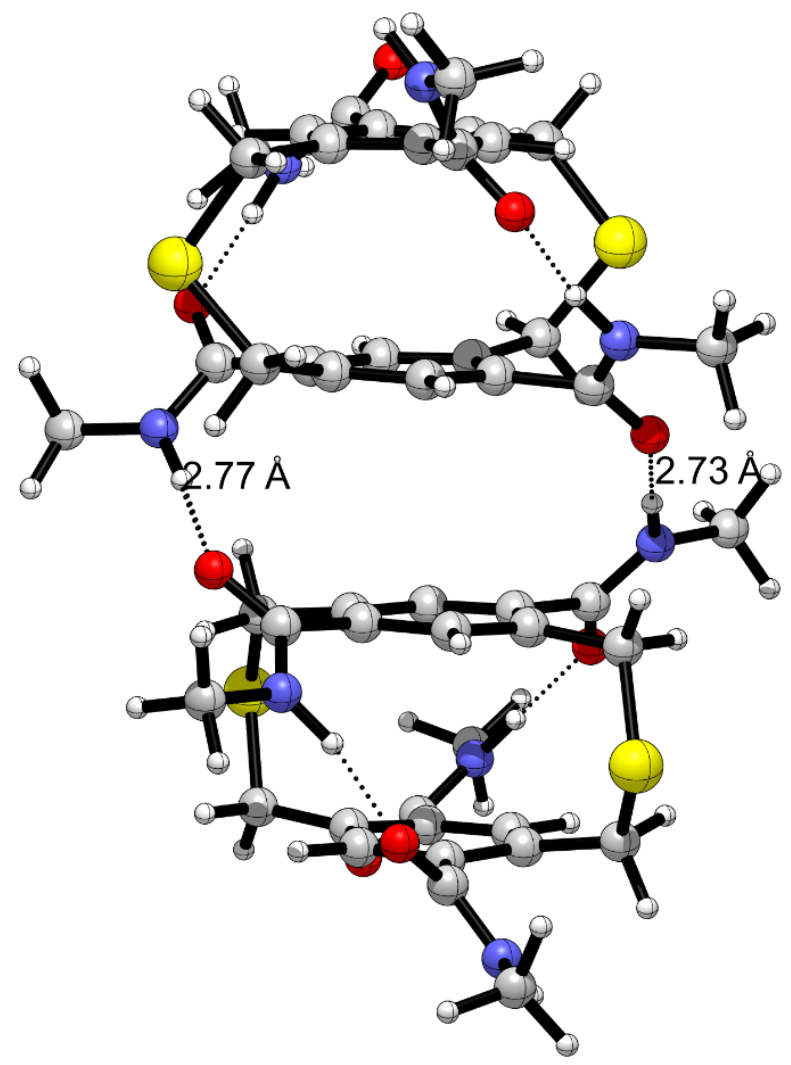

$\left(S_{\mathrm{p}}\right)$-1 chair anti- dimer

Energy: -4493.80982677

$\begin{array}{lrrr}\mathrm{S} & -3.9381870000 & -0.4175690000 & -3.5391390000 \\ \mathrm{~S} & -3.1594190000 & 0.4728900000 & 3.7514900000 \\ \mathrm{~S} & 3.9053480000 & -3.1721980000 & 1.6450690000 \\ \mathrm{~S} & 3.1247150000 & 3.3026630000 & -1.8009950000 \\ \mathrm{O} & -6.0003790000 & 3.4444040000 & 1.5934490000 \\ \mathrm{O} & -2.8826360000 & 2.8795140000 & -2.1809840000 \\ \mathrm{O} & -4.3450900000 & -3.5246610000 & 0.4952160000 \\ \mathrm{O} & -0.5691130000 & -1.5842360000 & 3.0453210000 \\ \mathrm{O} & 0.5582050000 & -0.3344000000 & -3.4333480000 \\ \mathrm{O} & 4.4370240000 & -2.9670840000 & -2.0142880000 \\ \mathrm{O} & 6.0822280000 & 3.7938860000 & -0.0888080000 \\ \mathrm{O} & 3.0129090000 & 1.6504340000 & 3.1156060000 \\ \mathrm{~N} & -6.3273080000 & -3.3681730000 & -0.5914560000 \\ \mathrm{~N} & -1.3007870000 & 1.7119200000 & -3.3259720000 \\ \mathrm{~N} & -4.6590290000 & 3.7021690000 & -0.2390670000 \\ \mathrm{~N} & -2.2024490000 & -2.8881270000 & 2.1712580000 \\ \mathrm{~N} & 1.3536040000 & 0.2249810000 & 3.7351440000 \\ \mathrm{~N} & 6.2978890000 & -3.2972670000 & -0.7696910000 \\ \mathrm{~N} & 4.7584990000 & 3.3249510000 & 1.7168420000 \\ \mathrm{~N} & 2.2029250000 & -1.8677310000 & -3.1512990000 \\ \mathrm{C} & -6.4591580000 & -4.8142750000 & -0.7155360000 \\ \mathrm{C} & -1.3589930000 & 2.6031790000 & -4.4728590000\end{array}$




\begin{tabular}{|c|c|c|c|}
\hline & -2.1042520000 & 1.8891820000 & 2697120000 \\
\hline C & -2.4222150000 & -1.1460560000 & -2.6795380000 \\
\hline & -5.3590330000 & -1.0030200000 & 0000 \\
\hline & -5.2545740000 & -2.8030420000 & 0.0080200000 \\
\hline & -4.7328620000 & 5.1529510000 & -0.1812730000 \\
\hline & -5.3261010000 & 2.9484990000 & 0.6565230000 \\
\hline $\mathrm{C}$ & -5.0462470000 & -0.7641680000 & 1.3691430000 \\
\hline C & -5.2333510000 & -1.3129300000 & 0.0979750000 \\
\hline $\mathrm{C}$ & -5.3404710000 & -0.4712110000 & -1.0241750000 \\
\hline C & -5.3541350000 & 100000 & 620000 \\
\hline $\mathrm{C}$ & -5.2269650000 & 30000 & 320000 \\
\hline $\mathrm{C}$ & -5.0289280000 & 30000 & 000 \\
\hline 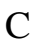 & -4.7430350000 & 580000 & 860000 \\
\hline $\mathrm{C}$ & -1.8233660000 & 1.1913610000 & 2.6221440000 \\
\hline 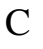 & -1.8560330000 & 20000 & 740000 \\
\hline$c$ & -2.0587440000 & 90000 & -1.3625930000 \\
\hline $\mathrm{C}$ & -1.9 & 20000 & 710000 \\
\hline $\mathrm{C}$ & 540000 & 000000 & 40000 \\
\hline $\mathrm{C}$ & 70000 & 00000 & 30000 \\
\hline $\mathrm{C}$ & 0000 & 00000 & 0000 \\
\hline $\mathrm{C}$ & 0000 & 30000 & 30000 \\
\hline 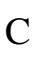 & -1.4 & -1.7 & 30000 \\
\hline $\mathrm{C}$ & 000 & 0000 & 30000 \\
\hline $\mathrm{C}$ & 6.35 & 30000 & 750000 \\
\hline $\mathrm{C}$ & 0000 & 0000 & 0000 \\
\hline $\mathrm{C}$ & 000 & 4.6 & 2.22 \\
\hline C & 5.3 & 2.9 & 000 \\
\hline $\mathrm{C}$ & 0000 & 00000 & 20000 \\
\hline$c$ & 0000 & 20000 & 30000 \\
\hline $\mathrm{C}$ & 0000 & 30000 & 430000 \\
\hline $\mathrm{C}$ & 0000 & 20000 & -2.69 \\
\hline $\mathrm{C}$ & 2.17 & 0.7 & 20000 \\
\hline $\mathrm{C}$ & 0000 & 90000 & 140000 \\
\hline $\mathrm{C}$ & 0000 & 10000 & 880000 \\
\hline C & 2.0315550000 & 10000 & 1.0492170000 \\
\hline $\mathrm{C}$ & 60000 & 0000 & 40000 \\
\hline $\mathrm{C}$ & 0000 & 0000 & 80000 \\
\hline $\mathrm{C}$ & 0000 & 0000 & 50000 \\
\hline $\mathrm{C}$ & 0000 & 0000 & 190000 \\
\hline $\mathrm{C}$ & 4.6754650000 & 2.2985920000 & -2.2210190000 \\
\hline $\mathrm{C}$ & 350000 & 60000 & 360000 \\
\hline $\mathrm{C}$ & 5.3071800000 & 760000 & 0.7179990000 \\
\hline $\mathrm{C}$ & 30000 & 0000 & 960000 \\
\hline $\mathrm{C}$ & 5.2497800000 & 130000 & 0.1434020000 \\
\hline $\mathrm{C}$ & 5.0096550000 & 1.2387740000 & -1.2057610000 \\
\hline C & 5.0265720000 & -0.1037600000 & -1.5802990000 \\
\hline $\mathrm{H}$ & 7.2450050000 & -5.1487240000 & -0.6494740000 \\
\hline
\end{tabular}




\begin{tabular}{|c|c|c|c|}
\hline $\mathrm{H}$ & 6.5384830000 & -4.7876740000 & -2.2454420000 \\
\hline & 5.4807980000 & -5.2230350000 & -0.8919020000 \\
\hline & 7.0191480000 & -2.8906530000 & -0.1978860000 \\
\hline & 4.2561070000 & 4.7457210000 & 3.1421480000 \\
\hline H & 4.4364370000 & 5.3954670000 & 1.4882130000 \\
\hline & 5.8737990000 & 4.9659920000 & 2.4230930000 \\
\hline & 4.1289870000 & 2.6672170000 & 2.1831560000 \\
\hline & 0.6012190000 & 0.1403510000 & 5.6650930000 \\
\hline & 1.2733270000 & 1.7287840000 & 5.2101160000 \\
\hline 1 & 2.3655180000 & 0.3899340000 & 5.5819730000 \\
\hline & 2.7847130000 & -3.1544580000 & -4.6666590000 \\
\hline & 2.1108880000 & -1.6044340000 & -5.2423290000 \\
\hline & 1.0288700000 & -2.8479240000 & -4.6050700000 \\
\hline & 0.6394570000 & -0.4588620000 & 3.4489820000 \\
\hline & 2.9889850000 & -2.2352440000 & 1620000 \\
\hline 1 & 4.5409800000 & 1.8642020000 & -3.2143350000 \\
\hline $\mathrm{H}$ & 5.4506670000 & 3.0654290000 & -2.2489290000 \\
\hline $\boldsymbol{H}$ & 5.1126240000 & -1.3647680000 & 2.7834350000 \\
\hline & 6.1686340000 & -2.4573810000 & 1.8962030000 \\
\hline $\mathrm{H}$ & 0.8411 & 2.6278 & -1.9077290000 \\
\hline H & 1.7873970000 & 1.6189370000 & -2.9874350000 \\
\hline $\mathrm{H}$ & 2.4476460000 & -1.8018230000 & 3.0535220000 \\
\hline 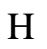 & 1.5571380000 & -2.9396920000 & 2.0564550000 \\
\hline 11 & 5.4466800000 & 0.7861570000 & 2.1301130000 \\
\hline H & 0000 & -0.3 & 220000 \\
\hline H & 1.8350590000 & -2.4577700000 & -0.5830870000 \\
\hline $\mathrm{H}$ & 1.9840310000 & 2.2928000000 & 0.6982730000 \\
\hline$H$ & -2.3606640000 & 2.5940530000 & -4.9125490000 \\
\hline $\mathrm{H}$ & -0.6339220000 & 2.2595820000 & -5.2111470000 \\
\hline $\mathrm{H}$ & 60000 & 3.63 & -4.1842690000 \\
\hline H & -0.6094880000 & 0.9548040000 & -3.3209770000 \\
\hline $\mathrm{H}$ & -2.7947700000 & -4.65 & 3.0691940000 \\
\hline $\mathrm{H}$ & -2.1965960000 & -3.4196000000 & 4.2069920000 \\
\hline $\mathrm{H}$ & -1.0494840000 & -4.3213850000 & 3.2060130000 \\
\hline $\mathrm{H}$ & -2.9605230000 & -3.0206120000 & 1.4962610000 \\
\hline $\mathrm{H}$ & -7.3141930000 & -5.0382930000 & -1.3538020000 \\
\hline $\mathrm{H}$ & -6.6039430000 & -5.2811220000 & 0.2631530000 \\
\hline $\mathrm{H}$ & -5.5548250000 & -5.2353280000 & -1.1601530000 \\
\hline $\mathrm{H}$ & -5.7642020000 & 5.4972160000 & -0.3044080000 \\
\hline $\mathrm{H}$ & -4.1151600000 & 5.5573990000 & -0.9838470000 \\
\hline $\mathrm{H}$ & -4.3725020000 & 5.5276170000 & 0.7823740000 \\
\hline $\mathrm{H}$ & -7.0873400000 & -2.7758290000 & -0.8847090000 \\
\hline $\mathrm{H}$ & -4.0453580000 & 3.2843650000 & -0.9473070000 \\
\hline $\mathrm{H}$ & -2.0025890000 & 2.2658790000 & 2.5386590000 \\
\hline $\mathrm{H}$ & -0.9051800000 & 1.0400650000 & 3.1895810000 \\
\hline 11 & -2.6042500000 & -2.2140670000 & -2.5323050000 \\
\hline $\mathrm{H}$ & -1.6279190000 & -1.0391330000 & -3.4215030000 \\
\hline
\end{tabular}




$\begin{array}{lrrr}\mathrm{H} & -5.5170550000 & 0.8356350000 & 3.6755800000 \\ \mathrm{H} & -4.7129430000 & 2.2337100000 & 2.9857390000 \\ \mathrm{H} & -5.3637940000 & -2.0924960000 & -2.4678140000 \\ \mathrm{H} & -6.2375480000 & -0.6449190000 & -2.9831060000 \\ \mathrm{H} & -1.8744890000 & -2.4029960000 & -0.3949370000 \\ \mathrm{H} & -1.9288470000 & 2.4675950000 & 0.2687020000 \\ \mathrm{H} & -5.4229940000 & 1.5753130000 & -1.6549770000 \\ \mathrm{H} & -4.8743710000 & -1.4315830000 & 2.2078900000\end{array}$

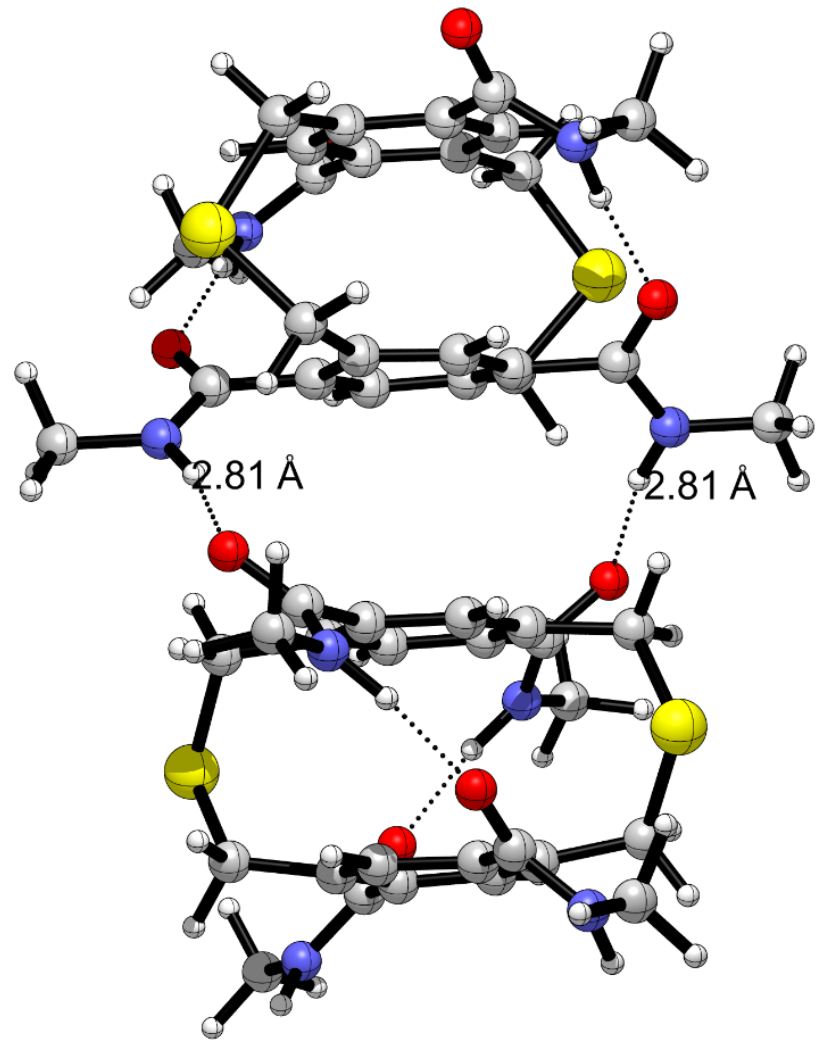

$\left(S_{\mathrm{p}}\right)$-1 chair syn- dimer

Energy: -4493.80054211

$\begin{array}{lccc}\mathrm{S} & -3.6505640000 & -0.1762120000 & 3.6996790000 \\ \mathrm{~S} & -3.6501160000 & 0.1764230000 & -3.6996130000 \\ \mathrm{~S} & 3.4261820000 & 3.3888450000 & -1.4606650000 \\ \mathrm{~S} & 3.4246400000 & -3.3889610000 & 1.4606710000 \\ \mathrm{O} & -6.0882770000 & 3.1329350000 & 1.8813020000 \\ \mathrm{O} & -2.6263910000 & -3.1964690000 & 1.6866390000 \\ \mathrm{O} & -6.0874630000 & -3.1335610000 & -1.8814010000 \\ \mathrm{O} & -2.6272840000 & 3.1965880000 & -1.6863990000 \\ \mathrm{O} & 0.8296950000 & 0.1617620000 & -3.5924320000 \\ \mathrm{O} & 4.3965900000 & 2.9289330000 & 2.1032890000 \\ \mathrm{O} & 4.3950260000 & -2.9295250000 & -2.1033740000 \\ \mathrm{O} & 0.8296240000 & -0.1604430000 & 3.5923390000 \\ \mathrm{~N} & -4.5943510000 & -3.6277900000 & -0.2331100000 \\ \mathrm{~N} & -1.0724320000 & -2.1262040000 & 2.9492140000 \\ \mathrm{~N} & -4.5954030000 & 3.6276500000 & 0.2329770000\end{array}$




\begin{tabular}{|c|c|c|c|}
\hline $\mathrm{N}$ & -1.0724300000 & 2.1274260000 & -2.9487460000 \\
\hline & 2.3883310000 & 1.4716140000 & 3.3476810000 \\
\hline$N$ & 6.0515700000 & -3.5586900000 & -0.6897890000 \\
\hline & 6.0533750000 & 3.5573070000 & 0.6896130000 \\
\hline $\mathrm{N}$ & 2.3872460000 & -1.4713920000 & -3.3477880000 \\
\hline $\mathrm{C}$ & -4.6165030000 & -5.0440890000 & -0.5559690000 \\
\hline $\mathrm{C}$ & -1.0698010000 & -3.1743400000 & 3.9560750000 \\
\hline $\mathrm{C}$ & -1.9142740000 & -2.1774140000 & 1.9051380000 \\
\hline $\mathrm{C}$ & -2.2728200000 & 0.7617210000 & 2.8120720000 \\
\hline $\mathrm{C}$ & -5.2234410000 & 0.4935940000 & 2.8812910000 \\
\hline $\mathrm{C}$ & -4.6181220000 & 5.0439520000 & 940000 \\
\hline $\mathrm{C}$ & -5.3688790000 & 2.7587690000 & 0.9205330000 \\
\hline $\mathrm{C}$ & -5.3681910000 & -2.7591910000 & -0.9206180000 \\
\hline $\mathrm{C}$ & -5.3093800000 & 1.0413440000 & -0.8993650000 \\
\hline $\mathrm{C}$ & -5.3193460000 & 1.3266410000 & 00000 \\
\hline $\mathrm{C}$ & -5.3007460000 & 0.2648210000 & 1.3929440000 \\
\hline $\mathrm{C}$ & -5.3094460000 & -1.0417670000 & 330000 \\
\hline $\mathrm{C}$ & -5.3190650000 & -1.3270590000 & 400000 \\
\hline $\mathrm{C}$ & -5.3003960000 & -0.2652440000 & -1.3930220000 \\
\hline $\mathrm{C}$ & -5.2227510000 & -0.4940490000 & 30000 \\
\hline $\mathrm{C}$ & -2.2721390000 & -0.7611610000 & -2.8119930000 \\
\hline $\mathrm{C}$ & -1.9568880000 & 1.3120440000 & 0.3952720000 \\
\hline $\mathrm{C}$ & -2.0096830000 & 0.3293790000 & 00000 \\
\hline $\mathrm{C}$ & -1.9201910000 & -1.0104260000 & 20000 \\
\hline $\mathrm{C}$ & -1.9567570000 & -1.3115750000 & 10000 \\
\hline $\mathrm{C}$ & -2.0094200000 & -0.3289100000 & -1.3854120000 \\
\hline $\mathrm{C}$ & -1.9201150000 & 1.0108970000 & -0.9677770000 \\
\hline $\mathrm{C}$ & -1.9145640000 & 2.1779620000 & -1.9048660000 \\
\hline $\mathrm{C}$ & -1.0703050000 & 3.1757740000 & 30000 \\
\hline $\mathrm{C}$ & 2.2739120000 & 1.8721490000 & 100000 \\
\hline $\mathrm{C}$ & 6.0700510000 & -4.9238840000 & -1.2014390000 \\
\hline $\mathrm{C}$ & 5.1572680000 & -2.6566830000 & -1.1422090000 \\
\hline $\mathrm{C}$ & 6.0726210000 & 4.9224660000 & 1.2013210000 \\
\hline $\mathrm{C}$ & 5.1586540000 & 2.6557370000 & 00000 \\
\hline $\mathrm{C}$ & 4.8421950000 & 2.2186130000 & -1.8922280000 \\
\hline $\mathrm{C}$ & 1.9059670000 & 2.3122820000 & -1.7896220000 \\
\hline $\mathrm{C}$ & 2.2725230000 & -1.8718200000 & -4.7437520000 \\
\hline $\mathrm{C}$ & 1.6245490000 & -0.4890270000 & -2.8505920000 \\
\hline $\mathrm{C}$ & 1.6249200000 & 0.4897880000 & 2.8505090000 \\
\hline $\mathrm{C}$ & 1.7531260000 & -1.2851150000 & -0.4949200000 \\
\hline $\mathrm{C}$ & 1.7340480000 & -0.2081490000 & -1.3848850000 \\
\hline $\mathrm{C}$ & 1.7803320000 & 1.1078240000 & -0.8888270000 \\
\hline $\mathrm{C}$ & 1.7537880000 & 1.2857810000 & 0.4948170000 \\
\hline $\mathrm{C}$ & 1.7341930000 & 0.2088210000 & 1.3847880000 \\
\hline $\mathrm{C}$ & 1.7798100000 & -1.1071740000 & 0.8887360000 \\
\hline $\mathrm{C}$ & 1.9048540000 & -2.3117280000 & 1.7894850000 \\
\hline $\mathrm{C}$ & 4.8411490000 & -2.2193410000 & 1.8921970000 \\
\hline
\end{tabular}




\begin{tabular}{|c|c|c|c|}
\hline $\mathrm{C}$ & 5.1145720000 & -0.2081090000 & -1.3580430000 \\
\hline & 5.0793000000 & 1.1077620000 & -0.8928920000 \\
\hline$C$ & 5.1555150000 & 1.3055380000 & 0.4975550000 \\
\hline $\mathrm{C}$ & 5.1147680000 & 0.2072010000 & 1.3579410000 \\
\hline $\mathrm{C}$ & 5.0787750000 & -1.1086510000 & 0.8927940000 \\
\hline $\mathrm{C}$ & 5.1547980000 & -1.3064780000 & -0.4976590000 \\
\hline $\mathrm{H}$ & 1.2688330000 & 2.2457100000 & 4.9594740000 \\
\hline 1 & 3.0036080000 & 2.6596740000 & 4.9318590000 \\
\hline $\mathrm{H}$ & 2.4648160000 & 1.0253080000 & 5.4087030000 \\
\hline $\mathrm{H}$ & 3.0734380000 & 1.9659160000 & 2.7678530000 \\
\hline $\mathrm{H}$ & 1.2671080000 & -2.2444530000 & -4.9596260000 \\
\hline $\mathrm{H}$ & 3.0015100000 & -2.6600090000 & -4.9319440000 \\
\hline $\mathrm{H}$ & 2.4642270000 & -1.0251550000 & -5.4088110000 \\
\hline I & 3.0721790000 & -1.9660110000 & -2.7680350000 \\
\hline $\mathrm{H}$ & 5.1022940000 & 5.3970270000 & 1.0335550000 \\
\hline $\mathrm{H}$ & 6.8466800000 & 5.4844280000 & 0.6786810000 \\
\hline $\mathrm{H}$ & 6.2779900000 & 4.9299430000 & 2.2747190000 \\
\hline $\mathrm{H}$ & 5.1008560000 & -5.3994860000 & -1.0299850000 \\
\hline H & 6.8467660000 & -5.4850470000 & -0.6818920000 \\
\hline $\mathrm{H}$ & 6.2712570000 & -4.9309910000 & -2.2756040000 \\
\hline $\mathrm{H}$ & 6.7225730000 & 3.2837430000 & -0.0102290000 \\
\hline $\mathrm{H}$ & 6.7205780000 & -3.2856310000 & 0.0104280000 \\
\hline $\mathrm{H}$ & 1.9063250000 & -2.0391330000 & 2.8426810000 \\
\hline $\mathrm{H}$ & 1.0767820000 & -3.0071620000 & 1.6337050000 \\
\hline $\mathrm{H}$ & 300000 & 3.0080340000 & -1.6339820000 \\
\hline $\mathrm{H}$ & 1.9074470000 & 2.0396080000 & -2.8427980000 \\
\hline $\mathrm{H}$ & 4.6368350000 & -1.7925410000 & 2.8771940000 \\
\hline $\mathrm{H}$ & 5.6961710000 & -2.8908040000 & 2.0019830000 \\
\hline $\mathrm{H}$ & 5.6975220000 & 2.8897010000 & -2.0019030000 \\
\hline $\mathrm{H}$ & 4.6377150000 & 1.7919850000 & -2.8772650000 \\
\hline $\mathrm{H}$ & 1.7685810000 & 2.2967790000 & 0.8891480000 \\
\hline $\mathrm{H}$ & 1.7673320000 & -2.2961210000 & -0.8892450000 \\
\hline $\mathrm{H}$ & 5.0546400000 & -0.3970770000 & -2.4254110000 \\
\hline $\mathrm{H}$ & 5.0550050000 & 0.3961960000 & 2.4253160000 \\
\hline $\mathrm{H}$ & -5.6117740000 & -5.4696370000 & -0.3928760000 \\
\hline $\mathrm{H}$ & -3.8947210000 & -5.5508510000 & 0.0861460000 \\
\hline $\mathrm{H}$ & -4.3571290000 & -5.2113220000 & -1.6065620000 \\
\hline $\mathrm{H}$ & -3.9454220000 & -3.3236340000 & 0.4975250000 \\
\hline $\mathrm{H}$ & -5.6138060000 & 5.4688580000 & 0.3935750000 \\
\hline $\mathrm{H}$ & -3.8972220000 & 5.5511160000 & -0.0869920000 \\
\hline $\mathrm{H}$ & -4.3579040000 & 5.2114280000 & 1.6061440000 \\
\hline $\mathrm{H}$ & -3.9465510000 & 3.3238030000 & -0.4978500000 \\
\hline $\mathrm{H}$ & -2.0549130000 & -3.2542950000 & 4.4256650000 \\
\hline $\mathrm{H}$ & -0.3261590000 & -2.9200040000 & 4.7123750000 \\
\hline $\mathrm{H}$ & -0.8273790000 & -4.1447770000 & 3.5121220000 \\
\hline $\mathrm{H}$ & -2.0556970000 & 3.2559430000 & -4.4243620000 \\
\hline $\mathrm{H}$ & -0.3271570000 & 2.9214860000 & -4.7122140000 \\
\hline
\end{tabular}




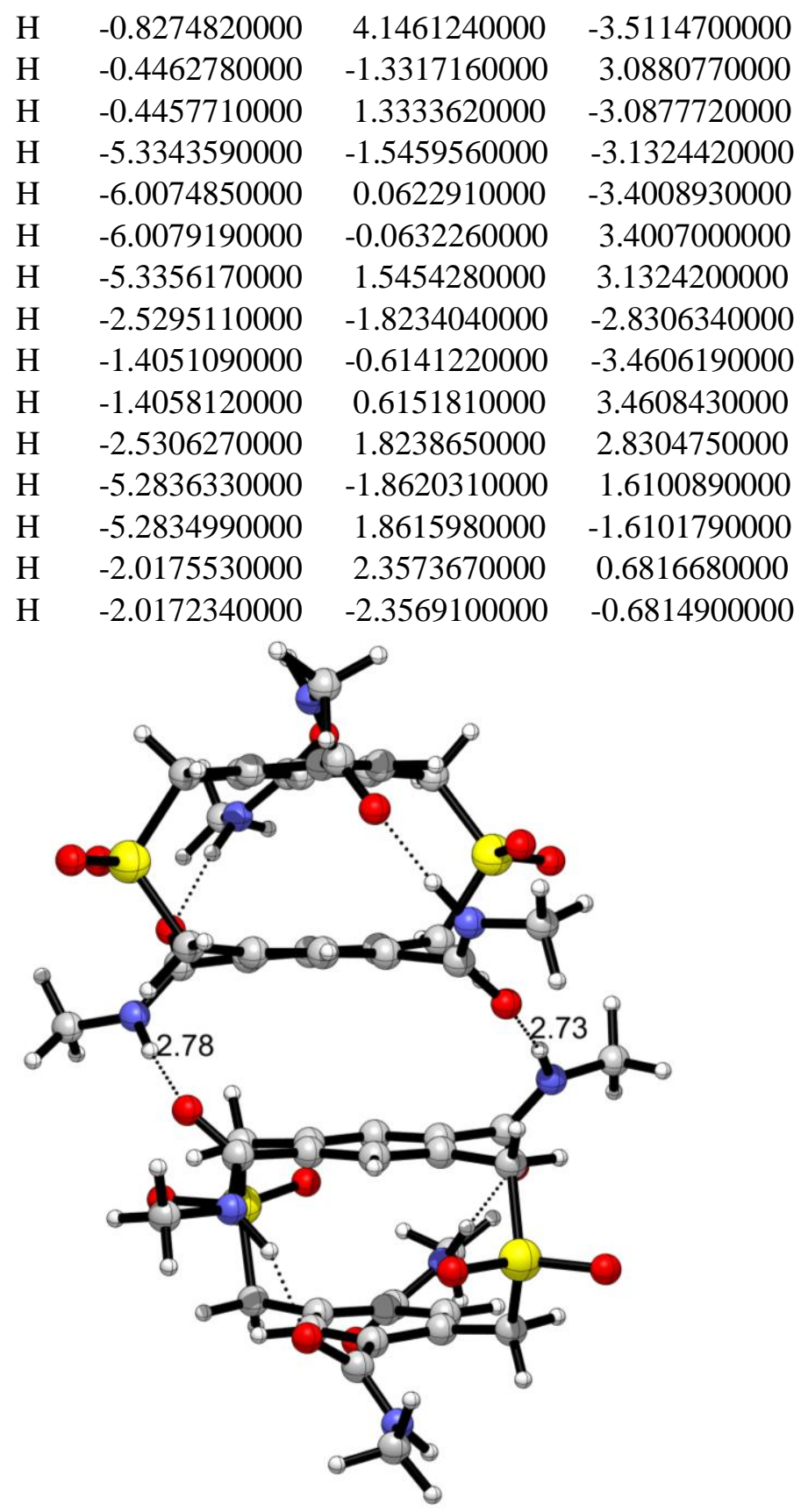

$\left(S_{\mathrm{p}}\right)$-2 chair anti- dimer

Energy: -5094.48113661

$\begin{array}{lrrr}\mathrm{S} & 4.2020660000 & -3.4621070000 & -0.1441220000 \\ \mathrm{~S} & 2.7144420000 & 3.7649480000 & -0.0096680000 \\ \mathrm{~S} & -4.3481710000 & 1.0678140000 & -3.2707300000 \\ \mathrm{~S} & -2.8694810000 & -1.3252360000 & 3.5554060000 \\ \mathrm{O} & 4.6289030000 & -4.8938610000 & 0.5061140000 \\ \mathrm{O} & 4.0098550000 & -3.3433090000 & -1.7627020000 \\ \mathrm{O} & 2.3378800000 & 5.1972980000 & -0.6901560000 \\ \mathrm{O} & 2.6552730000 & 3.5896640000 & 1.6141070000 \\ \mathrm{O} & -4.6558850000 & 0.8154760000 & -4.8511240000\end{array}$




\begin{tabular}{|c|c|c|c|}
\hline ) & -4.4152730000 & 2.5786440000 & -2.6575200000 \\
\hline C & -2.4532910000 & -1.2485050000 & 5.1300090000 \\
\hline & -3.0283550000 & -2.7919790000 & 0000 \\
\hline & -5.6687540000 & 2.1851980000 & 3.3145070000 \\
\hline & -2.7502530000 & & 170000 \\
\hline & -4.3957530000 & -3.3581600000 & 7070000 \\
\hline C & -0.2809570000 & -2.8891220000 & 6770000 \\
\hline & 30000 & 1.1683490000 & 0000 \\
\hline $\mathrm{O}$ & 90000 & -1.0371000000 & 980000 \\
\hline ) & 5.9151220000 & 3.4 & 0000 \\
\hline $\mathrm{O}$ & 3.0620490000 & -0.19514 & 40000 \\
\hline 1 & -6.5689300000 & -2.8 & 0000 \\
\hline & 10000 & 6260000 & 750000 \\
\hline & 90000 & & 160000 \\
\hline & 7130000 & -3.6 & 290000 \\
\hline & 1.4 & -1.8 & 0000 \\
\hline & 6.5 & -1.9 & -2 \\
\hline & 00000 & 2.0 & 0000 \\
\hline 1 & 2.15 & -0.0 & 40000 \\
\hline $\mathrm{C}$ & 120000 & -4.0 & 730000 \\
\hline & -1.42 & & -2.6 \\
\hline C & -2.1 & & -1. \\
\hline $\mathrm{C}$ & 30000 & 0.31 & 630000 \\
\hline $\mathrm{C}$ & -5.6 & & 00000 \\
\hline & 90000 & -2.5 & 90000 \\
\hline $\mathrm{C}$ & -4.51 & 4.55 & 70000 \\
\hline $\mathrm{C}$ & -5.1 & & 000 \\
\hline $\mathrm{C}$ & 80000 & -1.4 & 10000 \\
\hline $\mathrm{C}$ & -5.3 & -1.3 & 090000 \\
\hline & -5.4 & -0.0 & 60000 \\
\hline $\mathrm{C}$ & -5.3 & 1.0 & 10000 \\
\hline $\mathrm{C}$ & -5.1 & & 0000 \\
\hline $\mathrm{C}$ & -4.9 & -0.2 & 50000 \\
\hline $\mathrm{C}$ & -4.5 & -0.4 & 30000 \\
\hline $\mathrm{C}$ & -1.5 & -0.3 & 50000 \\
\hline$c$ & -1.9 & -1.0 & 60000 \\
\hline $\mathrm{C}$ & -2.1968580000 & 0.20 & 430000 \\
\hline $\mathrm{C}$ & -2.0 & 1.3 & 210000 \\
\hline $\mathrm{C}$ & 30000 & 1.07 & 760000 \\
\hline $\mathrm{C}$ & -1.65 & -0.20 & 0000 \\
\hline $\mathrm{C}$ & 10000 & -1.25 & 800000 \\
\hline $\mathrm{C}$ & -1.726 & -5.06 & 9540000 \\
\hline $\mathrm{C}$ & 230000 & -2.68 & 0.7332550000 \\
\hline $\mathrm{C}$ & 1.4400180000 & 5650000 & 4.7255590000 \\
\hline $\mathrm{C}$ & 6.74 & -2.8 & 30000 \\
\hline C & 5.4760610000 & -1.1390810000 & -2.4937390000 \\
\hline $\mathrm{C}$ & 4.7879560000 & 2.9594560000 & 4.3785020000 \\
\hline
\end{tabular}




$\begin{array}{lrrr}\mathrm{C} & 5.2880170000 & 2.4211450000 & 2.0728370000 \\ \mathrm{C} & 5.5943140000 & -2.2728980000 & 0.4113630000 \\ \mathrm{C} & 2.5532210000 & -2.9612370000 & 0.6474430000 \\ \mathrm{C} & 1.9221500000 & 0.1405670000 & -5.1326460000 \\ \mathrm{C} & 1.3343880000 & 0.4461920000 & -2.7932600000 \\ \mathrm{C} & 2.2472400000 & -0.8628060000 & 2.7875160000 \\ \mathrm{C} & 1.6293510000 & 0.1274070000 & -1.3580270000 \\ \mathrm{C} & 1.9075250000 & -1.1941590000 & -1.0029780000 \\ \mathrm{C} & 2.1547490000 & -1.5563900000 & 0.3255040000 \\ \mathrm{C} & 2.0565710000 & -0.5742580000 & 1.3234640000 \\ \mathrm{C} & 1.8004790000 & 0.7503200000 & 0.9681280000 \\ \mathrm{C} & 1.6100970000 & 1.1247730000 & -0.3652740000 \\ \mathrm{C} & 1.4256750000 & 2.5684780000 & -0.7207160000 \\ \mathrm{C} & 4.4683690000 & 3.3283420000 & -0.6355790000 \\ \mathrm{C} & 5.2937420000 & -0.3280940000 & -1.2470690000 \\ \mathrm{C} & 5.4214830000 & -0.8245990000 & 0.0653910000 \\ \mathrm{C} & 5.3616220000 & 0.0887040000 & 1.1269500000 \\ \mathrm{C} & 5.1422760000 & 1.4513340000 & 0.9281620000 \\ \mathrm{C} & 4.8634940000 & 1.9122900000 & -0.3717060000 \\ \mathrm{C} & 4.9531460000 & 1.0137950000 & -1.4343140000 \\ \mathrm{H} & 7.6794220000 & -3.4122450000 & -3.5045060000 \\ \mathrm{H} & 6.7818430000 & -2.3511550000 & -4.6205050000 \\ \mathrm{H} & 5.4917680000 & -0.2696560000 & 2.1426020000 \\ \mathrm{H} & 5.9073350000 & -3.5649020000 & -3.6555850000 \\ \mathrm{H} & 7.2908620000 & -1.8328410000 & -1.8639610000 \\ \mathrm{H} & 4.3312140000 & 2.4639880000 & 5.2356720000 \\ \mathrm{H} & 4.2320680000 & 3.8715300000 & 4.1389850000 \\ \mathrm{H} & 5.8163330000 & 3.2378870000 & 4.6213420000 \\ \mathrm{H} & 4.1374450000 & 1.2386550000 & 3.2926920000 \\ \mathrm{H} & 0.4228800000 & -2.0444660000 & 5.1131210000 \\ \mathrm{H} & 2.1080660000 & -1.4782820000 & 5.2546320000 \\ \mathrm{H} & 1.7697170000 & -3.1873350000 & 4.8862920000 \\ \mathrm{H} & 2.7599360000 & -0.2937490000 & -5.6770180000 \\ \mathrm{H} & 1.8463110000 & 1.2076500000 & -5.3599710000 \\ \mathrm{H} & 0.9916580000 & -0.3381450000 & -5.4504230000 \\ \mathrm{H} & 0.8042910000 & -2.3055390000 & 2.6793150000 \\ \mathrm{H} & 3.0341150000 & -0.5365040000 & -3.4496870000 \\ \mathrm{H} & 5.4194410000 & 3.5853970000 & -1.6939870000 \\ \mathrm{H} & 5.6327610000 & -2.4380260000 & 1.4893460000 \\ \mathrm{H} & 1.4366870000 & 2.7720900000 & -1.7889370000 \\ \mathrm{H} & 1.8730710000 & -3.7098120000 & 0.2389130000 \\ \mathrm{H} & -1.950000000000 & -2.7736870000 & -0.0480980000 \\ \mathrm{H} & -1.9524550000 & -1.7755130000\end{array}$




$\begin{array}{crrr}\mathrm{H} & 1.8037300000 & 1.5177880000 & 1.7360730000 \\ \mathrm{H} & -2.4583710000 & 4.8351010000 & -2.7004810000 \\ \mathrm{H} & -1.0098350000 & 4.4829010000 & -3.6769830000 \\ \mathrm{H} & -0.8360210000 & 5.1373440000 & -2.0289940000 \\ \mathrm{H} & -0.7779020000 & 2.4259150000 & -2.5903920000 \\ \mathrm{H} & -2.4193390000 & -5.7140070000 & 0.1038000000 \\ \mathrm{H} & -1.8753510000 & -5.1558060000 & 1.7123740000 \\ \mathrm{H} & -0.6999750000 & -5.3526810000 & 0.3970200000 \\ \mathrm{H} & -2.8468660000 & -3.4966290000 & -0.2909060000 \\ \mathrm{H} & -7.5514280000 & -3.8889240000 & -3.1941900000 \\ \mathrm{H} & -7.0888450000 & -4.9172860000 & -1.8121720000 \\ \mathrm{H} & -5.8482230000 & -4.3442610000 & -2.9400300000 \\ \mathrm{H} & -5.5351370000 & 4.9331860000 & 2.5575300000 \\ \mathrm{H} & -3.9246860000 & 5.2731360000 & 1.8675590000 \\ \mathrm{H} & -4.0853250000 & 4.4061760000 & 3.4202070000 \\ \mathrm{H} & -7.3588450000 & -2.2780720000 & -1.4503570000 \\ \mathrm{H} & -3.9792250000 & 3.2819520000 & 0.8094600000 \\ \mathrm{H} & -1.5320670000 & 0.6304140000 & 3.1843750000 \\ \mathrm{H} & -0.6198950000 & -0.8840620000 & 2.9812490000 \\ \mathrm{H} & -2.7051130000 & -0.6625680000 & -3.4661680000 \\ \mathrm{H} & -1.9932210000 & 0.9590800000 & -3.5996880000 \\ \mathrm{H} & -5.2031610000 & -1.1295770000 & 3.8793610000 \\ \mathrm{H} & -4.4696390000 & 0.4883300000 & 3.8814160000 \\ \mathrm{H} & -5.7412260000 & -0.8531520000 & -2.8912540000 \\ \mathrm{H} & -6.5606980000 & 0.7002110000 & -2.5952510000 \\ \mathrm{H} & -2.0203200000 & -1.9232550000 & -1.7061430000 \\ \mathrm{H} & -1.8328910000 & 1.9350880000 & 1.3311270000 \\ \mathrm{H} & -5.5123670000 & 2.0602970000 & -0.5034430000 \\ \mathrm{H} & -4.8357400000 & -2.4023860000 & 1.5137070000\end{array}$




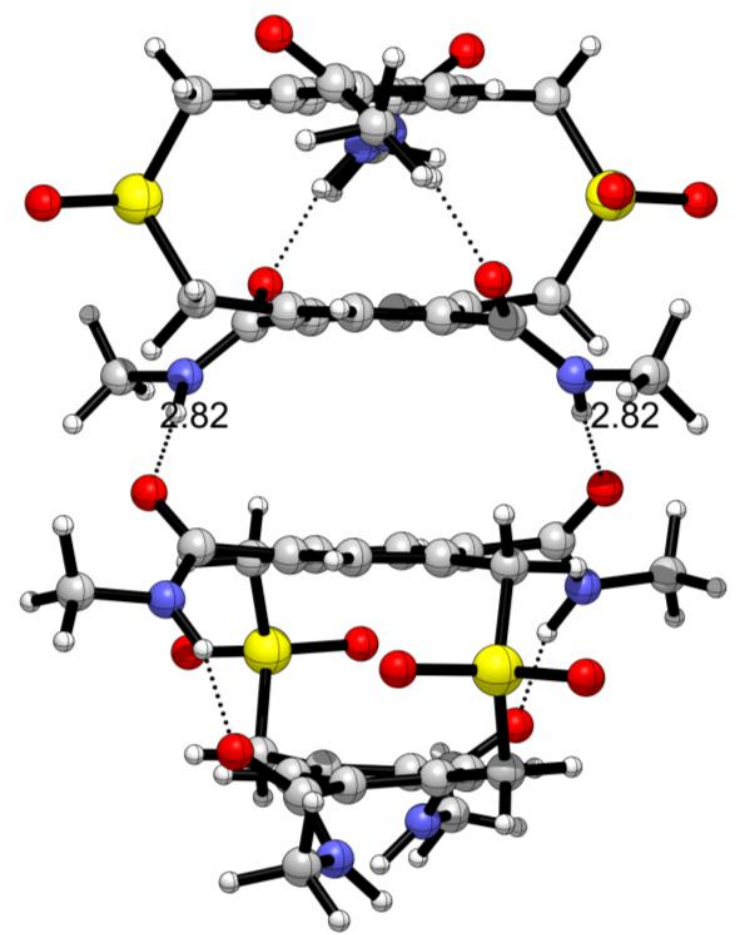

$\left(S_{\mathrm{p}}\right)$-2 chair syn- dimer

Energy: -5094.48113661

$\begin{array}{lrrr}\mathrm{S} & -3.7743840000 & 1.5546170000 & -3.3503640000 \\ \mathrm{~S} & -3.7724130000 & -1.5554770000 & 3.3505240000 \\ \mathrm{~S} & 3.4247380000 & 3.6809190000 & 0.0535570000 \\ \mathrm{~S} & 3.4268910000 & -3.6804410000 & -0.0534890000 \\ \mathrm{O} & -3.7084950000 & 1.5669060000 & -4.9786440000 \\ \mathrm{O} & -3.8060740000 & 2.9766240000 & -2.5430960000 \\ \mathrm{O} & -3.7058950000 & -1.5680940000 & 4.9787790000 \\ \mathrm{O} & -3.8039250000 & -2.9773170000 & 2.5429650000 \\ \mathrm{O} & 3.4906750000 & 5.1771180000 & -0.5827010000 \\ \mathrm{O} & 3.3607070000 & 3.4889970000 & 1.6747720000 \\ \mathrm{O} & 3.4937670000 & -5.1764840000 & 0.5829960000 \\ \mathrm{O} & 3.3618870000 & -3.4887640000 & -1.6747030000 \\ \mathrm{O} & -6.1729280000 & 2.1224790000 & 2.9452660000 \\ \mathrm{O} & -2.4507220000 & -3.6392610000 & -0.0527330000 \\ \mathrm{O} & -6.1730790000 & -2.1239950000 & -2.9439240000 \\ \mathrm{O} & -2.4523630000 & 3.6391120000 & 0.0527520000 \\ \mathrm{O} & 0.7862330000 & 1.6380550000 & -3.1478490000 \\ \mathrm{O} & 4.6422100000 & 1.4224050000 & 3.3369160000 \\ \mathrm{O} & 4.6411760000 & -1.4216990000 & -3.3378350000 \\ \mathrm{O} & 0.7875450000 & -1.6380780000 & 3.1487280000 \\ \mathrm{~N} & -4.6789690000 & -3.2616630000 & -1.6494890000 \\ \mathrm{~N} & -0.9968170000 & -3.2246980000 & 1.6481280000 \\ \mathrm{~N} & -4.6798350000 & 3.2607990000 & 1.6502280000 \\ \mathrm{~N} & -0.9988860000 & 3.2253170000 & -1.6486250000 \\ \mathrm{~N} & 2.3778870000 & -0.1035360000 & 3.6818550000\end{array}$




\begin{tabular}{|c|c|c|c|}
\hline & 6.2210120000 & -2.7348870000 & 000 \\
\hline & 6.2204790000 & 2.7369030000 & 23671870000 \\
\hline & .3756890000 & .1028580000 & -3.6816200000 \\
\hline & -4.7211080000 & -4.4337420000 & -2.5108030000 \\
\hline & -0.9965430000 & -4.6106490000 & 2.0985950000 \\
\hline & -1.8326750000 & -2.8233830000 & 0.6790170000 \\
\hline $\mathrm{C}$ & -2.2148880000 & -0.5703620000 & 50000 \\
\hline $\mathrm{C}$ & -5.3476730000 & -0.6311220000 & 2.8376060000 \\
\hline & -4.7221300000 & 4.4328640000 & 2.5115510000 \\
\hline$c$ & -5.4426090000 & 2.1859540000 & 0000 \\
\hline $\mathrm{C}$ & -5.4422750000 & -2.1871280000 & 50000 \\
\hline$c$ & 210000 & 450000 & 10000 \\
\hline $\mathrm{C}$ & -5.3786910000 & 460000 & 0000 \\
\hline $\mathrm{C}$ & -5.3746250000 & -0.2891870000 & 90000 \\
\hline $\mathrm{C}$ & -5.38 & -1.3 & 0000 \\
\hline $\mathrm{C}$ & -5.378 & 2170000 & 40000 \\
\hline C & -5.37 & 0000 & 0000 \\
\hline $\mathrm{C}$ & -5.3491450000 & 3870000 & 10000 \\
\hline $\mathrm{C}$ & -2.216 & 0.5 & -2.8 \\
\hline $\mathrm{C}$ & -1.98 & & 000 \\
\hline $\mathrm{C}$ & -2.017 & 80000 & 0000 \\
\hline $\mathrm{C}$ & -1.91 & -1. & 20000 \\
\hline $\mathrm{C}$ & -1.9809730000 & 50000 & 30000 \\
\hline $\mathrm{C}$ & -2.018 & 0000 & 0000 \\
\hline $\mathrm{C}$ & -1.92 & & 000 \\
\hline $\mathrm{C}$ & -1.83 & 0000 & 0000 \\
\hline $\mathrm{C}$ & -0.99 & 4.6 & 0000 \\
\hline$C$ & 2.225 & 0000 & 70000 \\
\hline $\mathrm{C}$ & 6.217 & 0000 & 0000 \\
\hline $\mathrm{C}$ & 5.33 & -1.7 & 000 \\
\hline $\mathrm{C}$ & 6.21 & 3.6 & 000 \\
\hline $\mathrm{C}$ & 5.33 & 1.7 & 000 \\
\hline $\mathrm{C}$ & 5.028 & 000 & 0000 \\
\hline $\mathrm{C}$ & 1.896 & 2.8 & 0000 \\
\hline $\mathrm{C}$ & 2.222 & 0000 & 0000 \\
\hline $\mathrm{C}$ & 1.62 & 10000 & 70000 \\
\hline $\mathrm{C}$ & 1.62 & -0.7 & 0000 \\
\hline $\mathrm{C}$ & 1.774 & -0.9 & -0.9 \\
\hline $\mathrm{C}$ & 1.766 & 0000 & 90000 \\
\hline $\mathrm{C}$ & 1.8065670000 & 080000 & 170000 \\
\hline $\mathrm{C}$ & 1.774 & 80000 & 0000 \\
\hline $\mathrm{C}$ & 1.7672150000 & -0.3826460000 & 70000 \\
\hline $\mathrm{C}$ & 1.80 & .0000 & 0000 \\
\hline $\mathrm{C}$ & 1.8984610000 & -2.8268120000 & 0.6798270000 \\
\hline $\mathrm{C}$ & 5.0304420000 & -2.8497370000 & 0.5714670000 \\
\hline $\mathrm{C}$ & 5.2059170000 & 0.4863070000 & -1.2826030000 \\
\hline $\mathrm{C}$ & 5.1883010000 & 1.4029560000 & -0.2270820000 \\
\hline
\end{tabular}




\begin{tabular}{|c|c|c|c|}
\hline C & 70860000 & 0000 & 000 \\
\hline C & 5.2069590000 & -0.4849210000 & 1.2812980000 \\
\hline $\mathrm{C}$ & 5.1893770000 & -1.4015750000 & 0.2257880000 \\
\hline C & 5.2670990000 & -0.8939030000 & -1.0866280000 \\
\hline $\mathrm{H}$ & 1.2110040000 & -0.1025680000 & 5.4388300000 \\
\hline $\mathrm{H}$ & 2.9418120000 & 0.2807520000 & 5.6401240000 \\
\hline $\mathrm{H}$ & 2.4082440000 & -1.3989170000 & 5.3494340000 \\
\hline $\mathrm{H}$ & 3.1257960000 & 0.5388920000 & 3.3967100000 \\
\hline $\mathrm{H}$ & 1.2080430000 & 0.1014450000 & -5.4380880000 \\
\hline $\mathrm{H}$ & 2.9388790000 & -0.2812920000 & -5.6401260000 \\
\hline $\mathrm{H}$ & 2.4048750000 & 1.3982090000 & -5.3492330000 \\
\hline $\mathrm{H}$ & 3.1239760000 & -0.5392530000 & -3.3967810000 \\
\hline $\mathrm{H}$ & 5.2301720000 & 4.1603980000 & 3.5301300000 \\
\hline $\mathrm{H}$ & 6.9707480000 & 4.4578850000 & 3.2744180000 \\
\hline $\mathrm{H}$ & 6.4365 & 860000 & 0000 \\
\hline $\mathrm{H}$ & 5.2302960000 & -4.1571250000 & 9280000 \\
\hline $\mathrm{H}$ & 6.9701080000 & 760000 & 1760000 \\
\hline $\mathrm{H}$ & 6.4389480000 & -3.1956950000 & -4.4176100000 \\
\hline $\mathrm{H}$ & 6.8877250000 & 2.8465690000 & 430000 \\
\hline $\mathrm{H}$ & 6.8890 & 80000 & .0000 \\
\hline $\mathrm{H}$ & 1.9472740000 & -3.0473680000 & 840000 \\
\hline $\mathrm{H}$ & 1.0982410000 & 550000 & 560000 \\
\hline $\mathrm{H}$ & 1.0960330000 & 3.4225420000 & -0.2347200000 \\
\hline $\mathrm{H}$ & 1.9447290000 & 3.0473380000 & 3710000 \\
\hline $\mathrm{H}$ & 4.99341 & -3.0 & 0000 \\
\hline $\mathrm{H}$ & 5.7654700000 & -3.5272950000 & 0.1360790000 \\
\hline $\mathrm{H}$ & 5.7632 & 500000 & +20000 \\
\hline $\mathrm{H}$ & 4.9900380000 & 3.0211850000 & -1.6495500000 \\
\hline $\mathrm{H}$ & 1.7805470000 & 1.7250420000 & 1.7689980000 \\
\hline $\mathrm{H}$ & 1.7810 & 2820000 & 60000 \\
\hline $\mathrm{H}$ & 5.1569740000 & 880000 & 00000 \\
\hline $\mathrm{H}$ & 5.1588 & 370000 & 20000 \\
\hline $\mathrm{H}$ & -5.7088360000 & -4.9030370000 & -2.4824240000 \\
\hline $\mathrm{H}$ & -3.9709320000 & -5.1403810000 & -2.1553950000 \\
\hline $\mathrm{H}$ & -4.5123990000 & -4.1631170000 & -3.5504800000 \\
\hline $\mathrm{H}$ & -3.9862770000 & -3.2742280000 & -0.8966930000 \\
\hline $\mathrm{H}$ & -5.7099360000 & 0050000 & 2.4832510000 \\
\hline $\mathrm{H}$ & -3.9721040000 & 5.1396450000 & 2.1561010000 \\
\hline $\mathrm{H}$ & -4.5133040000 & 4.1622770000 & 3.5512130000 \\
\hline $\mathrm{H}$ & -3.9873440000 & 3.2736020000 & 0.8972360000 \\
\hline $\mathrm{H}$ & -1.9845700000 & -4.8628050000 & 2.4929520000 \\
\hline $\mathrm{H}$ & -0.2487230000 & -4.7151540000 & 2.8858400000 \\
\hline $\mathrm{H}$ & -0.7593960000 & -5.2898240000 & 1.2754030000 \\
\hline $\mathrm{H}$ & -1.9870170000 & 4.8635580000 & -2.4928790000 \\
\hline $\mathrm{H}$ & -0.2510940000 & 4.7165040000 & -2.8856360000 \\
\hline $\mathrm{H}$ & -0.7621140000 & 5.2903170000 & -1.2750150000 \\
\hline $\mathrm{H}$ & -0.4312470000 & -2.5561090000 & 2.1688130000 \\
\hline
\end{tabular}




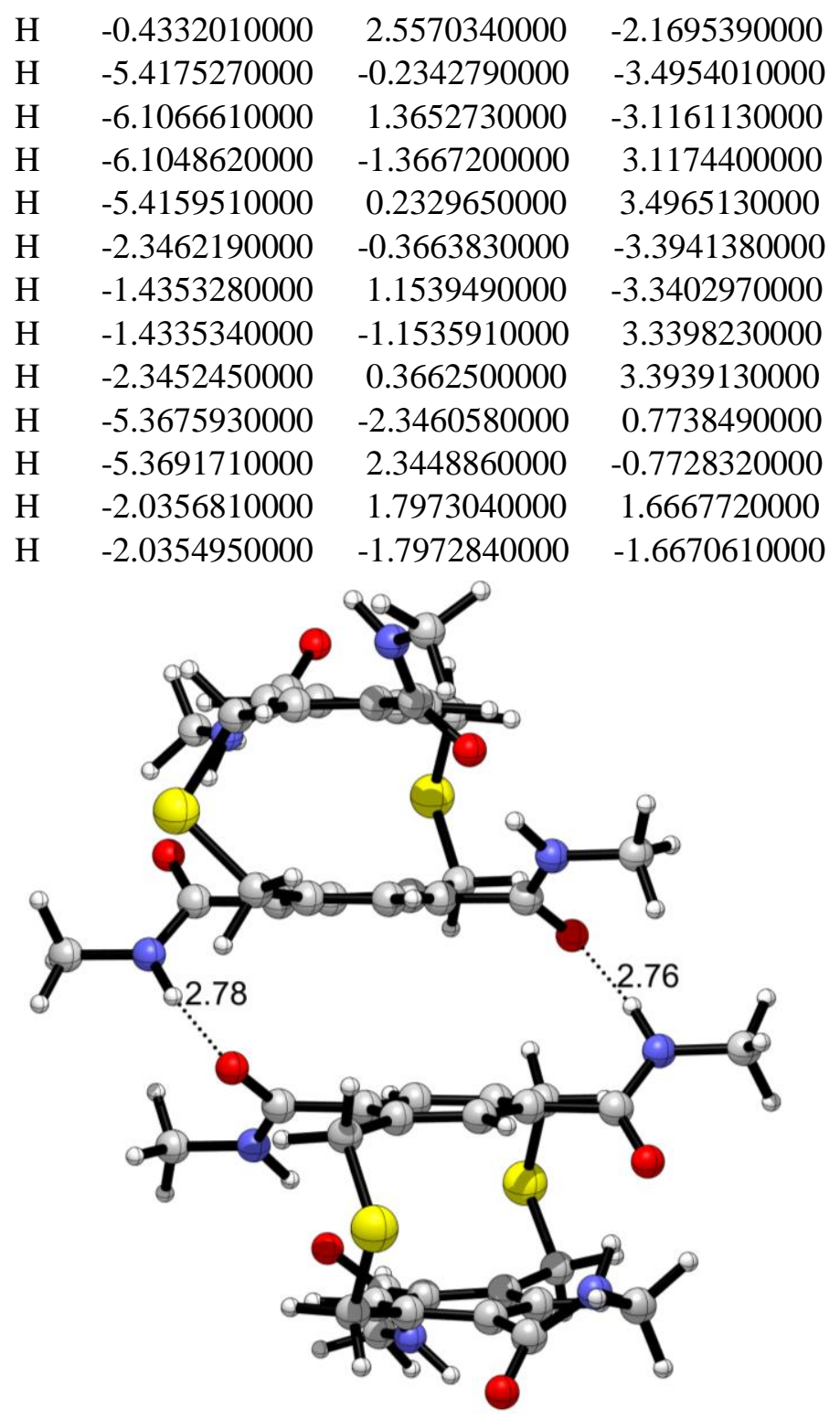

Heterochiral 1 dimer $S_{\mathrm{p}}$ (top) $\cdots R_{\mathrm{p}}$ (bottom)

Energy: -4493.804219

$\begin{array}{lrrr}\text { S } & -3.9171600000 & -0.0348970000 & 3.5244140000 \\ \text { S } & -3.8322790000 & -1.1924870000 & -3.4899360000 \\ \text { S } & 3.9439340000 & 1.3416570000 & 3.4485970000 \\ \text { S } & 3.2322480000 & -1.7498100000 & -3.2102210000 \\ \text { O } & -0.2883490000 & 1.6543270000 & -2.8305100000 \\ \text { O } & -3.9335320000 & -3.0241870000 & 1.3018150000 \\ \text { O } & -3.2056750000 & 3.9457160000 & 0.4351350000 \\ \text { O } & -6.9912540000 & -1.5617340000 & -2.2707680000 \\ \text { O } & 3.2969290000 & -3.9132860000 & -0.2205370000 \\ \text { O } & 7.1077860000 & 1.7107810000 & 2.1964720000 \\ \text { O } & 0.3011120000 & -1.4759270000 & 2.8140020000 \\ \text { O } & 4.0579180000 & 3.0020610000 & -1.4581940000\end{array}$




\begin{tabular}{|c|c|c|c|}
\hline $\mathrm{N}$ & -1.3853790000 & 3.1233690000 & -1.4805350000 \\
\hline & -2.1721270000 & -2.7324510000 & 2.7054320000 \\
\hline$N$ & -5.1757740000 & 4.1285550000 & 1.5508400000 \\
\hline & -5.8805880000 & -2.7617970000 & -0.6681360000 \\
\hline $\mathrm{N}$ & 5.1321980000 & -4.0186100000 & -1.5413760000 \\
\hline $\mathrm{N}$ & 5.9891500000 & 2.8562320000 & 0.5630870000 \\
\hline N & 1.4069680000 & -2.9932060000 & 1.5218860000 \\
\hline $\mathrm{N}$ & 2.2064170000 & 2.8253630000 & -2.7566110000 \\
\hline C & 0.9461600000 & -4.1333940000 & 2.3007090000 \\
\hline $\mathrm{C}$ & -4.8883670000 & 5.4689080000 & 2.0441250000 \\
\hline $\mathrm{C}$ & -0.9220590000 & 4.2838390000 & -2.2266440000 \\
\hline $\mathrm{C}$ & -6.4045260000 & -4.0301360000 & -1.1448940000 \\
\hline $\mathrm{C}$ & -2.6377490000 & -3.7670860000 & 3.6141710000 \\
\hline $\mathrm{C}$ & 4.7606330000 & -5.3224440000 & -2.0734160000 \\
\hline $\mathrm{C}$ & 70000 & 4.1345900000 & 80000 \\
\hline $\mathrm{C}$ & 2.6401780000 & 3.9052910000 & -3.6298220000 \\
\hline $\mathrm{C}$ & -1.0252580000 & 1.8763950000 & -1.8203850000 \\
\hline $\mathrm{C}$ & -2.8923340000 & -2.3907140000 & 1.6259610000 \\
\hline $\mathrm{C}$ & -4.3059330000 & 3.4365900000 & 0.7796000000 \\
\hline $\mathrm{C}$ & -6.209 & 10000 & 390000 \\
\hline $\mathrm{C}$ & -1.5942830000 & 0.9825470000 & 0.4292970000 \\
\hline $\mathrm{C}$ & -1.5181260000 & 0.7675720000 & -0.9517260000 \\
\hline $\mathrm{C}$ & -1.9360450000 & -0.4634710000 & -1.4837260000 \\
\hline $\mathrm{C}$ & -2.3357 & -1.4590340000 & -0.5869790000 \\
\hline $\mathrm{C}$ & -2.3847 & -1.2525060000 & 0.7901380000 \\
\hline $\mathrm{C}$ & -2.0636050000 & 0.0142220000 & 1.3144730000 \\
\hline $\mathrm{C}$ & -2.2464080000 & 0.4055830000 & 2.7614250000 \\
\hline $\mathrm{C}$ & -5.0847800000 & 1.2077370000 & 2.7057140000 \\
\hline $\mathrm{C}$ & -4.6392520000 & 1.8156750000 & -1.0448550000 \\
\hline $\mathrm{C}$ & -4.7371980000 & 2.0817000000 & 0.3277310000 \\
\hline $\mathrm{C}$ & -5.1791290000 & 1.0783430000 & 1.2046430000 \\
\hline $\mathrm{C}$ & -5.6346840000 & -0.1232850000 & 0.6442870000 \\
\hline $\mathrm{C}$ & -5.6055310000 & -0.3588650000 & -0.7275980000 \\
\hline $\mathrm{C}$ & -5.0436370000 & 0.6028170000 & -1.5916000000 \\
\hline $\mathrm{C}$ & -4.8494500000 & 0.3461310000 & -3.0644090000 \\
\hline $\mathrm{C}$ & -2.0804750000 & -0.7089820000 & -2.9651250000 \\
\hline $\mathrm{C}$ & 4.3303870000 & -3.3596080000 & -0.6779480000 \\
\hline $\mathrm{C}$ & 6.3145600000 & 1.7285190000 & 1.2247090000 \\
\hline $\mathrm{C}$ & 1.0452270000 & -1.7391530000 & 1.8183760000 \\
\hline $\mathrm{C}$ & 2.9620590000 & 2.4305590000 & -1.7173220000 \\
\hline $\mathrm{C}$ & 4.7478930000 & -1.7134260000 & 1.1107100000 \\
\hline $\mathrm{C}$ & 4.7670600000 & -1.9954180000 & -0.2586160000 \\
\hline $\mathrm{C}$ & 5.1309530000 & -0.9914120000 & -1.1720130000 \\
\hline $\mathrm{C}$ & 5.6297000000 & 0.2084350000 & -0.6534820000 \\
\hline $\mathrm{C}$ & 5.6907980000 & 0.4589930000 & 0.7172440000 \\
\hline $\mathrm{C}$ & 5.1711210000 & -0.4864310000 & 1.6188200000 \\
\hline $\mathrm{C}$ & 5.0077450000 & -0.1832850000 & 3.0850970000 \\
\hline
\end{tabular}




\begin{tabular}{|c|c|c|c|}
\hline $\mathrm{C}$ & 2.2180840000 & 0.7902180000 & 2.9160570000 \\
\hline & 1.5506500000 & -0.8782830000 & -0.4612690000 \\
\hline$C$ & 1.5567260000 & -0.6610860000 & 0.9180330000 \\
\hline $\mathrm{C}$ & 2.0551710000 & 0.5509180000 & 1.4330200000 \\
\hline $\mathrm{C}$ & 2.4737790000 & 1.5215140000 & 0.5228000000 \\
\hline $\mathrm{C}$ & 2.4407910000 & 1.3158350000 & -0.8594950000 \\
\hline $\mathrm{C}$ & 2.0135670000 & 0.0770360000 & -1.3694540000 \\
\hline $\mathrm{C}$ & 2.1025260000 & -0.2810970000 & -2.8309370000 \\
\hline $\mathrm{C}$ & 4.9233730000 & -1.0987740000 & -2.6681140000 \\
\hline $\mathrm{H}$ & 1.1288130000 & -0.5910650000 & -3.2150770000 \\
\hline $\mathrm{H}$ & 2.4433870000 & 0.5520990000 & -3.4409780000 \\
\hline $\mathrm{H}$ & 1.5882740000 & 1.6219230000 & 3.2450910000 \\
\hline $\mathrm{H}$ & 1.9288070000 & -0.0802070000 & 3.5005510000 \\
\hline $\mathrm{H}$ & 4.5765100000 & -1.0347360000 & 3.6164340000 \\
\hline $\mathrm{H}$ & 5.9645900000 & 0.0901560000 & 0000 \\
\hline $\mathrm{H}$ & 5.0652330000 & -0.1182150000 & -3.1298990000 \\
\hline $\mathrm{H}$ & 5.6179760000 & -1.7885240000 & -3.1544290000 \\
\hline $\mathrm{H}$ & 4.3687600000 & -2.4714880000 & 1.7896250000 \\
\hline $\mathrm{H}$ & 5.9445600000 & 0.9877870000 & -1.3398270000 \\
\hline $\mathrm{H}$ & 460000 & 2.4471700000 & 0000 \\
\hline $\mathrm{H}$ & 1.2063000000 & -1.8328260000 & -0.8456850000 \\
\hline $\mathrm{H}$ & -1.5177140000 & -0.0953890000 & 3.4012880000 \\
\hline $\mathrm{H}$ & -2.1051950000 & 1.4837040000 & 2.8752020000 \\
\hline $\mathrm{H}$ & -4.7714810000 & 2.2036320000 & 3.0179470000 \\
\hline $\mathrm{H}$ & -6.0411210000 & 0.9825 & 0000 \\
\hline $\mathrm{H}$ & -5.9842480000 & -0.9093920000 & 1.3060210000 \\
\hline $\mathrm{H}$ & -4.2209060000 & 2.5789500000 & 650000 \\
\hline $\mathrm{H}$ & -5.8064050000 & 0.1246050000 & -3.5399250000 \\
\hline $\mathrm{H}$ & -4.3848470000 & 1.2068440000 & -3.5514890000 \\
\hline $\mathrm{H}$ & -1.7506170000 & 0.1431910000 & 940000 \\
\hline $\mathrm{H}$ & -1.4935270000 & -1.5777600000 & -3.2776740000 \\
\hline $\mathrm{H}$ & -1.3076580000 & 1.9496960000 & 100000 \\
\hline $\mathrm{H}$ & -2.6886510000 & -2.4076510000 & -0.9788490000 \\
\hline $\mathrm{H}$ & -5.3833050000 & 5.6171650000 & 3.0053970000 \\
\hline $\mathrm{H}$ & -5.2284970000 & 6.2348000000 & 1.3399190000 \\
\hline $\mathrm{H}$ & -3.8103080000 & 5.5728670000 & 2.1696250000 \\
\hline $\mathrm{H}$ & -5.9813920000 & -4.8281850000 & -0.5336390000 \\
\hline $\mathrm{H}$ & -6.1375420000 & -4.1899830000 & -2.1948350000 \\
\hline $\mathrm{H}$ & -7.4959160000 & -4.0548390000 & -1.0710870000 \\
\hline $\mathrm{H}$ & -1.8925260000 & -3.8980080000 & 4.3998950000 \\
\hline $\mathrm{H}$ & -2.7854200000 & -4.7148090000 & 3.0880810000 \\
\hline $\mathrm{H}$ & -3.5929000000 & -3.4786020000 & 4.0635860000 \\
\hline $\mathrm{H}$ & 1.5729410000 & -4.9894050000 & 2.0495390000 \\
\hline $\mathrm{H}$ & -0.0970980000 & -4.3732310000 & 2.0731690000 \\
\hline $\mathrm{H}$ & 1.0248170000 & -3.9203020000 & 3.3706330000 \\
\hline $\mathrm{H}$ & -1.5342850000 & 5.1395090000 & -1.9405480000 \\
\hline $\mathrm{H}$ & -1.0171760000 & 4.1065120000 & -3.3015270000 \\
\hline
\end{tabular}




$\begin{array}{lrrr}\mathrm{H} & 0.1270620000 & 4.5057310000 & -2.0057070000 \\ \mathrm{H} & 1.8858090000 & 4.0447920000 & -4.4050020000 \\ \mathrm{H} & 3.6000900000 & 3.6671450000 & -4.0964830000 \\ \mathrm{H} & 2.7664860000 & 4.8377260000 & -3.0708760000 \\ \mathrm{H} & 6.2857480000 & 4.3384890000 & 2.0293820000 \\ \mathrm{H} & 6.1175640000 & 4.9150810000 & 0.3467270000 \\ \mathrm{H} & 7.6272130000 & 4.1404700000 & 0.8954510000 \\ \mathrm{H} & 5.4963570000 & -5.6223000000 & -2.8200600000 \\ \mathrm{H} & 3.7735610000 & -5.2637830000 & -2.5389440000 \\ \mathrm{H} & 4.7217280000 & -6.0731190000 & -1.2793640000 \\ \mathrm{H} & -2.0401740000 & 3.2970160000 & -0.7109780000 \\ \mathrm{H} & -1.2731850000 & -2.2771570000 & 2.8828480000 \\ \mathrm{H} & -6.0886980000 & 3.7345730000 & 1.7142530000 \\ \mathrm{H} & -5.1611960000 & -2.7768350000 & 0.0609100000 \\ \mathrm{H} & 6.0129840000 & -3.6086160000 & -1.8034480000 \\ \mathrm{H} & 5.2704900000 & 2.8471290000 & -0.1638810000 \\ \mathrm{H} & 2.0882680000 & -3.1917110000 & 0.7784450000 \\ \mathrm{H} & 1.2788640000 & 2.4107110000 & -2.8986010000\end{array}$

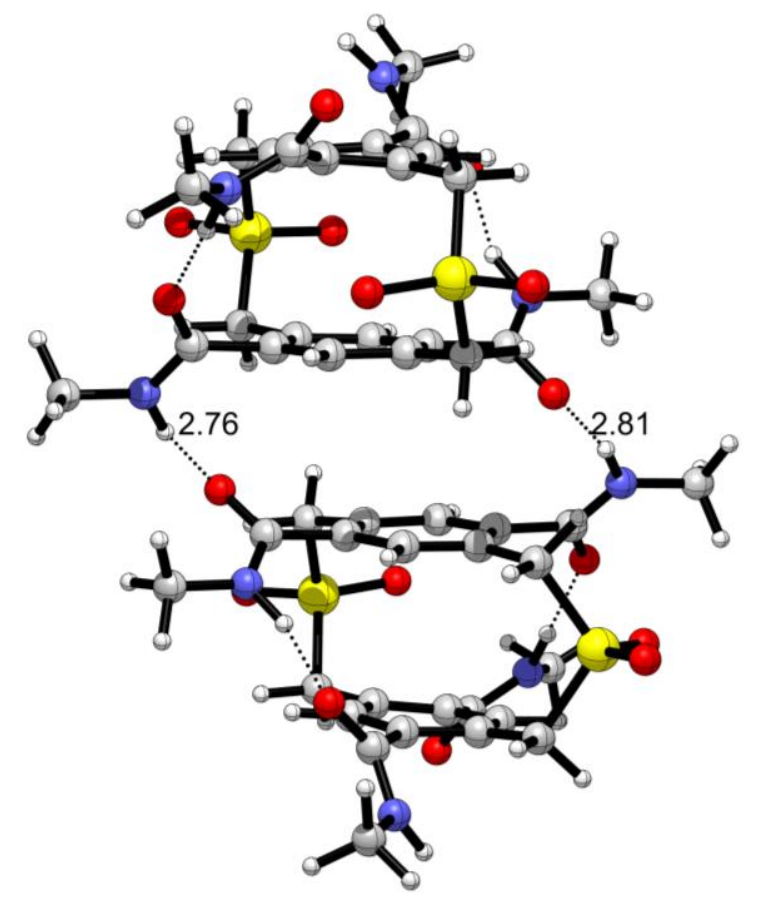

Heterochiral 2 dimer $S_{\mathrm{p}}$ (top) $\cdots R_{\mathrm{p}}$ (bottom)

Energy: -4493.804219

$\begin{array}{lrrr}\mathrm{S} & 3.3239640000 & 3.4335360000 & 1.3070760000 \\ \mathrm{~S} & 3.8364000000 & -3.2853210000 & -1.6788920000 \\ \mathrm{~S} & -3.8986300000 & -2.6508540000 & -2.4086910000 \\ \mathrm{~S} & -3.7919400000 & 3.0981800000 & 1.9916270000 \\ \mathrm{O} & 3.5211880000 & 4.5413260000 & 2.4846950000 \\ \mathrm{O} & 2.7400520000 & 3.8924110000 & -0.1483290000 \\ \mathrm{O} & 3.7076340000 & -4.4012400000 & -2.8570630000\end{array}$




\begin{tabular}{|c|c|c|c|}
\hline ? & 173380000 & -3.7473100000 & 000 \\
\hline 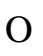 & -3.6668710000 & -4.1275440000 & -3.0574920000 \\
\hline & -4.5881610000 & -1.4727220000 & 000 \\
\hline & -3.7348400000 & 4.1219450000 & 3.2565390000 \\
\hline & -4.2080620000 & 3.6401740000 & 000 \\
\hline & -0.2131500000 & 0.6252180000 & 3.1950030000 \\
\hline & -3.9464430000 & 1.4515780000 & -2.8669370000 \\
\hline & -3.0996710000 & -3.2729280000 & 960000 \\
\hline & -7.2123670000 & 2.3787530000 & 420000 \\
\hline & 3.5158900000 & 7840000 & 90000 \\
\hline & 940000 & 9080000 & 00000 \\
\hline & 0.25 & -0.7 & \\
\hline & 730000 & -1.1 & 0000 \\
\hline & 220000 & -1.3 & 40000 \\
\hline & -2.2108680000 & & 70000 \\
\hline & 720000 & -4.1 & 390000 \\
\hline & -6.16 & & 90000 \\
\hline & 5.28 & 0000 & 0000 \\
\hline & 40000 & -2.3 & 50000 \\
\hline & 1.41 & & 0000 \\
\hline & 2.29 & -0.2 & 00 \\
\hline & 0.92 & & 000 \\
\hline & 10000 & -5.4 & 50000 \\
\hline & -0.74 & -1.7 & 0000 \\
\hline & -6.77 & 00 & 0000 \\
\hline & -2.72 & 0.4 & 0000 \\
\hline C & 4.9 & & 000 \\
\hline $\mathrm{C}$ & 50000 & -3.5 & 20000 \\
\hline C & 80000 & -0.4 & 0000 \\
\hline & -0.95 & -0.2 & 20000 \\
\hline $\mathrm{C}$ & -2.91 & 00 & 90000 \\
\hline C & -4.23 & -3.1 & 30000 \\
\hline $\mathrm{C}$ & -6.40 & 00 & 30000 \\
\hline $\mathrm{C}$ & -1.5 & -1.0 & 70000 \\
\hline & -1.50 & 0.0 & 60000 \\
\hline$c$ & -1.92 & 00 & 0000 \\
\hline$c$ & 40000 & & 50000 \\
\hline $\mathrm{C}$ & -2.40 & 0.3 & 180000 \\
\hline $\mathrm{C}$ & -2.06 & -0.9 & 970000 \\
\hline $\mathrm{C}$ & -2.16 & -2.1 & 120000 \\
\hline $\mathrm{C}$ & 10000 & -2.9 & 830000 \\
\hline $\mathrm{C}$ & -4.61 & 4500000 & 180000 \\
\hline $\mathrm{C}$ & 50000 & 2010000 & 1.3127750000 \\
\hline $\mathrm{C}$ & 80000 & 1730000 & -0.0088540000 \\
\hline$c$ & -5.66 & -0.5 & 330000 \\
\hline C & -5.6691050000 & 0.5953280000 & 0.3541700000 \\
\hline $\mathrm{C}$ & -5.0909590000 & 0.5276690000 & 1.6372810000 \\
\hline
\end{tabular}




\begin{tabular}{|c|c|c|c|}
\hline $\mathrm{C}$ & -5.0091320000 & 1.7227490000 & 60000 \\
\hline & -2.0168770000 & 2.4295630000 & 1.8047840000 \\
\hline & 4.4812880000 & 2.8675840000 & -1.9888950000 \\
\hline $\mathrm{C}$ & 6.4724590000 & -2.0355010000 & 0.3518130000 \\
\hline $\mathrm{C}$ & 1.0346660000 & 0.1223400000 & -2.4583910000 \\
\hline $\mathrm{C}$ & 3.0463450000 & -0.5849870000 & 2.9394370000 \\
\hline $\mathrm{C}$ & 4.8021970000 & 0.4325420000 & -1.9915590000 \\
\hline C & 4.8453960000 & 1.6160800000 & -1.2501610000 \\
\hline $\mathrm{C}$ & 5.1996070000 & 1.5554580000 & 0.1122340000 \\
\hline $\mathrm{C}$ & 5.6732550000 & 0.3373850000 & 0.6181420000 \\
\hline $\mathrm{C}$ & 5.7530950000 & -0.8153470000 & -0.1630310000 \\
\hline $\mathrm{C}$ & 5.2316630000 & -0.7873770000 & -1.4695420000 \\
\hline $\mathrm{C}$ & 5.1321590000 & -2.0226240000 & -2.3032740000 \\
\hline $\mathrm{C}$ & 2.1118110000 & -2.4933720000 & -1.5570630000 \\
\hline $\mathrm{C}$ & 1.5737850000 & 1.0224260000 & 20000 \\
\hline $\mathrm{C}$ & 1.5677840000 & -0.0641490000 & -1.0718160000 \\
\hline $\mathrm{C}$ & 2.0318020000 & -1.3175340000 & 8610000 \\
\hline $\mathrm{C}$ & 2.4673150000 & -1.4444380000 & 0.6929360000 \\
\hline $\mathrm{C}$ & 2.4818890000 & -0.3551440000 & 1.5657190000 \\
\hline $\mathrm{C}$ & 2.0513180000 & 0.903 & 30000 \\
\hline $\mathrm{C}$ & 2.1302000000 & 2.1199960000 & 950000 \\
\hline $\mathrm{C}$ & 5.0770900000 & 2.7003210000 & 520000 \\
\hline H & 1.1941480000 & 2.6785360000 & 2.0226910000 \\
\hline $\mathrm{H}$ & 2.4850820000 & 1.9410620000 & 2.9907830000 \\
\hline $\mathrm{H}$ & 1.539 & -3.354 & 30000 \\
\hline $\mathrm{H}$ & 1.8223100000 & -2.2798730000 & -2.5833640000 \\
\hline $\mathrm{H}$ & 4.8201360000 & -1.8398870000 & -3.3319090000 \\
\hline $\mathrm{H}$ & 6.0439890000 & -2.6236630000 & -2.2581720000 \\
\hline H & 5.3592330000 & 2.4214940000 & 2.0879350000 \\
\hline $\mathrm{H}$ & 5.6091970000 & 3.6126550000 & 10000 \\
\hline $\mathrm{H}$ & 4.4358010000 & 0.4852660000 & -3.0121480000 \\
\hline $\mathrm{H}$ & 5.9938220000 & 0.2815220000 & 1.6527640000 \\
\hline $\mathrm{H}$ & 2.8672240000 & -2.3970000000 & 1.0256410000 \\
\hline H & 1.2393940000 & 1.9933230000 & -0.5461790000 \\
\hline $\mathrm{H}$ & -1.6088040000 & -2.0766830000 & -2.7250670000 \\
\hline $\mathrm{H}$ & -1.8495780000 & -3.0323680000 & -1.2518830000 \\
\hline $\mathrm{H}$ & -4.7313040000 & -3.8563040000 & -0.5062530000 \\
\hline $\mathrm{H}$ & -6.0141780000 & -3.1094210000 & -1.4972140000 \\
\hline $\mathrm{H}$ & -6.0369220000 & -0.4578860000 & -1.4785520000 \\
\hline $\mathrm{H}$ & -4.1934790000 & -0.7759680000 & 3.0931210000 \\
\hline $\mathrm{H}$ & -5.9481220000 & 2.2836330000 & 2.5372960000 \\
\hline $\mathrm{H}$ & -4.6763380000 & 1.4933470000 & 3.5450540000 \\
\hline $\mathrm{H}$ & -1.6827560000 & 2.2129620000 & 2.8163400000 \\
\hline $\mathrm{H}$ & -1.5194980000 & 3.3316570000 & 1.4398670000 \\
\hline $\mathrm{H}$ & -1.2932170000 & -2.0535640000 & 0.6904870000 \\
\hline $\mathrm{H}$ & -2.6991930000 & 2.4264670000 & -0.7877610000 \\
\hline $\mathrm{H}$ & -5.4217350000 & -6.2296940000 & 1.9945270000 \\
\hline
\end{tabular}




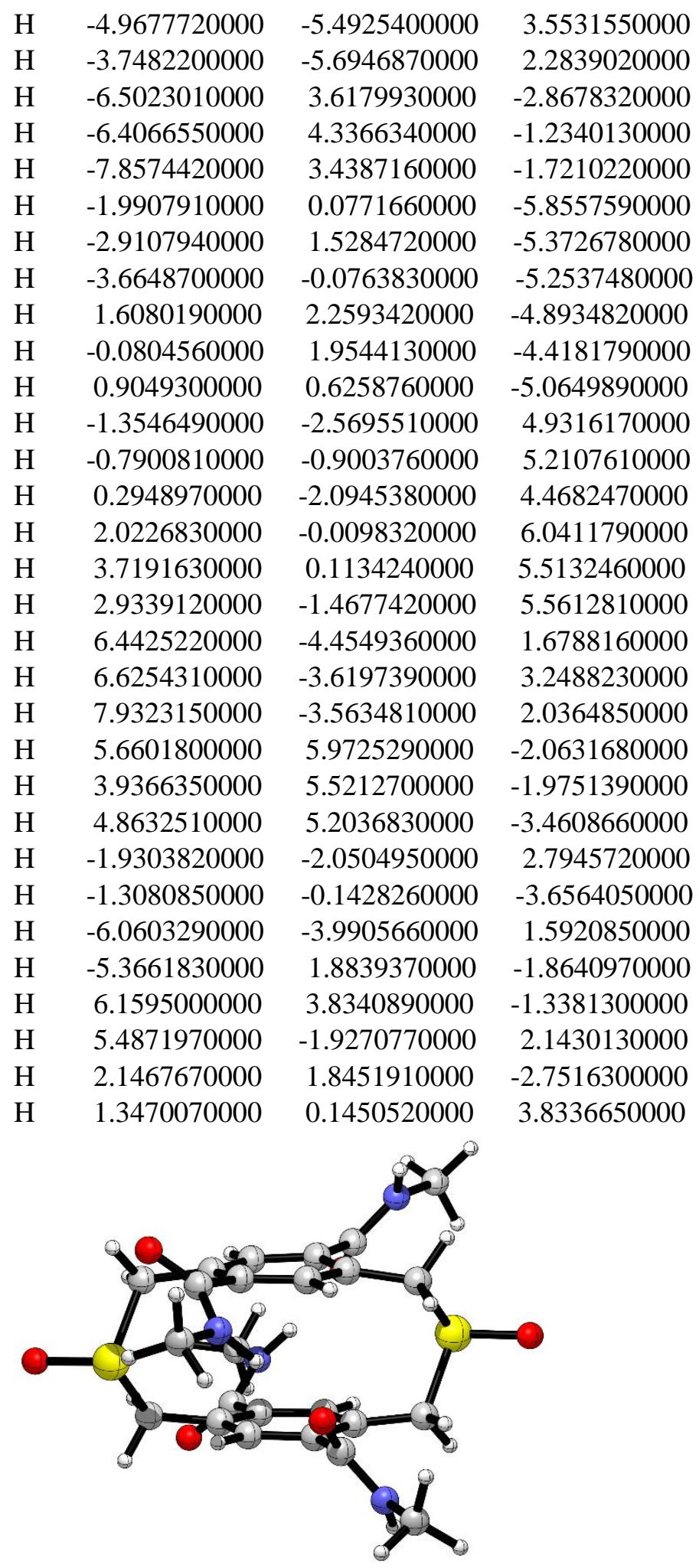

$\left(S_{\mathrm{p}}\right)$-dt[3.3]pCpTA-SO chair anti- $S=O$ away Energy: -2397.02932144

$\begin{array}{rrrr}\text { S } & -3.1380960000 & 1.7364110000 & 0.0007510000 \\ \text { S } & 3.2703760000 & -1.8474950000 & -0.0357400000\end{array}$ 


\begin{tabular}{|c|c|c|c|}
\hline 9 & -4.0287650000 & 1471040000 & 000 \\
\hline C & 4.2113050000 & -3.2226020000 & -01173590000 \\
\hline & 1.7323720000 & 880000 & 10000 \\
\hline & -3.1233040000 & -1.7810800000 & 0.6947160000 \\
\hline & -0.1105350000 & -3.5130890000 & -2.8375890000 \\
\hline & 3.5814480000 & -0.0866700000 & 2.7893770000 \\
\hline & 3.2703760000 & 1.6890720000 & 1.3830410000 \\
\hline & -1.8240170000 & -3.1802200000 & -1.3593140000 \\
\hline & 0.5373080000 & 3.6191270000 & 38160000 \\
\hline & -3.5505360000 & -0.47 & 0000 \\
\hline & 4.6406300000 & 2.1500750000 & 0000 \\
\hline & -2.3459070000 & -4.5263290000 & -1.5401890000 \\
\hline & 0.9683130000 & 5.0123200000 & 40000 \\
\hline & 0.9469460000 & 330000 & 0000 \\
\hline C & -0.7 & -2.76 & 000 \\
\hline & 000 & 10000 & 0000 \\
\hline C & -4.96 & -0.8 & 0000 \\
\hline $\mathrm{C}$ & 540000 & -0.96 & 60000 \\
\hline & -2.00 & 2.04 & 70000 \\
\hline $\mathrm{C}$ & -1.9 & & 000 \\
\hline & 000 & 0000 & 0000 \\
\hline $\mathrm{C}$ & 000 & 0000 & 0000 \\
\hline $\mathrm{C}$ & -0.93 & 0.86 & 000 \\
\hline $\mathrm{C}$ & -1.29 & -0.50 & 0000 \\
\hline $\mathrm{C}$ & -0.2 & -1.4 & 000 \\
\hline $\mathrm{C}$ & 000 & 0000 & 0000 \\
\hline $\mathrm{C}$ & 2.08 & -2.2 & 000 \\
\hline C & 80000 & -2.08 & 0000 \\
\hline $\mathrm{C}$ & 600000 & 0000 & 000 \\
\hline $\mathrm{C}$ & -0.92 & 1.01 & 0000 \\
\hline $\mathrm{C}$ & $-1.26^{\prime}$ & -0.33 & 0000 \\
\hline $\mathrm{C}$ & -0.3 & -1.3 & 000 \\
\hline $\mathrm{C}$ & 50000 & -1.02 & 0000 \\
\hline . & 1.40 & 0.32 & 000 \\
\hline 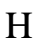 & 4.76 & 0000 & 000 \\
\hline $\mathrm{H}$ & 000 & 80000 & 0000 \\
\hline $\mathrm{H}$ & 4.85 & 2.3 & 000 \\
\hline $\mathrm{H}$ & 90000 & 2.16 & 0000 \\
\hline $\mathrm{H}$ & -5.47 & -0.21 & 0000 \\
\hline $\mathrm{H}$ & -5.1225110000 & -1.8715400000 & 40000 \\
\hline $\mathrm{H}$ & -5.40 & 60000 & 40000 \\
\hline $\mathrm{H}$ & -3.1721130000 & 90000 & 20000 \\
\hline $\mathrm{H}$ & 0.2678490000 & 620000 & 80000 \\
\hline $\mathrm{H}$ & 1.9745850000 & 5.1179700000 & -2.8237470000 \\
\hline $\mathrm{H}$ & 490000 & 5.3838340000 & -1.3813080000 \\
\hline 11 & -3.1417090000 & -4.6869610000 & -0.8121000000 \\
\hline $\mathrm{H}$ & -1.5569940000 & -5.2698830000 & -1.3933420000 \\
\hline
\end{tabular}




$\begin{array}{rrrc}\mathrm{H} & -2.7450300000 & -4.6611600000 & -2.5501720000 \\ \mathrm{H} & -0.0012280000 & 3.2768980000 & -3.1887870000 \\ \mathrm{H} & -2.2646360000 & -2.5837250000 & -0.6571450000 \\ \mathrm{H} & 1.6601520000 & -3.2025040000 & 1.2110350000 \\ \mathrm{H} & 2.7931690000 & -2.2804070000 & 2.2546370000 \\ \mathrm{H} & -1.4142500000 & 2.9331130000 & 1.2497040000 \\ \mathrm{H} & -2.6152940000 & 2.1426660000 & 2.2752020000 \\ \mathrm{H} & 2.8117260000 & -2.0751260000 & -2.3642700000 \\ \mathrm{H} & 1.7143550000 & -3.0989120000 & -1.4522660000 \\ \mathrm{H} & -1.6525580000 & 3.0655820000 & -1.3732440000 \\ \mathrm{H} & -2.7310480000 & 2.0267800000 & -2.3255690000 \\ \mathrm{H} & 0.7104400000 & 2.2189820000 & 1.8385140000 \\ \mathrm{H} & -0.5941620000 & -2.5124190000 & 1.4147910000 \\ \mathrm{H} & -2.3150390000 & -0.6003520000 & -1.9060500000 \\ \mathrm{H} & 2.4439140000 & 0.5942350000 & -1.4292410000\end{array}$

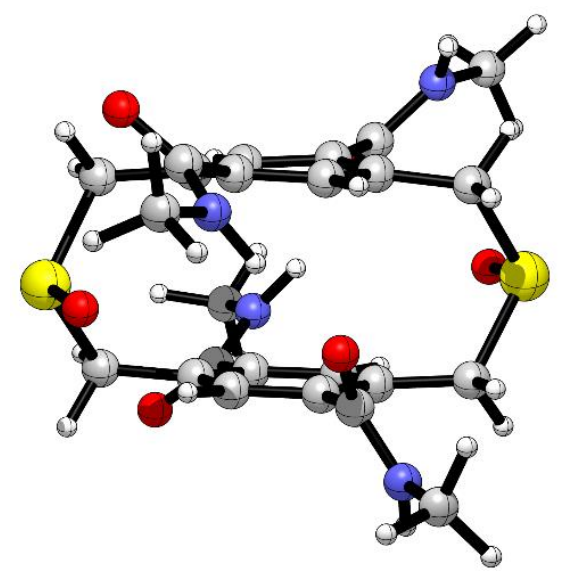

$\left(S_{\mathrm{p}}\right)$-dtt3.3]pCpTA-SO chair anti-

$S=O$ towards

Energy: -2397.02932144

$\begin{array}{lrrr}\mathrm{S} & -2.0666640000 & 3.0549280000 & 0.0040510000 \\ \mathrm{~S} & 2.1769700000 & -3.1547340000 & -0.2113660000 \\ \mathrm{O} & -3.2791900000 & 1.9219770000 & -0.2197790000 \\ \mathrm{O} & 3.3379810000 & -1.9430800000 & -0.2030420000 \\ \mathrm{O} & 2.8157090000 & 2.2089290000 & -0.4115630000 \\ \mathrm{O} & -3.6379640000 & -0.7544910000 & 0.9033860000 \\ \mathrm{O} & -1.5586390000 & -3.0352530000 & -2.9395910000 \\ \mathrm{O} & 3.1435800000 & -1.4805990000 & 2.9192010000 \\ \mathrm{~N} & 3.6502810000 & 0.3671220000 & 1.6632630000 \\ \mathrm{~N} & -2.9778470000 & -2.0524850000 & -1.4429070000 \\ \mathrm{~N} & 2.1419250000 & 3.0350260000 & -2.4186430000 \\ \mathrm{~N} & -3.4927020000 & 0.7683940000 & 2.5793380000 \\ \mathrm{C} & 5.0776070000 & 0.2169800000 & 1.8936800000 \\ \mathrm{C} & -4.0138280000 & -3.0503240000 & -1.6582710000 \\ \mathrm{C} & 3.1460680000 & 4.0913850000 & -2.4226850000 \\ \mathrm{C} & 2.0516080000 & 2.1387200000 & -1.4036210000\end{array}$




\begin{tabular}{|c|c|c|c|}
\hline $\mathrm{C}$ & -1.8191360000 & -2.1127180000 & -2.12 \\
\hline & 2.7981230000 & -0.5604350000 & 2.1406250000 \\
\hline C & -4.9325900000 & 0.9823780000 & 2.6080700000 \\
\hline C & -2.9328420000 & -0.0115730000 & 1.6264840000 \\
\hline $\mathrm{C}$ & -0.8764130000 & 2.7665120000 & -1.4705940000 \\
\hline $\mathrm{C}$ & -1.0728200000 & 2.5147770000 & 1.5615630000 \\
\hline C & 1.3493860000 & -0.3910000000 & 1.7715430000 \\
\hline $\mathrm{C}$ & 0.7796390000 & 0.8821790000 & 1.7645600000 \\
\hline $\mathrm{C}$ & -0.5993490000 & 1.0927940000 & 1.6378560000 \\
\hline $\mathrm{C}$ & -1.4372300000 & -0.0389000000 & 1.5702180000 \\
\hline $\mathrm{C}$ & -0.8544730000 & -1.3025810000 & 1.4432390000 \\
\hline $\mathrm{C}$ & 0.5238900000 & -1.5038150000 & 1.5224580000 \\
\hline $\mathrm{C}$ & 1.0798270000 & -2.8788390000 & 1.3444850000 \\
\hline $\mathrm{C}$ & 1.0497920000 & -2.7174420000 & -1.6956800000 \\
\hline $\mathrm{C}$ & 1.0098810000 & 1.0754930000 & -1.5589920000 \\
\hline $\mathrm{C}$ & -0.3630790000 & 1.3694160000 & -1.6298900000 \\
\hline $\mathrm{C}$ & -1.2641760000 & 0.3122480000 & -1.8139370000 \\
\hline $\mathrm{C}$ & -0.8274950000 & -1.0134780000 & -1.8674270000 \\
\hline C & 0.5430200000 & -1.3055470000 & -1.7315690000 \\
\hline $\mathrm{C}$ & 870000 & 30000 & -1.6039070000 \\
\hline $\mathrm{H}$ & 5.5902850000 & 1.0927870000 & 1.4930310000 \\
\hline $\mathrm{H}$ & 5.4486390000 & -0.6876990000 & 1.4003400000 \\
\hline $\mathrm{H}$ & 5.2811150000 & 0.1319900000 & 2.9636350000 \\
\hline $\mathrm{H}$ & 3.3488430000 & 0.9776280000 & 0.9074 \\
\hline $\mathrm{H}$ & -5.1673070000 & 010000 & 3.3844250000 \\
\hline $\mathrm{H}$ & -5.4652310000 & 0.0498240000 & 2.8130840000 \\
\hline $\mathrm{H}$ & -5.2510100000 & 1.3644240000 & 1.6348760000 \\
\hline $\mathrm{H}$ & -2.9119580000 & 1.1865380000 & 3.2858120000 \\
\hline $\mathrm{H}$ & 2.8539330000 & 4.8602430000 & -3.1390520000 \\
\hline $\mathrm{H}$ & 4.1330830000 & 3.7014790000 & -2.6906070000 \\
\hline $\mathrm{H}$ & 3.2156530000 & 4.5294180000 & -1.4256330000 \\
\hline $\mathrm{H}$ & -4.7978770000 & -2.8891110000 & -0.9179790000 \\
\hline $\mathrm{H}$ & -3.6080360000 & -4.0612880000 & -1.5525190000 \\
\hline $\mathrm{H}$ & -4.4376190000 & -2.9657890000 & -2.6636350000 \\
\hline $\mathrm{H}$ & 1.5931600000 & 2.8770450000 & -3.2489160000 \\
\hline $\mathrm{H}$ & -3.1272850000 & -1.3914280000 & -0.6750090000 \\
\hline $\mathrm{H}$ & 0.2999040000 & -3.6389300000 & 1.2615830000 \\
\hline $\mathrm{H}$ & 1.7844010000 & -3.1206130000 & 2.1423720000 \\
\hline $\mathrm{H}$ & -0.2312910000 & 3.2115650000 & 1.5898610000 \\
\hline $\mathrm{H}$ & -1.7811330000 & 2.7954720000 & 2.3423080000 \\
\hline $\mathrm{H}$ & 1.7227910000 & -2.9225430000 & -2.5312560000 \\
\hline $\mathrm{H}$ & 0.2546180000 & -3.4604810000 & -1.7135620000 \\
\hline $\mathrm{H}$ & -0.0909510000 & 3.5134010000 & -1.3486740000 \\
\hline $\mathrm{H}$ & -1.5276540000 & 3.0633990000 & -2.2967520000 \\
\hline $\mathrm{H}$ & 1.4356940000 & 1.7398770000 & 1.8657780000 \\
\hline 1. & -1.5152470000 & -2.1502500000 & 1.2897660000 \\
\hline $\mathrm{H}$ & -2.3248840000 & 0.5307400000 & -1.8646330000 \\
\hline
\end{tabular}



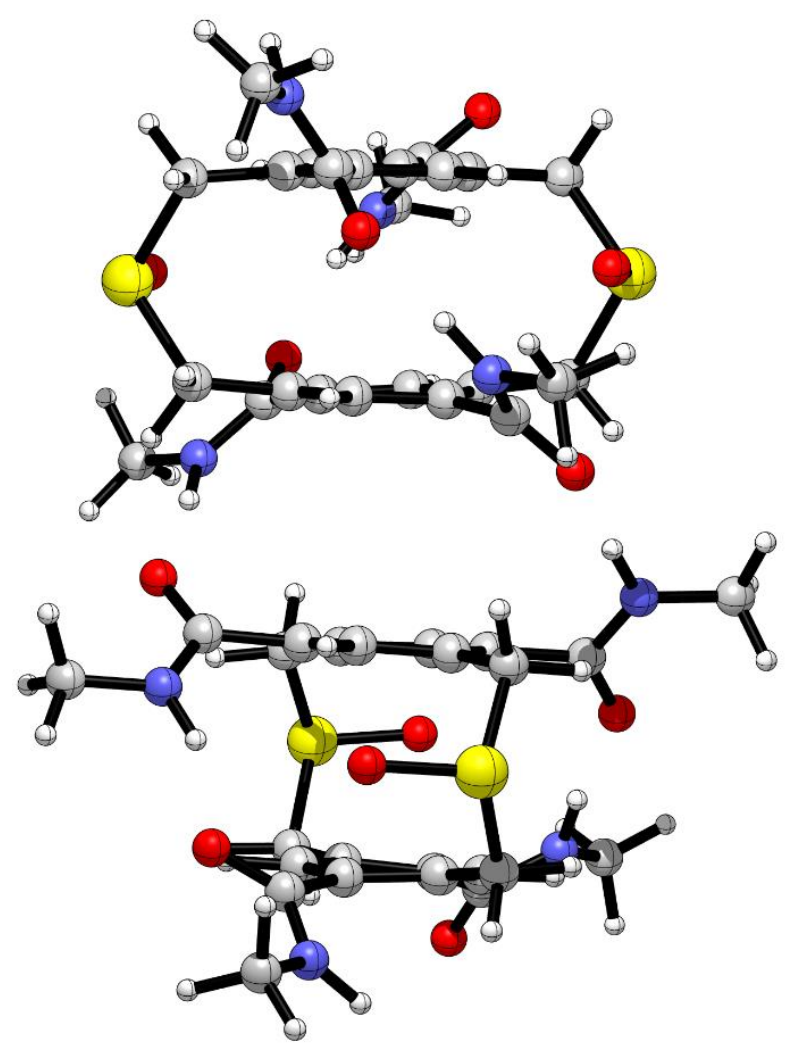

$\left(S_{\mathrm{p}}\right)$-6-2 chair anti- dimer $S=O$ towards

Energy: -4794.13724428

S $\quad 3.6713850000 \quad-3.4253290000$

S $\quad 3.2944070000 \quad 3.5584690000 \quad-1.4036030000$

S $\quad-3.8173410000 \quad-0.1189510000 \quad-3.6646370000$

$\begin{array}{llll}\mathrm{S} & -3.5459440000 & 0.1543670000 & 3.8486240000\end{array}$

$\begin{array}{llll}\text { O } & 3.4849650000 & -3.7490340000 & -0.3134320000\end{array}$

$\begin{array}{llll}\text { O } & 3.2707290000 & 3.8467940000 & 0.2462620000\end{array}$

$\begin{array}{llll}\text { O } & -3.9984980000 & 1.5310760000 & -3.4482390000\end{array}$

O $\quad-3.5776740000 \quad-1.5011950000 \quad 3.5721730000$

$\begin{array}{llll}\text { O } & -6.2909310000 & 3.1577520000 & 1.7200080000\end{array}$

$\begin{array}{llll}\text { O } & -2.6399440000 & 3.3536390000 & -1.5477850000\end{array}$

$\begin{array}{llll}\text { O } & -4.2101030000 & -3.5823970000 & -0.0275370000\end{array}$

$\begin{array}{llll}\text { O } & -0.6057590000 & -1.8628850000 & 2.8887770000\end{array}$

$\begin{array}{llll}\mathrm{O} & 0.6447160000 & 0.2169120000 & -3.4477800000\end{array}$

$\begin{array}{llll}\mathrm{O} & 4.6156290000 & -2.5326240000 & -2.6849890000\end{array}$

$\begin{array}{llll}\text { O } & 6.4417120000 & 3.4999450000 & 0.5570440000\end{array}$

$\begin{array}{llll}\mathrm{O} & 3.0418480000 & 1.2681770000 & 3.3244130000\end{array}$

$\mathrm{N} \quad-6.2314210000 \quad-3.4051230000 \quad-1.0436320000$

$\begin{array}{llll}\mathrm{N} & -1.1496470000 & 2.2632350000 & -2.8774460000\end{array}$

$\begin{array}{llll}\mathrm{N} & -4.8656630000 & 3.6703590000 & 0.0087040000\end{array}$

$\mathrm{N} \quad-2.0490790000 \quad-3.2008000000 \quad 1.7564420000$

$\begin{array}{llll}\mathrm{N} & 1.2952430000 & -0.1248540000 & 3.7427100000\end{array}$ 


\begin{tabular}{|c|c|c|c|}
\hline N & 6.2757940000 & -3.2404470000 & -1.3114 \\
\hline & 5.1379820000 & 2.8927730000 & 2.3423120000 \\
\hline v & 2.2518720000 & -1.3812930000 & -3.3598410000 \\
\hline & -6.3211550000 & -4.8367610000 & -1.3025510000 \\
\hline C & -1.1697340000 & 3.3093220000 & -3.8866790000 \\
\hline $\mathrm{C}$ & -1.9841440000 & 2.3155950000 & -1.8308380000 \\
\hline $\mathrm{C}$ & -2.2367510000 & -0.6868760000 & -2.7385580000 \\
\hline $\mathrm{C}$ & -5.2570130000 & -0.8632090000 & -2.6425690000 \\
\hline $\mathrm{C}$ & -5.1673480000 & -2.8614620000 & -0.4045650000 \\
\hline $\mathrm{C}$ & -5.0150820000 & 5.1021910000 & 0.2161750000 \\
\hline $\mathrm{C}$ & -5.5404810000 & 2.7973100000 & 0.7750530000 \\
\hline $\mathrm{C}$ & -5.1603460000 & -0.9620750000 & 1.1664670000 \\
\hline $\mathrm{C}$ & -5.2216300000 & -1.3876900000 & -0.1603850000 \\
\hline $\mathrm{C}$ & -5.3106590000 & -0.4488890000 & -1.2035870000 \\
\hline $\mathrm{C}$ & -5.4068840000 & 0.9096990000 & -0.8714020000 \\
\hline $\mathrm{C}$ & -5.3611060000 & 1.3388040000 & 0.4570980000 \\
\hline $\mathrm{C}$ & -5.2080740000 & 0.3972510000 & 1.4921760000 \\
\hline $\mathrm{C}$ & -5.0829280000 & 0.8175320000 & 5690000 \\
\hline $\mathrm{C}$ & -2.0405310000 & 0.8402530000 & 2.8669090000 \\
\hline $\mathrm{C}$ & -1.8912650000 & -1.2068820000 & 430000 \\
\hline $\mathrm{C}$ & -2.0406680000 & -0.2224830000 & -1.3252580000 \\
\hline $\mathrm{C}$ & -2.0042220000 & 1.1241840000 & -0.9171400000 \\
\hline $\mathrm{C}$ & -2.0141700000 & 1.4136870000 & 670000 \\
\hline $\mathrm{C}$ & -1.9318350000 & 0.4281500000 & 20000 \\
\hline $\mathrm{C}$ & -1.7860670000 & -0.9099680000 & 520000 \\
\hline $\mathrm{C}$ & -1.8555650000 & -4.2936600000 & 2.6965060000 \\
\hline $\mathrm{C}$ & -1.4690150000 & -2.0172240000 & 1.9742240000 \\
\hline $\mathrm{C}$ & 1.3507730000 & 0.1175290000 & 5.1766550000 \\
\hline $\mathrm{C}$ & 6.3418650000 & -4.5504210000 & -1.9437060000 \\
\hline $\mathrm{C}$ & 5.3088550000 & -2.3608960000 & -1.6510180000 \\
\hline $\mathrm{C}$ & 5.3413300000 & 4.1656680000 & 3.0137670000 \\
\hline $\mathrm{C}$ & 5.6545210000 & 2.6983180000 & 1210000 \\
\hline $\mathrm{C}$ & 5.1864130000 & -2.2512900000 & 1.4854870000 \\
\hline $\mathrm{C}$ & 2.1728600000 & -2.3324810000 & 1.7802110000 \\
\hline $\mathrm{C}$ & 2.0723160000 & -1.7253190000 & -4.7628500000 \\
\hline $\mathrm{C}$ & 1.5091040000 & -0.4373530000 & -2.7797720000 \\
\hline $\mathrm{C}$ & 2.1568940000 & 0.4757330000 & 2.9038150000 \\
\hline $\mathrm{C}$ & 1.7203850000 & -0.1903090000 & -1.3205310000 \\
\hline $\mathrm{C}$ & 1.7904740000 & -1.2880570000 & -0.4601770000 \\
\hline $\mathrm{C}$ & 1.9694040000 & -1.1251140000 & 0.9193350000 \\
\hline $\mathrm{C}$ & 1.9946090000 & 0.1797120000 & 1.4414840000 \\
\hline $\mathrm{C}$ & 1.9216550000 & 1.2775460000 & 0.5823400000 \\
\hline $\mathrm{C}$ & 1.8210190000 & 1.1159720000 & -0.8036260000 \\
\hline $\mathrm{C}$ & 1.8625700000 & 2.3215580000 & -1.6976480000 \\
\hline $\mathrm{C}$ & 4.8941740000 & 2.5544880000 & -1.7676850000 \\
\hline $\mathrm{C}$ & 5.2024500000 & -1.1152130000 & -0.8292480000 \\
\hline $\mathrm{C}$ & 5.2456230000 & -1.0560720000 & 0.5790050000 \\
\hline
\end{tabular}




\begin{tabular}{|c|c|c|c|}
\hline $\mathrm{C}$ & 5.3276530000 & 0.2071500000 & 1.1808210000 \\
\hline $\mathrm{C}$ & 5.3173430000 & 1.3931240000 & 0.4460620000 \\
\hline 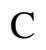 & 5.0918550000 & 1.3271340000 & -0.9416100000 \\
\hline 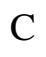 & 5.0552750000 & 0.0731470000 & -1.5498290000 \\
\hline $\mathrm{H}$ & 7.1333280000 & -5.1328400000 & -1.4698710000 \\
\hline & 6.5485610000 & -4.4594130000 & -3.0132210000 \\
\hline $\mathrm{H}$ & 5.3809220000 & -5.0539140000 & -1.8095200000 \\
\hline $\mathrm{H}$ & 6.9508100000 & -2.9919410000 & -0.6088880000 \\
\hline $\mathrm{H}$ & 4.8718680000 & 4.1173050000 & 3.9972490000 \\
\hline $\mathrm{H}$ & 4.8940340000 & 4.9780280000 & 2.4310600000 \\
\hline$H$ & 6.4084940000 & 4.3710940000 & 3.1279930000 \\
\hline $\mathrm{H}$ & 4.3583010000 & 2.3106770000 & 2.6504350000 \\
\hline 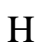 & 1.1940640000 & 1.1773130000 & 5.3995660000 \\
\hline $\mathrm{H}$ & 2.3251490000 & -0.1652400000 & 5.5859320000 \\
\hline $\mathrm{H}$ & 0.5705060000 & -0.4768810000 & 5.6536350000 \\
\hline $\mathrm{H}$ & 2.8557200000 & -2.4315570000 & -5.0362440000 \\
\hline $\mathrm{H}$ & 2.1386710000 & 5630000 & 580000 \\
\hline $\mathrm{H}$ & 1.0926820000 & -2.1831280000 & -4.9304570000 \\
\hline $\mathrm{H}$ & 0.5616180000 & -0.7467270000 & 3.3738830000 \\
\hline $\mathrm{H}$ & 3.0485510000 & -1.8262950000 & -2.8822980000 \\
\hline $\mathrm{H}$ & 4.8398130000 & 2.3390550000 & -2.8371550000 \\
\hline $\mathrm{H}$ & 5.6646100000 & 720000 & 30000 \\
\hline $\mathrm{H}$ & 5.1705150000 & -1.9468510000 & 2.5351490000 \\
\hline $\mathrm{H}$ & 6.0021560000 & -2.9621140000 & 1.3473060000 \\
\hline $\mathrm{H}$ & 0.9968060000 & 2.9682300000 & -1.5384630000 \\
\hline $\mathrm{H}$ & 1.9064560000 & 2.0726390000 & -2.7575290000 \\
\hline $\mathrm{H}$ & 2.3108680000 & 320000 & 50000 \\
\hline $\mathrm{H}$ & 1.3518700000 & -3.0455200000 & 1.6940780000 \\
\hline $\mathrm{H}$ & 5.4089610000 & 0.2745050000 & 2.2606160000 \\
\hline $\mathrm{H}$ & 4.9285900000 & -0.0002910000 & -2.6255750000 \\
\hline $\mathrm{H}$ & 1.7682160000 & -2.2923100000 & -0.8668690000 \\
\hline $\mathrm{H}$ & 2.0304170000 & 2.2793810000 & 0.9842560000 \\
\hline $\mathrm{H}$ & -2.1670760000 & 3.3648510000 & -4.3321570000 \\
\hline $\mathrm{H}$ & 0000 & 3.0598230000 & 430000 \\
\hline $\mathrm{H}$ & -0.9288980000 & 4.2830960000 & -3.4500380000 \\
\hline $\mathrm{H}$ & -0.5113650000 & 1.4746530000 & -3.0061950000 \\
\hline $\mathrm{H}$ & -2.4018870000 & -5.1633890000 & 2.3308740000 \\
\hline $\mathrm{H}$ & -2.2298530000 & -4.0064240000 & 3.6839370000 \\
\hline $\mathrm{H}$ & -0.7950930000 & -4.5424640000 & 2.7871760000 \\
\hline $\mathrm{H}$ & -2.8367920000 & -3.2653040000 & 1.1097630000 \\
\hline $\mathrm{H}$ & -7.0917180000 & -5.0162240000 & -2.0531910000 \\
\hline $\mathrm{H}$ & -6.5662650000 & -5.3903710000 & -0.3908220000 \\
\hline $\mathrm{H}$ & -5.3611180000 & -5.2017790000 & -1.6718350000 \\
\hline $\mathrm{H}$ & -6.0387370000 & 5.4229230000 & 0.0009160000 \\
\hline $\mathrm{H}$ & -4.3226790000 & 5.6139900000 & -0.4522890000 \\
\hline $\mathrm{H}$ & -4.7944060000 & 5.3726940000 & 1.2538400000 \\
\hline $\mathrm{H}$ & -7.0301910000 & -2.8207440000 & -1.2326960000 \\
\hline
\end{tabular}




$\begin{array}{lrrc}\mathrm{H} & -4.1379520000 & 3.3759770000 & -0.6538000000 \\ \mathrm{H} & -2.1216440000 & 1.9242610000 & 2.9739790000 \\ \mathrm{H} & -1.2146440000 & 0.4726060000 & 3.4737600000 \\ \mathrm{H} & -2.2997850000 & -1.7779140000 & -2.7838760000 \\ \mathrm{H} & -1.4430420000 & -0.3601470000 & -3.4130050000 \\ \mathrm{H} & -5.8844040000 & 0.3982490000 & 3.5389290000 \\ \mathrm{H} & -5.0856360000 & 1.8980860000 & 3.0596140000 \\ \mathrm{H} & -5.1785370000 & -1.9412910000 & -2.7864870000 \\ \mathrm{H} & -6.1110950000 & -0.4862680000 & -3.2106550000 \\ \mathrm{H} & -1.8535650000 & -2.2468740000 & -0.6468020000 \\ \mathrm{H} & -2.0961480000 & 2.4569350000 & 0.7376310000 \\ \mathrm{H} & -5.4666040000 & 1.6401970000 & -1.6702430000 \\ \mathrm{H} & -5.0139500000 & -1.6846630000 & 1.9621630000\end{array}$

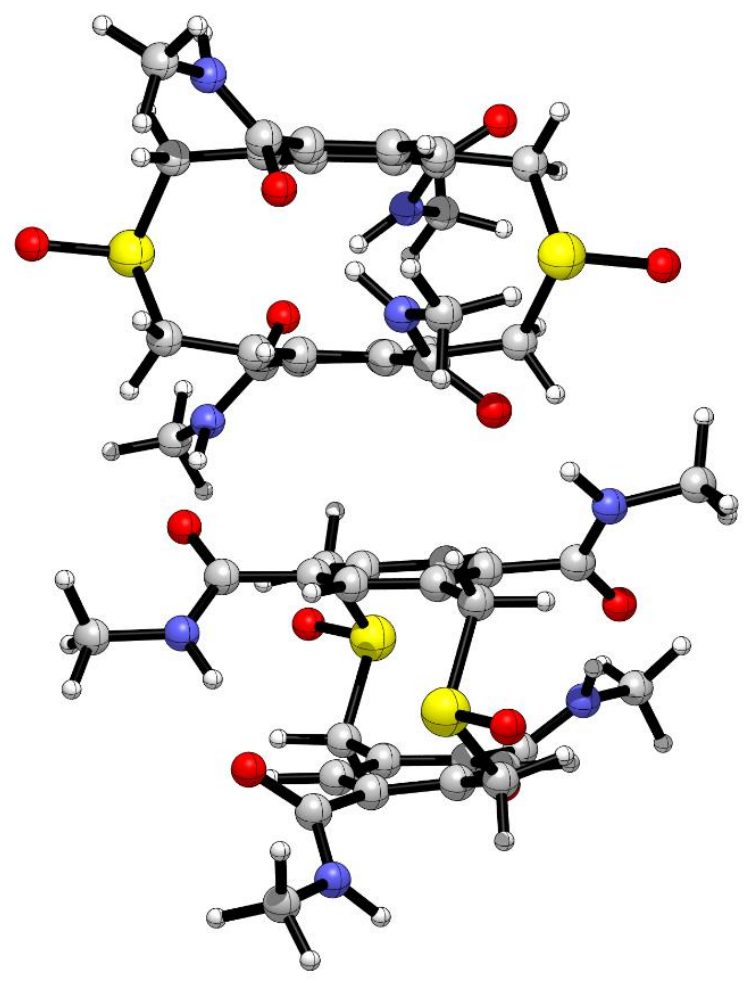

sulfoxide chair anti-dimer

$S=O$ away

Energy: -4794.13508166

$\begin{array}{lrrr}\text { S } & 4.1915190000 & -3.3964290000 & -0.4200730000 \\ \text { S } & 2.7018350000 & 3.7847230000 & 0.2018170000 \\ \text { S } & -4.2508980000 & 1.6001900000 & -3.0333970000 \\ \text { S } & -2.6966250000 & -1.8323700000 & 3.2751910000 \\ \text { O } & 4.6079520000 & -4.9039120000 & 0.1643830000 \\ \text { O } & 2.2352160000 & 5.2073720000 & -0.5494430000 \\ \text { O } & -4.6747580000 & 1.5000080000 & -4.6452970000 \\ \text { O } & -2.2458980000 & -1.9401460000 & 4.8839140000 \\ \text { O } & -5.3922580000 & 1.8318980000 & 3.6319120000 \\ \text { O } & -2.9100490000 & 3.5531950000 & -0.3037310000\end{array}$




\begin{tabular}{|c|c|c|c|}
\hline 9 & -4.4195810000 & -3.2690250000 & -1 \\
\hline C & -0.3703890000 & -2.9951120000 & 13874800000 \\
\hline & 0.3699080000 & 1.3570780000 & 760000 \\
\hline & 4.4866020000 & -1.2979220000 & -3.2980530000 \\
\hline & 5.5628460000 & 3.6073770000 & 1.8966050000 \\
\hline & 2.9932070000 & -0.3113360000 & 70000 \\
\hline & -6.4772580000 & -2.5333500000 & -2.0301230000 \\
\hline & -1.3846960000 & 3.2355390000 & 800000 \\
\hline & -4.3210500000 & 3.0858090000 & 560000 \\
\hline & 32430000 & -3.657 & 000 \\
\hline & 70000 & 300000 & 0000 \\
\hline & 00000 & -2.021 & 890000 \\
\hline & 4.4255630000 & 2.08 & 720000 \\
\hline & 820000 & 0.04 & 0000 \\
\hline & -6.6 & -3.6 & 0000 \\
\hline & 140000 & 0000 & 60000 \\
\hline & -2.1 & 2.80 & 10000 \\
\hline $\mathrm{C}$ & 8070000 & 0.50 & 00000 \\
\hline & $-5.5^{\circ}$ & 0.44 & -2.2 \\
\hline $\mathrm{C}$ & -5.3 & -2.4 & 000 \\
\hline $\mathrm{C}$ & -4.2 & 4.25 & 0000 \\
\hline $\mathrm{C}$ & -4.9 & 1.95 & 0000 \\
\hline $\mathrm{C}$ & -4.93 & -1.51 & 000 \\
\hline $\mathrm{C}$ & -5.23 & -1.24 & -0.3 \\
\hline $\mathrm{C}$ & -5.3 & & -0.8 \\
\hline $\mathrm{C}$ & $-5.2^{\top}$ & 1.05 & 0000 \\
\hline $\mathrm{C}$ & -4.9 & 0.8 & 000 \\
\hline $\mathrm{C}$ & -4.78 & 50000 & 000 \\
\hline $\mathrm{C}$ & -4.35 & -0.87 & 0000 \\
\hline $\mathrm{C}$ & -1.5 & $-0.4^{\prime}$ & 000 \\
\hline $\mathrm{C}$ & $-1.92^{\prime}$ & -1.00 & 0000 \\
\hline $\mathrm{C}$ & -2.1 & 0.2 & 000 \\
\hline$C$ & -2.02 & 0000 & -0.6 \\
\hline $\mathrm{C}$ & -1.78 & 1.05 & 000 \\
\hline $\mathrm{C}$ & 60000 & 80000 & 0000 \\
\hline $\mathrm{C}$ & -1.6 & -1.29 & 30000 \\
\hline $\mathrm{C}$ & $-1.8^{\prime}$ & -5.0 & 30000 \\
\hline $\mathrm{C}$ & -1.316 & -2.70 & 000 \\
\hline $\mathrm{C}$ & 1.36 & -2.35 & 0000 \\
\hline $\mathrm{C}$ & 6.650 & -2.99 & 2060000 \\
\hline $\mathrm{C}$ & 5.34 & -1.25 & 350000 \\
\hline $\mathrm{C}$ & 90000 & 90000 & 10000 \\
\hline $\mathrm{C}$ & 5.02 & 2.48 & 70000 \\
\hline $\mathrm{C}$ & 450000 & -2.2619490000 & 0.5006350000 \\
\hline $\mathrm{C}$ & 2.5734800000 & -2.986 & 0.5022170000 \\
\hline $\mathrm{C}$ & 1.8888770000 & 0.3160540000 & -5.1121030000 \\
\hline $\mathrm{C}$ & 1.3153350000 & 0.5747330000 & -2.7626760000 \\
\hline
\end{tabular}




\begin{tabular}{|c|c|c|c|}
\hline $\mathrm{C}$ & 2.2087900000 & -0.9775730000 & 2.7530700000 \\
\hline $\mathrm{C}$ & 1.5972150000 & 0.1912530000 & -1.3415770000 \\
\hline 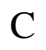 & 1.8725570000 & -1.1447100000 & -1.0406120000 \\
\hline $\mathrm{C}$ & 2.1302660000 & -1.5747060000 & 0.2650090000 \\
\hline $\mathrm{C}$ & 2.0321020000 & -0.6326270000 & 1.3013650000 \\
\hline $\mathrm{C}$ & 1.7816860000 & 0.7062420000 & 0.9991900000 \\
\hline $\mathrm{C}$ & 1.5902420000 & 1.1491990000 & -0.3108420000 \\
\hline $\mathrm{C}$ & 1.4316940000 & 2.6114720000 & -0.5909330000 \\
\hline $\mathrm{C}$ & 4.3021050000 & 3.3068040000 & -0.7314170000 \\
\hline $\mathrm{C}$ & 5.2182030000 & -0.3518540000 & -1.2004260000 \\
\hline $\mathrm{C}$ & 5.3365980000 & -0.8077660000 & 0.1253620000 \\
\hline $\mathrm{C}$ & 5.2727290000 & 0.1425250000 & 1.1494300000 \\
\hline $\mathrm{C}$ & 5.0280000000 & 1.4918020000 & 0.8974960000 \\
\hline $\mathrm{C}$ & 4.7688350000 & 1.9171630000 & -0.4195420000 \\
\hline $\mathrm{C}$ & 4.8895400000 & 0.9832030000 & -1.4483440000 \\
\hline $\mathrm{H}$ & 7.5898920000 & -3.5223210000 & -3.3432040000 \\
\hline $\mathrm{H}$ & 6.6792420000 & -2.5018530000 & -4.4875170000 \\
\hline $\mathrm{H}$ & 5.8293480000 & -3.7167590000 & -3.5146400000 \\
\hline $\mathrm{H}$ & 7.1884600000 & -1.8849600000 & -1.7656900000 \\
\hline $\mathrm{H}$ & 3.8351640000 & 2.4622990000 & 5.1207580000 \\
\hline $\mathrm{H}$ & 3.8892090000 & 3.9084980000 & 4.0745030000 \\
\hline $\mathrm{H}$ & 5.3948540000 & 80000 & 160000 \\
\hline $\mathrm{H}$ & 3.9357580000 & 1.1859130000 & 3.2246260000 \\
\hline $\mathrm{H}$ & 0.3174410000 & -2.3616870000 & 4.9391170000 \\
\hline $\mathrm{H}$ & 1.9214070000 & -1.6189140000 & 5.2112160000 \\
\hline $\mathrm{H}$ & 1.8042440000 & -3.3403080000 & 4.7791270000 \\
\hline $\mathrm{H}$ & 2.6738560000 & 10000 & -5.6812810000 \\
\hline $\mathrm{H}$ & 1.9156950000 & 1.3913600000 & -5.3104580000 \\
\hline $\mathrm{H}$ & 0.9124610000 & -0.0588580000 & -5.4315610000 \\
\hline $\mathrm{H}$ & 0.7892670000 & -2.4264930000 & 2.5617250000 \\
\hline $\mathrm{H}$ & 2.9457340000 & -0.4958330000 & -3.4421470000 \\
\hline $\mathrm{H}$ & 4.0709320000 & 3.4576110000 & -1.7879270000 \\
\hline $\mathrm{H}$ & 4.9971110000 & 4.0675180000 & -0.3753090000 \\
\hline $\mathrm{H}$ & 5.2351100000 & -2.4182140000 & 1.5648790000 \\
\hline $\mathrm{H}$ & 6.3724080000 & -2.7485900000 & 0.2548690000 \\
\hline $\mathrm{H}$ & 0.5028950000 & 3.0155540000 & -0.1834420000 \\
\hline $\mathrm{H}$ & 1.4673840000 & 2.8670140000 & -1.6485800000 \\
\hline $\mathrm{H}$ & 2.7713360000 & -3.2298270000 & 1.5455150000 \\
\hline $\mathrm{H}$ & 1.8706670000 & -3.7223310000 & 0.1089950000 \\
\hline $\mathrm{H}$ & 5.3802560000 & -0.1866130000 & 2.1773060000 \\
\hline $\mathrm{H}$ & 4.7065940000 & 1.2892600000 & -2.4737360000 \\
\hline $\mathrm{H}$ & 1.9101000000 & -1.8677120000 & -1.8495610000 \\
\hline $\mathrm{H}$ & 1.7699440000 & 1.4246410000 & 1.8132190000 \\
\hline $\mathrm{H}$ & -2.1113680000 & 5.1871880000 & -1.9153850000 \\
\hline $\mathrm{H}$ & -1.2639980000 & 4.7555240000 & -3.4226920000 \\
\hline $\mathrm{H}$ & -0.3339210000 & 5.0584700000 & -1.9275050000 \\
\hline $\mathrm{H}$ & -0.7350530000 & 2.5707540000 & -2.4162200000 \\
\hline
\end{tabular}




$\begin{array}{lrrr}\mathrm{H} & -2.6273760000 & -5.6523990000 & -0.1258490000 \\ \mathrm{H} & -1.9525050000 & -5.2251520000 & 1.4715330000 \\ \mathrm{H} & -0.8770030000 & -5.3819140000 & 0.0767760000 \\ \mathrm{H} & -2.9128070000 & -3.4176580000 & -0.4954950000 \\ \mathrm{H} & -7.4381850000 & -3.3213850000 & -3.7072930000 \\ \mathrm{H} & -7.0304940000 & -4.5312010000 & -2.4620190000 \\ \mathrm{H} & -5.7526890000 & -3.8491170000 & -3.4815330000 \\ \mathrm{H} & -5.2464020000 & 4.6359130000 & 3.1443840000 \\ \mathrm{H} & -3.6676930000 & 5.0205260000 & 2.4087810000 \\ \mathrm{H} & -3.7676180000 & 3.9976290000 & 3.8694900000 \\ \mathrm{H} & -7.2279000000 & -1.8828210000 & -1.8599790000 \\ \mathrm{H} & -3.8709800000 & 3.1551710000 & 1.1318820000 \\ \mathrm{H} & -1.7753030000 & 0.4257960000 & 3.2031350000 \\ \mathrm{H} & -0.5592690000 & -0.8459850000 & 2.9706160000 \\ \mathrm{H} & -2.9639030000 & -0.4270120000 & -3.4486830000 \\ \mathrm{H} & -1.9886930000 & 1.0495360000 & -3.5976420000 \\ \mathrm{H} & -5.0313760000 & -1.5837900000 & 3.7685090000 \\ \mathrm{H} & -4.2261710000 & -0.0229560000 & 3.9569710000 \\ \mathrm{H} & -5.5845770000 & -0.4031120000 & -2.9439130000 \\ \mathrm{H} & -6.4769270000 & 1.0378140000 & -2.4304940000 \\ \mathrm{H} & -2.0076650000 & -1.8199830000 & -1.8309900000 \\ \mathrm{H} & -1.7766560000 & 1.8719660000 & 1.4108000000 \\ \mathrm{H} & -5.4012800000 & 2.1293610000 & -0.1588690000 \\ \mathrm{H} & -4.7783820000 & -2.5544420000 & 1.2392920000\end{array}$




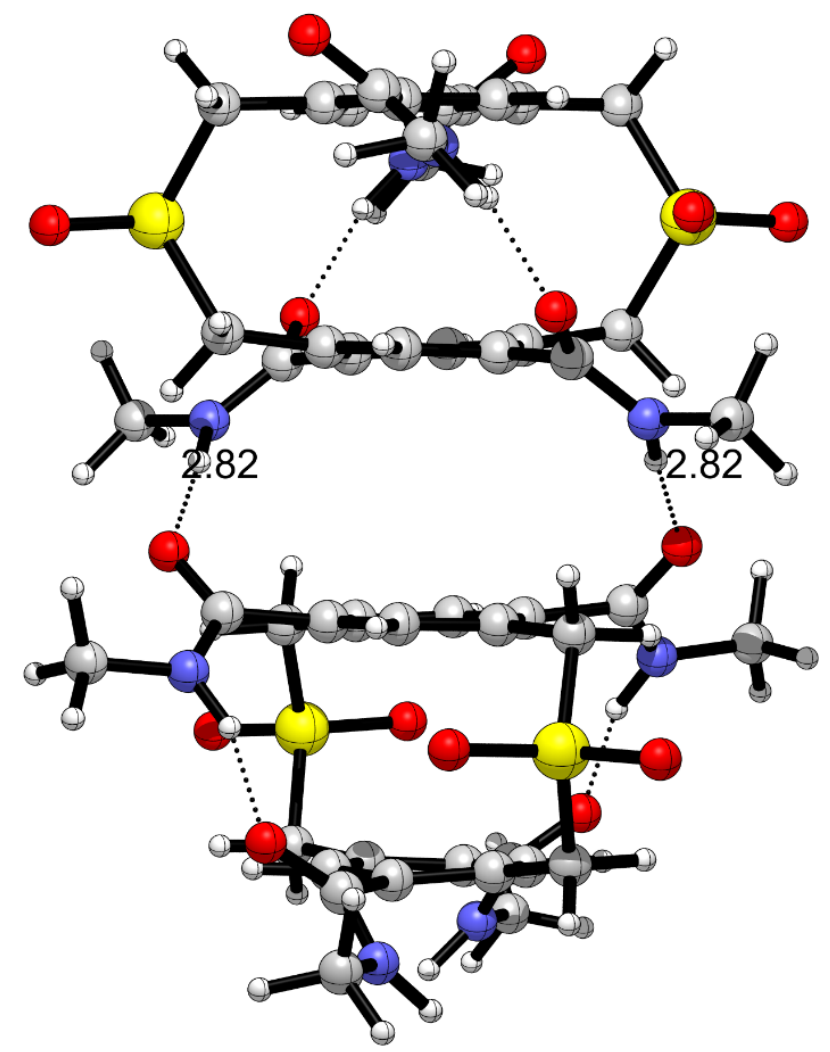

$\left(S_{\mathrm{p}}\right)$-dt[3.3]pCpTA-SO $\mathrm{O}_{2}$ 6-3 chair syn- dimer

Energy: -5094.48113661

$\begin{array}{lrrr}\mathrm{S} & -3.7743840000 & 1.5546170000 & -3.3503640000 \\ \mathrm{~S} & -3.7724130000 & -1.5554770000 & 3.3505240000 \\ \mathrm{~S} & 3.4247380000 & 3.6809190000 & 0.0535570000 \\ \mathrm{~S} & 3.4268910000 & -3.6804410000 & -0.0534890000 \\ \mathrm{O} & -3.7084950000 & 1.5669060000 & -4.9786440000 \\ \mathrm{O} & -3.8060740000 & 2.9766240000 & -2.5430960000 \\ \mathrm{O} & -3.7058950000 & -1.5680940000 & 4.9787790000 \\ \mathrm{O} & -3.8039250000 & -2.9773170000 & 2.5429650000 \\ \mathrm{O} & 3.4906750000 & 5.1771180000 & -0.5827010000 \\ \mathrm{O} & 3.3607070000 & 3.4889970000 & 1.6747720000 \\ \mathrm{O} & 3.4937670000 & -5.1764840000 & 0.5829960000 \\ \mathrm{O} & 3.3618870000 & -3.4887640000 & -1.6747030000 \\ \mathrm{O} & -6.1729280000 & 2.1224790000 & 2.9452660000 \\ \mathrm{O} & -2.4507220000 & -3.6392610000 & -0.0527330000 \\ \mathrm{O} & -6.1730790000 & -2.1239950000 & -2.9439240000 \\ \mathrm{O} & -2.4523630000 & 3.6391120000 & 0.0527520000 \\ \mathrm{O} & 0.7862330000 & 1.6380550000 & -3.1478490000 \\ \mathrm{O} & 4.6422100000 & 1.4224050000 & 3.3369160000 \\ \mathrm{O} & 4.6411760000 & -1.4216990000 & -3.3378350000 \\ \mathrm{O} & 0.7875450000 & -1.6380780000 & 3.1487280000 \\ \mathrm{~N} & -4.6789690000 & -3.2616630000 & -1.6494890000 \\ \mathrm{~N} & -0.9968170000 & -3.2246980000 & 1.6481280000\end{array}$




\begin{tabular}{|c|c|c|c|}
\hline $\mathrm{N}$ & -4.6798350000 & 3.2607990000 & 1.6502280000 \\
\hline & -0.9988860000 & 3.2253170000 & -1.6486250000 \\
\hline $\mathrm{N}$ & 2.3778870000 & -0.1035360000 & 3.6818550000 \\
\hline N & 6.2210120000 & -2.7348870000 & -2.3689410000 \\
\hline $\mathrm{N}$ & 6.2204790000 & 2.7369030000 & 2.3671870000 \\
\hline $\mathrm{N}$ & 2.3756890000 & 0.1028580000 & -3.6816200000 \\
\hline $\mathrm{C}$ & -4.7211080000 & -4.4337420000 & -2.5108030000 \\
\hline $\mathrm{C}$ & -0.9965430000 & -4.6106490000 & 2.0985950000 \\
\hline C & -1.8326750000 & -2.8233830000 & 0.6790170000 \\
\hline $\mathrm{C}$ & -2.2148880000 & -0.5703620000 & 2.8486850000 \\
\hline $\mathrm{C}$ & -5.3476730000 & -0.6311220000 & 2.8376060000 \\
\hline $\mathrm{C}$ & -4.7221300000 & 4.4328640000 & 2.5115510000 \\
\hline $\mathrm{C}$ & -5.4426090000 & 2.1859540000 & 1.9228720000 \\
\hline $\mathrm{C}$ & -5.4422750000 & -2.1871280000 & -1.9218550000 \\
\hline $\mathrm{C}$ & -5.3824210000 & 450000 & -0.4286910000 \\
\hline $\mathrm{C}$ & -5.3786910000 & 1.0480460000 & 0.9417880000 \\
\hline $\mathrm{C}$ & -5.3746250000 & -0.2891870000 & 1.3791190000 \\
\hline $\mathrm{C}$ & -5.3815710000 & -1.3170220000 & 0.4297180000 \\
\hline $\mathrm{C}$ & -5.3785230000 & -1.0492170000 & -0.9407640000 \\
\hline $\mathrm{C}$ & -5.3753230000 & 20000 & 00000 \\
\hline $\mathrm{C}$ & -5.3491450000 & 0.6298870000 & -2.8366040000 \\
\hline $\mathrm{C}$ & -2.2162220000 & 0.5702990000 & -2.8489510000 \\
\hline $\mathrm{C}$ & -1.9810700000 & 0.9912480000 & 0.9420710000 \\
\hline $\mathrm{C}$ & -2.0174410000 & 880000 & 310000 \\
\hline $\mathrm{C}$ & -1.9196670000 & -1.347 & 620000 \\
\hline $\mathrm{C}$ & -1.9809730000 & -0.9911760000 & -0.9424080000 \\
\hline $\mathrm{C}$ & -2.0181640000 & 10000 & 3070000 \\
\hline $\mathrm{C}$ & -1.9204870000 & 1.3480900000 & -0.4064100000 \\
\hline $\mathrm{C}$ & -1.8342530000 & 2.8235510000 & -0.6792790000 \\
\hline $\mathrm{C}$ & -0.9989810000 & 4.6114340000 & -2.0985300000 \\
\hline $\mathrm{C}$ & 2.2250650000 & -0.3472450000 & 5.1123870000 \\
\hline $\mathrm{C}$ & 6.2175100000 & -3.6930690000 & -3.4705920000 \\
\hline $\mathrm{C}$ & 5.3351990000 & -1.7226050000 & -2.3374220000 \\
\hline $\mathrm{C}$ & 6.2168780000 & 50000 & 520000 \\
\hline $\mathrm{C}$ & 5.3353590000 & 1.7240060000 & 2.3360950000 \\
\hline $\mathrm{C}$ & 5.0281950000 & 2.8510090000 & -0.5727100000 \\
\hline $\mathrm{C}$ & 1.8963730000 & 2.8267030000 & -0.6792130000 \\
\hline $\mathrm{C}$ & 2.2221610000 & 0.3464800000 & -5.1120840000 \\
\hline $\mathrm{C}$ & 1.6202140000 & 0.7504810000 & -2.7934670000 \\
\hline $\mathrm{C}$ & 1.6216950000 & -0.7507730000 & 2.7940550000 \\
\hline $\mathrm{C}$ & 1.7748540000 & -0.9673870000 & -0.9925180000 \\
\hline $\mathrm{C}$ & 1.7664100000 & 0.3824360000 & -1.3485090000 \\
\hline $\mathrm{C}$ & 1.8065670000 & 1.3685080000 & -0.3460170000 \\
\hline $\mathrm{C}$ & 1.7745240000 & 0.9671780000 & 0.9930710000 \\
\hline $\mathrm{C}$ & 1.7672150000 & -0.3826460000 & 1.3490470000 \\
\hline $\mathrm{C}$ & 1.8077100000 & -1.3686920000 & 0.3465590000 \\
\hline $\mathrm{C}$ & 1.8984610000 & -2.8268120000 & 0.6798270000 \\
\hline
\end{tabular}




\begin{tabular}{|c|c|c|c|}
\hline $\mathrm{C}$ & 5.0304420000 & -2.8497370000 & 0.5714670000 \\
\hline & 5.2059170000 & 0.4863070000 & -1.2826030000 \\
\hline C & 5.1883010000 & 1.4029560000 & -0.2270820000 \\
\hline & 5.2670860000 & 0.8953320000 & 1.0852970000 \\
\hline $\mathrm{C}$ & 5.2069590000 & -0.4849210000 & 1.2812980000 \\
\hline $\mathrm{C}$ & 5.1893770000 & -1.4015750000 & 0.2257880000 \\
\hline $\mathrm{C}$ & 5.2670990000 & -0.8939030000 & -1.0866280000 \\
\hline $\mathrm{H}$ & 1.2110040000 & -0.1025680000 & 5.4388300000 \\
\hline H & 2.9418120000 & 0.2807520000 & 5.6401240000 \\
\hline $\mathrm{H}$ & 2.4082440000 & -1.3989170000 & 5.3494340000 \\
\hline $\mathrm{H}$ & 3.1257960000 & 0.5388920000 & 3.3967100000 \\
\hline $\mathrm{H}$ & 1.2080430000 & 0.1014450000 & -5.4380880000 \\
\hline $\mathrm{H}$ & 2.9388790000 & -0.2812920000 & -5.6401260000 \\
\hline $\mathrm{H}$ & 2.4048750000 & 1.3982090000 & -5.3492330000 \\
\hline $\mathrm{H}$ & 3.1239760000 & -0.5392530000 & -3.3967810000 \\
\hline $\mathrm{H}$ & 5.2301720000 & 4.1603980000 & 3.5301300000 \\
\hline $\mathrm{H}$ & 6.9707480000 & 4.4578850000 & 3.2744180000 \\
\hline $\mathrm{H}$ & 6.4365630000 & 3.1972860000 & 4.4160370000 \\
\hline $\mathrm{H}$ & 5.2302960000 & -4.1571250000 & -3.5329280000 \\
\hline $\mathrm{H}$ & 080000 & 60000 & -3.2751760000 \\
\hline $\mathrm{H}$ & 6.4389480000 & -3.1956950000 & -4.4176100000 \\
\hline $\mathrm{H}$ & 6.8877250000 & 2.8465690000 & 1.6222430000 \\
\hline $\mathrm{H}$ & 6.8890170000 & -2.8438080000 & -1.6245640000 \\
\hline $\mathrm{H}$ & 1.9472740000 & -3.0473680000 & 1.7444840000 \\
\hline $\mathrm{H}$ & 1.0982410000 & -3.4230 & 0.2356560000 \\
\hline $\mathrm{H}$ & 1.0960330000 & 3.4225420000 & -0.2347200000 \\
\hline $\mathrm{H}$ & 1.9447290000 & 3.0473380000 & -1.7438710000 \\
\hline $\mathrm{H}$ & 4.9934120000 & -3.0200340000 & 1.6483240000 \\
\hline $\mathrm{H}$ & 5.7654700000 & -3.5272950000 & 0.1360790000 \\
\hline $\mathrm{H}$ & 5.7632630000 & 3.5290500000 & -0.1381420000 \\
\hline $\mathrm{H}$ & 4.9900380000 & 3.0211850000 & -1.6495500000 \\
\hline $\mathrm{H}$ & 1.7805470000 & 1.7250420000 & 1.7689980000 \\
\hline $\mathrm{H}$ & 1.7810570000 & -1.7252820000 & -1.7684160000 \\
\hline $\mathrm{H}$ & 5.1569740000 & 0.8455880000 & -2.3056300000 \\
\hline $\mathrm{H}$ & 5.1588540000 & -0.8442370000 & 2.3043520000 \\
\hline $\mathrm{H}$ & -5.7088360000 & -4.9030370000 & -2.4824240000 \\
\hline $\mathrm{H}$ & -3.9709320000 & -5.1403810000 & -2.1553950000 \\
\hline $\mathrm{H}$ & -4.5123990000 & -4.1631170000 & -3.5504800000 \\
\hline $\mathrm{H}$ & -3.9862770000 & -3.2742280000 & -0.8966930000 \\
\hline $\mathrm{H}$ & -5.7099360000 & 4.9020050000 & 2.4832510000 \\
\hline $\mathrm{H}$ & -3.9721040000 & 5.1396450000 & 2.1561010000 \\
\hline $\mathrm{H}$ & -4.5133040000 & 4.1622770000 & 3.5512130000 \\
\hline $\mathrm{H}$ & -3.9873440000 & 3.2736020000 & 0.8972360000 \\
\hline $\mathrm{H}$ & -1.9845700000 & -4.8628050000 & 2.4929520000 \\
\hline $\mathrm{H}$ & -0.2487230000 & -4.7151540000 & 2.8858400000 \\
\hline $\mathrm{H}$ & -0.7593960000 & -5.2898240000 & 1.2754030000 \\
\hline $\mathrm{H}$ & -1.9870170000 & 4.8635580000 & -2.4928790000 \\
\hline
\end{tabular}




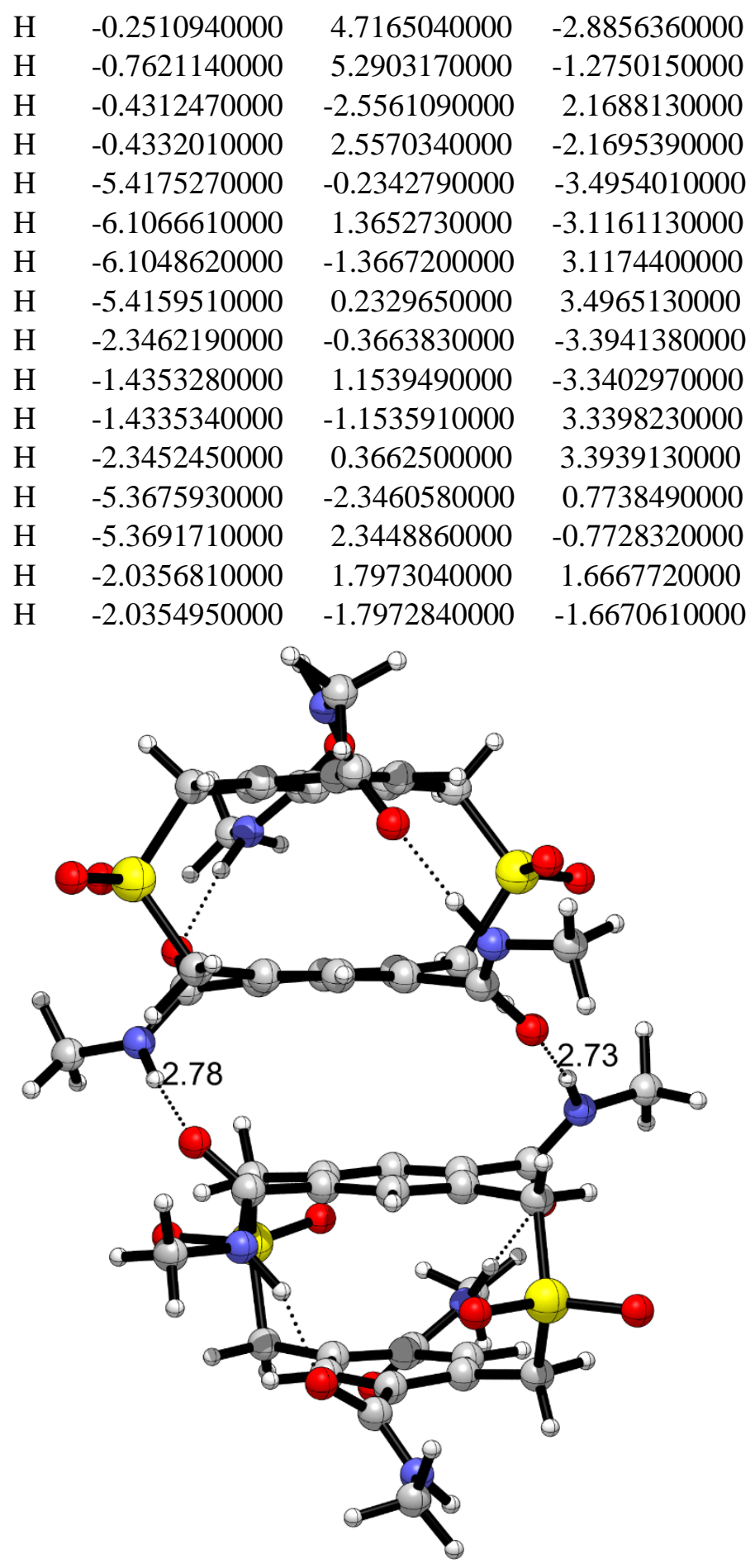

$\left(S_{\mathrm{p}}\right)$-dtt3.3]pCpTA-SO $\mathrm{O}_{2}$ 6-3 chair anti- dimer

Energy: -5094.48113661

$\begin{array}{lrrr}\mathrm{S} & 4.2020660000 & -3.4621070000 & -0.1441220000 \\ \mathrm{~S} & 2.7144420000 & 3.7649480000 & -0.0096680000 \\ \mathrm{~S} & -4.3481710000 & 1.0678140000 & -3.2707300000 \\ \mathrm{~S} & -2.8694810000 & -1.3252360000 & 3.5554060000 \\ \mathrm{O} & 4.6289030000 & -4.8938610000 & 0.5061140000\end{array}$




\begin{tabular}{|c|c|c|c|}
\hline J & 4.0098550000 & 3090000 & \\
\hline C & 2.3378800000 & 5.1972980000 & -0.6901560000 \\
\hline & 2.6552730000 & 3.5896640000 & 1070000 \\
\hline & -4.6558850000 & 0.8154760000 & -4.8511240000 \\
\hline $\mathrm{O}$ & -4.4152730000 & 2.5786440000 & -2.6575200000 \\
\hline & -2.4532910000 & -1.2485050000 & 0090000 \\
\hline C & -3.0283550000 & -2.7919790000 & 8560000 \\
\hline & -5.6687540000 & 2.1851980000 & 070000 \\
\hline & -2.7502530000 & 3.5855980000 & 170000 \\
\hline 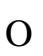 & -4.3957530000 & -3.35810 & 0000 \\
\hline ) & -0.2809570000 & -2.8891220000 & 6770000 \\
\hline ( & 0.34 & 000 & 10000 \\
\hline ) & 4.6839990000 & -1.0371000000 & 980000 \\
\hline & 5.9151220000 & 3.49 & 80000 \\
\hline C & 3.0 & -0.1 & 000 \\
\hline & -6.56 & -2.8 & 170000 \\
\hline & -1.4 & 00 & 50000 \\
\hline & -4.52 & 3.30 & 460000 \\
\hline & -1.9 & -3.68 & 000 \\
\hline & & -1.8 & \\
\hline & $6.5^{\circ}$ & -1.91 & 990000 \\
\hline & 4.7 & & 740000 \\
\hline & 2.15 & -0.0 & 940000 \\
\hline $\mathrm{C}$ & -6.78 & -4.0 & 730000 \\
\hline $\mathrm{C}$ & -1.4 & & -2 \\
\hline $\mathrm{C}$ & -2.12 & 00 & 30000 \\
\hline $\mathrm{C}$ & -2.6 & 00 & 530000 \\
\hline & -5.6 & 00 & 00000 \\
\hline $\mathrm{C}$ & -5.38 & -2.5 & 090000 \\
\hline $\mathrm{C}$ & -4.5 & & 70000 \\
\hline $\mathrm{C}$ & -5.1 & 2.21 & 90000 \\
\hline $\mathrm{C}$ & -5.0 & -1.4 & 10000 \\
\hline & -5.3 & -1.32 & 090000 \\
\hline $\mathrm{C}$ & -5.4 & -0.0 & 960000 \\
\hline$c$ & -5.3 & & 10000 \\
\hline $\mathrm{C}$ & -5.1 & 0.9 & 980000 \\
\hline $\mathrm{C}$ & -4.9 & -0.2 & 550000 \\
\hline $\mathrm{C}$ & -4.54 & -0.45 & 30000 \\
\hline $\mathrm{C}$ & -1.5 & -0.3 & 50000 \\
\hline $\mathrm{C}$ & -1.95 & -1.0707 & 3760000 \\
\hline $\mathrm{C}$ & -2.19 & 0.2 & 430000 \\
\hline $\mathrm{C}$ & -2.0512330000 & 0000 & 4210000 \\
\hline $\mathrm{C}$ & -1.8 & & 0000 \\
\hline $\mathrm{C}$ & 6120000 & -0.2034240000 & 1.1914090000 \\
\hline $\mathrm{C}$ & -1.6445510000 & -1.2954460000 & 0.3033800000 \\
\hline C & -1.7263410000 & -5.0601580000 & 0.6329540000 \\
\hline $\mathrm{C}$ & -1.2718230000 & -2.6845050000 & 0.7332550000 \\
\hline
\end{tabular}




\begin{tabular}{|c|c|c|c|}
\hline C & 1.4400180000 & -2.1575650000 & 4.7255590000 \\
\hline C & 6.7480470000 & -2.8666940000 & -3.6577430000 \\
\hline $\mathrm{C}$ & 5.4760610000 & -1.1390810000 & -2.4937390000 \\
\hline 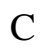 & 4.7879560000 & 2.9594560000 & 4.3785020000 \\
\hline $\mathrm{C}$ & 5.2880170000 & 2.4211450000 & 2.0728370000 \\
\hline $\mathrm{C}$ & 5.5943140000 & -2.2728980000 & 0.4113630000 \\
\hline $\mathrm{C}$ & 2.5532210000 & -2.9612370000 & 0.6474430000 \\
\hline $\mathrm{C}$ & 1.9221500000 & 0.1405670000 & -5.1326460000 \\
\hline C & 1.3343880000 & 0.4461920000 & -2.7932600000 \\
\hline $\mathrm{C}$ & 2.2472400000 & -0.8628060000 & 2.7875160000 \\
\hline $\mathrm{C}$ & 1.6293510000 & 0.1274070000 & -1.3580270000 \\
\hline $\mathrm{C}$ & 1.9075250000 & -1.1941590000 & -1.0029780000 \\
\hline $\mathrm{C}$ & 2.1547490000 & -1.5563900000 & 0.3255040000 \\
\hline $\mathrm{C}$ & 2.0565710000 & -0.5742580000 & 1.3234640000 \\
\hline $\mathrm{C}$ & 1.8004790000 & 0.7503200000 & 80000 \\
\hline $\mathrm{C}$ & 1.6100970000 & 1.1247730000 & -0.3652740000 \\
\hline $\mathrm{C}$ & 1.4256750000 & 2.5684780000 & -0.7207160000 \\
\hline $\mathrm{C}$ & 4.4683690000 & 3.3283420000 & -0.6355790000 \\
\hline $\mathrm{C}$ & 5.2937420000 & -0.3280940000 & -1.2470690000 \\
\hline $\mathrm{C}$ & 5.4214830000 & -0.82 & 910000 \\
\hline $\mathrm{C}$ & 5.3616220000 & 0.0887040000 & 1.1269500000 \\
\hline $\mathrm{C}$ & 5.1422760000 & 1.4513340000 & 0.9281620000 \\
\hline $\mathrm{C}$ & 4.8634940000 & 1.9122900000 & -0.3717060000 \\
\hline $\mathrm{C}$ & 4.9531460000 & 1.0137950000 & -1.4343140000 \\
\hline $\mathrm{H}$ & 7.6794220000 & -3.4122450000 & 5060000 \\
\hline $\mathrm{H}$ & 6.7818430000 & -2.3511550000 & -4.6205050000 \\
\hline $\mathrm{H}$ & 5.9073350000 & -3.5649020000 & -3.6555850000 \\
\hline $\mathrm{H}$ & 7.2908620000 & -1.8328410000 & -1.8639610000 \\
\hline $\mathrm{H}$ & 4.3312140000 & 2.4639880000 & 5.2356720000 \\
\hline $\mathrm{H}$ & 4.2320680000 & 3.8715300000 & 50000 \\
\hline $\mathrm{H}$ & 5.8163330000 & 3.2378870000 & 4.6213420000 \\
\hline $\mathrm{H}$ & 4.1374450000 & 1.2386550000 & 3.2926920000 \\
\hline $\mathrm{H}$ & 0.4228800000 & -2.0444660000 & 5.1131210000 \\
\hline $\mathrm{H}$ & 2.1080660000 & -1.4782820000 & 5.2546320000 \\
\hline $\mathrm{H}$ & 1.7697170000 & -3.1873350000 & 4.8862920000 \\
\hline $\mathrm{H}$ & 2.7599360000 & -0.2937490000 & -5.6770180000 \\
\hline $\mathrm{H}$ & 1.8463110000 & 1.2076500000 & -5.3599710000 \\
\hline $\mathrm{H}$ & 0.9916580000 & -0.3381450000 & -5.4504230000 \\
\hline $\mathrm{H}$ & 0.8042910000 & -2.3055390000 & 2.6793150000 \\
\hline $\mathrm{H}$ & 3.0341150000 & -0.5365040000 & -3.4496870000 \\
\hline $\mathrm{H}$ & 4.4194410000 & 3.5853970000 & -1.6939870000 \\
\hline $\mathrm{H}$ & 5.0785930000 & 4.0383500000 & -0.0716420000 \\
\hline $\mathrm{H}$ & 5.6327610000 & -2.4380260000 & 1.4893460000 \\
\hline $\mathrm{H}$ & 6.4473600000 & -2.7736870000 & -0.0480980000 \\
\hline $\mathrm{H}$ & 0.5195240000 & 3.0049800000 & -0.2945620000 \\
\hline $\mathrm{H}$ & 1.4366870000 & 2.7720900000 & -1.7889370000 \\
\hline $\mathrm{H}$ & 2.7037020000 & -3.1613220000 & 1.7062410000 \\
\hline
\end{tabular}




$\begin{array}{lrrr}\mathrm{H} & 1.8730710000 & -3.7098120000 & 0.2389130000 \\ \mathrm{H} & 5.4917680000 & -0.2696560000 & 2.1426020000 \\ \mathrm{H} & 4.7710180000 & 1.3554760000 & -2.4486020000 \\ \mathrm{H} & 1.9766970000 & -1.9524550000 & -1.7755130000 \\ \mathrm{H} & 1.8037300000 & 1.5177880000 & 1.7360730000 \\ \mathrm{H} & -2.4583710000 & 4.8351010000 & -2.7004810000 \\ \mathrm{H} & -1.0098350000 & 4.4829010000 & -3.6769830000 \\ \mathrm{H} & -0.8360210000 & 5.1373440000 & -2.0289940000 \\ \mathrm{H} & -0.7779020000 & 2.4259150000 & -2.5903920000 \\ \mathrm{H} & -2.4193390000 & -5.7140070000 & 0.1038000000 \\ \mathrm{H} & -1.8753510000 & -5.1558060000 & 1.7123740000 \\ \mathrm{H} & -0.6999750000 & -5.3526810000 & 0.3970200000 \\ \mathrm{H} & -2.8468660000 & -3.4966290000 & -0.2909060000 \\ \mathrm{H} & -7.5514280000 & -3.8889240000 & -3.1941900000 \\ \mathrm{H} & -7.0888450000 & -4.9172860000 & -1.8121720000 \\ \mathrm{H} & -5.8482230000 & -4.3442610000 & -2.9400300000 \\ \mathrm{H} & -5.5351370000 & 4.9331860000 & 2.5575300000 \\ \mathrm{H} & -3.9246860000 & 5.2731360000 & 1.8675590000 \\ \mathrm{H} & -4.0853250000 & 4.4061760000 & 3.4202070000 \\ \mathrm{H} & -7.3588450000 & -2.2780720000 & -1.4503570000 \\ \mathrm{H} & -3.9792250000 & 3.2819520000 & 0.8094600000 \\ \mathrm{H} & -1.5320670000 & 0.6304140000 & 3.1843750000 \\ \mathrm{H} & -0.6198950000 & -0.8840620000 & 2.9812490000 \\ \mathrm{H} & -2.7051130000 & -0.6625680000 & -3.4661680000 \\ \mathrm{H} & -1.9932210000 & 0.9590800000 & -3.5996880000 \\ \mathrm{H} & -5.2031610000 & -1.1295770000 & 3.8793610000 \\ \mathrm{H} & -4.4696390000 & 0.4883300000 & 3.8814160000 \\ \mathrm{H} & -5.7412260000 & -0.8531520000 & -2.8912540000 \\ \mathrm{H} & -6.5606980000 & 0.7002110000 & -2.5952510000 \\ \mathrm{H} & -2.0203200000 & -1.9232550000 & -1.7061430000 \\ \mathrm{H} & -1.8328910000 & 1.9350880000 & 1.3311270000 \\ \mathrm{H} & -5.5123670000 & 2.0602970000 & -0.5034430000 \\ \mathrm{H} & -4.8357400000 & -2.4023860000 & 1.5137070000\end{array}$




\section{References:}

1. Ngola, S. M.; Kearney, P. C.; Mecozzi, S.; Russell, K.; Dougherty, D. A., A Selective Receptor for Arginine Derivatives in Aqueous Media. Energetic Consequences of Salt Bridges That Are Highly Exposed to Water. J. Am. Chem. Soc. 1999, 121 (6), 1192-1201.

2. Hynes, J. B., Hydroxylamine derivatives as potential antimalarial agents. 1. Hydroxamic acids. $J$. Med. Chem. 1970, 13 (6), 1235-1237.

3. Staab, H. A.; Reimann-Haas, R.; Ulrich, P.; Krieger, C., Elektron-Donor-AcceptorVerbindungen, XXX. Elektron-Donor-Acceptor-[2.2]Paracyclophane mit N,N,N',N'-Tetramethyl-pphenylendiamin (TMPD) als Donor-Einheit. Chem. Ber. 1983, 116 (8), 2808-2826.

4. $\quad \mathrm{Xu}$, J.; Wang, W.-L.; Lin, T.; Sun, Z.; Lai, Y.-H., Molecular assembly of dithiaparacyclophanes mediated by non-covalent $\mathrm{X} \cdots \mathrm{X}, \mathrm{X} \cdots \mathrm{Y}$ and $\mathrm{C}-\mathrm{H} \cdots \mathrm{X}(\mathrm{X}, \mathrm{Y}=\mathrm{Br}, \mathrm{S}, \mathrm{N})$ interactions. Supramol. Chem. 2008, 20 (8), 723-730.

5. Martin, R. B., Comparisons of Indefinite Self-Association Models. Chem. Rev. 1996, 96 (8), 3043-3064.

6. Seo, M.; Kim, J. H.; Kim, J.; Park, N.; Park, J.; Kim, S. Y., Self-Association of Bis-Dendritic Organogelators: The Effect of Dendritic Architecture on Multivalent Cooperative Interactions. Chem. Eur. J. 2010, 16 (8), 2427-2441.

7. Smulders, M. M. J.; Nieuwenhuizen, M. M. L.; de Greef, T. F. A.; van der Schoot, P.; Schenning, A. P. H. J.; Meijer, E. W., How to Distinguish Isodesmic from Cooperative Supramolecular Polymerisation. Chem. Eur. J. 2010, 16 (1), 362-367.

8. O'boyle, N. M.; Tenderholt, A. L.; Langner, K. M., cclib: A library for package-independent computational chemistry algorithms. J. Comput. Chem. 2008, 29 (5), 839-845.

9. Sheldrick, G., SHELXT - Integrated space-group and crystal-structure determination. Acta Crystallographica Section A 2015, 71 (1), 3-8.

10. M. J. Frisch, G. W. T., H. B. Schlegel, G. E. Scuseria, M. A. Robb, J. R. Cheeseman, G. Scalmani, V. Barone, G. A. Petersson, H. Nakatsuji, X. Li, M. Caricato, A. Marenich, J. Bloino, B. G. Janesko, R. Gomperts, B. Mennucci, H. P. Hratchian, J. V. Ortiz, A. F. Izmaylov, J. L. Sonnenberg, D. Williams-Young, F. Ding, F. Lipparini, F. Egidi, J. Goings, B. Peng, A. Petrone, T. Henderson, D. Ranasinghe, V. G. Zakrzewski, J. Gao, N. Rega, G. Zheng, W. Liang, M. Hada, M. Ehara, K. Toyota, R. Fukuda, J. Hasegawa, M. Ishida, T. Nakajima, Y. Honda, O. Kitao, H. Nakai, T. Vreven, K. Throssell, J. A. Montgomery, Jr., J. E. Peralta, F. Ogliaro, M. Bearpark, J. J. Heyd, E. Brothers, K. N. Kudin, V. N. Staroverov, T. Keith, R. Kobayashi, J. Normand, K. Raghavachari, A. Rendell, J. C. Burant, S. S. Iyengar, J. Tomasi, M. Cossi, J. M. Millam, M. Klene, C. Adamo, R. Cammi, J. W. Ochterski, R. L. Martin, K. Morokuma, O. Farkas, J. B. Foresman, and D. J. Fox, Gaussian 09, Revision D. 01, Gaussian Inc.: Wallingford, CT 2009.

11. Bachrach, S. M., DFT Study of [2.2]-, [3.3]-, and [4.4]Paracyclophanes: Strain Energy, Conformations, and Rotational Barriers. J. Phys. Chem. A 2011, 115 (11), 2396-2401.

12. Legault, C., Legault, CY CYLview, 1.0 b; Université de Sherbrooke: Québec, Montreal, Canada, 2009. cylview.org/, 2020.

13. Chemcraft - graphical software for visualization of quantum chemistry computations. https://www.chemcraftprog.com, 2020. 